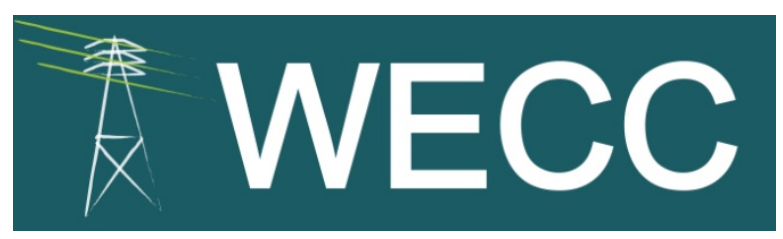

\begin{tabular}{|l|l|}
\hline Document name & $\begin{array}{l}\text { WECC Variable Generation Planning } \\
\text { Reference Book }\end{array}$ \\
\hline Category & $\begin{array}{l}\text { ( ) Regional Reliability Standard } \\
\text { ( ) Regional Criteria } \\
\text { ( ) Policy } \\
\text { ( ) Guideline } \\
\text { (x) Report or other } \\
\text { ( ) Charter }\end{array}$ \\
\hline Document date & May 14, 2013 \\
\hline Adopted/approved by & Variable Generation Subcommittee \\
\hline Date adopted/approved & May 14, 2013 \\
\hline $\begin{array}{l}\text { Custodian (entity } \\
\text { responsible for } \\
\text { maintenance and } \\
\text { upkeep) }\end{array}$ & $\begin{array}{l}\text { Physical location: } \\
\text { Stored/filed }\end{array}$ \\
\hline Previous name/number & $\begin{array}{l}\text { (if any) } \\
\text { ( ) in effect } \\
\text { ( ) usable, minor formatting/editing required } \\
\text { ( ) modification needed } \\
\text { ( ) superseded by } \\
\text { ( ) other } \\
\text { ( ) obsolete/archived) }\end{array}$ \\
\hline Status & \\
\hline
\end{tabular}




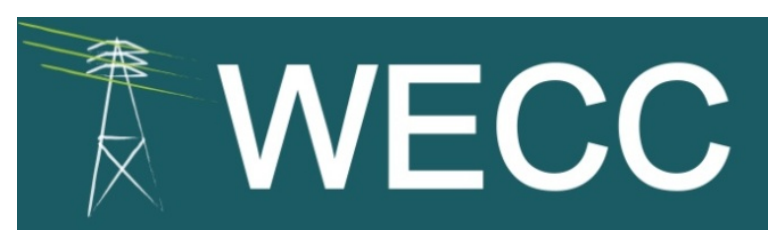

\title{
WECC Variable Generation Planning Reference Book
}

\begin{abstract}
A Guidebook for Including Variable Generation in the Planning Process
\end{abstract}

Volume 1: Main Document

Version 1

May 14, 2013 


\section{Project Lead}

Dr. Yuri V. Makarov, Chief Scientist - Power Systems, Pacific Northwest National Laboratory (PNNL)

\section{Western Electricity Coordinating Council (WECC) Project Manager}

Matthew Hunsaker, Renewable Integration Manager

\section{Contributors}

Art Diaz-Gonzalez, Supervisory Power System Dispatcher, Western Area Power Administration (WAPA)

Ross T. Guttromson, PE, MBA, Manager Energy Storage, Sandia National Laboratory

Dr. Pengwei Du, Research Engineer, PNNL

Dr. Pavel V. Etingov, Senior Research Engineer, PNNL

Dr. Hassan Ghoudjehbaklou, Senior Transmission Planner, San Diego Gas \& Electric (SDG\&E)

Dr. Jian Ma, PE, PMP, Research Engineer, PNNL

David Tovar, Principal Electrical Systems Engineer, El Paso Electric Company

Dr. Vilayanur V. Viswanathan, PNNL

Dr. Bharat Vyakaranam, PNNL

\section{WECC Member Reviewers}

Variable Generation Subcommittee Members

Antonio Alvarez, Manager IRP, Pacific Gas and Electric (PG\&E)

Steve Enyeart, Customer Service Engineering, Bonneville Power Administration (BPA)

Yi Zhang, California Independent System Operator (ISO)

\section{PNNL Peer Reviewers and Content Advisors}

Dr. Krishnappa Subbarao, Senior Research Engineer

Dr. Pavel V. Etingov, Senior Research Engineer

Dr. Landis Kannberg, Manager, Energy Storage and Renewables Integration

Dr. Ning Lu, Senior Research Engineer, previous PNNL employee

Dr. Ronald Melton, Senior Staff Engineer

\section{Acknowledgements}

U.S. Department of Energy (DOE) Office of Electricity Delivery and Energy Reliability

WECC Variable Generation Subcommittee (VGS) Members

Ravi Aggarwal, BPA

Gil Bindewald, DOE

Linda Brown, SDG\&E

Landis Kannberg, Lead Manager - Energy Storage, PNNL

Carl Imhoff, Business Line Manager, PNNL

Dale King, Product Line Manager, PNNL

Dmitry Kosterev, BPA

Mariam Mirzadeh, SDG\&E

Bradley Nickell, Director of Transmission Planning, WECC

Robert Sparks, Manager, Grid Planning South, California ISO 


\section{Table of Contents}

Figures vii

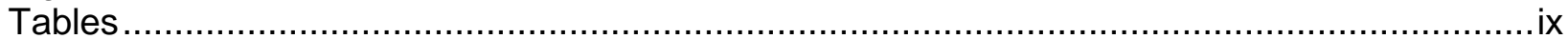

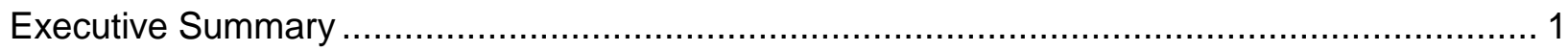

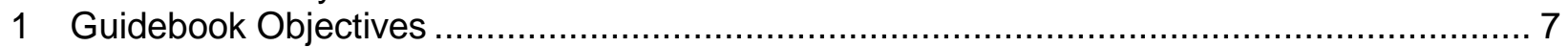

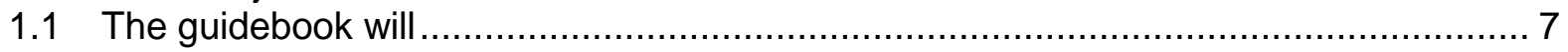

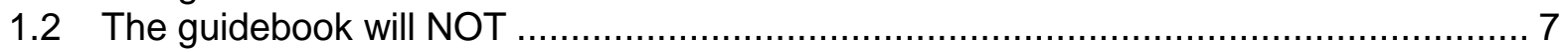

2 Variable Renewable Generators and Their Impacts on Transmission ............................ 9

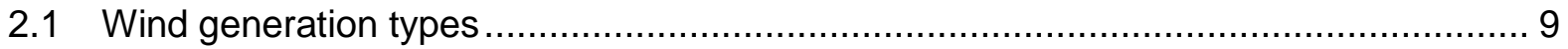

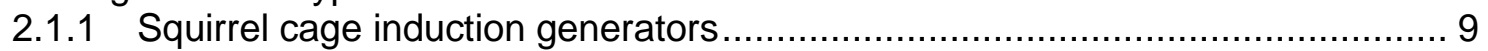

2.1.2 Doubly fed (wound rotor) induction generator ....................................... 9

2.1.3 Direct-drive synchronous generator ................................................ 9

2.2 Local distribution level impacts and interconnection issues ................................10

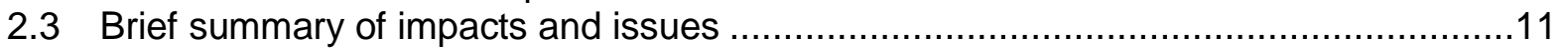

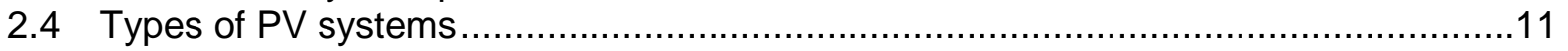

2.5 Impacts of solar photovoltaic generators on power system stability and voltage

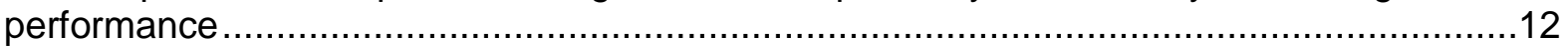

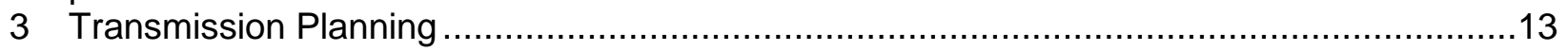

3.1 Probabilistic and multi-variant transmission planning .........................................

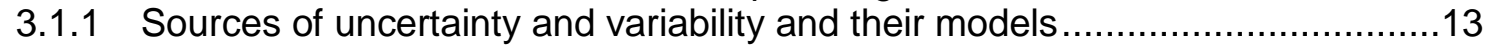

3.1.2 Deterministic planning versus a probabilistic planning .............................14

3.1.3 Simulation techniques to capture the variability of VG ...............................15

3.1.4 Probabilistic reliability criteria and analyses ............................................

3.2 Evaluation and provision of transmission capacity for VG integration ......................22

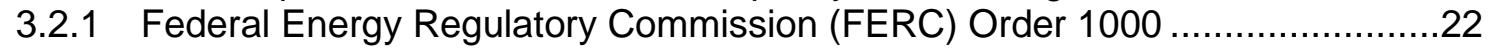

3.3 Better utilization of transmission assets .........................................................23

3.3.1 More accurate and adaptive characterization of the system security conditions 23

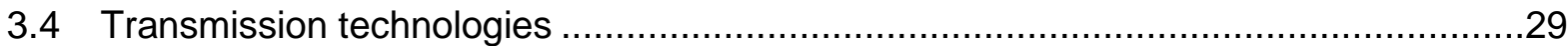

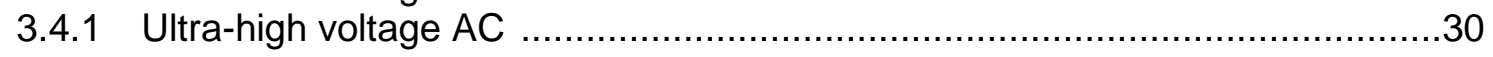

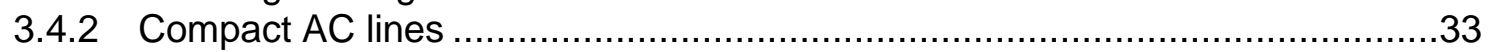

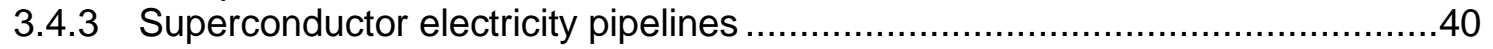

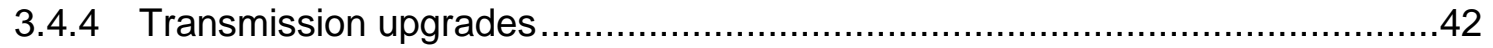

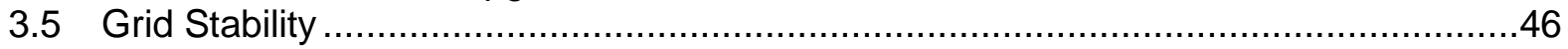

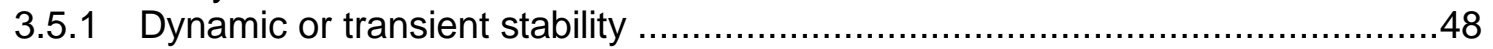

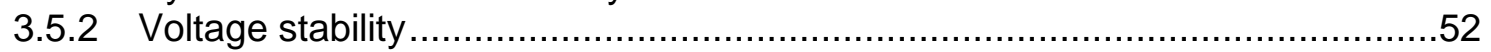

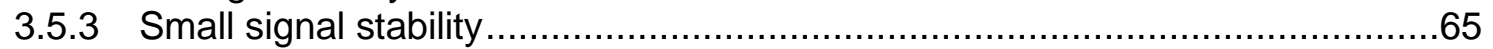

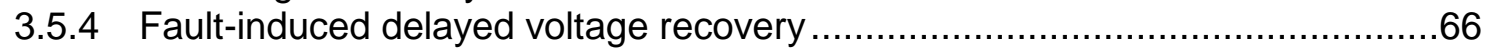

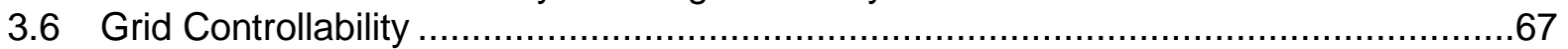

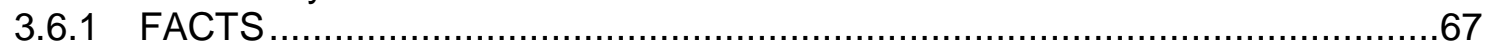

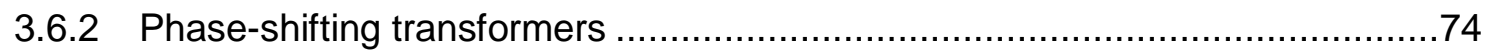

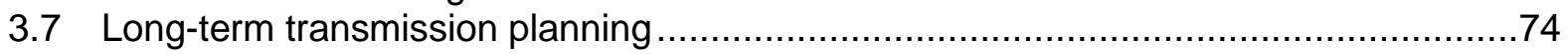

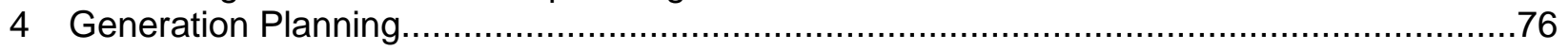

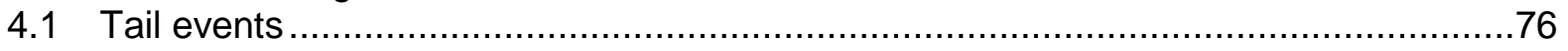

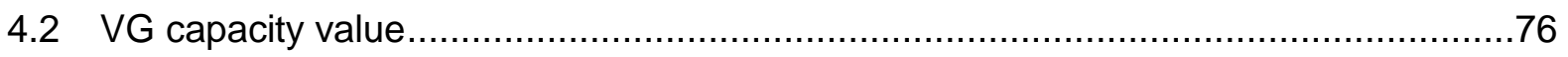

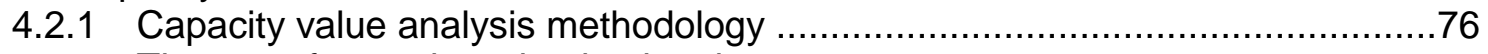

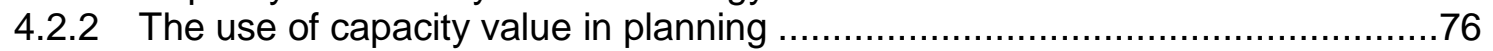


4.2.3 Wind generation capacity value (task force on the capacity value of wind

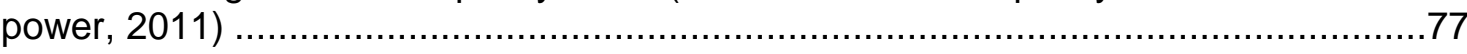

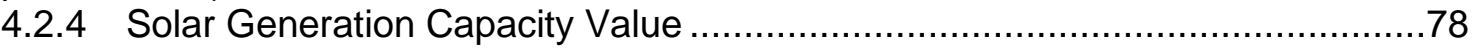

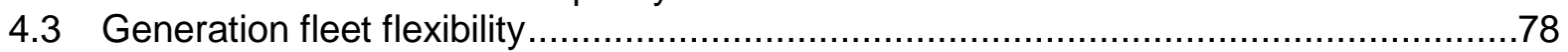

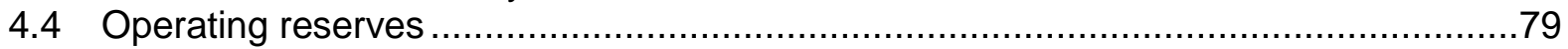

4.4.1 WECC Standard BAL-STD-002-1 - Operating Reserves ...........................81

4.4.2 Suggested changes to WECC operating reserves standard ......................... 82

4.4 .3 Impact of variable generation on reserves............................................. 82

4.4.4 Impact of new control performance standards on reserves .......................... 85

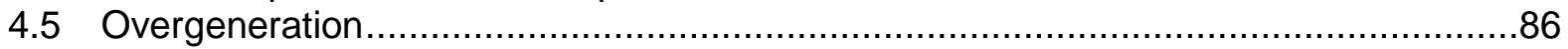

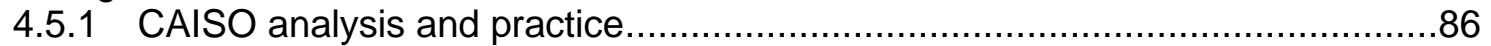

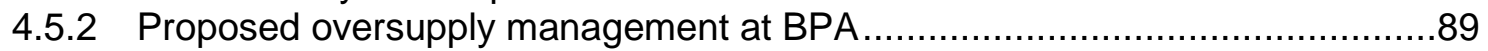

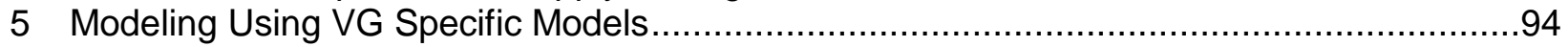

5.1 Models developed by Modeling and Validation Work Group and REMTF ................94

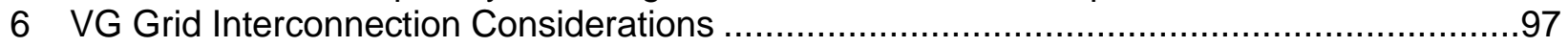

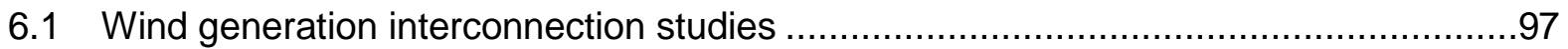

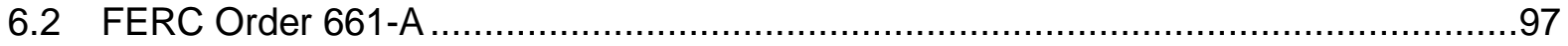

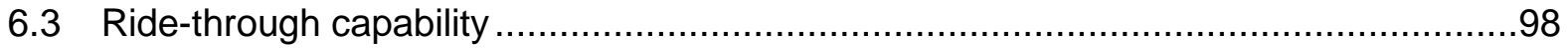

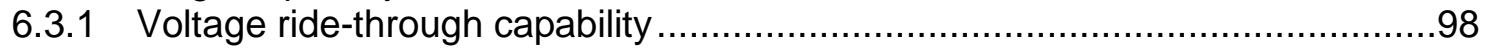

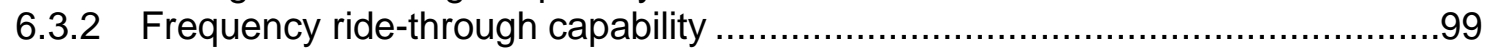

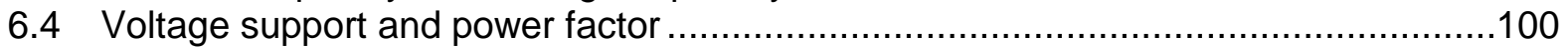

6.4.1 Discussion on the role of frequency response ...................................... 101

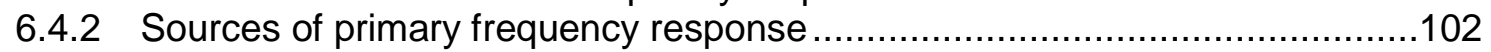

6.4.3 Calculation of frequency response from simulations or experimental data ....103

6.4.4 Existing and proposed frequency response standards .............................104

6.5 Western Renewable Energy Zones .............................................................

6.5.1 The Path Toward Western Renewable Energy Zones................................105

7 Extreme Events, Cascading Failures, and Blackouts.............................................109

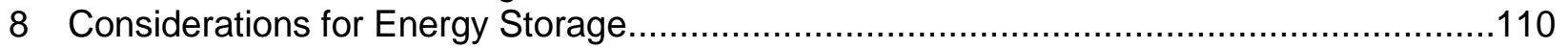

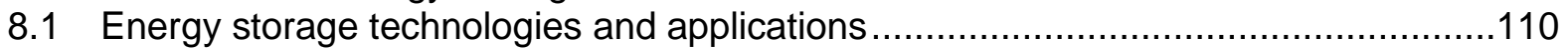

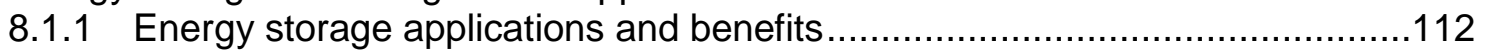

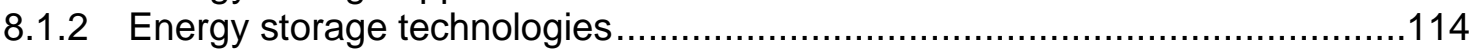

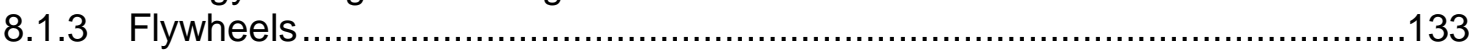

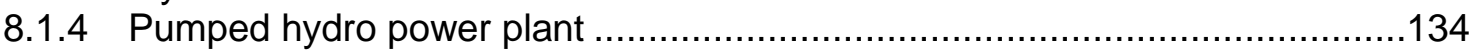

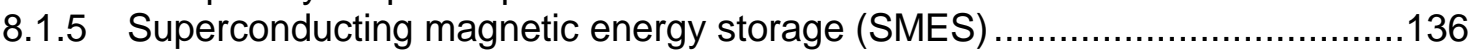

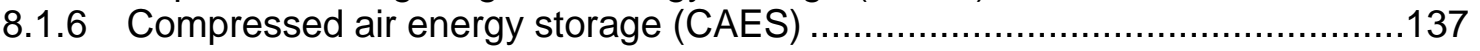

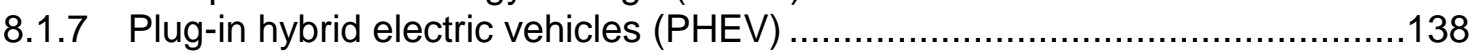

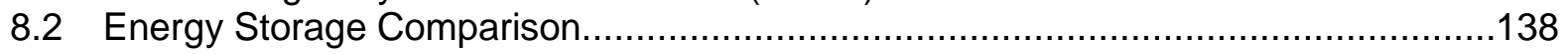

9 Considerations for Demand Response (NERC 2010a) ......................................141

9.1 Capability/feasibility of providing system reliability functions (NERC 2010a) ............143

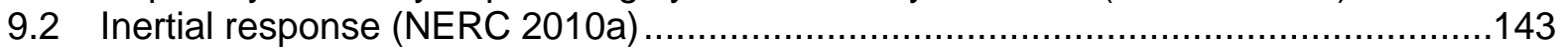

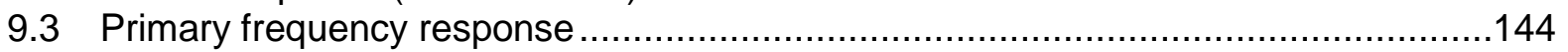

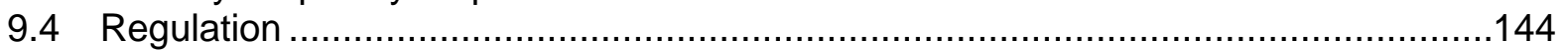

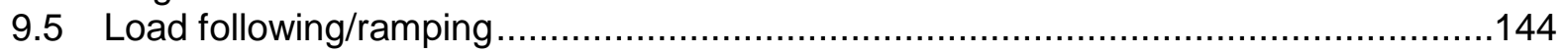

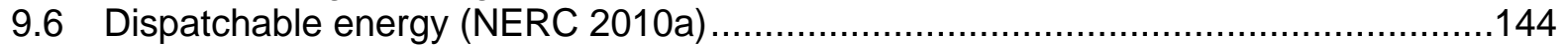

9.7 Contingency spinning reserve service (NERC 2010a) .......................................

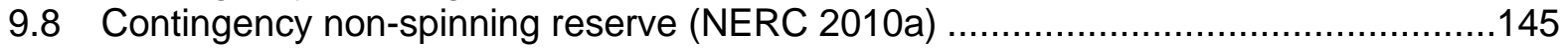

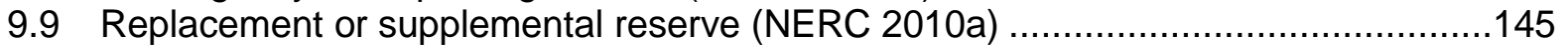

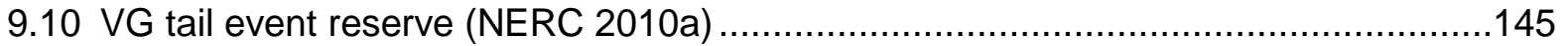

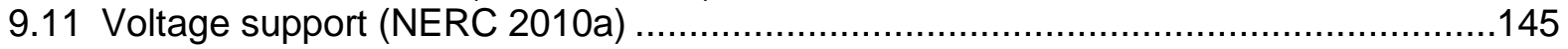

9.12 Wide-scale deployment and associated risks (NERC 2010a) ...............................146 
9.13 Distribution interconnection - technical concerns (Kroposki 2009) ........................147

9.13.1 Short-circuit current coordination (Kroposki 2009) ..................................147

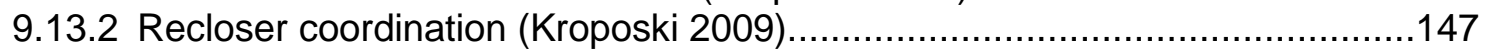

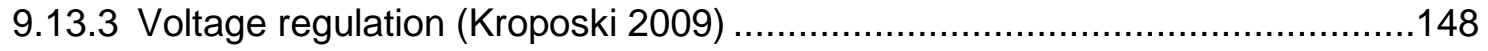

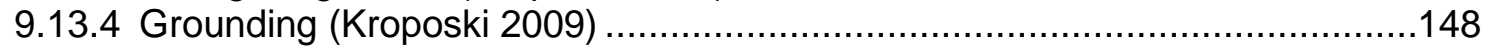

9.13.5 Power quality issues (Kroposki 2009) ..................................................

9.13.6 Intentional and unintentional islanding (Kroposki 2009)...........................149

10 Other Considerations Influencing Grid Planning ..................................................151

10.1 Consolidation and cooperation among BAs (Makarov et al. 2010a) ........................151

10.1.1 Challenges facing individual BAs at high VG penetration levels ..................151

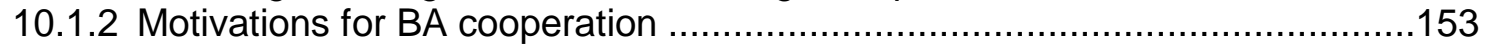

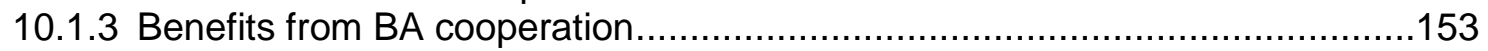

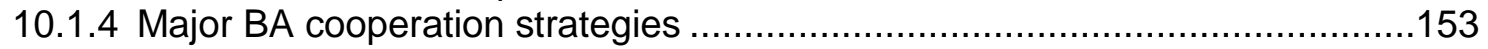

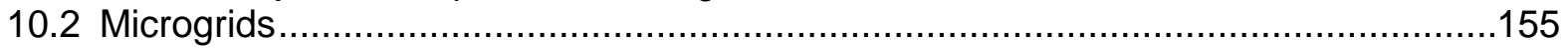

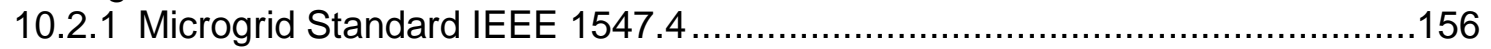

10.2.2 Microgrid Planning and Design (Butt 2011) .........................................

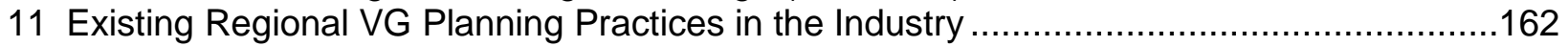

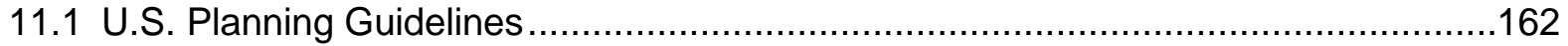

11.1.1 California ISO Interconnection Standards Review Initiative .......................162

11.1.2 Bonneville Power Administration (BPA)..............................................165

11.1.3 Midwest ISO Multi-Value-Based Planning Process ...............................166

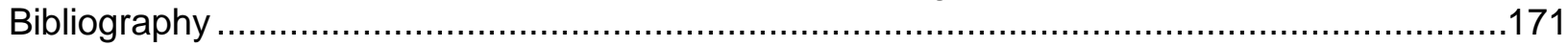




\section{Figures}

Figure 1. An example of an estimated probability density function. 16 Figure 2. Some possible risk analysis methods depending on severity and frequency of events .

Figure 3. Transmission capability in megawatts of $\mathrm{AC}$ lines with 50 percent compensation. .....31

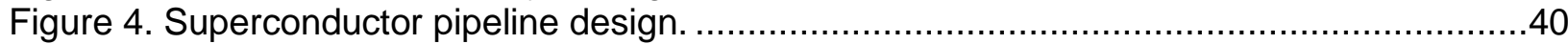

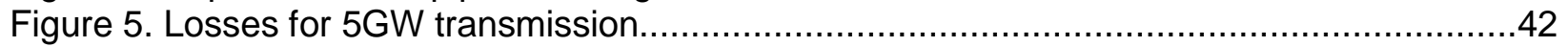

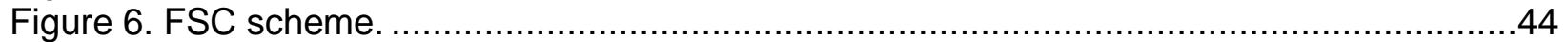

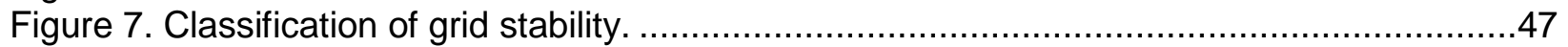

Figure 8. Voltage transient performance parameters....................................................51

Figure 9. Determination of the dynamic reactive power resource requirement. .....................54

Figure 10. Determination of the dynamic reactive power resource requirement. .....................55

Figure 11. Predictor-corrector process. ....................................................................60

Figure 12. Proposed WECC voltage ride-through requirements for all generators. .................64

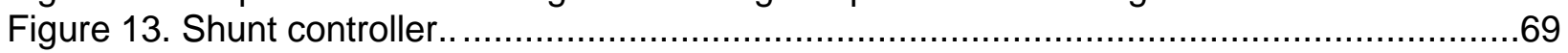

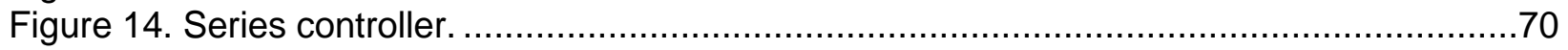

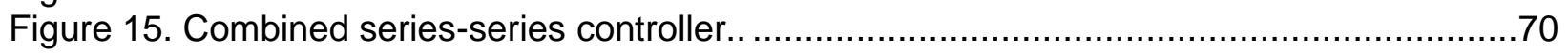

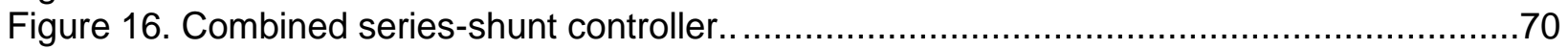

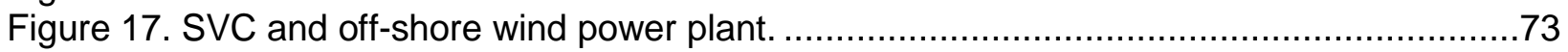

Figure 18. Example of Grid Code requirements: fault ride-through capability........................73

Figure 19. Reserve deployment as defined by NERC. ............................................... 80

Figure 20. Simulated upward and downward 5-minute load following capability for.................87

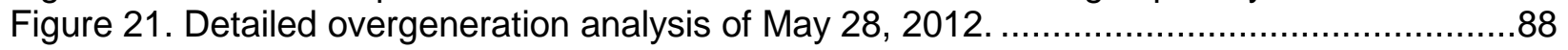

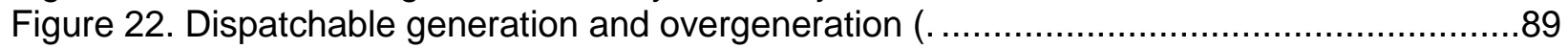

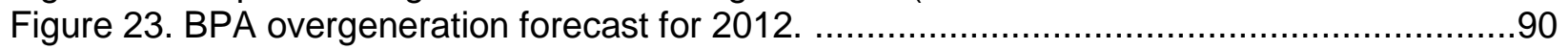

Figure 24. Four major WTG topologies and their generic models.................................95

Figure 25. Simulated Western Interconnection system frequency over the first 19 seconds

following the sudden loss of the $2,800 \mathrm{mw}$ of generation for the high reserves case..............103

Figure 26. Steps being taken to move from identification of renewable resources to a WREZ.106

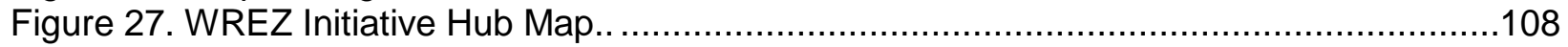

Figure 28. Output energy densities of different energy storage technologies. .....................110

Figure 29. Ratings of various energy storage technologies. .........................................111

Figure 30. Schematic of the ultrabattery electrode configuration. ....................................117

Figure 31. Cycle life of the ultrabattery compared with conventional lead-acid and Ni-MH HEV batteries charge (62s) and discharge (60s) at $3^{\circ} \mathrm{C}$ rate, with $10 \mathrm{~s}$ rest after charge and discharge.

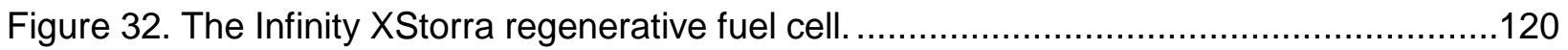

Figure 33. Schematic of the working principle of a NaS cell. ........................................122

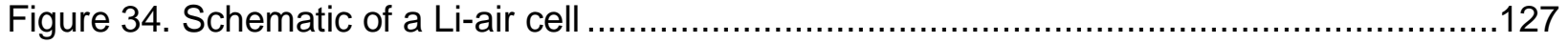

Figure 35. (left) 1-MW/250-kWh mobile Altairnano Li-ion energy storage system deployed at

PJM; and (right) A123 Systems' 12-MW Li-ion Hybrid Ancillary Power Units (Hybrid-APUs ${ }^{\mathrm{TM}}$ ),

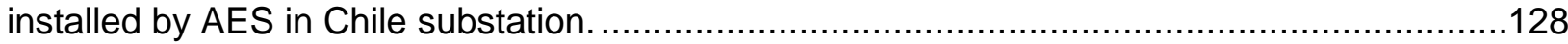

Figure 36. Prudent Energy 5-kW/30-kWh Vanadium Redox Flow Battery System installed at

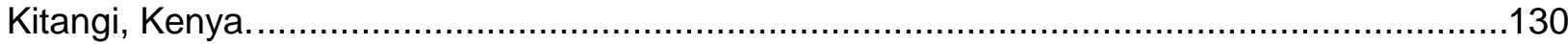

Figure 37. (left) 5-kW/20-kWh RedFlow Power community energy storage systems. (right)

Premium Power's 0.5-MW/2.8-MWh TransFlow 2000 Transportable zinc-bromine energy

storage system.

Figure 38. NERC and NAESB characterization of demand response. 


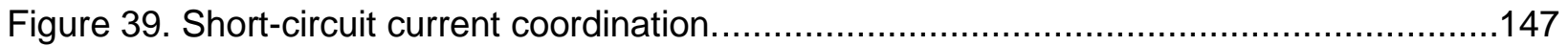

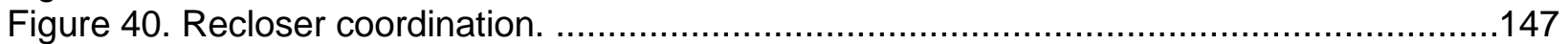

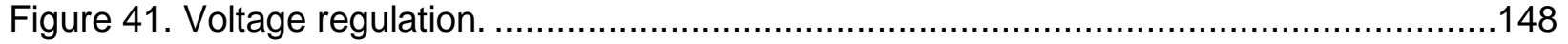

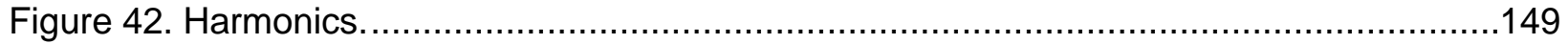

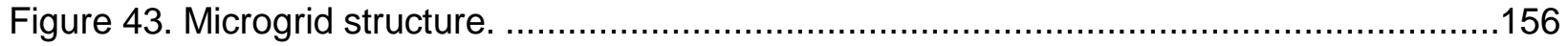

Figure 44. Examples of distributed resources island systems. ......................................157

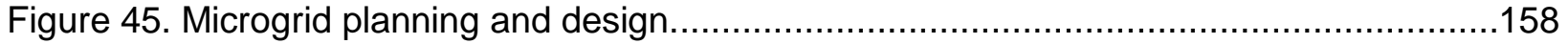

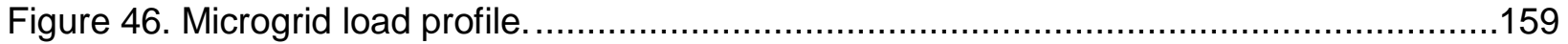

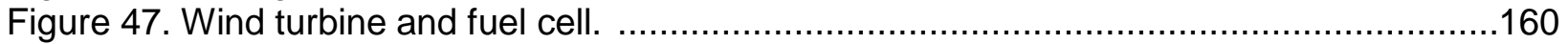




\section{Tables}

Table 1. Comparison of DC superconductor cable with AC transmission line........................41

Table 2. WECC disturbance-performance table of allowable effects on other systems. ...........51

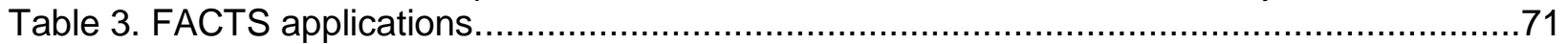

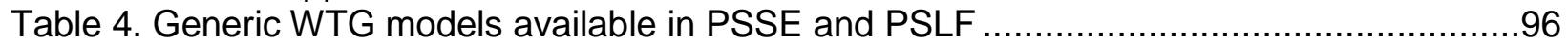

Table 5. NERC PRC-024-1 voltage ride-through performance requirement $\quad$..........................99

Table 6. WECC generator ONF performance requirement ...........................................100

Table 7. Storage applications and benefits...............................................................

Table 8. The Application matric- A Logical Analytical Framework. ..................................114

Table 9. Properties of various metal-air chemistries (* - actual Open Circuit Voltage (OCV)..125

Table 10. Comparison of the various Premium Power modules. ........................................133

Table 11 Summary of capital cost diversity for flywheel systems ...................................136

Table 12. Comparison of various energy storage technologies. .......................................139

Table 13. The scope of the MVP portfolio analysis. ............................................... 


\section{Executive Summary}

Renewable variable generation (VG) will penetrate the power grid at a high level, as required by Renewable Portfolio Standards (RPS) in different states. RPS in 24 states established the goal of reaching between 10 to 40 percent of renewable energy integrated into the grid from 2015 to 2030. In 2008, the U.S. Department of Energy (DOE) modeled an energy scenario in which wind would provide 20 percent of U.S. electricity by 2030 (USE Supply, 2008). The following is an excerpt from that report:

In the Western Interconnection, California has a goal of reaching 33 percent renewable generation by 2020 , including massive developments of wind and solar power. In the Pacific Northwest, the total wind power capacity is expected to reach 6,000 MW (approximately 14 percent of peak demand) by the end of 2011 (Northwest Wind Integration Forum 2007). In the Bonneville Power Administration (BPA) area, wind generation capacity will be $4,000 \mathrm{MW}$ by the end of 2012 , and will exceed $6,000 \mathrm{MW}$ by the end of 2013 (BPA 2010b).

The power grid planning process is experiencing major new challenges and facing many changes because of the increasing penetration of renewable variable generation. The ongoing process of renewables integration is concurrent with:

- unintended effects of deregulation and markets;

- new forms of cooperation and coordination between Balancing Authorities (BAs);

- uncertainty over future generation additions; and

- new elements added to the grid (such as energy storage, microgrids, and demand-side controls).

Generation is becoming more uncertain and less predictable because of uncertainties with future generation additions, the variability of renewable generation, and increasingly distributed natural and random market forces. Transmission impacts such as congestion, system stability and reliability are becoming more random. These impacts are less related to the economic dispatch operations, and the original design of the structure and elements of the transmission grids. The behavior of the entire system is more complex; it's more interrelated, more stressed, and much less predictable.

Topics traditionally addressed by grid planning (such as reliability, stability, performance, economics, and modeling) are changing significantly and require new solutions. For instance, deterministic methods traditionally used by grid planning engineers are becoming increasingly inadequate for addressing the increasing levels of variability and uncertainty in the modern power systems. New topics arise that were not historically considered by grid planning. For example, system inertia and frequency response characteristics can be affected with the increasing penetration of renewables and, because of that, grid planning requires certain considerations and decisions to mitigate these impacts.

Utilities and transmission system operators, both in the U.S. and abroad, have accumulatedsignificant experience with handling large quantities of renewable generation. Multiple issues related to grid planning, as well as many new solutions, have been addressed or discussed by the industry and the research community. But unlike system operation issues, and despite a significant information exchange and multiple sources of information, little effort has been made so far to consolidate the related planning challenges in one document. 
This document aims to gather the planning challenges articulated by engineers, regulators, reliability coordinators, equipment manufacturers, project developers, and researchers. In addition, this document reports on a variety of approaches to address these challenges, including new planning practices and new technologies that are increasingly penetrating the system; or those that are in an experimental mode.

This planning guide is the Western Electricity Coordination Council's (WECC) effort to put together multiple sources of information, and to provide:

- a clear, systemic, comprehensive outline of existing and anticipated problems;

- $\quad$ the problems' impacts on the system;

- current and proposed solutions by the industry and research community;

- planning practices;

- new technologies, equipment, and standards; and

- $\quad$ expected future trends.

This living, and periodically updated, document could help WECC, as well as practicing and younger engineers, to become better familiar with a large variety of information related to the integration of variable resources into the WECC system. It complements the need for timeconsuming information gathering and learning processes from more experienced engineers or from related literature. Of course, this guide is not a substitute for additional learning to obtain in-depth knowledge regarding specific areas of grid planning: its details, familiarity with specific areas in the system and regional practices, hands-on experience with the system models, simulation tools, and other aspects.

For more experienced practicing engineers, this guide offers a broader look at the planning process, by gathering facts from the other power systems, including the European system. It also outlines potential future challenges and new opportunities for regional planning processes.

This guide is not intended to be a "how to" manual. It only provides references, reviews and explanations regarding a variety of existing planning problems and approaches. This guide limits its content to WECC Variable Generation Subcommittee (VGS) Planning Work Group (PWG) Charter, and it is written with a firm intention to coordinate all planning guides and standards with WECC. This guide DOES NOT contain any required, statutory, or obligatory information; it DOES NOT duplicate existing planning standards or contradict existing planning standards and business practices. It DOES NOT cover operating or market functions; however, it is written with an understanding that, in the existing situation, system planning must be done in a context of a connection with system operating and market functions. Any interpretation of the information provided in this document should not be considered a recommendation by the VGS, WECC, or WECC members to adopt any of the practices, methodologies, or processes presented.

The effort behind this guide was led and extensively discussed by the WECC VGS PWG members, and by WECC engineers representing El Paso Electric Company, San Diego Gas and Electric (SDG\&E), and Western Area Power Administration (WAPA). All contributed significantly to this guide's content and provided advice. PNNL took the lead in preparation of the document, collecting input from other organizations, developing individual sections, compiling the volumes of information, and formatting and editing the document.Engineers from BPA, California Independent System Operator (CAISO aka California ISO), and Pacific Gas and 
Electric Company (PG\&E) reviewed the guide's content after its draft completion. Leading researchers at PNNL also provided internal reviews.

WECC's and PNNL's work was sponsored by the Office of Electricity Delivery and Energy Reliability (OE) at the DOE.

This guide contains multiple, direct quotations, and reused figures and tables. Each provides a source. For all references that are copyright protected, the guide drafting team obtained permission from the publishers or copyright owners. The team is thankful to the Institute of Electrical and Electronics Engineers (IEEE) Publishing House, Elsevier, John Wiley \& Sons, Science, Nature, The Electrochemical Society, EPRI, and Infinity Fuel Cell for providing permissions. The team is also grateful to the authors of the cited and reused documents for the insightful and informative materials that were used in this guide.

This guide consists of the executive summary, glossary, 11 main chapters, appendices, and the list of references exceeding 500 sources in a two-volume set. As the sources, North American Reliability Corporation (NERC), Federal Energy Regulatory Commission (FERC), IEEE, WECC, California Energy Commission (CEC), California ISO, BPA, PG\&E, South California Edison (SCE), SDG\&E, Midwest ISO, Electric Reliability Council of Texas (ERCOT), IEEE journal and conference presentations, multiple presentations, white papers, reports, and European resources were used.

Chapter 1 summarizes the objectives and formulates the main principles used by the guide's writing and reviewing teams.

Chapter 2 provides a technical introduction and briefly describes three, existing, main types of wind generators. It also provides a summary of their impacts on the bulk electric power system, which are important from the planning perspective, in terms of: congestion, transmission utilization rights, transmission rates and construction cost recovery, frequency support, stability, voltage support, reserve requirements, and impacts on conventional generation. Local impacts that are seen in the vicinity of wind farms include voltage support and stability issues, fault currents, feeders, protection system, and power quality. These impacts are induced by uncertainty and variability of wind and solar generators' power output, their technical characteristics, and by their interconnection to the grid and operation. The rest of the report gives more details and information.

Chapter 3 outlines information related to transmission planning practices that are influenced by an increase of variable generation in the system. This section combines information on the existing state-of-the-art grid planning technologies and research in the area.

Chapter 3 also outlines the needs, opportunities, existing approaches, and some of the research in the area of probabilistic planning. The need for probabilistic approaches is dictated by the increasing variability and uncertainty in modern power grids. The variability component appears whenever an assumption is made that the system parameters are constant — whereas they are changing over time, so that the difference between the assumed values and the actual values becomes evident. Uncertainty reflects our inability to accurately predict the future. Actual values slightly deviate from forecasted values. Variable generation is not the only source of variability and uncertainty in planning. Generation expansion uncertainty, uncertainty of market-driven dispatches, load uncertainty, random generator and transmission system outages, and other random factors contribute significantly to overall variability and uncertainty. It is important to build an integrated uncertainty model to correctly reflect all the impacts in their interaction. 
Chapter 4 considers some issues related to generation planning. The key topics considered are variable generation capacity value, generation fleet flexibility issues, operating reserves, and overgeneration.

Chapter $\mathbf{5}$ is devoted to VG modeling issues. The modeling problem is very important for planning purposes. This importance is explained by the need for accurate system representation in both steady state and dynamic states. Chapter 5 reflects the status of the current industry efforts to build accurate models of various types of wind turbines. It considers four, generic dynamic models developed by WECC's Wind Generator Modeling Group (WGMG). They reflect major wind turbine types and are suitable as library models available in such major software packages as Positive Sequence Load Flow (PSLF), Power System Simulator for Engineering (PSS/E), and Power World, which is used by WECC organizations.

Chapter 6 outlines the needs of wind generation interconnection studies. A successful integration of renewables requires compliance with certain conditions and principles, which are dictated by system performance, reliability and observability considerations. FERC Order 661-A establishes these requirements for large wind plants seeking to interconnect to the grid. It requires power plants to withstand voltage disturbances up to specified severity, comply with certain voltage support requirements, and transmit data to the transmission provider. Interconnection studies may address voltage ride-through capability (helping plants to withstand voltage disturbances up to specified severity), frequency ride-through capability (helping power plants to withstand frequency disturbances), voltage support and power factor (helping to support voltage levels and provide necessary reactive power).

Chapter 7 includes information on initial research on extreme events, cascading and blackouts involving variable generation resources.

Chapter 8 describes various energy storage technologies and their applications. Energy storage can play a significant role in successfully integrating variable renewable resources into the system. Energy storage can help to mitigate the impacts of variability and uncertainty on a power system, prevent overgeneration, provide ancillary services to the grid, as well as other services. Chapter 8 concentrates information on essential characteristics of energy storage important for planning, describes existing projects, and outlines the current rapid technology development.

Chapter 9 considers various aspects of demand response. Demand response can help to accommodate large amounts of renewable energy into the grid. Various possible applications of demand response are considered in Chapter 9, including balancing services, frequency response, simulated inertia, reserves, and voltage support. Integration of demand responsive controls in the system can cause certain problems and requires specific studies during the planning process. Chapter 9 describes some of these studies: protection coordination, short circuit analysis, voltage regulation, and grounding.

Chapter 10 considers some additional analyses and technologies, including the actual and virtual consolidation of BAs and microgrids. Consolidation and cooperation methods among the BAs allow exploiting the diversity effects of geographically distributed variable energy resources. Relative impacts of uncertainty and variability on the grid are decreasing with the increasing number and spatial distribution of variable generators. Chapter 10 analyzes different possible 
forms of consolidation and cooperation between BAs. Chapter 10 is also discussing integration issues related to microgrids.

Chapter 11 is a summary of planning practices in the United States and Europe. Specific attention is paid to the CAISO, BPA, and Midwest ISO planning guidelines and their development. Variable generation interconnection requirements in Germany and Spain are also outlined in Chapter 11.

\section{Appendices}

Volume 2 of the report includes 27 appendices:

Appendix 1 provides the glossary.

Appendix 2 provides the recent approaches to the probabilistic load flow problems.

Appendix 3 lists some additional useful indices.

Appendix 4 lists high-impact, low-frequency (HILF) events. These have recently become a renewed focus for risk managers. The information on HILF events and their impacts on electric power systems are also discussed in this section.

Appendix 5 provides examples of wide-area nomograms.

Appendix 6 describes elements of the theory behind transmission line ratings, types of dynamic rating methods, and potential implications of using dynamic line ratings to the power and renewable industry.

Appendix 7 presents the relative costs per MW-km of different electric power transmission technologies.

Appendix 8 presents the national and international experience in ultra-high voltage (UHV) transmission

Appendix 9.1 discusses the characteristics of voltage-sourced converter- high-voltage direct current (VSC-HVDC).

Appendix 9.2 provides additional information on the voltage, current and project configuration in HVDC.

Appendix 9.3 provides additional information on the selection of rated current in HVDC.

Appendix 9.4 lists the advantages of DC systems over AC systems for integration of renewable energy.

Appendix 9.5 lists the national and international HVDC projects.

Appendix 10 provides additional information on rewiring of existing transmission lines.

Appendix 11 lists the types of high-temperature low sag (HTLS) conductors. 
Appendix 12 describes the direct method and energy functions for transient stability analysis in power systems.

Appendix 13 shows blackouts caused by voltage instability

Appendix 14 describes the algorithm for parameter continuation predictor-corrector methods.

Appendix 15 discusses some approximation techniques available for security regions.

Appendix 16 describes impact of wind power on power system small signals stability.

Appendix 17 provides some additional information on the fault-induced delayed voltage recovery (FIDVR).

Appendix 18 describes some commonly used transmission system (FACTS) controllers.

Appendix 19 discusses the European planning standard and practices.

Appendix 20 presents international experience in wind and solar energy sources.

Appendix 21 presents the impacts of variable generation on inertia and frequency response.

Appendix 22 presents Western Renewable Energy Zones (WREZ) initiative renewable energy generating capacity tables.

Appendix 23 provides additional details on various energy storage technologies.

Appendix 24 provides additional information on the demand response.

Appendix 25 provides a summary of various BA consolidation and cooperation options.

Appendix 26 provides some additional information on generator power management requirements.

Appendix 27 provides European planning guidelines. 


\section{Guidebook Objectives}

Renewable VG will penetrate the power grid at a high level, as required by different states' Renewable Portfolio Standards (RPS). The RPSs of 24 states establish the goal of reaching between 10 and 40 percent of renewable energy integrated into the grid by 2015-2030. The U.S. Department of Energy (DOE) has modeled an energy scenario in which wind would provide 20 percent of U.S. electricity by 2030 (USE Supply, 2008). In the Western Interconnection, California has a goal of reaching 33 percent of electricity from renewable sources by 2020 , including massive developments of wind and solar power. In the Bonneville Power Administration (BPA) area, wind generation will exceed 6,000 MW by 2013 (BPA 2010b).

Variable renewable generation has a profound impact on grid planning. It requires changes to be made in existing practices, the consideration of new issues, the addition of new models, and it requires addressing new concerns. Remarkably, grid planning now has to consider everincreasing issues related to future grid operations. For instance, new generation additions should provide a sufficient set of flexibility characteristics that are needed to follow combined variations of variable generation and load in future, real-time operation. Moreover, there is a rapidly growing understanding that major changes in the grid planning philosophy are needed. For example, variable generation stresses the need for probabilistic approaches to transmission planning.

The U.S. Department of Energy funded the creation of this two-volume document. It is the result of a collective effort of the Western Electricity Coordinating Council (WECC) Variable Generation Subcommittee's (VGS) Planning Work Group (PWG) to provide a broad and systemic view of the problems related to the increasing penetration of renewables. It seeks to outline best practices, and indicate where changes are needed in the grid-planning practice. The use of specific trade names and manufacturers is for research purposes only, and does not constitute an endorsement of these products.

\subsection{The guidebook will}

- Document the best practices that exist and those that are under development;

- Explain the conditions that make various issues a higher versus lower priority:

o Explain why they are important; and

o Explain why, when and how planning engineers should address them;

- Provide references or modest suggested approaches to address them (this is not intended to be a technical, "how to" manual);

- Coordinate with WECC planning guides and standards; and

- Limit content to topics within the VGS PWG charter.

\subsection{The guidebook will NOT}

- Be required, statutory, or obligatory;

- Duplicate existing planning standards;

- Contradict existing planning standards; and

- Cover operating or market functions, however: 
o System planning must be done in context of system operations and market functions. 


\section{Variable Renewable Generators and Their Impacts on Transmission $^{1}$}

This section gives an introduction to wind turbine technologies and the impact of wind generation on the distribution and transmission system. The rest of the chapter provides more details and more information on these topics.

"In wind turbines, generating systems different from the synchronous generator used in conventional power plants are applied. The prime mover of wind turbines, i.e., the wind, cannot be controlled, and fluctuates randomly. Up to this moment, the generated power of wind turbines is completely determined by the wind speed and not controlled any further. The typical size of wind turbines is much lower than that of a conventional power plant" (Murty undated).

\subsection{Wind generation types}

This section is a brief summary of wind generation technologies.

\subsubsection{Squirrel cage induction generators}

Squirrel cage induction generators are nothing but conventional, directly grid-connected squirrel cage induction generators (Murty undated).

"The slip, and hence the rotor speed of a squirrel cage induction generator varies with the amount of power generated. These rotor speed variations are, however, very small, approximately 1 to 2 percent. Therefore, this wind turbine type is normally referred to as a constant speed or fixed speed turbine. A squirrel cage induction generator always absorbs reactive power. In most cases, this is undesirable, particularly in case of large turbines and weak grids. Reactive power consumption of the squirrel cage induction generator is nearly always partly or fully compensated by capacitors in order to achieve a power factor close to one" (Murty undated).

Squirrel cage generators "can run at two different (but constant) speeds by changing the number of pole pairs of the stator winding" (Murty undated).

\subsubsection{Doubly fed (wound rotor) induction generator}

"In the doubly fed induction generator, a back-to-back voltage source converter feeds the three phase rotor winding. In this way, the mechanical and electrical rotor frequencies are decoupled, and the electrical stator and rotor frequency can be matched, independently of the mechanical rotor speed" (Murty undated).

\subsubsection{Direct-drive synchronous generator}

"In the direct drive synchronous generator, the generator is completely decoupled from the grid by a power electronics converter" (Murty undated).

\footnotetext{
${ }^{1}$ Contributed by Yuri Makarov, PNNL.
} 


\subsection{Local distribution level impacts and interconnection issues}

Local impacts are observed "in the (electrical) vicinity of a wind turbine or wind park that can be attributed to a specific turbine or park, i.e., of which the cause can be localized. Local impacts differ for the three main wind turbine types" (Murty undated).

"Wind power locally has an impact on the following characteristics and equipment (Murty undated, Smith 2004, and Wind 2006):

- Power flows and nodal voltages;

- Impact of distributed wind on distribution feeders;

- Voltage ride-through capability;

- Fault currents;

- Protection schemes, overcurrent protective device coordination, and switchgear ratings;

- Harmonics; and

- Flicker.

"Voltage at the turbine's terminals is normally lower than the voltage of the grid to which it is connected, leading to the need for a transformer" (Murty undated).

"During normal operation, changes in $\mathrm{kW}$ generation will change the voltage level on the feeder. It is possible, but very unlikely, that gusty wind could change the kW enough so that it would cause the voltage level on the feeder to change enough to be noticeable. To be noticeable, the following conditions would be needed:

- A very weak grid, such as a 2.4-kV system, or a long distance from the substation.

- A combination of wire size and distance from substation.

- A wind turbine with high flicker characteristics" (Wind 2006).

The impact of large wind turbines on a distribution feeder includes the following:

- Reduced power flow from the substation;

- May cause back-feed into substation;

- Can cause unusual increase of voltage levels on feeders;

- The substation voltage regulators may experience reverse power flow; and

- May increase or decrease distribution losses (Wind 2006).

Coordination of feeder protection should provide the following characteristics:

- Minimize the impact of wind turbines on feeder reliability;

- Wind turbines should trip off on their own for disturbances, such as faults and over- and under-voltages;

- Wind turbines should also trip for phase imbalance and over/under frequency, and

- Wind turbines should not cause unnecessary recloser's operations (Wind 2006). 
"Switchgear is necessary to disconnect the wind turbine in case of a short circuit or in order to prevent what is called islanding, a situation in which a small part of the grid continues to operate with a local balance between generation and load, but without being connected to the main system" (Murty undated).

"During startup and generator switching, there will be inrush currents, which will cause the voltage to dip or flicker. Voltage flickers may or may not be noticeable or objectionable. This depends upon magnitude and how often it occurs. Magnitude of flicker depends on the stiffness of the line, voltage level, distance from substation, size of substation transformer, and wind turbine electrical design" (Wind 2006).

"Analytical tools for distribution system planning, design, and operation with radial distribution feeders may no longer be valid for feeders interconnected to distributed generators" (Smith 2004).

\subsection{Brief summary of impacts and issues}

System-wide, transmission-level impacts "are strongly related to the penetration level in the system as a whole" (Murty undated). As a result of its remote nature, wind generation needs longer transmission. It is competing with traditional generation for transmission. "Project financing requires transmission certainty. Transmission issues have the potential to derail wind development" (Smith 2004).

System-wide impacts and issues include:

- Congestion;

- Markets for transmission rights;

- Rate pancaking;

- Frequency ride-through capability;

- Impacts on dynamics and stability;

- Reactive power generation/voltage control

- System balancing, reserve requirements, and frequency control;

- Dispatch of the remaining conventional units, and

- Transmission cost recovery and allocation (Murty undated and Smith 2004).

"When large numbers of wind turbines are connected to a system, and they replace a substantial fraction of the output of the conventional synchronous generators, they will start to affect various aspects of the system behavior. This particularly will be the case during periods with low loads and high-wind speeds because in these situations, the relative contribution of wind power is at its maximum" (Murty undated).

\subsection{Types of PV systems}

"Solar photovoltaics (PV) are semiconductor devices that convert sunlight directly into electricity. They do so without any moving parts and without generating any noise or pollution. They must be mounted in an unshaded location; rooftops, carports and ground-mounted arrays are 
common mounting locations. The amount of energy produced by a panel depends on the several factors. These factors include the type of collector, the tilt and azimuth of the collector, the temperature, the level of sunlight and weather conditions. An inverter is required to convert the direct current (DC) to alternating current (AC) of the desired voltage compatible with building and utility power systems. The balance of the system consists of conductors/conduit, switches, disconnects and fuses. Grid-connected PV systems feed power into the facility's electrical system and do not include batteries" (Salasovich, J., and Mosey. G., 2011).

\section{Ground-mounted systems}

"On a \$/DC-Watt basis, ground-mounted PV systems are usually the lowest-cost option. Several PV panel and mounting options are available, each having different benefits for different ground conditions.

"To get the most out of the available ground area, considering whether a site layout can be improved to better incorporate a solar energy system is important. If unused structures, fences, or electrical poles can be removed, the unshaded area can be increased to incorporate more PV panels. When considering a ground-mounted system, an electrical tie in location should be identified to determine how the energy would be fed back into the grid.

"Fixed-tilt systems are installed at a specified tilt and are fixed at that tilt for the life of the system. Single-axis tracking systems have a fixed tilt on one axis, and a variable tilt on the other axis. The system is designed to follow the sun in its path through the sky. This allows the solar radiation to strike the panel at an optimum angle for a larger part of the day than can be achieved with a fixed-tilt system. A single-axis tracking system can collect nearly 30 percent more electricity per capacity than can a fixed-tilt tracking system. The drawbacks include increased operations and maintenance costs, less capacity per unit area (DC-Watt/ft2), and greater installed cost (\$/DC-Watt).

"In many cases, a roof is the best location for a PV system. Roof-mounted PV systems are usually more expensive than ground-mounted systems, but a roof is a convenient location because it is out of the way and is usually unshaded. Large areas with minimal rooftop equipment are preferred, but equipment can sometimes be worked around if necessary. If a building has a sloped roof, a typical flush-mounted crystalline silicon panel can achieve power densities on the order of $11 \mathrm{DC}$-Watt/ft2. For buildings with flat roofs, rack-mounted systems can achieve power densities on the order of $8 \mathrm{DC}-$ Watt/ft2 with a crystalline silicon panel. Typically, PV systems are installed on roofs that either are less than five-years old or have over 30 years of life left. Because no roof area is available on the sites studied no roof-mounted analysis was conducted" (Salasovich, J., and Mosey. G., 2011).

\subsection{Impacts of solar photovoltaic generators on power system stability and voltage performance}

"Solar PV (Photovoltaic) generators can be connected to a transmission system as a large-scale installation or distributed across a distribution system close to load. Solar PV generator interconnection may cause oscillation problems following faults, high-voltage problems in subtransmission and distribution systems under normal conditions, and the transient overvoltage problem in the grid following faults. These problems may cause further reliability concerns such as overload of sub-transmission and distribution lines, over-voltage generation tripping, and system-wide transient instability. Mitigation, including, reactive power capability from the generation, and additional fast acting reactive power support, may be needed to mitigate the reliability problems" (Zhang et. 2012). 


\section{Transmission Planning}

This section considers new aspects of the transmission planning process arising from the increasing penetration of variable energy resources.

\subsection{Probabilistic and multi-variant transmission planning ${ }^{2}$}

Transmission planning is essentially driven by the economics (least-cost option/prime rate of return) and reliability of the bulk electric system, based upon an acceptable level of risk. When a plan for transmission expansion is necessitated by future growth forecasts, or, as in this planning guide, the integration of variable generation, transmission system expansion study teams focus primarily on cost and reliability.

Various modeling alternatives are available for these studies, offering differing degrees of modeling detail and complexity. Deterministic planning, based on limited N-1 criterion, or single contingency failure, has long been the methodology used in considering, accepting and planning for known/unknown risk factors. However, there are shortcomings in deterministic planning, including multiple component failures or uncertain inputs, and the accurate accounting of these factors into the algorithms. Differing from deterministic criteria planning, "probabilistic planning, through quantified reliability assessment, can capture multiple component failures and recognize not only the severity of the events but also the likelihood of their occurrence" (Li and Choudhury 2007).

Probabilistic planning captures and identifies the risk level associated with deterministic criteria, through quantified reliability assessment, which is based on the limited number of components taken out (WECC 2006). Multi-variant transmission planning, by incorporating multiple variable/scenario modeling, captures potential engineering and economic benefits offered by transmission expansion and integrating variable generation. Based on these planning simulations, the effects of transmission expansion and the integration of variable generation can be observed and studied for future growth decisions. Therefore, to broaden the engineering and economic benefits of integrating variable generation into future system planning, modeling will be enhanced substantially by employing probabilistic or multi-variant transmission planning.

\subsubsection{Sources of uncertainty and variability and their models ${ }^{3}$}

Variable generation is only one of many sources of uncertainty in planning. This section outlines the most important sources. Their correct modeling is extremely important for a probabilistic planning process. The variability component appears whenever an assumption is made that the system parameters are constant, whereas they are changing over time, so that the difference between the assumed values and the actual values becomes significant. This difference is reflected by variability metrics. For instance, for planning purposes, one can assume constant values of VG for certain operating hours, but the actual generation changes significantly within the hour.

Uncertainty reflects our inability to accurately predict the future. Actual values deviate more or less from forecasted values. For example, the actual average wind generation will not be equal to its day-ahead forecasts because of forecast errors.

\footnotetext{
${ }^{2}$ Contributed by Art Diaz-Gonzalez, Western Area Power Administration (WAPA)

${ }^{3}$ Contributed by Yuri Makarov, Pacific Northwest National Laboratory (PNNL)
} 
A deterministic planning study can be based on annual total load curves, and an assumption can be made on the curves reflecting changes of variable generation. These assumptions result in a single, analyzed scenario, but there are multiple, possible scenarios to be analyzed with their associated probabilities. These scenarios may be significantly different when compared with the single deterministic scenario because of the variability and uncertainty factors. Both the variability and uncertainty factors should be incorporated into probabilistic studies.

\subsubsection{Deterministic planning versus a probabilistic planning ${ }^{4}$}

Aging transmission systems combined with the requirement to provide higher standards of system reliability at the lowest costs possible, and the integration of variable generation are challenging the electric industry globally. Deterministic ( $N-1$ ) planning models used in transmission planning have limited application in a multi-variant environment conducive to the integration of variable generation resources. Simulation methodology using random sampling and computational algorithms offer increased supportive data that is used to calculate risk factors when planning for integrating variable generation into a BA's transmission system.

The traditional approach to deal with voltage requirements is based on the analysis of "worstcase conditions" and on the use of "safety factors." This approach has been used for many years in engineering analysis and design. It can be interpreted as a deterministic way of taking into account past experience and unexpected future events. From the planning point of view, deterministic approaches have some attractive features, such as simple implementation, easy understanding, assessment, and judgment by planners, in relation to severe conditions such as network outages and system peak load. In addition, past experience with variable renewable sources such as wind or solar power is very limited.

"The limitation of the deterministic approach is that it represents past experience and future expectation by single facts or numbers, without assigning any degree of importance or likelihood to them" (Leite da Silva et al. 1990). In reliability assessments of generation and transmission composites and transmission loss evaluation, the traditional deterministic power flow analysis can yield an extremely large number of power flow cases for the purpose of capturing all the variances of the electric load and generation dispatch schedules. In these situations, the probabilistic methods that directly treat the uncertainty of the electric load and generation should be considered (Meliopoulos et al. 1990).

"An alternative and more realistic way of incorporating past experience is to identify all events that have directly or indirectly contributed to that experience and associate these with their probability of occurrence. Once the past experience is represented by statistical parameters, the next step, comprising the application of special analysis and design techniques, is carried out within this stochastic environment. This procedure forms the basis of the probabilistic approach. Therefore, past performance can be understood and future performance can be predicted in a more consistent way" (Leite da Silva et al. 1990, Meliopoulos et al. 1990).

Methodologies based on probability concepts can be extremely useful in assessing the performance of power systems (Billinton and Allan 1996). They have been successfully (but not yet widely) applied to many areas, including generation and transmission capacity planning, operating reserve assessment, and distribution systems. The proper measure of risk can only be achieved by recognizing the probabilistic nature of power system parameters.

\footnotetext{
${ }^{4}$ Contributed by Art Diaz-Gonzalez, WAPA, Jian Ma, PNNL, and Yuri Makarov, PNNL
} 


\subsubsection{Simulation techniques to capture the variability of $\mathrm{VG}^{5}$}

VG applications, specifically wind and solar, have introduced a multitude of uncertain parametric conditions not previously encountered in the electric industry. In addition, multiple interacting factors associated with a transmission network vary, such as available and/or restricted transmission capacity, versus available and/or non-productive states of variable generation, fuel availability/price, energy market influences, and weather.

Probabilistic power flow analysis is a tool that provides the probability of a system variable, such as node voltages, or power through lines, taking a value. The goal of this analysis is to forecast the risk of line overloading and congestion. From the first proposals in the 1970s (Borkowska 1974, Dopazo 1975), a great deal of literature can be found about this subject.

Probabilistic techniques have been used to take into account a multitude of sources of uncertainties in power-systems operations for a certain system-demand range (Milligan 1996).

A frequently used method of solving this problem is the Monte Carlo simulation. This technique involves repeated simulations with randomly sampled values obtained from the probability density function (PDF) of the random variable considered. Planning models using the Monte Carlo methodology increasingly have become useful in planning for integrating variable generation. They also have proven to be invaluable in risk calculation, and in subsequent transmission expansion and variable generation integration planning. Monte Carlo modeling will aid in preventing inconclusive or unsubstantiated data. It will offer expanded data that can be applied towards a practical and organized strategic plan for variable generation integration.

The probabilistic load flow (PLF) problem can be solved by Monte Carlo simulation (MCS) techniques and linearized alternating current (AC) load flow equations (Leite da Silva et al. 1984). The density function of the total system load is simulated in conjunction with the available capacity of generating units. The linearization process starts by obtaining the probability density function of the total system load by convolving all busload density functions. The utility's operating policy then is used to balance the active power for each simulated trial. This balance can be carried out for the whole power system or for each control area of the system. At the end of this simulation process, the expected values and PDF functions for all input nodal powers are known. A conventional load flow algorithm is used with the expected nodal powers, and for a given network topology, to obtain the associated state vector (i.e., expected voltages and angles). Finally, the load flow equations are linearized around this point and some probability characteristics (such as load duration curve or probability distribution function) are estimated. Figure 1 illustrates two such characteristics (Yu et al. 2009).

\footnotetext{
${ }^{5}$ Contributed by Art Diaz-Gonzalez, WAPA, and Hassan Ghoudjehbaklou, San Diego Gas \& Electric (SDG\&E).
} 


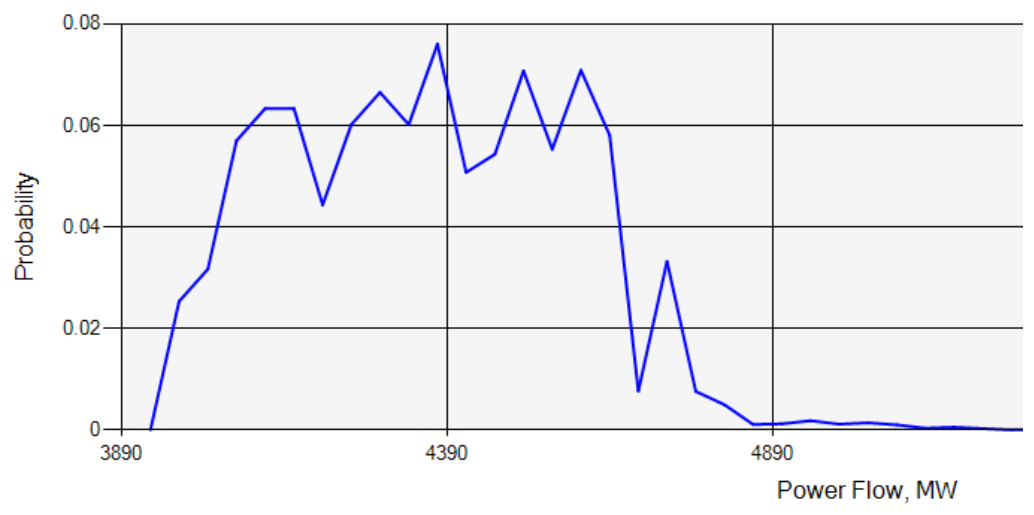

Figure 1. An example of an estimated probability density function.

One idea is to model all renewable sources as negative loads with appropriate probabilities. Then, a load duration curve similar to Figure 1 effectively would include all uncertainties regarding the renewable resources. In addition, some estimated probability density functions similar to Figure 1 for different network parameters could provide their expected values and confidence intervals. Such probabilistic tools tell us the probability of overload or voltage violation. Other Markov-based models could tell us the mean time between violations or frequency of violations (similar to mean time to failure or outage frequency). But these new ideas require more study and only might be applied on a limited scale.

"Implementation of the Monte Carlo method requires an adequate uncertainty representation, and many simulations must be considered in real systems. Sometimes this makes this approach more difficult" (Leita da Silva 2007). A different approach more suitable under certain conditions is discussed in the next section.

\subsubsection{Well-being analysis ${ }^{6}$}

"A new framework, named system well-being analysis (Billinton, R. and Khan, E. 1992; Billinton, R. and Lian G. 1994; Billinton, R. and M. Fotuhi-Firuzabad 1994; and Billinton, R. and Karki R.1999), has been built combining the deterministic perception with probability concepts. This new framework reduces the gap between deterministic and probabilistic approaches by providing the ability to measure the degree of success of any operating system state. In a wellbeing analysis, success states are further split into healthy and marginal states, using the previously mentioned engineers' perception as the criterion. Well-being analysis has been applied in the last decade to areas such as generating systems, operating reserve assessment, and composite generation and transmission systems. Chronological or sequential Monte Carlo simulation has been used for generating system well-being analysis, which considers the loss of the largest available unit in the system as the deterministic criterion. These concepts can be useful for dimensioning the reserve capacities considering renewable resources." Leite da Silva et al. 2004. The recent approaches to the probabilistic load flow problems are provided in Appendix 2.

\footnotetext{
${ }^{6}$ Contributed by Jian Ma, PNNL.
} 


\subsubsection{Probabilistic reliability criteria and analyses}

North American Electric Reliability Corporation (NERC)'s definition of "reliability" consists of two fundamental concepts: adequacy and operating reliability.

- Adequacy is the ability of the electric system to supply the aggregate electric power and energy requirements of the electricity consumers at all times, taking into account scheduled and reasonably expected unscheduled outages of system components.

- Operating reliability is the ability of the electric system to withstand sudden disturbances, such as electric short circuits or unanticipated loss of system components.

\subsubsection{This a good time to actually start using probabilistic analyses ${ }^{7}$}

The fundamental task of transmission planning is to develop or expand a transmission system as economically as possible, while maintaining an acceptable level (risk) of system reliability. Deterministic criteria, which is widely used throughout the electric industry and has long been the norm for calculating and planning system expansion, needs a reevaluation. With the integration of variable generation, planning criteria has risen exponentially. Interconnected power systems have a multitude of equipment, elements, and system dynamics that at any moment may fail and require immediate attention. No longer can transmission planners rely on the deterministic criteria. Therefore, when planning for variable generation penetration, system dynamics should be integrated into the equation. Probabilistic planning modules used for evaluating or assessing transmission system expansion, including risk-cost evaluations and modeled over specific, strategic time periods, employ multistate random variables. Capturing and integrating multicomponent failure events, including severity and probability, will undoubtedly result in a comprehensive analysis presenting a quantified risk assessment for future transmission planning. "Probabilistic planning is a fundamental and powerful tool that can provide utilities with significant economic benefits, while maintaining an acceptable system risk level" (Li 2005).

\subsubsection{Acceptable level of reliability $(A L R)^{8}$}

The acceptable level of reliability (ALR) concept is defined by NERC in NERC (2007) as follows:

"The bulk-power system ("system") will achieve an adequate level of reliability when it possesses following characteristics:

1. The system is controlled to stay within acceptable limits during normal conditions;

2. The system performs acceptably after credible contingencies;

3. The system limits the impact and scope of instability and cascading outages when they occur;

4. The system's facilities are protected from unacceptable damage by operating them within facility ratings;

5. The system's integrity can be restored promptly if it is lost; and

\footnotetext{
${ }^{7}$ Contributed by Art Diaz-Gonzalez, WAPA.

${ }^{8}$ Contributed by Yuri Makarov, PNNL.
} 
6. The system has the ability to supply the aggregate electric power and energy requirements of the electricity consumers at all times, taking into account scheduled and reasonably expected unscheduled outages of system components."

\subsubsection{Information needed for the analyses ${ }^{9}$}

Evaluation of transmission expansion project that is designed for variable generation is required to execute a system expansion project study. The primary objectives of this study should be to examine and establish acceptable reliability standards (risks) and profit margin. The study results will help develop calculated criteria to help determine whether to proceed with the transmission expansion. To provide a comprehensive analysis and assessment on local and regional benefits, various uncertainties, numerous anomalies and probabilities must be considered and weighed. Probabilistic assessments of multiple data input criteria provide an outcome representing both severity levels and occurrence-factors of multi-component failure events. An "appropriate combination of both severity and likelihood creates the indices that truly represent system risk" (Li 2005). The severity risk level of an outage may be altered based on the probabilistic simultaneous occurrence of additional or multiple outages. These, and a variety of other factors that must be considered and included in probabilistic models may include, but not be limited to, historical system power flow, load transfers, generation and network configurations, and state of system components. The key to developing an accurate composite model is highly dependent upon the historical data used, which should also include time period dependent factors (daily, weekly, monthly, and annually) incorporated into the calculations of the system being modeled.

There are numerous probabilities the process may calculate; some results may be more probable and others less so. Optimum results will only be limited by the data provided and modeled. On the contrary, too much data may prove detrimental with an apparent exponential burden of introducing too many variables. This can further add to the uncertainty of the model results, whereas full certainty (optimum risk factor) is never fully achieved. These dynamic stochastic factors represent the complexities of probabilistic transmission modeling, although the "Monte Carlo methodology has inherent limitations and full certainty is never quite achieved no matter how many samples are computed. The probability of serious algorithmic error is decreased with the number of samples, so that the calculation can be made more reliable than the data being used" (Skilling 1998).

In conclusion, probability theory applications are employed to achieve a final decision based on multiple variables of uncertainty. Probability calculus "is the only consistent calculus, and requires casting all uncertainty into the standard form of probability distribution. In data analysis, this uncertainty includes the original uncertainty about the unknown parameters we seek. The principle remains, that one should try to ask a question appropriate to one's data, but application of the principle requires care" (Skilling 1998).

\subsubsection{Probabilistic reliability analysis ${ }^{10}$}

Reliability assessment in power systems is an important problem to ensure safe and reliable operation of the systems. After several blackouts during the last decades, reliability assessment on large-scale power systems has attracted ever-increasing attention in the power system

\footnotetext{
${ }^{9}$ Contributed by Art Diaz-Gonzalez, WAPA.

${ }^{10}$ Contributed by Yuri Makarov and Jian Ma, PNNL.
} 
industry. Because of the probabilistic behavior associated with the system, the risk to largescale power systems cannot be totally avoided. To evaluate and manage the system reliability at an acceptable level is critically important for the planning, design, operation, and maintenance activities (Li 2005).

Deterministic methods usually are applied to reliability evaluation in most situations. The deterministic methods do not quantify uncertainties in the systems. Therefore, the information regarding how low or how high the system reliability can be is hard to obtain. It is required to develop a probabilistic method for evaluating system vulnerability.

Because of the probabilistic behavior of power systems, uncertainties associated with load demands result in the difficulty in achieving accurate load forecast. The consequences of random failures of power system components generally are outside the control of the operator. It is not easy to reflect all types of uncertainties associated with any possible consequences for probabilities. A reliability description based on probabilities alone does not necessarily provide a sufficiently informative picture of the reliability (Aven 2008). It is typically difficult to develop the severity function in most probabilistic reliability assessment problems (McCalley and Vittal 1998). Performance measures including load flow, steady-state voltage, transient voltage dip, and others are traditionally used approaches to quantifying impact. Therefore, the contingency probability is critical to probabilistic operations-based security assessment and related decisionmaking (Xiao et al. 2006).

To analyze the probabilistic reliability of a power system, two main categories of methods can be applied, i.e., the Monte Carlo simulation and the analytical approach. Some researchers combine the analytical method with contingency enumeration methods (Rei et al. 2006, Song et al. 2006). The Monte Carlo simulation method (Rei et al. 2006, Agreira et al. 2006, Hyungchul and Singh 2002) calculates the reliability indices by simulating the actual behavior of random failures in the power systems. Borges and Falcao (2000) described a sequential Monte Carlo method involving the application of multicomputer platforms. Other literature on simulation methodology can be found in Billinton and Li (1994) and many related papers (Billinton 1972, Allan et al. 1988, Allan et al. 1984, Allan et al. 1994, Allan et al. 1999).

The brute-force Monte Carlo simulation is not efficient and may lead to numerous runs without providing pre-knowledge information (Li 2005). Although variance reduction techniques can be applied to the simulations, Monte Carlo simulation is computationally very time-consuming, especially for large-scale power systems. Therefore, the technique that can effectively calculate probabilistic distributions with less computational expense is considered. High proficiency and accuracy may be achieved in analytical methodologies by programming with different algorithms, either complicated or simple (Propst 1995, Abdelaziz 1999). The analytical method calculates the reliability indices of power system composites based on mathematical models and solutions (Hailei et al. 2008, Ning et al. 2006, Chen et al. 1995, Billington and Allan 1996), and can significantly reduce the calculation time for large-scale power systems (Zhang and Lee 2004).

Many existing approaches assume that the random variables considered are independent. However, the dependence between the uncertainties of power injection should be considered for loads and for wind generation. The generalization of some of these methods for considering the dependence between random variables is very complex, or unfeasible. There are some proposals that consider this dependence, only between loads; such as in Leite da Silva et al. 
(1984), where it is modeled with a linear relation; and in Sanabria, and Dillon (1993), where the covariance has been taken into account in the equations.

\subsubsection{Frequently used probabilistic reliability indices ${ }^{11}$}

The most frequently used reliability indices are loss of load probability (LOLP) — which also is referred to as loss of load expectation (LOLE), expected unserved energy (EUE), system average interruption duration index (SAIDI), and system average interruption frequency index (SAIFI).

\section{Loss of load probability (LOLP)}

LOLP is the expected number of days in the year when the daily peak demand exceeds the available generating capacity. It is obtained by calculating the probability of daily peak demand exceeding the available capacity for each day, and adding these probabilities for all the days in the year. The index is referred to as hourly loss-of-load-expectation if hourly demands are used in the calculations, instead of daily peak demands.

\section{Expected unserved energy (EUE)}

EUE is the expected amount of energy curtailment per year. It is usually expressed in megawatt-hours.

\section{System average interruption duration index (SAIDI)}

The system average interruption duration index or SAIDI is the average number of minutes of interruption experienced by customers during the year.

\section{System average interruption frequency index (SAIFI)}

The system average interruption frequency index or SAIFI is the average number of interruptions experienced by customers during the year.

The additional useful indices are presented in Appendix 3.

High-impact low-frequency (HILF) events have recently become a renewed focus for risk managers. The information on HILF events and their impacts on electric power systems are discussed in Appendix 4.

\section{Some possible risk analysis methods (NERC 2010c)}

"As shown in Figure 2, risk curves can map to different risk measurement approaches:

1. The far right of the curve would employ a standard quantitative risk model

2. The middle (or early rise) would employ extreme value theory (EVT)

3. The HILF events (or late rise) can rely upon scenario analysis."

\footnotetext{
${ }^{11}$ Contributed by Yuri Makarov, PNNL.
} 


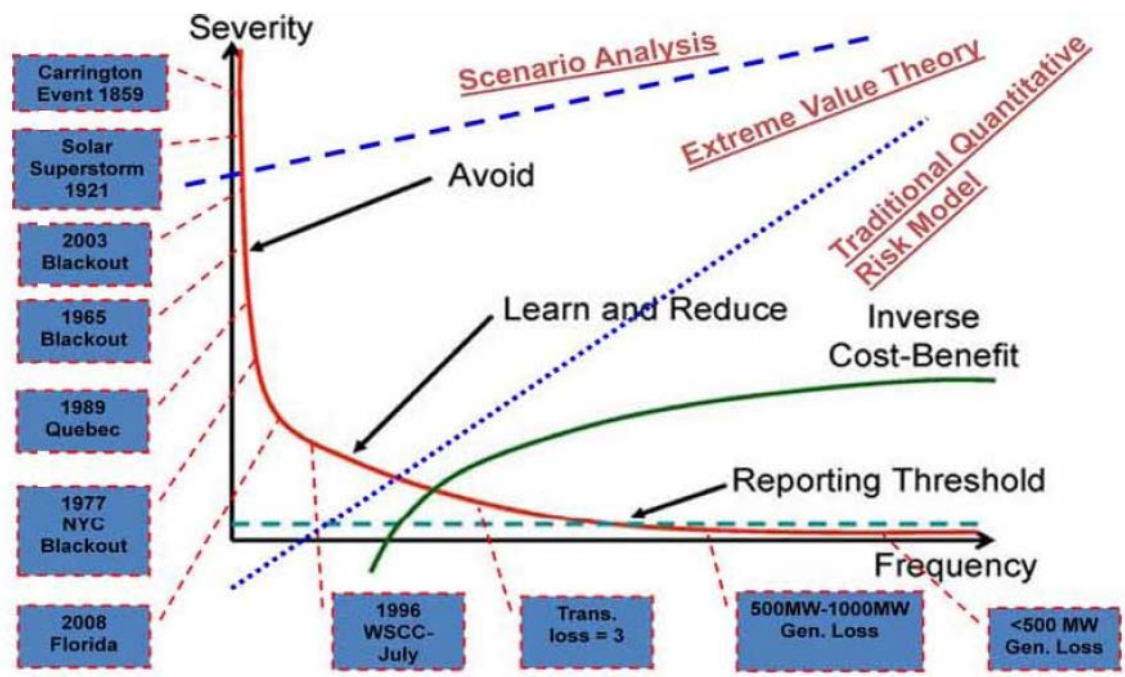

Figure 2. Some possible risk analysis methods depending on severity and frequency of events (NERC 2010c) ${ }^{12}{ }^{13}$.

"The red line depicts the events ranging from minor outages, to high-impact extreme events" (NERC 2010c).

"The line marked 'Reporting Threshold,' which is conceptually a level below where the severity does not warrant external reporting, because the impact is low (or outside the jurisdiction of the regulatory framework) or the system has operated as designed (also resulting in limited impact)" (NERC 2010c).

"There are events that occur with high frequency, but are quite small in terms of customer impact, as depicted in the green line. Many bulk power system events have generally no impact (and may be considered off-normal events ${ }^{14}$ or "operated as designed" type events) due, potentially in part, to the redundancy built in the bulk power system or other mitigating operations" (NERC 2010c).

More information on various extreme events analysis techniques can be found in Morgan et al. (2011).

"Scenario analysis ... is used to capture diverse opinions, concerns, and experience/expertise of key persons and represents them in a business model for measuring risk. Scenario analysis is a useful tool in capturing the qualitative and quantitative dimension of operational risk. Risk translates to where operational risk exposures exists, the severity of associated risks, identifies controls that are in place, and the type of control: damage, preventive, or detective. Cause and effect relationships can be captured with this method as well. The shortcoming of scenario analysis, however, is its subjectivity, which creates a potential for recording data inconsistently. Despite the shortcomings, the application of several divergent models can help develop a more confident convergent view of how much of an issue/response you need to prepare for in this event" (NERC 2010c).

\footnotetext{
12 NERC report.

13 Reused by permission of NERC

${ }^{14}$ More on off-normal events are available at the NERC RoP Section 808 and can be viewed at http://www.nerc.com/files/NERC_Rules_of_Procedure_EFFECTIVE_20100205.pdf.
} 


\subsection{Evaluation and provision of transmission capacity for VG integration}

This section discusses planning, cost allocation and nonincumbent developer reforms.

\subsubsection{Federal Energy Regulatory Commission (FERC) Order $1000^{15}$}

Order No. 1000 is a final rule that reforms the Commission's electric transmission planning and cost allocation requirements for public utility transmission providers. The rule builds on the reforms of Order No. 890 and corrects remaining deficiencies with respect to transmission planning processes and cost allocation methods.

Order No. 1000 establishes the following (FERC 2012a):

\subsubsection{Planning reforms}

"'The rule establishes three requirements for transmission planning:

- Each public utility transmission provider must participate in a regional transmission planning process that satisfies the transmission planning principles of Order No. 890 and produces a regional transmission plan. Local and regional transmission planning processes must consider transmission needs driven by public policy requirements established by state or federal laws or regulations. Each public utility transmission provider must establish procedures to identify transmission needs driven by public policy requirements and evaluate proposed solutions to those transmission needs. Public utility transmission providers in each pair of neighboring transmission planning regions must coordinate to determine if there are more efficient or cost-effective solutions to their mutual transmission needs" (FERC 2012a).

\subsubsection{Cost allocation reforms}

"The rule establishes three requirements for transmission cost allocation:

- Each public utility transmission provider must participate in a regional transmission planning process that has a regional cost allocation method for new transmission facilities selected in the regional transmission plan for purposes of cost allocation. The method must satisfy six regional cost allocation principles.

- Public utility transmission providers in neighboring transmission planning regions must have a common interregional cost allocation method for new interregional transmission facilities that the regions determine to be efficient or cost-effective. The method must satisfy six similar interregional cost allocation principles. Participant-funding of new transmission facilities is permitted, but is not allowed as the regional or interregional cost allocation method" (FERC 2012a).

\subsubsection{Nonincumbent developer reforms}

"Public utility transmission providers must remove from Commission-approved tariffs and agreements a federal right of first refusal for a transmission facility selected in a regional transmission plan for purposes of cost allocation, subject to the following limitations:

\footnotetext{
${ }^{15}$ Contributed by Pengwei Du, PNNL.
} 
- This does not apply to a transmission facility that is not selected in a regional transmission plan for purposes of cost allocation.

- This allows, but does not require, public utility transmission providers in a transmission planning region to use competitive bidding to solicit transmission projects or project developers.

- Nothing in this requirement affects state or local laws or regulations regarding the construction of transmission facilities, including but not limited to authority over siting or permitting of transmission facilities.

The rule recognizes that incumbent transmission providers may rely on regional transmission facilities to satisfy their reliability needs or service obligations. The rule requires each public utility transmission provider to amend its tariff to require reevaluation of the regional transmission plan to determine if delays in the development of a transmission facility require evaluation of alternative solutions, including those proposed by the incumbent, to ensure incumbent transmission providers can meet reliability needs or service obligations.

The Federal Energy Regulatory Commission issued Order No. 1000 to set a more comprehensive and coordinated approach to electric transmission planning in and among the various regions of the country, and to force the establishment of transparent and equitable methods of allocating the costs associated with improvements to the nation's transmission infrastructure. Order 1000 is completely consistent with previous commission policy and its efforts to promote integration of diverse resources into the energy market and foster competition among those resources. The new rule could make it easier for different types of generating sources to connect to the grid. In addition, natural gas-fired energy generators and storage projects that can provide alternatives to transmission or support integration of intermittent renewables may also benefit from the new regulations" (FERC 2012a).

\subsection{Better utilization of transmission assets}

Adequate transmission capacity is vital to integrating renewable energy resources into the grid. Many transmission networks are fully or almost fully utilized in some parts of the grid, while not in other parts. This is due to the fact that the transmission system has been developed for different flow patterns, based on the predictable generation and least fuel cost dispatch. The current dispatch patterns in the grid are increasingly driven by random impacts of the energy market and renewable generation. As a result, system stresses are becoming more variable, multidirectional, and dynamic. Some utilities have increased existing transmission capacity utilization by adjusting system security limits and by using new technology to quantify dynamic transmission limits. These goals can be achieved through advanced modeling of the system security region, and through new technical solutions, which are helping quantify transmission limits on specific grid elements.

\subsubsection{More accurate and adaptive characterization of the system security conditions}

Power transfers in contemporary power grids are increasingly limited by system security conditions, including thermal, voltage, and stability. They also are becoming more diverse and random with the increasing penetration of variable generation. As an alternative to various transmission system enhancements, transmission planners should consider a variety of approaches to help increase the available transfer capacity through a more accurate, adaptive, and flexible characterization of transmission limits. 


\subsubsection{Wide-area nomograms (aka, security region ${ }^{16}$ )}

Security region (SR) and security margin (SM) are important concepts that help ensure reliable power system operation (Makarov et al. 2009). A SR is defined as a $n$-dimensional set of nodal power injections (i.e., controllable generation and demand parameters) or voltage magnitudes and phase angles (i.e., state variables), for which the power flow equations and various security constraints are satisfied (Wu and Kumagai 1982, Kaye and Wu 1982). In general, the SR's boundary is a nonlinear hypersurface, composed of $n-1$ dimensional "manifolds," which depend on system configuration, operating conditions, enforced security constraints, and contingencies to be considered (Hiskens and Davy 2001, Makarov et al. 2000a, 2000b). The SR should reflect all constraints posed by all N-1 and all credible N-2 contingencies; that is, it is an inner intersection of individual SRs for those contingencies. A wide-area SR reflects system security conditions in a part of the interconnected system, including more than one Balancing Authority (BA).

\section{Security region vs. "typical" stress direction}

The SR concept contrasts the traditional security analysis approaches, where the system is gradually stressed from its base point along a "typical" stress direction, until it reaches the security region boundary (SRB). The stress is determined by a certain fixed pattern of load variation and generation redispatch. With the growing variability of dispatches, caused by the electricity markets and by increasing penetration of variable hard-to-predict energy resources and controllable demand, the idea of the single "typical" stress direction analysis becomes less and less relevant. The SR approach attracts more attention now because it considers multiple stress directions simultaneously, and it reflects mutual influence and possible uncorrelated variability of system parameters.

Nevertheless, there are several major difficulties, which are currently restricting practical applications of this important idea. They are multiple types of physically and mathematically complicated constraints reflected by a SR, the complex shape of the SRB, its high dimensionality, and computational efforts required to build a SR.

\section{Various types of security constraints}

The first difficulty is caused by various security constraints. The SRB can represent thermal, voltage, voltage stability (Su et al. 2002, Lee 2003, Dolloff et al. 2008), small signal (Li et al. 2002), or transient stability constraints (Zeng and Yu 2002, Zeng et al. 2005, Dong and Yu 2005, Yu et al. 2004). Existing tools address some of these aspects, but not all. It is impractical to incorporate all these constraints within a single software product. At the present time, such a software product does not exist. Furthermore, practicing engineers trust certain existing software products, gain experience with them, use multiple procedures based on these tools, and they do not want to replace their existing, now-familiar technology just because of a single, attractive idea of the SR analysis.

\section{Analytical description of security region}

The second difficulty lies in an analytical description of the multidimensional and nonlinear SRB. The analytical description involves certain mathematical expressions, which can be used by a computer program to automate answers to questions such as:

- Is the system state secure?

\footnotetext{
${ }^{16}$ Contributed by Yuri Makarov, PNNL.
} 
- How far is the system state from insecurity?

- What type of problems could be caused?

- Which elements of the system cause insecurity?

- What needs to be done to improve the security margin?

A simple visualization of the SRB is not sufficient for these purposes. For instance, the physical and operational margin (POM) software (Dolloff et al. 2008) incorporates thermal, voltage, steady state, flow gate, and transient stability constraints; and it provides two- and threedimensional plots of the SRB in coordinates of selected system parameters. At the same time, the tool has not been expanded yet to address a general case of multiple critical parameters, because it is limited to visualization purposes and because it does not contain the analytical engines needed to describe the SRB. A widely used approximation is the one that used to produce linearized operating nomograms (McCalley et al. 1997), which consists of piecewise linear contours on a plane of two critical parameters. If three parameters are involved, the nomogram becomes a family of contours; each of them corresponds to a certain value of the third parameter. A natural extension of the linearized nomograms for four or more dimensions can be made using hyperplanes $-n$-dimensional planes that are defined in the parameter space by approximating the SRB. Some of these efforts are described in Parashar et al. (2008) and Parashar et al. (2007), for voltage stability SRB approximations, and in Zeng and Yu (2002), Yu et al. (2004), and Djukanovic et al. (1993) for transient stability SRB approximation. Nonlinear approximations were successfully used to represent SRB in Russia, where quadratic inequalities were employed to describe SRB (Sovalov and Semenov 1988).

\section{Large dimensionality of security region}

Large dimensionality is the third difficulty for the wide-area SR analysis. With multiple dimensions, the number of system stresses needed to build an SRB approximation with reasonable accuracy increases dramatically. Because of this problem, the number of simultaneously considered parameters is usually significantly reduced in practical applications. For instance, the real-time voltage stability analysis tool, which has been used by BC Hydro for several years, combines voltage stability and thermal constraints, and uses a linearized formulation of the SRB (BC Hydro 2006). At the same time, only three critical parameters are considered. In China, Prof. Y. Yu et al. proposed to use the "critical cut-set space" to solve the dimensionality issue (Su et al. 2002). Similar ideas can be used by limiting the number of analyzed dimensions to several descriptor parameters, such as power flows in certain system paths, zonal generation and load.

\section{Computational effort}

The fourth difficulty of computation intensity comes from the variety of system stresses needed to explore the whole SRB in a multidimensional space. To minimize computations in real time, a pre-calculated SR can be used. Nonetheless, for a multidimensional SR, even the offline SR building procedures require significant time. For instance, these calculations by BC Hydro take several months of highly qualified engineering work (BC Hydro 2006).

\section{Situation awareness and actionable information}

A security assessment tool should be able to provide the current security margin, that is, to answer the questions: Is the system state secure? How far is the system state from insecurity? Various approaches were proposed for this purpose. For instance, many voltage stability indices and methods have been introduced for example, in Hiskens and Davy (2001), Löf et al.(1992), 
Tamura et al. (1983), Rosehart et al. (2005), Karystianos et al. (2007), and Kataoka and Shinoda (2005). For an example of transient stability margin evaluation methods, see Fouad and Vittal (1992). The SR approach helps to evaluate the security margin without performing time-consuming calculations.

Power system grid planning engineers also need answers to the questions: Which elements of the system cause insecurity? What needs to be done to improve the security margin? Several approaches to calculate the sensitivity of the stability margin with respect to system parameters have been proposed (Greene et al. 1997, Ruiz-Vega et al. 2004, Begovic and Phadke 1992, Greene et al. 2002, Li and Venkatasubramania 2004, Castellanos and Messina 2008).

Nevertheless, it remains challenging to quickly estimate the effect of the change in system conditions on the system security margin - while considering various types of security constraints and simultaneous interactions between multiple critical parameters, such as path flows distributed in a wide area.

Examples of using wide-area nomograms in practical applications are presented in Appendix 5.

\subsubsection{Dynamic rating ${ }^{17}$}

To avoid exceeding the thermal constraints of transmission lines, utilities have set maximum power flow limits for most transmission lines. These static thermal limits are based on the worstcase scenario taking into account environmental conditions. If the maximum power flow on a transmission line exceeds its limits under these conditions, the line could sag to the point where it would come into contact with trees, trucks, boats, or other nearby hazards. Because the environmental conditions are almost never as unfavorable as those used to calculate the static thermal limits of a transmission line, the true maximum capacity of a transmission line is underestimated most of time (Holman 2011).

Elements of the theory behind the transmission line ratings, types of dynamic rating methods, and potential implications of power and renewable industry are discussed in Appendix 6.

\section{Static Ratings}

Overhead transmission line conductors can be rated in accordance with IEEE (2006).

The steady-state thermal rating is the loading that corresponds to maximum allowable conductor temperature assuming thermal equilibrium. Many weather variables affect the conductor temperature: 1) wind speed and direction, 2) ambient air temperature, 3) solar radiation, and other variables. A thermal line rating is a function of these weather conditions (Giberson 2009).

The static thermal rating can be taken as the current carried by a given transmission line conductor, resulting in the maximum allowable conductor temperature for a fixed and constant set of conservative (worst-case) weather conditions over an extended period of time, days, months or years, neglecting weather variability (Giberson 2009).

The thermal limit/current capacity/ampacity of overhead transmission lines is limited by system stability concerns, temperature limit of the conductor material, and thermal sag of the conductor. Therefore, the transmission line thermal rating is the highest current at which a line can be

\footnotetext{
${ }^{17}$ Contributed by David Tovar, (El Paso Electric) EPE, and Jian Ma, PNNL.
} 
operated without violating safety codes (for sag/clearance limits), maintaining the integrity of the line materials, or operating reliably (usually determined by study results) (Seppa 2007). The thermal rating of an overhead transmission line is usually the lower of the maximum current that a circuit can carry: 1) without exceeding its sag temperature (temperature, at which the legislated height of the phase conductor above ground, clearance, is met) or 2) the annealing onset temperature of the conductor (Yip et al. 2009). Current clearance requirements, calculated at the maximum conductor operating temperatures, are listed in the latest National Electrical Safety Code (NESC) ${ }^{18}$ and the latest, applicable state codes.

In the electric utility industry, various ampacity levels are chosen to ensure acceptable conductor loss of life and code mandated clearance limits; these levels are called normal and emergency ratings. The normal static thermal rating can be chosen as the rating, adjusted for a fixed set of conservative weather conditions that will allow maximum equipment loading without incurring loss of life above design criteria. For this calculation, maximum allowable conductor temperature for this condition must be provided. The emergency static rating (or ratings), can be chosen as the rating(s), adjusted for a fixed set of conservative weather conditions, that will allow loss of life, or loss of tensile strength in excess of design criteria, and to some percentage of loss of life. For this calculation, maximum allowable conductor temperature for this condition must be provided.

Note that the ampacity rating on some transmission lines possibly can be based on a bottleneck element (element with lower current ratings than the rating of the line) that is a part of the line circuit. For example, series compensation elements, wave traps, connectors, splices, and/or multiconductor lines can lower the thermal limit of overhead transmission lines.

\section{Dynamic rating}

The increased demand on transmission system utilization can be partially met by using dynamic ratings of overhead transmission conductors. Dynamic rating (also called real-time rating) is a rating developed in real time (or near real time), and is based on actual weather and loading conditions to develop accurate ratings of components - instead of using the fixed conditions used in static rating calculations. In the case of overhead transmission lines, the key elements in the development of a dynamic thermal rating are the conductor composition and construction, its temperature, the ambient temperature, the wind speed, and its direction. The dynamic thermal rating of an overhead conductor may be defined as the steady load that produces the maximum conductor operating temperature, computed on an instantaneous basis for actual weather conditions (Holbert and Heydt 2001, Mayadas-Dering 2009).

Dynamic ratings provide higher line capabilities most of the time. Usually, real-time ratings exceed static ratings by at least 10-15 percent for 95 percent of time, and by 20-25 percent for 85 percent of operational time (Seppa 2007). Dynamic line ratings may be able to provide a system operator with an overhead transmission line's real-time, power-carrying ability, while not exceeding design limits (e.g., conductor temperature, line sag/clearance limitations, and the "next limiting element" (Shirmohamadi 2002). These dynamic ratings are well suited for operational line ratings, where conditions are constantly changing.

\footnotetext{
${ }^{18}$ IEEE Standards Association, National Electrical Safety Code, Website: http://standards.ieee.org/about/nesc/index.html
} 
When transmission line ampacity is increased because of an increased real-time rating, it is important to evaluate the thermal effects of the conductor (loss of tensile strength and permanent elongation) and conductor sag (Brennan et al. 2008). Failure to monitor thermal effects can lead to severe loss of the conductor's tensile strength; creep, which is a nonrecoverable deformation; and a safety breach (Brennan et al. 2008). A negative consequence of transmission line overheating is conductor sag, which can violate National Electric Safety Code (NESC) clearance regulations (Brennan et al. 2008). Often, a line's rating is based on the ability of the utility to maintain a safe clearance between energized conductors and the ground, trees, vehicles, and other objects directly below the line, as set forth in regulations such as the NESC (NESC) (Forbes et al. 2002).

\section{Dynamic vs. static rating}

Unlike a static rating, a dynamic rating is valid for a limited time period. When static overhead transmission line ratings are calculated, conservative assumptions of worst-case expected fixed ambient conditions (low-wind value and high-summer period temperature assumptions) are made. There also is usually a buffer in design clearance calculations using these fixed conditions (Forbes et al. 2002). With static ratings, if any one factor in the assumptions of worstcase expected fixed ambient conditions used to calculate a static overhead transmission line rating is too conservative (such as the wind speed value is too low), the static rating for this line will be conservative. For example, it is estimated that for a given conductor, under a set of initially fixed conditions, a drop of $10^{\circ} \mathrm{C}$ can result in about an 11 percent increase in conductor current rating; the absence of solar radiation in the middle of the night can result in about an 18 percent increase in conductor current rating; and a wind speed increase of $1 \mathrm{~m} / \mathrm{s}$ can result in at least a 35 percent increase in conductor current rating (Stelmak 2009). Also, when applying static ratings, no attempt is made to determine the equipment temperatures. Therefore, it is not known what the conductor current rating actually is at any one point in time; other than at the fixed conditions being assumed for the static rating calculations. One of the reasons that these conservative assumptions are made is that the overhead transmission line ratings are often used in planning studies modeling peak load conditions, where the assumptions may be valid (worst-case, peak load planning).

With equipment infrastructure aging, permitting difficulties, and difficulties constructing new facilities, the maximum use of existing facilities is a priority. This is encouraging the electrical power industry to reevaluate existing equipment ratings. The use of dynamic ratings and realtime monitoring of transmission lines can be key to real-time evaluation of overhead transmission line ratings and capacity.

\section{Dynamic rating implementation}

Dynamic line rating implementation is calculating the line's ampacity in real time from actual weather conditions and physical parameters of the conductor. In dynamic line rating, it is essential to gather data about the line's present condition. The data is then used to calculate the new and updated ampacity (Brennan et al. 2008).

The updated ampacity data can then be transmitted to a control center. Calculation of normal, emergency, and transient line ratings (given specific conductor type/bundling) can be made, and this information can be posted at system operator monitors. Real-time rating systems can operate in a control center and can provide system operators with alarms only when specific predetermined conditions occur, or with a queue from the operator of present conditions (on a specific line being monitored on a real-time basis) (Seppa 2007). System operators simultaneously can monitor static ratings, dynamic ratings, and actual line loading in real-time 
simultaneously, on the same screen. Historical trending of dynamic ratings or recent line loading, if desired, can also be displayed for guidance.

Dynamic line rating requires additional equipment to be added to the transmission line and an information link to be made to the transmission control system, but it uses the existing line in the existing right of way. Some technologies require the transmission line to be taken out of service as they are installed or calibrated, which can cause disruption, particularly because the technology is often installed on lines that are very heavily loaded. One tradeoff that must be considered when evaluating dynamic rating and other technologies is that the losses in transmission lines increase as the square of the current through them. Therefore, there is a penalty for running high currents through transmission lines, which makes "high current" technologies more attractive when those higher currents will only be required on a temporary basis, such as during periods of peak demand or when other transmission lines fail (Holman 2011).

\section{Operating with dynamic ratings}

When transmission line flows are limited by their thermal capacity, rather than voltage or stability limits, dynamic thermal ratings offer many advantages and challenges compared with the traditional static thermal ratings.

Because dynamic thermal ratings normally are higher than static ratings, some advantages of their use are:

- Increased reliability and safety by monitoring real time rating under pre-contingency and post-contingency conditions.

- Evaluate line sag and prevent exceeding clearance/sag limits.

- Potential increase in real-time transfer capacity.

- Potential savings through more efficient use of equipment, fewer new lines, less land use, and decreased environmental impacts.

- Better evaluation of static line rating assumptions.

Some potential disadvantages of using dynamic thermal ratings are:

- All potential disadvantages associated with operating with more loaded lines: higher current, higher conductor temperature (sag, annealing, loss of life), etc.

- All potential disadvantages associated with setting up, costs of equipment, and maintenance for operating at higher ampacity: monitoring equipment, setting up costs, personnel time and costs, and evaluation of all lines.

- Need close monitoring for regulatory and technical criteria limits.

\subsection{Transmission technologies}

The relative costs per MW-km of different electric power transmission technologies are presented in Appendix 7. 


\subsubsection{Ultra-high voltage $\mathrm{AC}^{19}$}

"From the advent of electrical transmission, alternating current (AC) has established itself as leading technology in electrical networks. Its advantage lay in the possibility of using transformers to raise it to higher voltage levels, facilitating economical transmission. Both AC and direct current (DC) generators produce electricity at a relatively low-voltage level. If this voltage were used for transmission over long distances, high and prohibitively expensive losses would ensue. AC technology is also flexible when connecting different locations to form an electric grid, permitting a robust and reliable electric supply to consumers.

The development of AC systems has paralleled continuing increases in transmission voltage. When power consumption is low, voltage also can be low. Typically, doubling the voltage quadruples the power transfer capability. Consequently, the evolution of grids in most countries is characterized by the addition of network layers of increasingly higher voltages.

Short AC lines have high-power handling capability. The capability is dependent on the voltage and the thermal rating of the conductors. Longer lines have a higher impedance, and this reduces the power transfer capability. The simplified equation for transfer of active power is:

$$
P=\frac{V_{1} \cdot V_{2}}{X} \sin \delta
$$

Where $P$ is the active power, $V_{1}$ and $V_{2}$ the voltage at each end of the line, $\delta$ the phase angle between the two ends and $X$ the line impedance. As the length of the line increases, the impedance of the line increases with it. For the transfer power to be maintained, the angle $\delta$

problems with dynamic stability can be encountered. The best way to overcome this problem is to reduce the impedance by series compensation. This can be done without significant problems up to a compensation of around 70 percent. At higher levels of compensation, the system will be less robust" (Asplund 2007). Figure 3 shows transmission capability in megawatts of $A C$ lines with 50 percent compensation.

\footnotetext{
${ }^{19}$ Contributed by Pavel Etingov and Yuri Makarov, PNNL.
} 


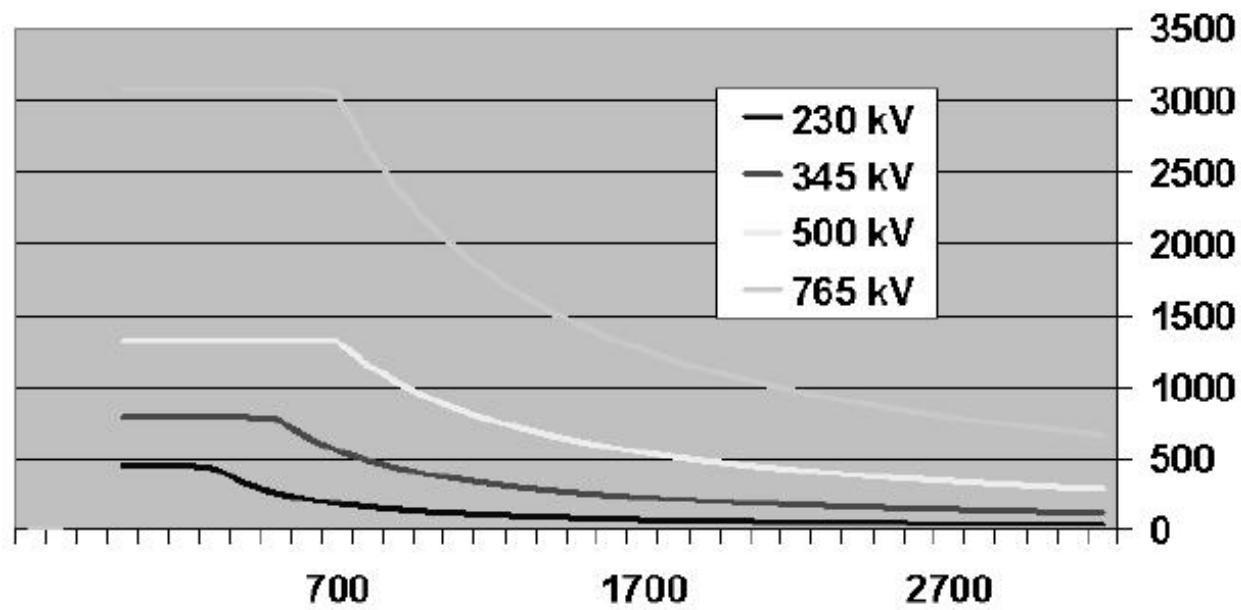

Line length in $\mathrm{km}$

Figure 3. Transmission capability in megawatts of AC lines with 50 percent compensation (Asplund and Williamson 2007). ${ }^{20}$

The electric power transmission at $1,000 \mathrm{kV}$ and above $\mathrm{AC}$ voltages is known as ultra-high voltage (UHV) AC transmission. In order to meet the growing of power load, to carry out long distance and bulk capacity power transmission, Russia (the former USSR), Japan, United States of America (USA), Italy, Canada, and Brazil began study on the UHV transmission relevant technologies in 1960s and 1970s (Huang et al 2009).

\subsubsection{Advantages of UHV AC:}

- Improved characteristics of UHV transmission: "The UHV transmission has obvious advantages of improving transmission capacity, increasing power transmission distance, reducing line losses, lowering project investment, saving line corridors" (Huang et al 2009).

- Increased transmission capacity: "The UHV transmission can increase the transmission capacity. The natural transmission capacity of a 1,000-kV AC circuit is about $5 \mathrm{GW}$, and that is approximately 4-5 times that of a 500-kV AC transmission line" (Huang et al. 2009).

- Increased transmission distance: "The UHV transmission could increase the economic power transmission distance. A 1,000-kV AC line can economically transmit power distances of $1,000 \mathrm{~km}$ to $2,000 \mathrm{~km}$ " (Huang et al 2009).

- Reduced transmission losses: "If the conductor cross-section and transmission power are regarded as constant, the resistance loss of a 1000-kV AC power line is 25 percent that of the 500-kV AC power line" (Huang et al. 2009).

- Reduced cost: "At the same conditions, the resistance loss of the 1,000-kV AC line is only 25 percent of the 500-kV AC line, and the project investment, etc., can be saved.

\footnotetext{
${ }^{20}$ Reused by permission of IEEE.
} 
The cost per unit of transmission capacity of $1,000-\mathrm{kV}$ AC transmission scheme is 73 percent of 500-kV AC scheme" (Huang et al. 2009).

- Reduced land requirements: "UHV AC transmission has obvious advantages in reducing the land occupation of the line. A 1,000-kV AC power line saves 50 percent to 66 percent of the corridor area required by 500-kV AC lines in transmitting the same capacity" (Huang et al. 2009).

- Isolation requirements: "Insulation for UHV AC is dominated mainly by switching overvoltages and the required insulator lengths phase-to-ground are from 7 to 9 meters for a 1000-kV system. These fall within the limits of present feasibility" (Huang et al. 2009).

- "The situation, however, is different in the case of UHV DC, with insulator design dominated mostly by pollution requirements, which for 800-kV DC could lead to insulator dimensions of 10 to 12 meters or higher. These units would be far more difficult to manufacture, handle, and transport" (Huang et al. 2009).

\subsubsection{Challenges of UHV AC}

"There are several challenges involved in building UHV lines, and new equipment needing to be developed includes transformers, breakers, arresters, shunt reactors, series capacitors, current and voltage transformers, and connecting and ground switches. There are also special requirements in the domain of control and protection. At single-phase Earth faults, the challenge is to clear the fault without opening the breakers of all three phases. The problem lies with the high capacitive current generated by the operating phases that flows into the fault. This can be achieved with the help of tuned reactors that minimize the induced current" (Asplund 2007).

\subsubsection{UHV AC and renewable generation (Whitney 2011)}

"The United States is incredibly rich in potential renewable power resources. Just cherry-picking the low-hanging fruit of high-value renewable resources with the lowest electricity generation costs finds nearly the entire country within reach of multiple renewable power sources, if we can establish a UHV transmission grid. As an additional benefit, power from the overlapping renewable resources would even out generation fluctuations, addressing once and for all renewable energy intermittency issues.

- Solar generation. We have high solar resources in the Southwest states of New Mexico, Colorado, Utah, Nevada, California, and Arizona. UHV-AC brings 75 percent of the country within range of these solar resources, from Seattle in the north to Chicago and Nashville in the east. UHV-DC puts the entire country within range with the exception of New England.

- Wind generation. High-value wind resources extend from North Dakota to Texas including South Dakota, Nebraska, portions of lowa and Montana, Kansas, and Oklahoma. UHV-AC transmission from Great Plains wind farms would reach all of the U.S., with the exception of southern Florida and New England. UHV-DC lines would bring low-cost wind power to the entire U.S.

- Hydropower generation. Hydropower resources are located throughout the entire nation, but are especially rich everywhere except for the Great Plains and Florida. UHV-AC transmission would bring the hydropower to the entire country. 
- Enhanced geothermal generation. High-value, favorable, deep, enhanced geothermal systems (EGS) sites are located throughout the western states, with particularly favorable resources in Colorado, Utah, Nevada, Idaho, Oregon, and Northern California. Much like high-insolation Southwestern solar resources, UHV-AC transmission would bring 75 percent of the country within reach of low cost geothermal power. UHV-DC lines could reach every region except for New England" (Whitney 2011).

National and international experience in UHV transmission is presented in Appendix 8.

\subsubsection{Compact AC lines}

"Overhead line transfer distance and/or capacity also can be increased by observing some new rules for the line design, as follows:

1. Employ more subconductors ${ }^{21}$ in each line phase: The greater the transmitted power or line length, the greater the number of subconductors required.

2. Place all subconductors in split phases in order to provide the maximum permissible field strength on each subconductor (subconductors of a phase can be placed in a circle with unequal subspace distances).

3. Phase dimensions increase when the number of subconductors increases. For optimum subconductor placement, phase dimensions can be decreased by:

- modifying the tower structure to exclude the tower's grounded elements from the interphase space;

- finding the optimum phase dispositions within the space;

- allowing larger interphase overvoltage limitations to decrease interphase air gaps; and

- using interphase insulation spacers to decrease wind-induced interphase distance changes.

Implementing the aforementioned rules results in increased surge impedance loading (ISIL)" (Evdokunin 2004). "For example, for a 220-kV line with three subconductors per phase, it is possible to obtain inductive impedance of 0.15 to $0.17 \mathrm{Ohm} / \mathrm{km}$, as compared to $0.4 \mathrm{Ohm} / \mathrm{km}$ for a standard $50-\mathrm{Hz}$ line. For a $500-\mathrm{kV}$ line with six or seven subconductors, inductive impedance can be lowered by half, in comparison with ordinary lines" (Evodokunin 2004).

"Lowering the inductive impedance of long-distance transmission lines in this manner facilitates a decrease in the power angle between the line terminals and ensures steady-state stability requirements without series capacitive compensation" (Evdokunin 2004).

In addition, this new type of line provides significant economic advantages in comparison with conventional lines, because of reduced power losses, inter-phase dimensions, line right-of-way width, and saving on construction and real estate costs (Evdokunin 2004).

\footnotetext{
${ }^{21}$ Bundle conductors are meant in the reference.
} 
"For example, in a 330-kV line (Russia) with four subconductors per phase - placing more subconductors in the ISIL line phases translated into a 5 percent increase in line construction costs. However, ISIL lines do not require series capacitive compensation, so there was a 35 percent decrease in line construction costs. But because the ISIL structure generally decreases linear inductive impedance while increasing linear capacitive impedance, it was desirable to install shunt reactors for compensation of the latter. This added 5 percent to the construction cost. But the net result of these several modifications was a savings of 15 percent over ordinary transmission line construction" (Evdokunin 2004).

\subsubsection{HVDC line design considerations ${ }^{22}$}

The content of this section is mainly based on references Huang et al. (2002), Lescale et al. (2008), Bahrman et al. (2003) and Rudervall et al (2002).

The fundamental process that occurs in an HVDC system is the conversion of electrical current from AC to DC (rectifier) at the transmitting end, and from DC to AC (inverter) at the receiving end (Rudervall et al. 2002).

\section{Converter design}

"HVDC transmission systems are based on either Voltage sourced converter (VSC) or conventional line commutated converter (LCC) an alternative to HVAC connections" (Alstom Grid 2010).

"Conventional HVDC transmission employs line-commutated, current-source converters (LCC) and requires a synchronous voltage source in order to operate. The conversion process demands reactive power from filters, shunt banks, or series capacitors, which are part of the converter station. Any surplus or deficit in reactive power must be accommodated by the AC system. This difference in reactive power needs to be kept within a given band to keep the AC voltage within the desired tolerance" (Bahrman et al. 2003). "Natural commutated converters are most used in the HVDC systems as of today. The component that enables this conversion process is the thyristor, which is a controllable semiconductor that can carry very high currents $(4,000 \mathrm{~A})$ and is able to block very high voltages (up to $10 \mathrm{kV})$. By means of connecting the thyristors in series, it is possible to build up a thyristor valve, which is able to operate at very high voltages (several hundred $\mathrm{kV}$ ). The thyristor valve is operated at net frequency $(50 \mathrm{~Hz}$ or 60 $\mathrm{Hz}$ ) and by means of a control angle it is possible to change the DC voltage level of the bridge. This ability is the way by which the transmitted power is controlled rapidly and efficiently" (Rudervall et al. 2002).

Capacitor commutated converters (CCC). "An improvement in the thyristor-based commutation, the CCC concept is characterized by the use of commutation capacitors inserted in series between the converter transformers and the thyristor valves. The commutation capacitors improve the commutation failure performance of the converters when connected to weak networks" (Rudervall et al. 2002).

Forced commutated converter or voltage source converters. "This type of converter introduces a spectrum of advantages, e.g., the feed of passive networks (without generation), independent control of active and reactive power, and power quality. The valves of these converters are built up with semiconductors with the ability not only to turn-on but also to turn-off. They are known

\footnotetext{
${ }^{22}$ Contributed by Yuri Makarov, PNNL.
} 
as voltage source converters. Two types of semiconductors normally are used in the voltage source converters: the GTO (gate turn-off thyristor) or the IGBT (insulated gate bipolar transistor). Both have been in frequent use in industrial applications since early 1980s. The VSC commutates with high frequency (not with the net frequency). The operation of the converter is achieved by pulse width modulation (PWM). With PWM, it is possible to create any phase angle and/or amplitude (up to a certain limit) by changing the PWM pattern, which can be done almost instantaneously. Thus, PWM offers the possibility to control both active and reactive power independently. This makes the PWM voltage source converter a close-to-ideal component in the transmission network. From a transmission network viewpoint, it acts as a motor or generator without mass that can control active and reactive power almost instantaneously" (Rudervall et al. 2002).

New "VSC-based HVDC transmission utilizes several important technological developments:

- High voltage valves with series-connected IGBTs;

- Compact, dry, high-voltage DC capacitors;

- High capacity control system; and

- Solid dielectric DC cable.

A special gate unit and voltage divider across each insulated-gate bipolar transistor ${ }^{23}$ maintain an even voltage distribution across the series-connected IGBTs. The gate unit not only maintains proper voltage sharing within the valve during normal switching conditions, but also during system disturbances and fault conditions. A reliable short circuit failure mode exists for individual IGBTs within each valve position."

"The following factors make VSC-based transmission attractive:

- Independent control of reactive and active power;

- Reactive control independent of other terminal(s);

- Simpler interface with AC system;

- Compact filters;

- Provides continuous AC voltage regulation;

- No minimum power restriction;

- Operation in extremely weak systems;

- No commutation failures;

- No restriction on multiple in-feeds

- No polarity reversal needed to reverse power;

\footnotetext{
23 "The insulated gate bipolar transistor or IGBT is a three-terminal power semiconductor device, noted for high efficiency and fast switching. Because it is designed to turn on and off rapidly, amplifiers that use it often synthesize complex waveforms with pulse width modulation and low-pass filters. The IGBT is used in medium- to high-power applications, such as switched-mode power supply, traction motor control, and induction heating. Large IGBT modules typically consist of many devices in parallel and can have very high current handling capabilities in the order of hundreds of amperes with blocking voltages of 6,000 V, equating to hundreds of kilowatts."[Wikipedia]
} 
- Black-start capability;

- Variable frequency; and

- HVDC light cable - economic extruded polymer" (Bahrman et al. 2003).

The characteristics of VSC-HVDC are discussed in Appendix 9.1.

\section{Selection of rated voltage, current and project configuration.}

"The motivation to increase the voltage level for transmission of power over long distances is there as it would reduce the overall cost of transmission of large quantities of power. Up to a power of about 3,000-3,500 MW, a single bipole operating at $\pm 500 \mathrm{kV}$ might be sufficient. But for higher power levels. there are likely to be sufficient reasons to either increase the operating voltage or number of bipoles or both. The reasons may be due to the cost of transmission line, cost of losses, transportation limitations of converter transformers, approaching current limits of thyristor valves, etc." (Huang et al. 2002). Additional information on the selection of voltage, current and project configuration is provided in Appendix 9.2.

\section{Selection of rated current.}

"For UHVDC projects, depending upon the rating of the project, the continuous current rating may approach 3,500-4,000 amps. Over and above the rated current, the project would also require overload ratings. Important considerations with regard to the current rating of the project would be with respect to:

- Losses on DC line;

- Overload capability of thyristors and the project;

- Cooling equipment;

- Tap changer range due to higher voltage drop on the line; and

- Ground currents during pole outages" (Huang et al. 2002).

Additional information on the selection of rated current is provided in Appendix 9 (Section 3).

\section{Line length}

"Primarily, the length of the line affects the exposure for various weather conditions, such as lightning storms and fog. It also impacts its exposure for other external conditions such as brush fires. However, super-low frequency induced voltage due to geomagnetic storms do not impact DC lines as the induced voltage is negligible compared with the converter voltages.

The large length for UHVDC also has an effect on the travel time between the terminals both for main circuit transients and telecommunication signals. The electric wave traveling speed in an overhead line is close to the speed of light. The speed of light in an optic fiber is not faster than the speed of light divided by 1.5. This means that it takes $3.3 \mathrm{~ms}$ before the rectifier sees the effect of a fault in the inverter if the line has a length of $1,000 \mathrm{~km}$, and it takes $6.6 \mathrm{~ms}$ until the inverter sees any response from the rectifier, in addition to the rectifier response time. During these $6.6 \mathrm{~ms}$, the inverter only sees the surge impedance of the line of around $300 \Omega$. The corresponding surge current is $2.7 \mathrm{kA}$ at a DC line voltage of $800 \mathrm{kV}$. The additional response time due to the wave traveling time in the optic fibers is $5 \mathrm{~ms}$. Thus, the additional time delay of the response from the other station is $10 \mathrm{~ms}$. This time delay may have minor impact on the 
restoration of the system after a disturbance, but it has to be considered during the design for not having a number of series-connected questions/answers between the terminals. Furthermore, this time delay is very important at design of the regulators for any type of damping regulators" (Lescale et al. 2008).

\section{Telecommunication}

"With a number of terminals and/or converters operating in parallel, the importance of the telecommunication for coordination of the multi-terminal UHVDC system increases. The reason is the number of possible configurations in operation after a disturbance or an outage. Even if the equipment is protected against damages, lack of coordination and inter-terminal actions may lead to prolonged disturbances or loss of all converters connected to the same pole line. This is especially critical at loss of one parallel inverter during high load, as the pre-fault DC current from the rectifiers will be too high for the remaining inverter(s). In practice it is not possible to change the load without telecommunication. Even manual change of the load flow requires communication between the operators" (Lescale et al. 2008).

\section{Complexity}

"In any HVDC project a major emphasis is on a design to achieve:

- Long, continuous operations without fault;

- Ability to recover from faults quickly;

- Only partial and acceptable loss on major faults; and

- Well integration in the AC system.

The long, continuous operation of the project is basically achieved by proper design, strict quality control, adequate redundancy, efficient operator interface and proper installation, to mention a few things. One additional thing that has the potential to affect long, continuous operation is the inherent complexity of a project. In spite of paying full attention to all the above aspects, it is still desirable to keep the number of equipment installed as low as possible. For instance, having two 12-pulse groups in series adds more equipment in the ac switchyard, valve halls and DC switchyard and is somewhat less reliable, as compared to a much more common and utilized one 12-pulse configuration per pole" (Huang et al. 2002).

\section{Partial outages}

"When there are two series 12-pulse groups per pole, there shall be certain faults within the converter station that may require shut down of only one of the two 12-pulse groups. Under these circumstances, the loss of power in case of two series groups per pole shall be lower compared to the case when there is only one 12-pulse group per pole. However, it is likely that while designing the project, the contingency of faults on DC line that require shutting down one entire pole of the station has to be considered, and therefore, even when there are two twelve pulse series groups per pole, the outage of a pole has to be considered for the design of the project" (Huang et al. 2002).

\section{Reduced voltage operation}

"The reduced voltage operation is mostly anticipated to ride-through flashover problems on the DC line or DC switchyard. These flashovers may be caused by reduced insulation strength or environmental conditions like fog, pollution, etc. The reduced voltage operation is achieved through a combination of tap changer operation and firing angle control. In case of two 12-pulse 
groups per pole, there is an additional flexibility to reduce the operating voltage by shutting down one 12-pulse group, which shall bring down the voltage to half. However, this shall also reduce down the power by half. This flexibility of shutting down one of the two 12-pulse groups, however, may be of limited use since the normal requirements of improving flashover problems by operating in a reduced voltage are met by bringing down the voltage to about 80 percent of the nominal voltage or, at most, to 70 percent of nominal voltage" (Huang et al. 2002).

\section{Reliability}

"Reliability involves equipment with a proper and good strategy for redundancy and supervision of control and measurement systems. Furthermore, for large and complex systems, a throughout failure mode effect analysis is needed to identify and redeem common mode failures, which may affect more than one converter unit. In this aspect, it must be remembered that the AC system is common for all converters in at least one terminal. Thus, an appropriate coordination between the AC side and DC side protections is very essential. The auxiliary power supply may also be common for more than one converter" (Lescale et al. 2008).

\section{Transient stability}

"Regarding transient reliability, fast detection and isolation of faulty equipment are very essential. It is also important that the fault clearance actions do not trip more equipment than absolutely necessary. This is also valid for isolation of the faulty DC line section in case of a persistent DC line fault. Utilization of overload capability of the remaining equipment reduces the consequences at an outage. However, it must be assured that there is no increased risk for outage of additional equipment" (Lescale et al. 2008).

\section{Availability}

"Regarding availability, it is important that all maintenance can be performed with absolutely minimum of outage of converters and/or lines" (Lescale et al. 2008).

\section{Electric and magnetic fields}

"Considering the environmental impact, neither the electrical field nor the magnetic fields from the UHVDC lines are considered to be of concern as the limit increases with decreased frequency. With the high voltage and high current, it is important to keep the electromagnetic fields within control. For avoiding corona from the pole conductors, the bundle has a large diameter with many conductors. This minimizes the amount of corona from the DC line because the E-field around the pole conductors will be quite low. However, if not considering the E-field at the steel structures of the towers and the shield wires, the corona phenomena might occur on those structures instead, because of the much smaller radii of the earthed structures. The field levels for various structures may change with the mode of operation, i.e., bipolar or mono-polar operations. It must be noted that when the other line is left free floating in mono-polar operation, it will be charged to the same polarity as the pole line in operation, but with a lower voltage because of charging from the pole line in operation" (Lescale et al. 2008).

\section{Spares and maintenance}

"The spare inventory is directly related to the number of different types of equipment installed in the project and to the number of equipment installed. The most important equipment in the present context is the converter transformer and DC bypasses switches. Generally, when single-phase, two-winding transformers are employed, there are two types of converter transformers: one YY and another YD type. In case of two 12-pulse groups per pole because the voltage level of the lower 12-pulse group is substantially low compared to the upper group, one may opt to design different types of converter transformers for the two groups. This shall 
then require four types of spare converter transformers. The spares of converter bypass switches are required only when there are two 12-pulse groups per pole. Since there are 24 converter transformers in a case of two 12-pulse groups, compared to 12 converter transformers for one 12-pulse option, there shall be increased maintenance requirement in case of the former. The two-group option requires separate valve halls for the two groups so that maintenance can be carried out when one group is operating" (Huang et al. 2002).

Several advantages of DC systems over AC systems for integration of renewable energy are listed in Appendix 9.4.

\section{Feasibility of design and manufacturing of UHVDC equipment}

"The major concern on the feasibility of UHVDC up to $800 \mathrm{kV}$ is basically the oil-immersed equipment, such as converter transformer and oil-type smoothing reactor, while other DC equipment can be well designed to withstand the UHV stresses by scaling the design from existing 500-kV equipment supported by testing. This means that the UHVDC equipment will be considerably large in length and may consist of several units in series. The decisive measure for designing UHVDC equipment is to control the voltage distribution inside and outside of the equipment to avoid overstresses. It is a proven technology to control the DC voltage distribution by a predefined resistive current. Such technology can be found in the design of DC voltage divider, DC filter capacitor, DC PLC-coupling capacitor, and ZnO-arresters. The need to optimize the size and insulation level of major equipment would require careful selection and placement of $\mathrm{ZnO}$-arresters. This may call for the use of additional arresters at the UHVDC level, particularly for oil-filled equipment such as converter transformers. Another aspect, which is to be considered for UHVDC equipment, is the external insulation of outdoor equipment with considerations to environmental factors such as pollution, temperature, and height of installation. The creepage and clearance requirements may result in extreme dimensions in length, which may cause mechanical and manufacturing difficulties for equipment like disconnectors and wall bushings. It may be mentioned that because the flashover performance of outdoor insulators under polluted conditions depends to a large extent on the pollutant and atmospheric conditions (including orientation of installation because these may directly affect the non-uniformity of depositions and voltage distribution), an increase in specific creepage distance may not be the remedy under such conditions. Therefore, a reasonable external insulation level should be applied for such equipment. In case of need, other measures such as booster sheds may be applied to reduce the physical dimensions of the equipment. Maximum use of composite insulators is envisioned because it promises better performance and would enable use of smaller insulators. At present, it seems likely that some porcelain insulators will remain in the DC switchyard (e.g., mechanical strength considerations). Air core type smoothing reactors are likely the preferred solution for such UHVDC applications, particularly in the case of a configuration with two series groups per pole, or a distributed smoothing reactor concept with reactor coils in high voltage and neutral side. To meet the anticipated time schedule of the transmission projects, considerable investment in research and development in some key areas shall be conducted in advance to ensure the availability of design in time. Following areas may be included:

- Converter transformers;

- Bushings; and

- HVDC post-insulators/disconnector" (Huang et al. 2002).

An example of a HVDC project design can be also found in Huang et al. (2002). 
The national and international HVDC projects are presented in Appendix 9 (Section 5).

\subsubsection{Superconductor electricity pipelines ${ }^{24}$}

This section discusses superconductor pipeline design and the Tres Amigas project.

\subsubsection{Technology description, advantages and comparison with overhead AC lines}

"Superconductor electricity pipelines combine conventional underground pipeline techniques with two, highly complementary electric power options: superconductor cables and multiterminal (voltage-source converter-based) DC power transmission. The result is a high-capacity electric transmission "pipeline" that is underground, easy to access, highly efficient, controllable, and offers great security. This technology can control power flows to multiple destinations, across interconnections, and over long distances with minimal operating losses" (U.S. DOE 2010). Figure 4 shows a design of a superconducting pipeline (EPRI) reported in McCall (2009).

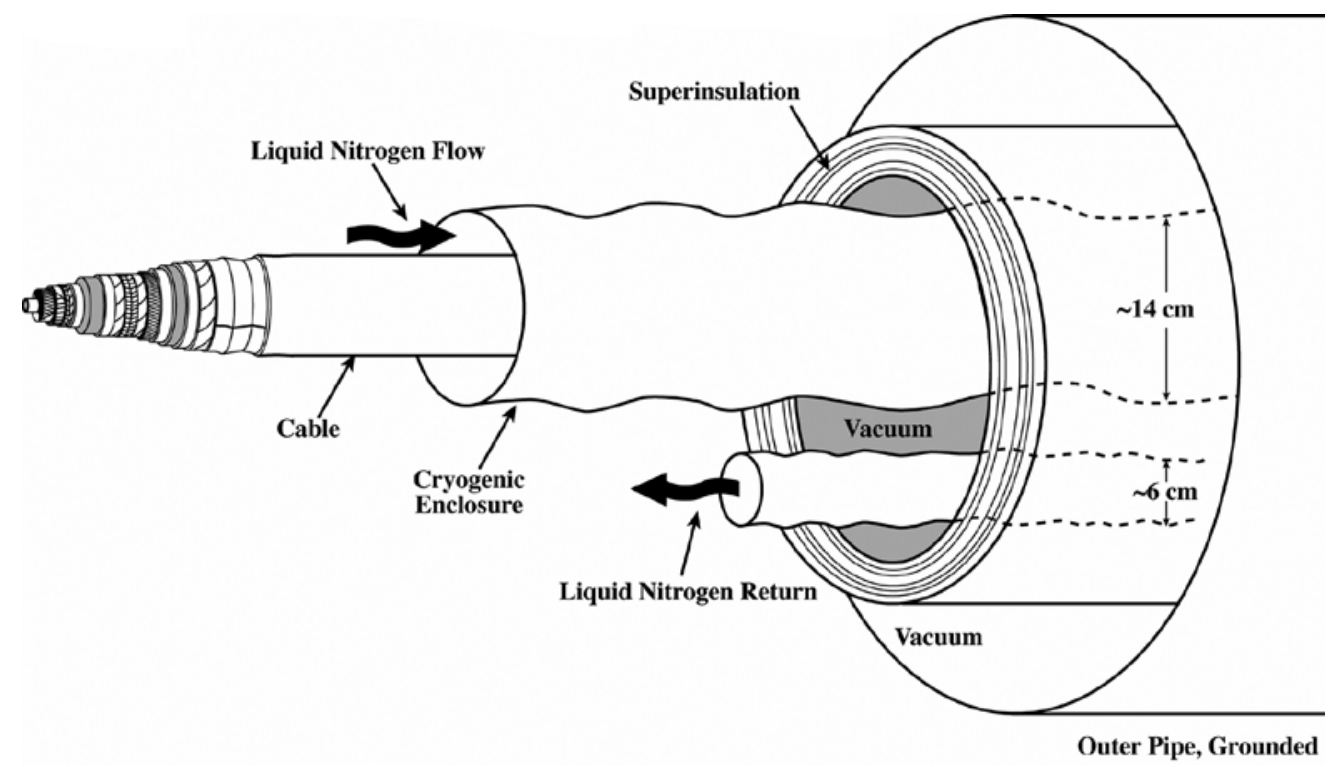

Figure 4. Superconductor pipeline design (Hassenzahl, W.V. Eckroad, S. E. C. Grant. P. M. Gregory, B. and Nilsson, S.2009) ${ }^{25}$.

In McCall (2009), the following advantages of superconductor pipelines are given:

- Underground construction with minimum right of way requirement;

- Simplified cost allocation due to precise controllability of DC terminals;

- High power capacity;

- Highest efficiency (lowest power losses) of any transmission technology;

${ }^{24}$ Contributed by Yuri Makarov, PNNL.

${ }^{25}$ Reused by permission of IEEE. 
- Work well for very long distances;

- Capable of transferring power across the three U.S. interconnections;

- Able to accept power from multiple distributed sources, and precisely deliver power to multiple distributed destinations; and

- Minimum interaction with existing $A C$ grid, reducing costs and increasing operational flexibility.

In Table 1, a comparison is made between the superconductor pipeline and an overhead 765$\mathrm{kV}$ transmission line designed to transfer $5 \mathrm{GW}$ over 1,000 miles (McCall 2009). The superconductor pipeline (unlike conventional AC lines) has power losses that do not depend on the distance (about 2-3 percent) - see Figure 5, and its transfer capability does not depend on line length and remains $5 \mathrm{GW}$ in the example (McCall 2009). These characteristics make the superconductor DC lines more attractive for long-length transmission systems and less attractive for short-length local transmission, because of the relatively high losses that may be less significant in short-length AC transmission lines.

Table 1. Comparison of DC superconductor cable with AC transmission line (McCall 2009). ${ }^{26}$

\begin{tabular}{|c|c|c|c|}
\hline & Metric & DC Superconductor Cable & $\begin{array}{c}\text { 765KV Transmission } \\
\text { Lines }\end{array}$ \\
\hline \multirow{6}{*}{ 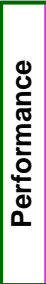 } & Power Loss ${ }^{(1)}$ & $3 \%$ & Varies with design \\
\hline & Storm/Security Risk & Low & High \\
\hline & Precise Control for Efficient Markets & Yes & No \\
\hline & Cost Allocation Method & Simple & Complicated \\
\hline & Requires Rebuild of Underlying Grid & No & Yes \\
\hline & "Black Start" Capability & Yes & No \\
\hline \multirow{4}{*}{ 点 } & Permanent Right of Way & $3 \mathrm{ft}$. & $400-600 \mathrm{ft}$. \\
\hline & Aesthetics & Good & Bad \\
\hline & Electromagnetic Field & None & High \\
\hline & New Land Required & No & Yes \\
\hline \multirow{3}{*}{ 范 } & Efficiency Savings Per Year(2) & $\$ 230$ million & $\mathrm{n} / \mathrm{a}$ \\
\hline & $\mathrm{CO}_{2}$ Emission Savings Per Year(2) & 3 million tons & $\mathrm{n} / \mathrm{a}$ \\
\hline & Cost Per Mile ${ }^{(3)}$ & $\begin{array}{l}\$ 8 \text { million for } 5 \text { GW pipe } \\
\$ 13 \text { million fully redundant }\end{array}$ & $\$ 9-10$ million minimum \\
\hline
\end{tabular}

\footnotetext{
${ }^{26}$ Reused by permission of IEEE.
} 


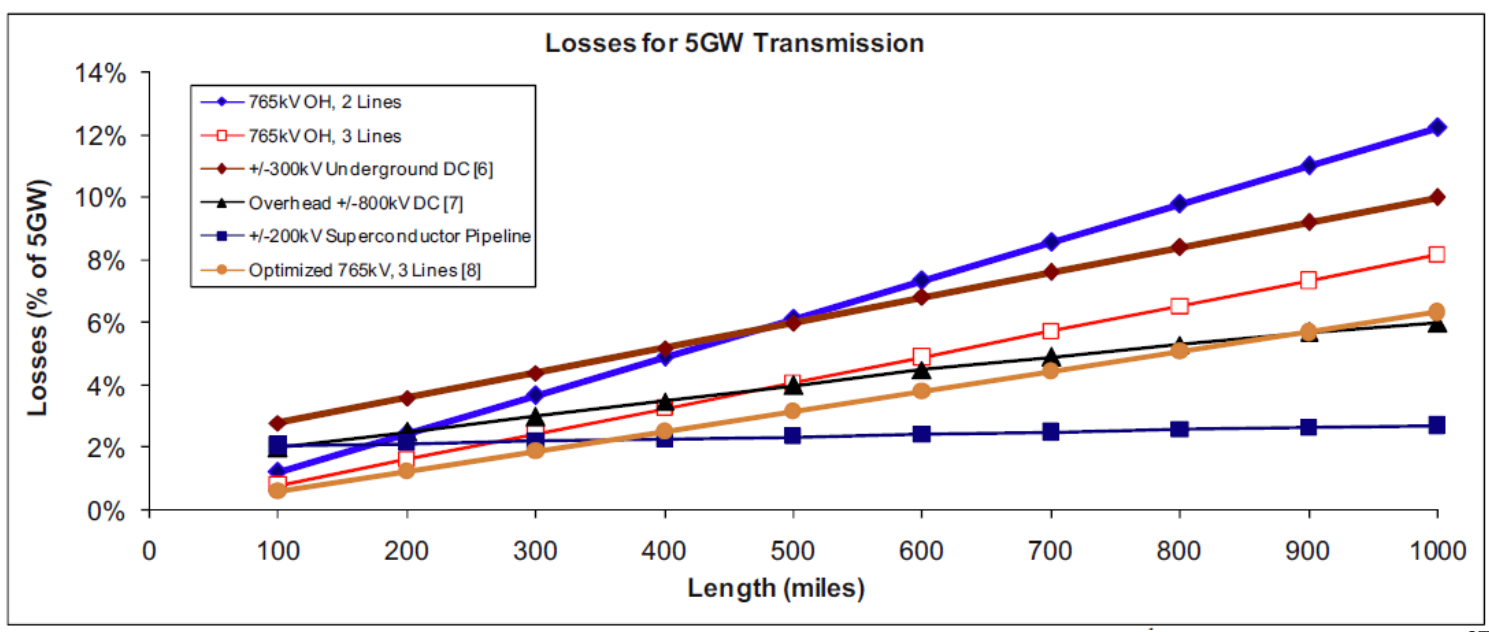

Figure 5. Losses for 5GW transmission (McCall, J. Gamble, B. and Eckroad, S. 2010). ${ }^{27}$

3.4.3.2 Tres Amigas project ${ }^{28}$ (TRESAMIGAS 2012) $^{29}$

The objectives of the proposed Tres Amigas project include:

- Connect the three U.S. synchronous power grids through a DC hub that can regulate the direction and level of power flows between the grids;

- Provide economic incentives to further drive the growth of the nation's renewable goals;

- Optimize the value of existing and planned AC infrastructure by utilizing state-of-the-art technology;

- Provide open access and cost effective transmission services consistent with NERC standards and regional reliability requirements; and

- Provide voltage support and capacity firming for renewables through larger generation portfolio across the regions.

The Tres Amigas superstation could start at $5 \mathrm{GW}$ and expand to $30 \mathrm{GW}$.

\subsubsection{Transmission upgrades}

"Due to the problems associated with constructing new overhead lines, it is important to examine the possible options for increasing the transmission capacity on present sites and making maximum use of existing transmission systems through upgrades. When feasible, upgrades are an attractive alternative, because the costs and lead times are less than those for constructing new lines" (Larruskain et al. 2007).

\footnotetext{
${ }^{27}$ Reused by permission of IEEE.

${ }^{28}$ Contributed by Yuri Makarov, PNNL.

${ }^{29}$ Contributed by Yuri Makarov, PNNL.
} 


\subsubsection{Replacing limiting elements}

In many cases, the transmission lines' transmission capacity is limited by certain elements, e.g., switchgear elements (Larruskain et al. 2007), jumpers, and certain line sections. Replacing or upgrading those elements helps improve overall transmission capacity.

\subsubsection{Voltage uprating ${ }^{30}$ (Holman 2011)}

"Voltage uprating is used to increase a transmission line voltage to the next standard level. Thus, the power that can be transmitted through the transmission line is increased. Some older transmission lines have been overbuilt to the extent that their voltage can be increased with limited changes to the transmission line itself. An increase in the corona of the transmission line and its electromagnetic field may still require an expansion of the right-of-way (ROW). If those challenges can be overcome, voltage uprating can be a good option for increasing the capacity of a transmission line. In some cases, voltage uprating requires a larger conductor or bundled conductors, and larger transformers. Then, it requires installation of new conductors and new towers, or modification of existing towers. One advantage of rebuilding the transmission line in this way is that it allows using a compact design or bundling the conductors so that the corona and electromagnetic field do not need an expanded ROW. This reduces the impact of the upgrade on the footprint of the transmission line, and could make planning and permitting easier while reducing costs" (Holman 2011).

"During the last years, there have been many efforts to make appropriate modifications to existing overhead lines, and to eliminate the old $A C$ transmission lines and substitute them with new compact AC lines. Both of these solutions lead to an increase in the transmitted power by the overhead line, increasing the rated voltage. This is possible by utilizing the experience acquired for HVAC lines and by permitting reduced safety margins in designing clearances. For compact AC lines, insulated crossarms and a shorter span are also used, thereby reducing the line sag so that a substantial increase in the power density is achieved" (Larruskain et al. 2007).

\subsubsection{Series compensation and reactors}

"Voltage on a transmission line tends to "drop" from the sending end to the receiving end. The voltage drop along the AC line is almost directly proportional to reactive power flows and line reactance. The line reactance increases with the length of the line. Capacitors and inductive reactors are installed, as needed, on lines to, partially, control the amount of voltage drop. This is important because voltage levels and current levels determine the power that can be delivered to the customers" (Larruskain et al. 2007).

"The simplest form of series compensation is known as fixed series compensation (FSC), the main benefit of which is to allow an increase in transmission capacity. The more sophisticated thyristor controlled series capacitor (TCSC) is deployed if fast control of the line impedance is required for load-flow control and for damping power oscillations. It also has the benefit of enabling the mitigation of sub-synchronous resonances" (Alberta Energy 2009).

"Figure 6 shows a simplified single line diagram of an FSC - three such circuits are used, one per phase of the transmission line" (Alberta Energy 2009).

${ }^{30}$ Tres Amigas: http://www.tresamigasllc.com/. 


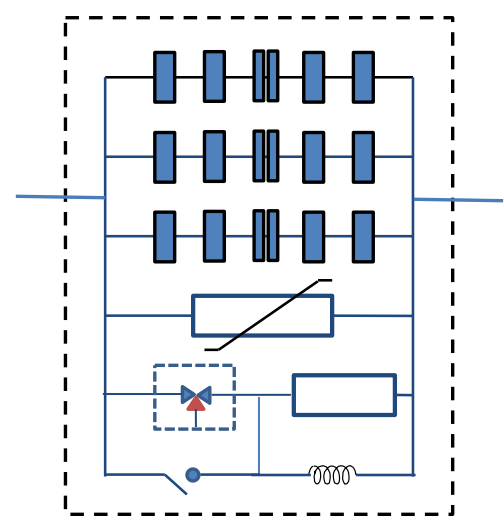

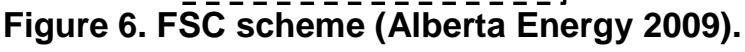

"Each capacitor bank within the FSC would be made up of many capacitors connected in a series/parallel arrangement. Also shown is the MOV (metal oxide varistor), triggered spark gap and damping circuit, which forms the bypass system to prevent damage to the capacitor in the event of an overvoltage surge. Also shown is the bypass switch. It is turned on when line faults cause high currents, which would otherwise cause high voltages across the capacitor bank. The total value of the capacitance and the number of capacitors installed in the capacitor bank is determined by the compensation requirements calculated as a result of the essential network system study, which must be executed before a specification can be created" (Alberta Energy 2009). Graphical representation of a typical FSC installation, showing all three phases built on insulated platforms can be found in (Alberta Energy 2009).

An additional advantage of series compensation is that, once installed, it breaks circuits for slowly changing geomagnetically induced currents (GIC), which are caused by solar storms. Those currents can saturate power transformers and could cause damage from overheating.

"The FSC has a long history with the first systems being put into service during the mid-1950s (since the 1960s for 500-kV versions); and approximately 610 FSC systems in total are in operation today around the world with ratings from 10 Mvar to 1,500 Mvar, with typical ratings of 150 Mvar to 300 Mvar" (Alberta Energy 2009).

\subsubsection{Increasing current density}

"When the flow of electrons goes through the line, it produces heat and the conductor's temperature increases. It is necessary to make a thermal study to know if the conductor can stand that temperature. The temperature of the conductor is limited by two factors:

1. The limit of the conductors material; and

2. Conductor - ground distance" (Larruskain et al. 2007).

"There are different ways to achieve this increase:

1. Increase the maximum allowable operating temperature to $100^{\circ} \mathrm{C}$. For example, if the line is limited to a modest temperature of $50^{\circ} \mathrm{C}$ to $75^{\circ} \mathrm{C}$, and the electrical clearance is sufficient to allow an increase in sag for operation at a higher temperature, then the thermal rating of the line can be increased. If sufficient clearance does not exist in all spans, then conductor attachment points may be raised, conductor tension increased or 
other mechanical methods applied to obtain the necessary clearance at the higher temperature.

2. Use dynamic ratings or less-conservative weather conditions relating to wind speed and ambient temperatures. For example, if the existing line is already rated at a temperature near $100^{\circ} \mathrm{C}$, and a modest increase of 5 percent to 15 percent is desired, then monitors can be installed and the higher ratings can be used when wind speed is higher than the standard $0.6 \mathrm{~m} / \mathrm{s}$, and the ambient temperature is lower than $40^{\circ} \mathrm{C}$.

3. Replace the conductor with a larger one, or with one capable of continuous operation above $100^{\circ} \mathrm{C}$. These solutions would be ideal if the line was already limited to $100^{\circ} \mathrm{C}$, and the thermal rating increased by more than 25 percent. Given the low cost, high conductivity, and low density of aluminum, no other high-conductivity material is presently used. Therefore, replacement with a larger conductor will result in an increased load on existing structures because of an increase of wind/ice and tension" (Larruskain et al. 2007).

\subsubsection{Rewiring}

The concept of rewiring is to "replace the conductor with a larger one or with a one capable of continuous operation above $100^{\circ} \mathrm{C}$. These solutions would be ideal if the line was already limited to $100^{\circ} \mathrm{C}$, and the thermal rating increased by more than 25 percent. Given the low cost, high conductivity and low density of aluminum, no other high-conductivity material is presently used. Therefore, replacement with a larger conductor will result in an increased load on existing structures because of an increase of wind/ice and tension" (Larruskain et al. 2007) Additional information on this is presented in Appendix 10.

\subsubsection{High-temperature low-sag (HTLS) conductor}

"The majority of overhead transmission lines use steel-reinforced aluminum conductors (ACSR). On a continuous basis, ACSR may be operated at temperatures up to $100^{\circ} \mathrm{C}$ and, for limitedtime emergencies, at temperatures as high as $125^{\circ} \mathrm{C}$ without any significant change in the conductor's physical properties. Replacing original ACSR conductors with high-temperature, low-sag (HTLS) conductors with approximately the same diameter is one method of increasing transmission line thermal rating. HTLS conductors are effective because they are capable of (1) high-temperature, continuous operation above $100^{\circ} \mathrm{C}$, without loss of tensile strength or permanent sag-increase (so that line current can be increased), and (2) low sag at high temperature (so that ground and underbuild clearances can still be met without raising or rebuilding structures)" (Douglas 2002).

"Conductors are constructed from helically stranded combinations of individual wires where galvanized steel wires are used for mechanical reinforcement, aluminum wires for the conduction of electricity, and hard-drawn aluminum for both mechanical and electrical purposes.

Desirable properties for reinforcing core-wire material include a high elastic modulus, a high ratio of tensile strength to weight, the retention of tensile strength at high temperatures, a low plastic and thermal elongation, a low corrosion rate in the presence of aluminum and a relatively high electrical conductivity. The material must be easy to fabricate into wire for stranding" (Larruskain et al. 2007).

Although HTLS conductors allow reusing the same ROW and towers, they are significantly more expensive than traditional cables. In some situations, such as long spans over rivers that are 
particularly sensitive to sag, HTLS conductors can play an important role. They also can improve power grid reliability without installing additional transmission lines. Thus, the additional capacity enabled by the HTLS cable's ability to carry high currents allows other transmission lines in the area to increase their capacity. If one of those other transmission lines fails, the HTLS cable can carry the additional load until the down line is repaired (Holman 2011).

Different types of HTLS conductors are listed in Appendix 11.

\subsubsection{AC/DC conversion (Larruskain et al. 2007)}

"The AC line can be converted to three DC circuits, each having two conductors at $\pm V_{d}$ ( $V_{d}$ is DC pole to earth voltage) to earth respectively. On the basis of equal current and insulation, the power transmitted by overhead lines can be increased to 147 percent, with the percentage line losses reduced to 68 percent, and corresponding figures for cables are 294 percent and 34 percent, respectively. Besides, if the AC line is converted, a more substantial power upgrading is possible. There are several proposals to convert AC lines to DC lines, and these conversions are carried out as a simple reconstruction. The most feasible of them is double circuit AC conversion to bipolar DC; it implies tower modifications that maintain all the conductors at a height above ground of 1 to 2 meters below the original position of the lowest conductor during the whole construction phase. Two new crossarms are inserted at the level of the old intermediate crossarm. No change is made to the conductors, the total rated current remains the same, which means that the transmitted power increases proportionally to the adopted new DC line-to-ground voltage. The conversion of lines, where an increase of phase to ground voltage can be higher than three, is possible when all the conductors of one AC circuit are concentrated in one DC pole. The line-to-line AC voltage is doubled for use with DC, thus the transmitted power will increase by 3.5 times" (Larruskain et al. 2007).

\subsection{Grid Stability ${ }^{31}$}

Stability can be defined as an electrical power system's ability to return to a steady state after a disturbance. Power system stability analysis focuses on online synchronous machine behavior after a system disturbance with two possible outcomes after any disturbance: (1) the machines return to their original state (the system demonstrates post-disturbance positive damping and no net change in power occurs), all the synchronous machines remain in synchronism (operating at the same speed), and/or the system is stable (as demonstrated by relative rotor angles); or (2) the machines enter a new operating state (imbalance between the generation and load occurs) (Kravchuk and Pierce 2009). In the latter state, it is possible for uncontrollable grid oscillations to cause generator damage, other equipment damage, and/or other system operating condition problems that electrical system operators must address on a continual basis. The existence and increase of variable generation has an effect on the system stability considerations that must be made now and in the future. Figure 7 gives the overall picture of the power system stability problem:

\footnotetext{
${ }^{31}$ Contributed by David Tovar, EPE, Jian Ma, PNNL, Pengwei Du, PNNL, and Yuri Makarov, PNNL.
} 


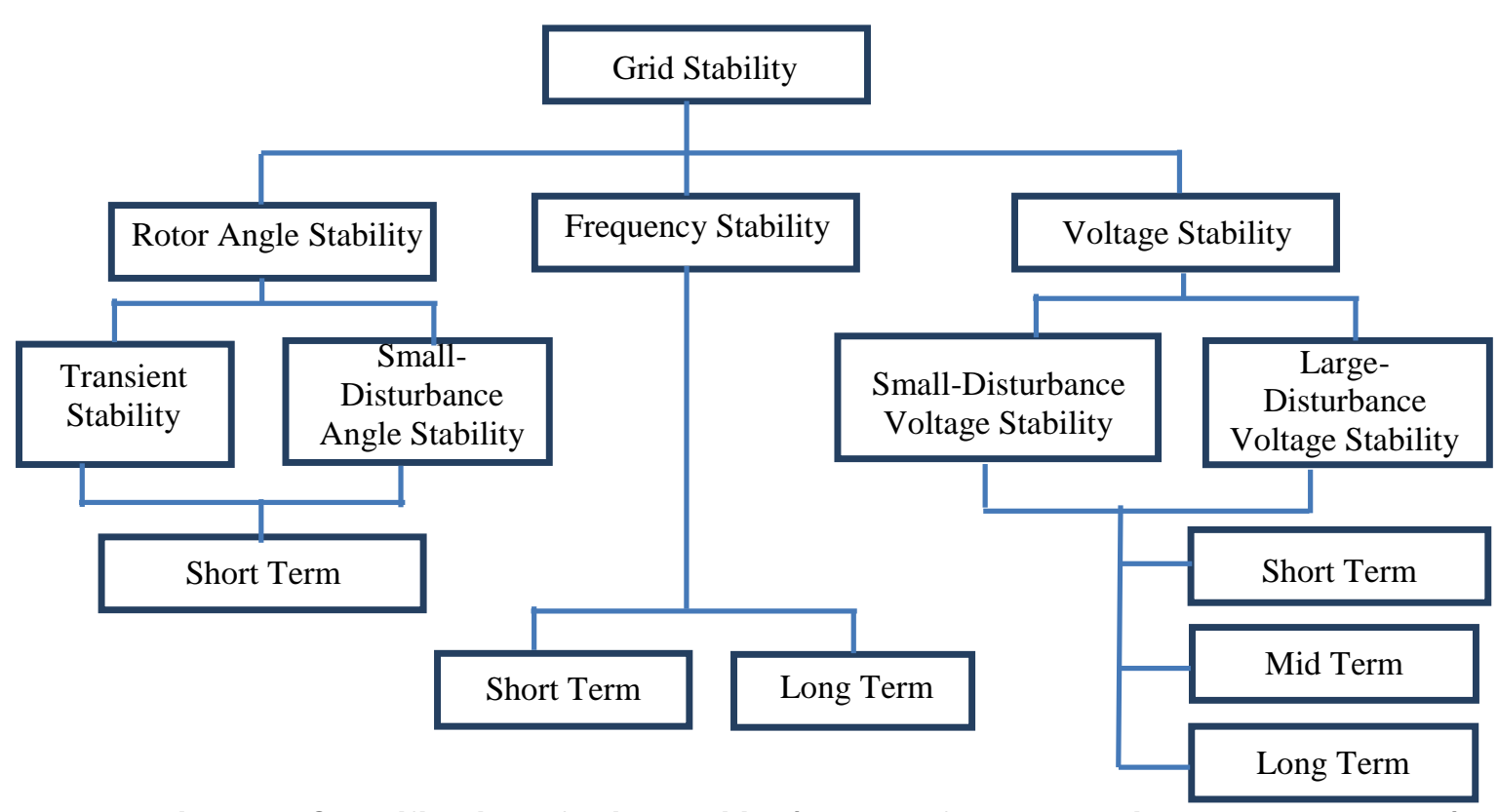

Figure 7. Classification of grid stability (adapted from Kothari and Nagarath 2011).

The effects of stability can be classified into: (1) small-signal effects; and (2) large-signal effects. There are different types of stability:

1. Rotor angle stability is the ability of synchronous machines interconnected through an electrical power system to remain in synchronism (e.g., in the U.S. at $60 \mathrm{~Hz}$ ) after a small disturbance on that system, called small-signal stability, or after a large disturbance on that system, called large-signal or transient stability. As such, rotor angle stability deals with generator stability and real power. A disturbance can cause: 1) angular swings in some generators caused by differences in electromagnetic and mechanical torque (these torques are equal in equilibrium or prior to a disturbance); 2) rotor acceleration or deceleration; and possibly 3 ) a slip in field synchronization causing a generator or generators to fall out of synchronism with the rest of the electrical system - loss of generator synchronism disconnects it from the electrical system (Bragason 2005). In equilibrium, the speed of the rotor and the angle between the stator and rotor magnetic fields are constant (Bragason 2005). Rotor angle instability occurs with the loss of synchronism of one or more synchronous generators usually after a disturbance (Reactive Reserve Working Group 2006).

2. Frequency stability is defined as an electrical power system's ability to maintain system frequency in a required range and at an acceptable level, after a disturbance or disturbances that causes a load and generation imbalance. Frequency instability occurs when system load and losses do not match online generation to serve that load and losses - usually after a major electrical system disturbance. With frequency instability, loss of load and/or losses can occur within an electrical system. Frequency stability can be grouped into two main time frames of interest: 1) short-term phenomenon; and 2) long-term phenomenon.

3. Voltage stability is defined as an electrical power system's ability to maintain voltages within a range of required and/or acceptable levels at all buses under normal (no disturbance) conditions; or after a disturbance or disturbances in the transient, post- 
transient, and long-term time frames. Voltage stability deals with load stability and reactive power after a disturbance. Voltage instability can occur when there is a loss of a stable operating point at a bus or buses - as characterized by a violation of some voltage criteria. This may include operating outside required or acceptable voltage ranges, which characterize voltage stable operation at that bus and buses. Rotor angle stability and voltage stability are related because rotor angle swings can cause voltage swings. Rotor angle instability (perhaps caused by a major disturbance) can result in voltage instability.

Voltage stability can be grouped into three main time frames of interest: 1 ) short-term, time between disturbance and automatic transformer load-tap-changing (LTC) action; 2) mid-term, time after disturbance and after LTC action and prior to over-excitation limiter (OEL) action; and 3) long-term, time after disturbance and after OEL action that includes manual system operator mitigating actions (Reactive Reserve Working Group 2006).

Selection of stability criteria depends on the type of stability problem being studied. Typical criteria for the most common security problems are given below: (Makarov et al. 2009)

- Voltage instability: Mvaror MW "distance" to the nearest instability point, e.g., the distance to the "nose" point of a Q-V or P-V curve or distance to the closest instability point in the multidimensional space of system parameters, such as nodal injections or power flows on selected transmission paths.

- Transient or dynamic instability: transient voltage dip, transient frequency dip, energy margin, or critical fault clearing time.

- Oscillatory or small signal instability: damping ratio or the real part of a dominant eigenvalue, corresponding to a potentially unstable mode of oscillations.

\subsubsection{Dynamic or transient stability ${ }^{32}$}

Transient, or first-swing, stability deals with a power system's ability to maintain synchronism after a large signal or severe transient disturbance (e.g., a fault, loss of generation, line switching, line outage(s), and/or loss of a large load). Transient stability studies are performed using software that performs time-domain simulations with databases that include models for generators, excitation systems, prime-mover/turbine/turbine governor, load characteristics (e.g., percent motor load), and other electrical devices that influence electrical system transient stability (e.g., power system stabilizers, under-voltage relays, under-frequency relays). Transient stability studies, usually examining the time from 0 seconds up to 10 seconds or more (usually not more than 20 seconds) after a disturbance, are performed to make a determination of whether or not an electrical system under study will remain in synchronism (maintaining the balance between the online generator-produced electric power and the load-consumed power). Minimum performance criteria after a disturbance for each stability type - rotor angle, voltage and frequency - can be applied to transient stability study results. Several different disturbances are studied and any violations of minimum performance are reported. As such, transient stability studies can be used to make an evaluation of the ability of electric generators online in an electrical system to recover from a disturbance. They also evaluate how they can remain connected to the system following a disturbance, and/or how these generators interact with one another with regard to rotor angle stability and with regard to voltage and frequency stability.

\footnotetext{
${ }^{32}$ Contributed by David Tovar, EPE, Pengwei Du, PNNL, and Yuri Makarov, PNNL.
} 
There are three main approaches to the transient stability assessment problem:

1. The classical method of the transient stability analysis is carried out through a series of system time-domain simulations in a broad range of situations. Transient stability criteria are applied to distinguish stable system trajectories from unstable ones. One of the advantages of the time-domain simulation method is its precision and unlimited modeling capability. Despite the advantages, the time-domain simulation method has two main shortcomings. First, it is inherently slow because of integration process involved in solving the differential-algebraic equations. Second, it does not yield the information on the system's degree of stability if it is found stable.

2. The energy functions method and the equal area criteria are exploited to estimate the region of stability or attraction area in coordinates of power system parameters. They can directly provide stability information without solving differential equations. However, they have modeling restrictions and address only the first swing after a disturbance. The hybrid method combines the time domain and the energy function methods. It first computes the system trajectory using time-domain simulation, then it calculates the degree of stability by evaluating the transient energy function.

3. Artificial intelligence (AI) methods can be used as an approach for transient stability assessment. This approach decomposes transient stability problems into elementary problems. The elementary transient stability assessment problems are defined for a given network topology and contingency. For each elementary problem, the Al method builds a set of decision rules offline using a large number of simulations ("learning set"). Online assessment employs the decision rules for the given operating condition to evaluate transient stability for the analyzed cases.

\subsubsection{WECC transient stability analysis using classical method}

In the WECC system, transient stability is evaluated using the WECC reliability criteria (WECC 2008), the WECC voltage stability criteria, and WECC members' reliability criteria as appropriate. Accordingly, a Category B disturbance in one system shall not cause a transient voltage dip in another system that is greater than 20 percent for more than 20 cycles at load buses, or exceed 25 percent at load buses or 30 percent at non-load buses at any time other than during the fault. This disturbance also can not cause the frequency to drop below $59.6 \mathrm{~Hz}$ for six cycles or more at a load bus. This disturbance standard, which all WECC member systems must comply with, is listed in and illustrated in Table 2 and Figure 8.

Transient stability simulations (TSS) 100-MW stability margin test is applied to each stability boundary point (positive damping at power margin $100 \mathrm{MW}$ ).

"NERC/WECC Planning Standards I.D also addresses the risk of widespread voltage collapse by establishing the need for sufficient static and dynamic reactive power support within the power system under consideration. There are five standards under this category, four of which are specific to WECC member systems (Standards WECC-S1 through WECC-S4) (WECC 2006).

The standard common to all NERC Regional Reliability Councils (RRC) is as follows:

S1. Reactive power resources, with a balance between static and dynamic characteristics, shall be planned and distributed throughout the interconnected transmission systems to ensure system performance as defined in Categories $A, B$, and C of Table I in the I.A. Standards on transmission systems" (WECC 2006).

Four WECC-specific standards are as follows: 
WECC-S1. For transfer paths, post-transient voltage stability is required with the path modeled at a minimum of 105 percent of the path rating (or operational transfer capability) for system normal conditions (Category A) and for single contingencies (Category B). For multiple contingencies (Category C), post-transient voltage stability is required with the path modeled at a minimum of 102.5 percent of the path rating (or operational transfer capability).

WECC-S2. For load areas, post-transient voltage stability is required for the area modeled at a minimum of 105 percent of the reference load level for system normal conditions (Category A) and for single contingencies (Category B). For multiple contingencies (Category $\mathrm{C}$ ), post-transient voltage stability is required with the area modeled at a minimum of 102.5 percent of the reference load level. For this standard, the reference load level is the maximum established planned load limit for the area under study.

WECC-S3. Specific requirements that exceed the minimums specified in I.D WECC-S1 and S2 may be established, to be adhered to by others, provided that technical justification has been approved by the WECC Planning Coordination Committee.

WECC-S4. These standards apply to internal WECC member systems as well as between WECC member systems (WECC 2006). 
Table 2. WECC disturbance-performance table of allowable effects on other systems (WECC 2008).

\begin{tabular}{|c|c|c|c|c|}
\hline $\begin{array}{l}\text { NERC and } \\
\text { WECC } \\
\text { Categories }\end{array}$ & $\begin{array}{l}\text { Outage Frequency } \\
\text { Associated with the } \\
\text { Performance } \\
\text { Category } \\
\text { (outage/year) }\end{array}$ & $\begin{array}{l}\text { Transient Voltage } \\
\text { Dip Standard }\end{array}$ & $\begin{array}{l}\text { Minimum } \\
\text { Transient } \\
\text { Frequency } \\
\text { Standard }\end{array}$ & $\begin{array}{l}\text { Post Transient } \\
\text { Voltage } \\
\text { Deviation } \\
\text { Standard }\end{array}$ \\
\hline A & Not Applicable & $\begin{array}{l}\text { Nothing in addition to } \\
\text { NERC }\end{array}$ & & \\
\hline B & $\geq 0.33$ & $\begin{array}{l}\text { Not to exceed } \\
25 \% \text { at load buses } \\
\text { or } 30 \% \text { at non- } \\
\text { load buses. Not to } \\
\text { exceed } 20 \% \text { for } \\
\text { more than } 20 \\
\text { cycles at load } \\
\text { buses. }\end{array}$ & $\begin{array}{l}\text { Not below } 59.6 \\
\mathrm{~Hz} \text { for six cycles } \\
\text { or more at a load } \\
\text { bus. }\end{array}$ & $\begin{array}{l}\text { Not to exceed } \\
5 \% \text { at any bus. }\end{array}$ \\
\hline C & $0.033-0.33$ & $\begin{array}{l}\text { Not to exceed } \\
30 \% \text { at any bus. } \\
\text { Not to exceed } \\
20 \% \text { for more than } \\
40 \text { cycles at load } \\
\text { buses. }\end{array}$ & $\begin{array}{l}\text { Not below } 59.0 \\
\text { Hz for six cycles } \\
\text { or more at a load } \\
\text { bus. }\end{array}$ & $\begin{array}{l}\text { Not to exceed } \\
10 \% \text { at any bus. }\end{array}$ \\
\hline D & $<0.033$ & $\begin{array}{l}\text { Nothing in addition to } \\
\text { NERC }\end{array}$ & & \\
\hline
\end{tabular}

VOLTAGE PERFORMANCE PARAMETERS

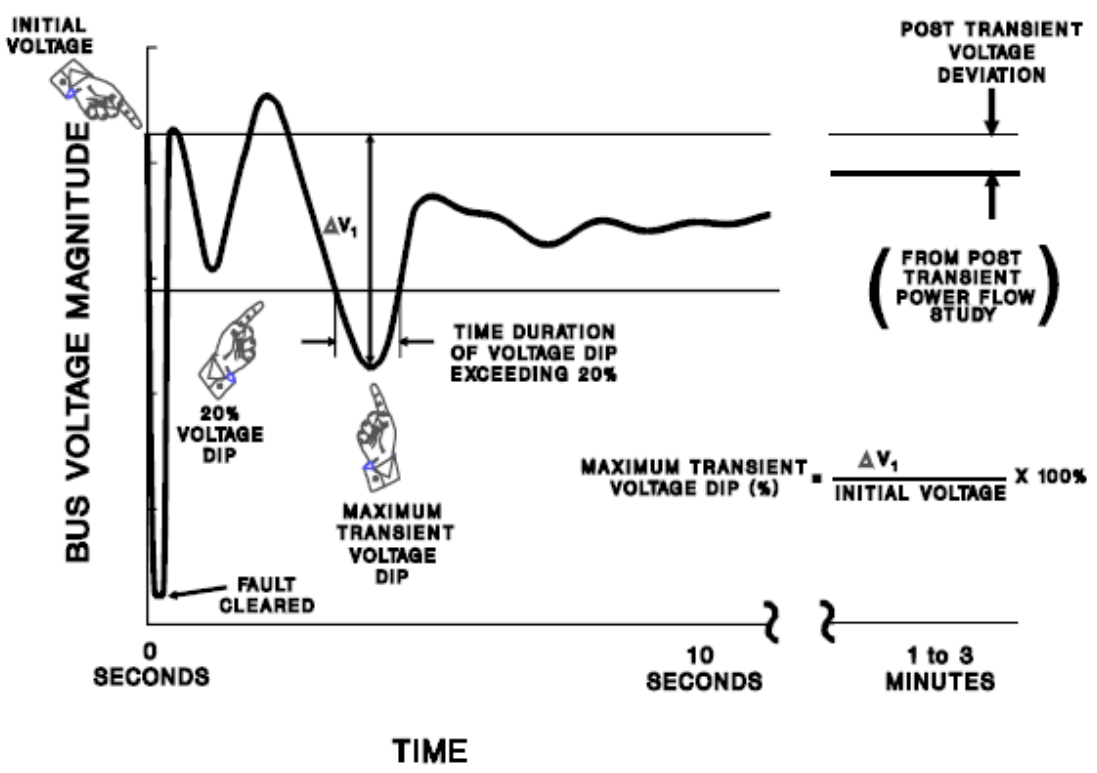

Figure 8. Voltage transient performance parameters (WECC 2008).

Direct method and energy functions are presented in Appendix 12. 
3.5.1.2 Stability margin and sensitivities analysis (Tong et al. 1993, El-Kady et al.1986) ${ }^{33}$ The transient stability margin is given as

$$
\varepsilon=V_{C R}-V_{c l}
$$

where $V_{C R}$ and $V_{c l}$ denote the system critical energy and transient energy at the fault clearing moment. Direct methods open possibilities toward online analytic sensitivity analysis and preventative control. Numerical methods have been developed to use the sensitivity of the energy margin $\Delta \varepsilon$ to changes in system parameters to determine system stability limits. In ElKady et al. (1986), an approach to finding the sensitivity of $\Delta V$, using a iterative analysis through the transient energy function method and linearized margin assumption, was used in the computation of interface low limits with power flow distribution factors. A theoretical foundation has been described in Tong et al. (1993) to decide how to make changes in the parameters, through action such as generation rescheduling and load shedding, to improve the system's transient behavior. It is found that for small changes in generation, the first-order sensitivity technique quickly identifies the new system's stability energy margin with a high degree of accuracy.

\subsubsection{Transient stability and variable generation ${ }^{34}$}

With the increasing integration of wind power, wind turbines are required to ride through the temporary faults. This has resulted in greater emphasis on transient stability performance, power oscillations and system damping (Rueda and Shewarega 2009). Recent research has revealed that transient stability of the system is enhanced when variable speed wind turbines, using doubly-fed induction generators (DFIG), are integrated into the system instead of fixedspeed turbines. But a drawback of using grid-connected, power electronic converter-based DFIGs is their sensitivity to grid disturbances (Vittal et al. 2009).

\subsubsection{Voltage stability ${ }^{35}$}

The content of this section in a large extent follows references Parashar et al. (2008) and Parashar et al. (2007a).

\subsubsection{Description of the voltage stability problem ${ }^{36}$}

An electrical power system is voltage stable if acceptable voltages (that meet specific minimum performance criteria) are maintained at all buses in the system under normal operating conditions, and after being subjected to a disturbance (Parashar et al. 2007b). "Regulating mechanisms try to restore power to meet demand at normal voltage. Load restoration further stresses and overloads power systems, resulting in voltage instability and collapse - voltage stability is load stability, related to load demand versus load capability" (Taylor 2008). A system is voltage unstable if, for at least one system bus, the voltage magnitude decreases as reactive power injection is increased. Voltage instability results in the progressive fall or rise of voltages of some buses.

Voltage stability is maintained by ensuring that reactive power demand and supply balance is maintained in the system at all times. Reactive power consumption by electrical loads drives voltage instability/load instability. Other factors influencing this phenomenon are the strength of

\footnotetext{
${ }^{33}$ Contributed by Pengwei Du, PNNL.

${ }^{34}$ Contributed by David Tovar, EPE.

${ }^{35}$ Contributed by Yuri Makarov, PNNL.

${ }^{36}$ Contributed by David Tovar, EPE, and Yuri Makarov, PNNL.
} 
the transmission system, generator reactive power, voltage control limits, characteristics of reactive compensating devices, and the action of the under-load tap changer (ULTC) under lowvoltage conditions (Rahmatullah 2006). Reactive power cannot travel long distances, and therefore reactive power demand has to be supplied by reactive power sources distributed around the network such as capacitor, Static VAr compensator (SVC), generator, and line and cable shunt capacitors.

"A system enters a state of voltage instability when a disturbance, increase in load demand, or change in system condition causes a progressive and uncontrollable decline in voltage. The main factor causing voltage instability is the inability of the power system to meet the demand for reactive power. Voltage stability is the ability of a power system to maintain acceptable voltages at all buses in the system under normal operating conditions and after being subjected to a disturbance. Voltage stability margin is the distance to instability determined for a selected loading or stress direction in parameter space. It is known that voltage magnitudes alone are poor indicators of voltage stability or security. Voltages can be near normal with generators, synchronous condensers, and SVCs near current limiting levels, thus resulting in a possible voltage collapse. Therefore, it is prudent to specify a Mvar margin or MW margin" (Sauer et al. 2004).

Venikov and co-authors (1975) first demonstrated that under certain model assumptions, such as constant voltages of the generator buses, matching load models and the same slack bus model, the static aperiodic stability limit or the saddle node bifurcation coincides with the power flow feasibility limit. This allows analyzing aperiodic stability limits using conventional power flow models under these modeling assumptions. Generally speaking, for the voltage stability problem, the assumptions do not hold. But nevertheless, the power flow model, and the power flow Jacobian matrix singularities are frequently used for an approximate description of the static aperiodic voltage stability problem. This reference (Kundur, 1994) provides some practical applications of this methodology.

Many system blackouts were related to voltage instability and voltage collapse problems. Some of those events and WECC's summary of recommendations are listed in Appendix 13.

\subsubsection{WECC Voltage Stability Criteria ${ }^{37}$ (WECC 2006)}

WECC's voltage stability criteria are specified in WECC (2006). Planning Standard S1 states that reactive power resources in an area should consist of a "balance" between static and dynamic characteristics. Dynamic reactive power resources refer to equipment that can respond within cycles of a disturbance, for example, to avert the risk of voltage collapse in instances where static shunt devices are not capable of reacting fast enough. The recommended method of identifying the appropriate balance between static and dynamic reactive power reserves is to undertake time-domain or dynamic simulations. Alternatively, an approximated method of determining the appropriate balance may be harnessed from the development of the $\mathrm{V}-\mathrm{Q}$ curves, as proposed by Conseil International des Grands Réseaux Électriques (CIGRE) (French: International Council for Large Electric Systems; founded in 1921) and incorporated in the aforementioned WECC publication.

\footnotetext{
${ }^{37}$ Contributed by Yuri Makarov, PNNL
} 
The minimum required amount of dynamic reactive power resource to obtain a feasible operating point is equal to the reactive-power margin deficit (difference between $x$-axis and the curve minimum), depicted by the following $\mathrm{V}$ - $\mathrm{Q}$ curve of a reactive deficient system:

A curve, representing a base loaded or base interface flow case, increased by 5 percent (per WECC-S1 or WECC-S2) incorporating: a) voltage-dependent static load models, b) worst category B disturbance, and c) no LTC operation.

In the curve, as shown in Figure 9, the model represents a simulation of the system (if the load or transfer were increased by 5 percent) in the short-term timeframe, incorporating voltage dependent load characteristics.

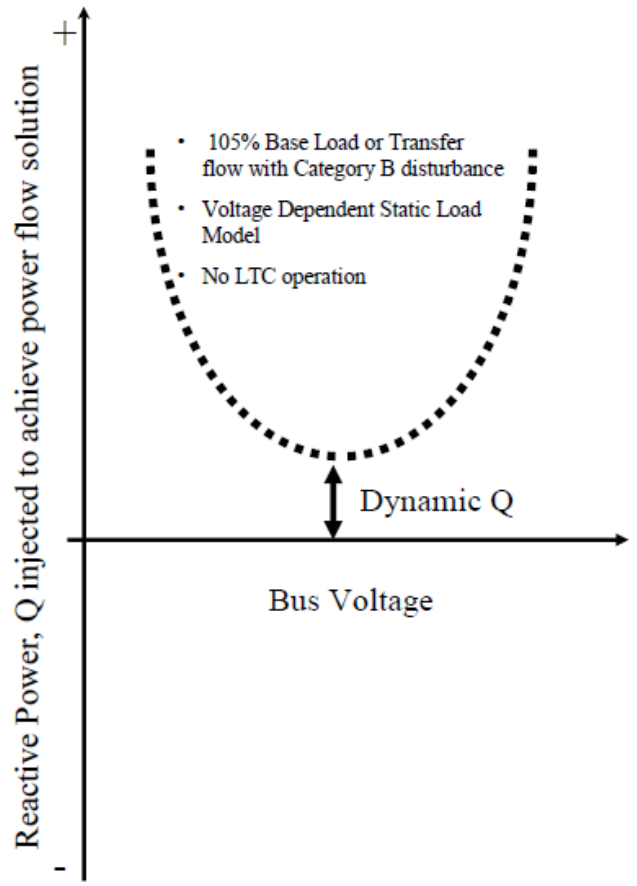

Figure 9. Determination of the dynamic reactive power resource requirement (WEEC 2006).

In the mid-term to long-term time frame, automatic load tap changers (LTC) restore voltages at load buses, resulting in loads behaving with or approaching constant power characteristics. Constant impedance type loads, providing load relief in the short-term time frame, begin to draw more current as voltage is increased over the mid-term and long-term time frame. During this period, static reactive power resources can be deployed to provide support. The amount of static reactive power resource required within a reactive-power deficit area is determined as the difference between the minima of the following two $\mathrm{V}-\mathrm{Q}$ curves of a reactive deficient system (refer to Figure 10):

A curve representing a base loaded or base interface flow case increased by 5 percent (per WECC-S1 or WECC-S2) incorporating: a) constant power load models, b) worst category B disturbance, and c) automatic LTC operation.

The V-Q curve of Figure 9. 
Therefore, the total minimum amount of reactive power resources that is required to obtain a feasible operating point in a reactive power deficit area, is equal to the sum of the dynamic and static reactive power resources, as calculated above.

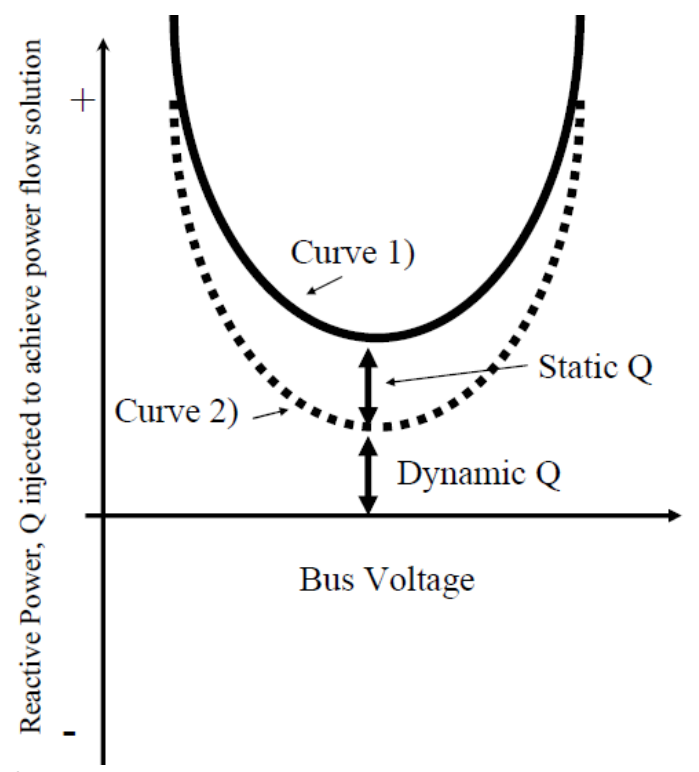

Figure 10. Determination of the dynamic reactive power resource requirement (WECC 2006).

\subsubsection{Definitions ${ }^{38}$}

Voltage collapse is the process or sequence of events accompanying voltage instability that leads to unacceptable voltage profiles in a significant part of the system (Kundur 1994). Many voltage collapses are not concentrated in a few power system buses. Rather, the collapse is regional or involves the entire system. Utilities spend considerable resources assembling sets of patterns of loading that the power system could experience in the next few hours. The ability of voltage security assessment (VSA) to predict voltage profiles will benefit utilities by pruning the worst-case patterns of loading.

The power flow problem is described by a set of nonlinear algebraic equations that are generally different, compared to the set of nonlinear algebraic equations obtained from the differential equations and that describe the static voltage stability phenomena. The power flow calculations normally use simplified component models and involve secondary control models such as generation dispatch, multi-area power flow, and switching capacitors. At the same time, while varying power flow parameters, the power flow equations may become inconsistent, and because of this, a physical power flow solution ceases to exist. Such situations correspond to the power flow feasibility limits or power transfer limits. The power flow feasibility limit is determined by points where the power flow Jacobian matrix becomes singular (or equivalently has a zero eigenvalue, or has zero determinant), or where it can be nullified by multiplying by a nonzero vector.

\footnotetext{
${ }^{38}$ Contributed by Yuri Makarov, PNNL.
} 


\subsubsection{Representational issues ${ }^{39}$}

The number and selection of states in the system state vector allow a complete description of the dynamical behavior of the system. If the system has only three states, then the state vector has three components, it can be visualized as a point in three-dimensional space, and the state space is three-dimensional. A similar concept applies when there are more than three states say $n$ states. The state vector is thought of as a point in an $n$-dimensional state space. An example of state space is the space of nodal voltage magnitudes and angles (Van Cutsem et al. 1998). The parameter space can include any parameters that influence voltage stability. One of the examples of parameter space is the space of nodal active and reactive power injections, such as loads and generation. A system operating point can be represented both in state space and parameter space.

Power injection space: A representation of the point of collapse boundary can include coordinates of voltage magnitudes, voltage angles, real-power injections, and reactive-power injections. Buses index these coordinates. The bus power injections completely specify the power system state via the power system static equations. Therefore the various limits on power system operation can be specified in power injection space. The position of the power injections of an operating point, relative to these limits, gives a representation of the power system security that is complete in the sense that all other representations of the power system security can be computed from it. It is also natural to use power injection space to specify transfers, load changes, redispatch, VAr support, generator, and line flow limits. This justifies the basic hyperplane representation of the point of collapse boundary in power injection space.

Descriptive variable space: It is often useful to augment the set of power injections with other parameters. Then the hyperplanes in the augmented space indicate how the power injection hyperplanes change with parameters. This is used to compute the variation or sensitivity of the hyperplanes to input data and control parameters. Augmentation with states, such as voltage angles and magnitudes, gives a space containing both parameters and states, and corresponding hyperplanes. The coordinates used in such a hyperplane representation of the point of collapse boundary have been named descriptive variables. Descriptive variables are usually a set of parameters describing cutset flows or flowgate flows. More generally, descriptive variables can be described as sets in parameter/state space of interest that are incomplete - in the sense that these sets do not completely specify the power system state. For example, interarea flows are descriptive variables. In the simplest of cases, these descriptive variables may be linear combinations of the coordinates that are available at each bus. There are more complicated representations of these coordinates, in which the power injections may be mapped to descriptive variables in a way that is not one-to-one. The descriptive variables typically are much fewer than the parameters, so many different parameter sets are mapped to a smaller set of descriptive variables. For example, if the descriptive variables are selected cutset flows, then many different sets of power injections yield the same cutset flows.

\subsubsection{Modeling Requirements ${ }^{40}$}

The complete understanding of voltage collapse (Van Cutsem et al. 1998) requires a dynamic model to explain why the voltages fall dynamically. However, some computations concerning voltage collapse require only a static model. If a dynamic model is required, the power system is modeled by a set of differential equations with a slowly changing parameter. Computations that only require static models are advantageous because the results do not require load dynamics

\footnotetext{
${ }^{39}$ Contributed by Yuri Makarov, PNNL.

${ }^{40}$ Contributed by Yuri Makarov, PNNL.
} 
and other dynamics to be known. When using static models to obtain practical results, there is a caveat that there must be a way of identifying the stable operating equilibrium of the power system. In principle, this requires a dynamic model, but the stable operating point is often known either by observing the real power system, by experience, or by knowing the stable operating equilibrium at lower loading and tracking this equilibrium by gradually increasing the loading. Computations associated with voltage collapse that require dynamic models include: (a) predictions of the outcome of the dynamic collapse, (b) any problem involving significant step changes in states or parameters, (c) any computations involving eigenvalues or singular values away from the voltage collapse.

\subsubsection{Existing VSA Methods ${ }^{41}$}

Voltage instability and voltage collapse are complicated phenomena that depend on the interactions of multiple system components and power flow parameters. These include generators; excitation control and over excitation protection; voltage regulators; reactive power sources; components of the transmission and distribution system, such as switching capacitors, under load tap changers; static VAR compensators reaching reactive power limits and loads, such as induction motors; thermostats; manual activities that respond to the decaying voltage and attempt to restore the load to its original demand in spite of decaying voltage; and other static and dynamic load characteristics. It is necessary to distinguish large-disturbance voltage stability, vulnerability to cascading events, and small-disturbance voltage stability.

Large-disturbance voltage stability deals with the system's ability to maintain voltages after disturbances such as generation trips, load loss, and system faults. It is analyzed by modeling long-term system dynamics. Large-disturbance voltage stability is analyzed by solving a set of nonlinear differential or algebraic equations (time-domain simulations or numerical solution) (Kundur 1994, Van Cutsem et al. 1998). The system is considered voltage stable if its posttransient voltage magnitudes remain limited by certain pre-established reliability limits (5 to 10 percent, depending on the severity of disturbance).

Cascading voltage collapse can be caused by a sequence of power system changes as, for example, when groups of induction motors stall in succession, or when a series of generator reactive power limits are reached in succession. For cascading events, the NERC and WECC reliability criteria require the grid planners to evaluate their risk and consequences (WECC 2005, WECC 2005). There are just a few techniques developed to assist in understanding or simulating cascading collapses - see (Hardiman et al. 2004, Ajjarapu and Lee 1998), for example. The main approach seems to be working out the sequence of events of each individual cascading outage with assistance from simulations. The more advanced time-domain simulations can reproduce cascading outages (Van Cutsem et al. 1998).

Small-disturbance stability is concerned with the ability of the system to control voltages following small perturbations or gradual change of parameters such as system load. This type of steady-state stability is analyzed by linearizing nonlinear differential equations at a given operating point (Kundur 1994). Because linear differential equations can be solved analytically, there is no need to solve them numerically. There are many methods to check stability of the linearized system without solving it; that is, by analyzing the matrix of its coefficients $J$ (smallsignal stability matrix or system Jacobian matrix ${ }^{42}$. The most commonly used approach is the

\footnotetext{
${ }^{41}$ Contributed by Yuri Makarov, PNNL.

42 The Jacobian matrix of a set of $n$ functions in $n$ variables is an $n \times n$ matrix of partial derivatives, whose entries
} 
so-called eigenanalysis of matrix $J$ (Kundur 1994). The system is asymptotically stable (has positive damping) if all eigenvalues have negative real parts (are located on the left hand side of the complex plane). It is unstable otherwise (has negative damping). Eigenvalues $\lambda$ are solutions of the characteristic equation $\operatorname{det}(J-\lambda I)=0$, where $I$ is the identity matrix. Alternatively, the eigenvalue problem can be rewritten as follows: $(J-\lambda /) R=0$ or $(\mathrm{Jt}-\lambda /) L=0$, where nonzero vectors $R$ and $L$ are the right and left eigenvectors, and t is the symbol of matrix transposition.

$P-V$ plots represent the load vs. the voltage of a selected bus. The load is defined as the bus load or the total load in an area or the system. P-V curves are calculated using the power flow solutions by increasing the loads step by step. The "nose point" of the curve corresponds to the maximum power that can be delivered to the load. The bus voltage at this point is the critical voltage. If the voltage of one particular bus approaches the nose point faster compared to the other buses, it is assumed that this bus limits the system voltage stability margin.

$V$-Q plots represent the bus voltage versus reactive power of the same bus. To obtain the curve, a particular bus is assumed to be a voltage-controlled bus. A series of power flow simulations are performed for various values of the bus voltage and for the corresponding needed reactive injection. The V-Q curves are obtained by plotting the reactive power injection versus the voltage.

$V$-Q sensitivity analysis is conducted by linearizing the power flow problem and assuming that the active power injections are constant. The linearized system is reduced by eliminating voltage angle increments. The resulting expression links voltage increments with the reactive power increments. The diagonal elements of the inverse, reduced Jacobian matrix are sensitivities of the nodal voltages with respect to reactive power injections at the same buses. Large sensitivity indicates reduced stability margin. Negative sensitivity indicates instability.

Q-V modal analysis is based on the analysis of eigenvalues of the reduced Jacobian matrix. The magnitude of the eigenvalues gives the relative measure of the proximity to instability. When the system reaches instability, the modal analysis helps identify the voltage instability areas and the elements that participate in each instability mode (eigenvalue).

Bus participation factors determine the buses associated with each stability mode. The size of the bus participation factor indicates the effectiveness of remedial actions in stabilizing the corresponding mode.

Branch participation factors (calculated for each mode) indicate which branches consume the most reactive power in response to an incremental change in reactive load.

Generator participation factors indicate which generators supply the most reactive power in response to an incremental change in reactive power loading.

There is a variety of methods for voltage stability/security analysis. Universities, research and development organizations, individual developers, and some vendors propose dozens of different promising methods and modifications. At the same time, the industry has accepted just a few of these approaches as standard methods (i.e., the most traditional approaches such as

are the derivatives of each function with respect to each variable.

WESTERN ELECTRICITY COORDINATING COUNCIL - WW W. WECC.BIZ 155 NORTH 400 WEST • SUITE 200 • SALT LAKE CITY • UTAH • 84103-1114 PH 801.582.0353 
$\mathrm{P}-\mathrm{V}$ and $\mathrm{V}-\mathrm{Q}$ simulations, and transient stability time-domain simulations), leaving the rest of the methods to serve as experimental or supplementary tools.

In the next section, these techniques will be outlined using the static voltage stability models, with an emphasis on saddle node bifurcations.

\subsubsection{Stressing algorithms in a specified loading direction ${ }^{43}$}

Step-by-step loading: Traditional power flow calculation methods, such as the Newton-Raphson method, are not capable of determining the voltage stability boundary point directly and accurately. They diverge before the point of collapse is reached. The idea of the step-by-step loading is to exploit the quadratic convergence of the Newton-Raphson method in the vicinity of a solution. The procedure starts from a balanced power flow by incrementing the nodal power injections in a specified stress direction using some initial step size. If the Newton-Raphson method converges, the increment is repeated. In case of divergence, the step is divided by two, and in so doing, the next solution point is brought closer to the solution that is already found along the loading direction. The procedure stops when the step size becomes smaller than some specified accuracy. This method allows the use of detailed power system model, including an accurate modeling of equipment limits (such as generator capability limits, switching capacitor limits, transformer tap changer limits, and others) and discrete controls (such as transformer and switching capacitor steps). Computational divergence is not the best criterion to determine the point of collapse because it can be caused by different reasons (Gurevich et al. 1990, Austria et al. 1995, Gao et al. 1996, Sauer and Lesieutre 1995).

Step-by-step loading with the analysis of a static, small-signal stability criterion: In this method, instead of or in addition to the power flow divergence criterion, the determinant (or an eigenvalue with the maximum real part, or the maximum singular value, (Löf et al. 1992) or the distance between closely located, power flow solutions (Tamura et al. 1983) of the small signal stability matrix $J$ is calculated at each loading step. The moment when the determinant of $J$ changes its sign is considered as the saddle node bifurcation point. This approach also helps determine the small-signal stability boundary points corresponding to the saddle node or Hopf bifurcations - if these points are met before the power flow feasibility boundary is reached.

Permanent or continuous loading: This technique uses the Matveev's method for solving the power flow problem (Matveev 1964). It has been shown that the Matveev's numerical method always converges to a solution or to a point where the power flow Jacobian matrix is singular. The permanent loading (or continuous loading) algorithm (Tarasov 1975) exploits that characteristic of the method. In this approach, the loading parameter is set large enough to make sure that the power flow problem does not have a solution (the point is outside of the power flow feasibility boundary in the parameter space). Beginning from the operating point, Matveev's method starts to iterate producing the sequence of points. Approaching the boundary, the step size becomes continually smaller. When the step size becomes small enough, the process is stopped in the vicinity of the power flow feasibility boundary. Because of linearization, the final point is not exactly the point where the stress vector intersects the power flow feasibility boundary. To eliminate this deviation, a modification of the permanent loading procedure is proposed (Kontorovich et al. 1982, Kontorovich and Makarov 1989). In this modification, the permanent loading steps play a role of a "predictor." If the iterative process deviates too much from the loading direction, a "corrector" step is performed. Alternatively, the

\footnotetext{
${ }^{43}$ Contributed by Yuri Makarov, PNNL.
} 
permanent loading process is continued to the point of singularity, and only then is the "corrector" step implemented. This approach is one of the most commonly recognized and frequently used techniques in the industry.

Parameter continuation predictor-corrector methods are the most reliable power flow methods that are capable of reaching the point of collapse on the power flow feasibility boundary. The addition of new variables, called continuation parameters, determines the position of an operating point along some power system stress direction in the parameter space. The predictor step consists of an incremental movement of the power flow point along the state space trajectory, based on the linearization of the model. The corrector step, which follows each predictor step, lies in the reduction of the linearization error, which is accomplished by balancing the power flow equations to a close point on the nonlinear trajectory.

The main steps of the algorithm are illustrated in Figure 11.

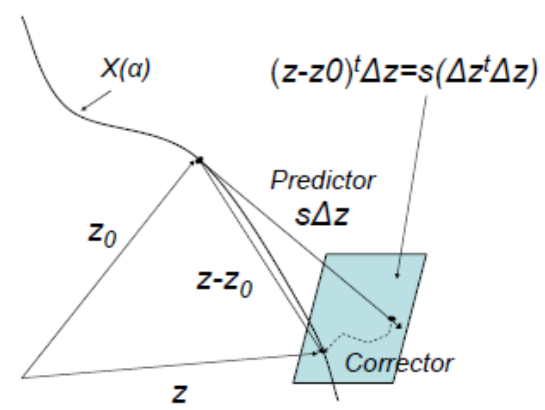

Figure 11. Predictor-corrector process (Parashar et al. 2007a).

Points on the power flow solution space boundary are described by a set of $2 n+1$ equations in $2 n+p$ unknowns.

$$
\begin{gathered}
f(x, \lambda)=0 \\
f_{x}(x, \lambda) y=0 \\
y^{t} y=1
\end{gathered}
$$

Algorithm for Parameter continuation predictor-corrector methods is presented in Appendix 14.

\subsubsection{Stressing algorithms in the most critical direction ${ }^{44}$}

Methods for finding the PoC (point-of-collapse) in the most critical direction employ the same ideas as the direct methods. The difference is that the stress direction in the parameter space is not fixed, and an additional condition requiring that the system stress vector will be a perpendicular vector with respect to the power flow feasibility boundary at the point of collapse is applied. This direction is called the critical direction determining the shortest distance to

\footnotetext{
${ }^{44}$ Contributed by Yuri Makarov, PNNL.
} 
instability. The critical direction coincides with the direction of the left eigenvector, nullifying the power flow Jacobian at the closest point of collapse (Kundur 1994, Kontorovich et al. 1988, Dobson and Lu 1992, Dobson et al. 1991, Dobson and Lu 1993). By applying this approach, one can evaluate the worst-case stress direction and the corresponding critical voltage stability margin for a given operating point in the parameter space. This is useful additional information for VSA purposes. At the same time, there are potential problems with this technique that need to be addressed in practical calculations:

- The critical loading direction might be unrealistic or unlikely.

- Because of the nonlinear shape of the power flow feasibility boundary (besides the critical directions), some subcritical system stresses with a comparable voltage stability margin might be observed (Makarov et al. 1997). In this situation, the critical loading direction does not provide a sufficient characterization of the voltage stability margin.

- The subcritical stress directions correspond to the local minima of the distance-toinstability metric.

This method does not inform whether the result corresponds to the global or local minimum, what the other directions are, and how many of them exist.

Parameter continuation methods for exploring power flow feasibility boundary. The robust predictor-corrector procedure can be applied successfully to explore the entire structure of the power flow feasibility boundary. Points on the solution boundary are described in the same way that it is done in the direct method: using the power flow equations together with an equation that forces the power flow Jacobian to be singular. Contours describing the boundary are obtained by freeing two parameters of the system and following these contours (Hiskens and Davy 2001).

High-order methods to follow the power flow feasibility boundary. The Newton-Raphson method is based on linearization of the power flow equations at each iteration. The high-order method is a generalization of the Newton-Raphson method involving nonlinear terms of the Taylor series expansion (Makarov et al. 1995). It also can be considered as a parameter continuation technique. The method provides a reliable solution to nonlinear algebraic problems, up to points of singularity; there is convergence to a singular point if it occurs on the way of the iterative process; almost a straight line motion of the iterative process in the space of power flow mismatches; and retention of zero mismatches. Once an initial point on the power flow feasibility boundary is found, further exploring of the boundary can be done by changing the stress vector in the direction of interest and by applying the direct method for exploring the boundary. Because the singularity equation $\mathrm{J}^{*} \mathrm{R}$ is equal to zero at the initial point, the high-order method keeps it near zero during the iteration process. This means that the solution point is automatically kept on the power flow feasibility boundary (Makarov and Hiskens 1994). The advantages of the analyzed techniques are that they do not require repeating the loading process, and that they do not require multiple calculations of multiple interior points of the voltage security region to reveal parts of the power flow feasibility boundary.

There are some approximation techniques available for security regions discussed in Appendix 15. 


\subsubsection{Overview of existing tools ${ }^{45}$}

$\mathrm{P}-\mathrm{V}$ and $\mathrm{V}-\mathrm{Q}$ simulation capabilities are provided primarily by industrial-grade VSA tools, including ABB-VSA, PSS/TPLAN (Siemens), VSAT (Powertech), VSTAB (EPRI) and other applications, as described in this section. An overview of voltage security assessment tools is provided below.

- $\quad A B B$ Voltage Security Assessment (ABB-VSA) - This application computes the voltage collapse $\mathrm{P}-\mathrm{V}$ curves and critical operating megawatt limit for increasing loading conditions both for the real-time network condition as well as for the worst contingencies (Masiello 2004). In addition to the prediction of this critical point, ABB-VSA determines the set of weakest load-flow buses in the system that exhibit the worst voltage drops, thus contributing to voltage collapse.

- $\quad P S S / E$ (Siemens) - PSS/E includes an additional, fully automated feature that allows the user to determine real-power transfer or load-level limit using P-V analysis, or determine reactive margin with $\mathrm{V}-\mathrm{Q}$ analysis. For the automatic contingency analysis, the TPLAN non-divergent power flow is used. For the automatic $P-V$ and $V-Q$ analyses, the IPLAN language ${ }^{46}$ script is used. For the post-contingency $P-V$ and $V-Q$ analyses, the inertia/governor load flow algorithm is used. In this algorithm, the speed governor action is modeled, as is all automatic actions controlling voltages and frequency in the 0- to 3minute time frame (Xu et al. 2002).

- VSAT (Powertech Labs, Inc.) - The Powertech voltage security software provides the following capabilities: contingency analysis based on voltage security margin; transfer limits calculation between a source and a sink, and between any three sources/sinks, voltage level, reactive power, and thermal limits; P-V and V-Q analyses; modal analysis; and remedial actions. Powertech also has developed a near, real-time application of the DSA tools (Powertech 2008, PowerWorld undated).

- $\quad \operatorname{VSTAB}(E P R I)$ - VSTAB uses power flow-based, steady-state techniques for stability analysis.

- VSTAB automates contingency analysis and conducts P-V and V-Q simulations. VSTAB also performs a modal analysis by calculating the smallest eigenvalues (EPRI 1993).

- NEPLAN / Voltage Stability (BCP Busarello+Cott+Partner, Inc., Switzerland) - NEPLAN software implements $\mathrm{V}-\mathrm{Q}$ analysis, $\mathrm{P}-\mathrm{V}$ analysis, $\mathrm{V}-\mathrm{Q}$ sensitivity analysis, and modal analysis functionalities. NEPLAN - Voltage Stability helps to identify weak buses, areas, branches, voltage sensitivity, and voltage stability indices. The tool also allows selecting measures to increase the voltage stability margin (BCP Switzerland undated).

- Power World Simulator/PVQV - Power World Corporation's PVQV (PV curve and QV curve) simulator solves the task of evaluating the maximum transfer between userspecified groups of generators and loads. This feature is implemented as a source-sink type of loading. Both voltage-stability and voltage-level limits are accounted for in the procedure. The voltage collapse point is identified as the point where a power flow

\footnotetext{
${ }^{45}$ Contributed by Yuri Makarov, PNNL.

${ }^{46}$ The IPLAN language is used to control the host PSS/E program.
} 
solution cannot be found. The simulator also provides integrated contingency analysis (EPRI 1992).

\subsubsection{Some limitations of existing tools}

Many existing tools use the power flow existence criterion to compute the boundary. The danger with this criterion is the potential of overestimating the actual voltage security margin in situations where the saddle node bifurcation, Hopf bifurcation, or transient stability conditions are violated before the power flow equations become divergent.

The limitations of PV/QV plots, which represent the load versus the voltage of a selected bus, become apparent when voltage collapses are not concentrated in a few buses. Some voltage collapses are regional or involve the entire system. P-V curves are calculated using the power flow solutions by increasing the loads step-by-step. The "nose point" of the curve corresponds to the maximum power that can be delivered to the load. The bus voltage at this point is the critical voltage. If the voltage of one particular bus approaches the nose point faster than the other buses, it is assumed that the system voltage stability margin is limited by this bus. This information does not capture the extent to which all the variables participate in the voltage collapse.

\subsubsection{Impacts of variable generation on voltage stability ${ }^{47}$}

Conventional generators are an important, reactive power source and contribute significantly in maintaining voltage stability. The increasing penetration of wind farms will displace some of the conventional generators, and their capability to supply reactive power may need to be replaced. By increasing the amount of wind generation in the power system, the system reactive demand is being changed with increasing VAr demand/ supply volatility. As such, wind farms are now required to provide both voltage control and reactive power control capabilities, which are mutually exclusive and cannot be achieved at the same time (Tsourakis et al. 2004).

Fixed wind turbines are not able to provide this reactive support so that reactive support needs to be provided as part of a fixed-speed wind farm's design. Constant-speed wind turbines are equipped with simple induction generators. Induction generators produce active power but consume reactive power. For example, following a disturbance, if the electrical torque cannot overcome the mechanical one, the generator accelerates absorbing a high reactive current resulting in voltage drop (Tsourakis et al. 2004). Variable speed wind turbines, controlled through power electronic converters, are able to regulate their own reactive power and can enhance voltage stability (Tsourakis et al. 2004).

Power quality refers to a range of disturbances that affect consumers, from flickering lamps to blackouts. The major categories of power quality disturbance are harmonic distortion, voltage flicker and voltage steps, and steady-state voltage change.

Wind power is often a source of voltage fluctuating and flicker. The dominating type of wind generators involve induction generators, which do not (without voltage and reactive supporting devices as part of their design) contribute to voltage regulation because they are substantial absorbers of reactive power. Voltage control problems can be solved by installing fixed and dynamic reactive compensation, which improves the voltage stability (Meegahapola et al. 2010).

\footnotetext{
${ }^{47}$ Contributed by David Tovar, EPE.
} 
Power electronic-based reactive power compensation, such as SVC (static VAr compensator) devices and FACTS (flexible alternating current transmission system) devices, can control the voltage in a continuous manner, unlike mechanically switched capacitors/reactors (Leclercq et al. 2003).

"The WECC Wind Generation Task Force (WGTF) has proposed to establish for all generators the echnologyneutral standard shown in Figure 12, which will address high and low voltages during the fault and the recovery period. A detailed discussion of the WGTF analysis is available in a WECC white paper titled, "The Technical Basis for the New WECC Voltage Ride-Through Standard," which was prepared by WECC in 2007. The proposed WECC standard is a step forward with its more-comprehensive coverage of the postfault voltage recovery period, the coverage of overvoltage requirements, and the applicability to all (future) generators. This proposed WECC standard may be more appropriately adopted as an NERC continental standard" (Zavadil, R. et. al. 2007).

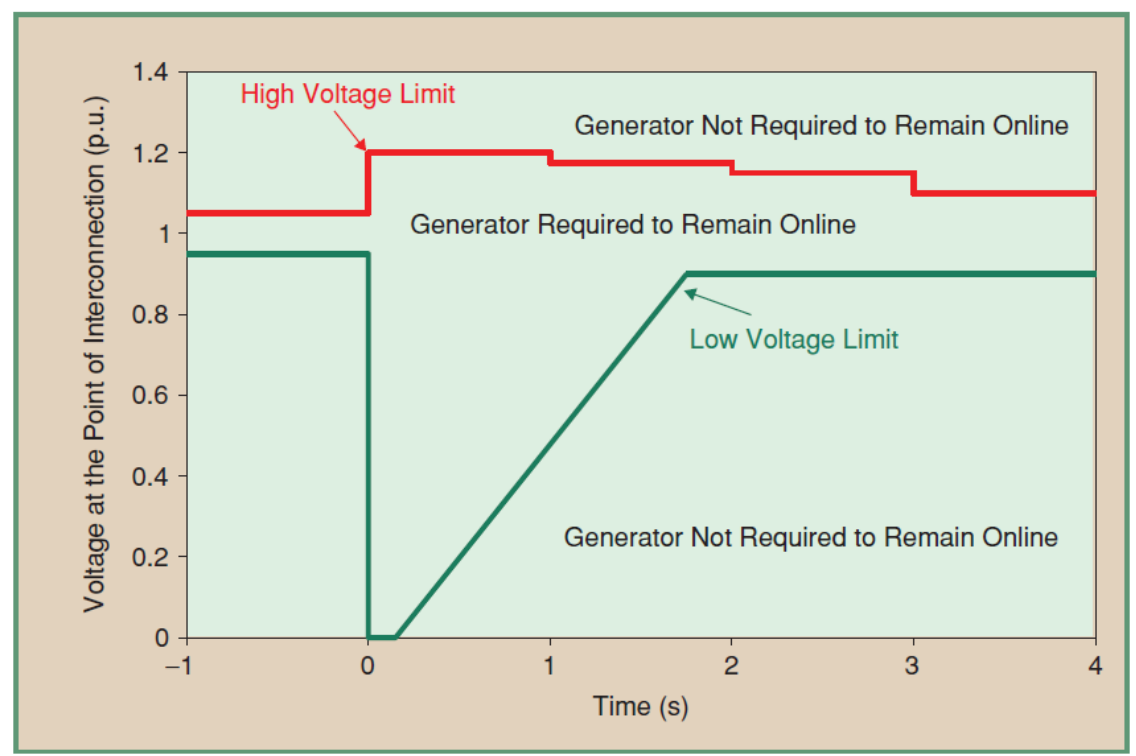

Figure 12. Proposed WECC voltage ride-through requirements for all generators (Zavadil, R. et. al. 2007) ${ }^{48}$.

"Older wind generators based on simple induction machines create a reactive power burden for the power system. They often degrade system voltage performance rather than support it. FERC Orders 661 and 661A address the need for wind plants to support power system voltage by requiring new wind generators to have the capability to control their reactive power within the 0.95 leading to 0.95 lagging range. Recognizing that providing this capability can be expensive, FERC only requires it if the interconnection study shows that it is needed. The interconnection study also determines if power electronics are needed for dynamic control or if switched capacitors are sufficient. Many modern wind generators provide this dynamic capability directly from the power electronics that control the real power operation of the machine. These plants can provide excellent voltage control for the power system" (Zavadil, R. et. al. 2007).

\footnotetext{
${ }^{48}$ Reused by permission of IEEE.
} 


\subsubsection{Small signal stability ${ }^{49}$}

Small-signal stability (SSS) is concerned with the ability of the system to control its parameters following small perturbations, or gradual change of parameters, such as system load. Smallsignal stability is a subset of rotor-angle stability, which deals with the ability of synchronous machines interconnected through an electrical power system to remain in synchronism after a small disturbance or small disturbances on that system. Small-signal instability problems after a small disturbance, or small disturbances on an electrical power system, can occur because of a steady increase in rotor angles.

\subsubsection{Modes of electromechanical oscillations ${ }^{50}$}

The types of electrical power system electro-mechanic oscillations can be divided into four groups:

1. Local modes $(0.7$ to $3 \mathrm{~Hz})$ - oscillations between generators in the power plant (1.5 to 3 $\mathrm{Hz}$ ), or oscillations between an aggregate power plant and neighboring parts of the electrical power system $(0.7$ to $1.5 \mathrm{~Hz})$;

2. Interarea modes $(0.1$ to $0.7 \mathrm{~Hz})$ - oscillations of a group of synchronous machines in one part of the system against another group of machines in another part of the system;

3. Control modes $(>4 \mathrm{~Hz})$ - oscillations caused by the control of synchronous machines (governors and exciters) or control of equipment such as high-voltage, direct current (HVDC) and static VAr compensator (SVC) devices; and

4. Torsional modes $(>4 \mathrm{~Hz})$ - oscillations caused by the interaction between a turbinegenerator shaft system and another system element (e.g., a series capacitor compensated line) (Bragason 2005, Bebic 2008).

\subsubsection{Impact of wind generators ${ }^{51}$}

The impact of wind turbine generators (WTGs) on SSS is that WTGs that are not coupled directly to the electrical power system (variable speed WTGs) do not contribute to low frequency directly, but they may have an influence on these indirectly. Fixed-speed WTGs can regulate power via pitch control, and therefore can contribute to system damping (Sun et al. 2010).

The impact of WTGs of small-signal stability depends on their type. As described in Section 2.1.1, there are several major kinds of WTGs in the market nowadays, namely squirrel cage induction generators (SCIGs), DFIGs, and direct-driven synchronous generators (DDSGs). SCIGs are known as fixed or constant-speed WTGs (CSWTGs), contrasted by DFIGs and DDSGs, which are treated as variable speed WTGs (VSWTGs). The major discrepancies of the WTGs are the electrical subsystem and the way that the aerodynamic efficiency of the wind turbine rotor of the mechanical subsystem is limited at high wind speeds (Slootweg 2003). The key features of these WTGs are summarized below (Slootweg 2003, Li and Chen 2008):

\footnotetext{
${ }^{49}$ Contributed by David Tovar, EPE, Jian Ma, PNNL, and Yuri Makarov, PNNL.

50 Contributed by David Tovar, EPE.

${ }^{51}$ Contributed by David Tovar, EPE, and Jian Ma, PNNL.
} 
- Squirrel cage induction generator (SCIG) - The wind turbine rotor is connected to the generator through a gearbox, and the stator is directly coupled to the grid with a shunt capacitor bank. At high wind speeds, the mechanical power extracted from the wind can be restricted by stall control or active stall control. Although SCIGs are simple, robust and low cost, they have some drawbacks: (i) the lack of capability to control active and reactive power independently, (ii) higher mechanical stress, (iii) they require gearboxes maintenance, and (iv) low-efficiency energy conversion.

- DFIGs - The wind turbine rotor is connected to the generator through a gearbox. The stator is directly coupled to the grid, but the rotor connects the grid through a partialscale converter. At high wind speeds, the mechanical power extracted from the wind can be restricted by pitch control. In comparison to SCIGs, DFIGs have the following advantages: (i) the flexibility to control active and reactive power, (ii) maximum power point tracking (MPPT) ability by speed controller, (iii) reduced mechanical stress, and (iv) a smaller converter scale. But they still require the maintenance of gearboxes and are more expensive than SCIGs.

- $\quad$ DDSGs - The wind turbine rotor is connected directly to the generator, and the stator is coupled to the grid through a full-scale converter. At high wind speeds, the mechanical power extracted from the wind can be restricted by pitch control. They are similar to DFIGs in certain ways: i) the flexibility to control active and reactive power, ii) MPPT ability via speed adjustment, and iii) reduced mechanical stress. In addition, DDSGs can operate without gearboxes, but they are heavier, and the converters are larger than DFIGs.

In recent years, several research efforts have been devoted to address the impact of wind power on power system small-signals stability, and these details are discussed in Appendix 16.

\subsubsection{Fault-induced delayed voltage recovery ${ }^{52}$}

The NERC Transmission Issues Subcommittee (TIS) has examined, described, and defined fault-induced, delayed voltage recovery (FIDVR) as follows: (NERC 2009b)

"A delayed voltage recovery event - more commonly known as a fault-induced, delayed voltage recovery (FIDVR) event - is the phenomenon whereby system voltage remains at significantly reduced levels for several seconds after a transmission, sub-transmission, or distribution fault has been cleared. Significant load loss because of motor protective device action can result, with the potential for a secondary effect of high system voltage because of load loss. A severe event can result in fast voltage collapse.

FIDVR is a voltage condition initiated by a fault and is characterized by:

- Stalling of induction motors;

- Initial voltage recovery after the clearing of a fault to less than 90 percent of precontingency voltage; and

\footnotetext{
${ }^{52}$ Contributed by Yuri Makarov, PNNL.
} 
- Slow voltage recovery of more than 2 seconds to expected post-contingency steadystate voltage levels.

Additional information on FIDVR is presented in Appendix 17.

\subsubsection{Possible grid planning solutions (NERC 2009b)}

The solution to FIDVR would be to install equipment control devices to remove AC and other induction motor loads from the grid prior to stalling for undervoltage conditions. This must be a component of a long-range solution. This equipment protection could be a first step toward the application of additional reactive sources, such as SVCs, to specifically protect against distribution system voltage variations. However, AC standards necessary to achieve this will not be enacted overnight, and retrofit of existing $A C$ units would likely be even more difficult to accomplish. It could also be argued that system exposure to FIDVR may be a symptom of a larger issue: inadequate dynamic reactive support. Consequently, the use of grid solutions will be necessary at least until the threat level of $A C$ loads can be reduced. These grid solutions cannot ensure prevention of FIDVR events, but they may contain events by limiting both their area of influence and their consequences. In Appendix 18, some of the grid level solutions are discussed.

\subsection{Grid Controllability ${ }^{53}$}

This section considers various options available for controlling grid power flows, maintaining secure ranges of system parameters, ensuring its reliability, and helping to improve the utilization of existing transmission facilities.

\subsubsection{FACTS $^{54}$}

FACTS is an acronym that stands for flexible AC transmission system. The concept of this technology was introduced by Hingorani in 1988 (Hingorani 1988). FACTS Terms and Definitions Task Force (1997) provides the following definition: "Alternating current transmission systems incorporating power electronic-based and other static controllers to enhance controllability and increase power transfer capability."

"From an economical point of view, more power can be transmitted over existing or new transmission grids with unimpeded availability at an investment cost and time expenditure lower, or in cases even much lower than it would cost to achieve the same with more extensive grids. Also, in many cases, money can be saved on a decrease of power transmission losses.

From an environmental point of view, FACTS enables the transmission of power over vast distances with less or much less right-of-way impact than would otherwise be possible. Furthermore, the saving in transmission losses may well bring a corresponding decrease in need for generation, with so much less toll on the environment" (International Council on Large Electric Systems undated).

Flexible AC transmission system devices can ensure transmission system stability and increase the available capacity at key points in the transmission network by routing power flows. Unified

\footnotetext{
${ }^{53}$ Contributed by David Tovar, EPE, Pavel Etingov, PNNL, and Yuri Makarov, PNNL.

${ }^{54}$ Contributed by Pavel Etingov, PNNL.
} 
power flow controllers (UPFCs) and phase-shifting transformers are able to directly route active power flows through the transmission network, and route power away from lines that require additional capacity. Furthermore, UPFCs are able to provide voltage support through functionality similar to SVCs and Static synchronous compensators (STATCOM). Series compensation can be used to increase the available capacity on a given transmission path.

When there is more than one transmission path between a generator and a load, the power flows preferentially through the transmission grid down the path of least impedance. Series compensation can reduce the static and dynamic impedance of a transmission line, thus increasing the load carried by that line, and reducing the load carried by parallel paths. Static VAr compensators (SVCS) and STATCOM can provide dynamic reactive power to serve as voltage support to counteract changes to system voltage. These devices can improve system stability, and they do not increase the available transmission capacity directly. However, these devices can indirectly increase transmission capacity because, with the improved control over system stability, transmission lines can be operated closer to their limits (Holman 2011).

The FACTS devices can provide the following benefits to the system:

- Improved power transmission capability.;

- Better voltage and reactive power control;

- Improved system stability and availability;

- Damping of oscillations;

- Improved power quality;

- Minimized environmental impact; and

- Minimized transmission losses (International Council on Large Electric Systems undated, Alberta Energy 2009).

\subsubsection{FACTS controllers' categories}

According to Hingorani and Gyugyi (2000), there are four FACTS controllers' categories:

\section{Shunt controllers such as SVC and STATCOM}

"These systems operate by sensing the AC line voltage and linearly increase their inductance (and at the same time reduce their capacitance) to reduce the AC line voltage or linearly decrease their inductance (and at the same time increase their capacitance) to increase the AC line voltage. These increases/decreases can be achieved very dynamically in order to quickly react to changes in the AC line to maintain stability" (Alberta Energy 2009).

"As in case of series controllers, the shunt controllers may be variable impedance, variable source, or a combination of these. In principle, all shunt controllers, as shown in Figure 13, inject current into the system at the point of connection" (Hingorani and Gyugyi 2000). 


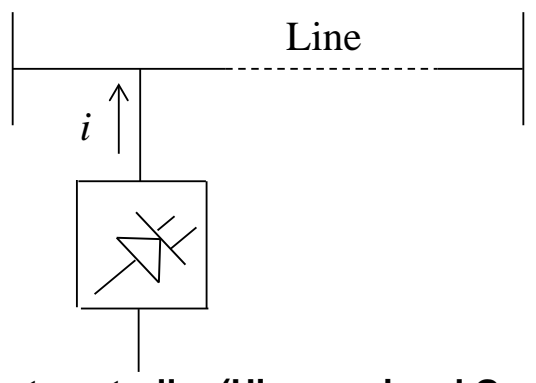

2. Series controllers such as TCSC

Figure 13. Shunt controller (Hingorani and Gyugyi 2000).. ${ }^{55}$

"Series connected systems involve connecting capacitance in series with the transmission line. This series capacitor effectively cancels part of the line inductance to reduce the transfer impedance of the line, which in turn significantly increases the power transfer capacity of the line. Additionally, the series capacitor system can:

- Control the sharing of power between parallel, connected lines;

- Provide reactive power to the circuit;

- Maintain the phase angle across transmission line within safe limits to ensure angular stability; and

- Enhanced voltage stability along the line" (Alberta Energy 2009).

"The series connected systems have to be built on platforms insulated at the voltage level of the transmission line itself. If power electronics are used in association with the series capacitance (see TCSC), then the system can act fast enough to provide damping of power oscillations to improve voltage stability and prevent sub-synchronous resonance, which could cause damage to the shafts of any locally connected generators" (Alberta Energy 2009).

"The simplest form of series compensation is known as fixed series compensation (FSC), the main benefit of which is to allow an increase in transmission capacity. The more sophisticated TCSC (thyristor-controlled series compensation) is deployed if fast control of the line impedance is required for load-flow control and for damping power oscillations. It also has the benefit of enabling the mitigation of sub-synchronous resonances" (Alberta Energy 2009).

Figure 14 shows a series controller. "It could be variable impedance, such as capacitor, reactor, etc., or power electronics based variable source of main frequency, subsynchronous and harmonic frequencies (or a combination) to serve the desired need. In principle, all series controllers inject voltage in series with the line" (Hingorani and Gyugyi 2000).

\footnotetext{
${ }^{55}$ Reused by permission of IEEE.
} 


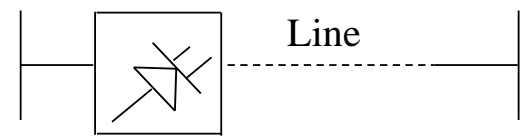

Figure 14. Series controller (Hingorani and Gyugyi 2000). ${ }^{56}$

\section{Combined series-series controllers}

"This could be a combination of separate series controllers, which are controlled in a coordinated manner. Or it could be a unified controller in which series controllers provide independent series reactive compensation for each line but also transfer real power among the lines via the power link as shown in Figure 15" (Hingorani and Gyugyi 2000).

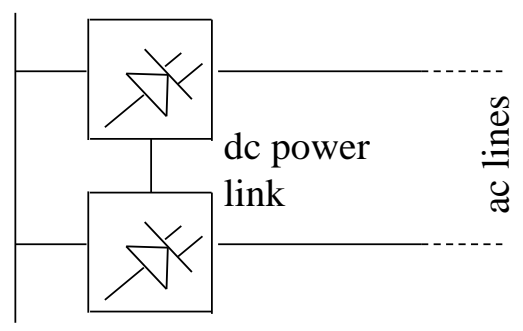

Figure 15. Combined series-series controller (Hingorani and Gyugyi 2000).. ${ }^{57}$

\section{Combined series-shunt controllers}

"This can be a combination of separate shunt and series controllers that are controlled in a coordinated manner, or a unified power flow controller with series and shunt elements as shown in Figure 16" (Hingorani and Gyugyi 2000).

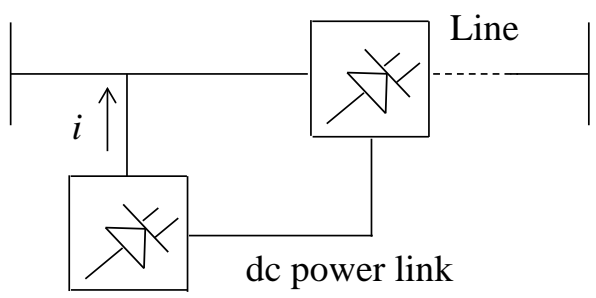

Figure 16. Combined series-shunt controller (Hingorani and Gyugyi 2000).. ${ }^{58}$

The full list and definitions of FACTS controllers can be found in FACTS Terms and Definitions Task Force (1997).

"Important applications in power transmission and distribution involve devices such as static VAr compensators (SVC), fixed series capacitors (SC) as well as thyristorcontrolled series capacitors (TCSC) and static synchronous compensator (STATCOM).

\footnotetext{
${ }^{56}$ Reused by permission of IEEE.

${ }^{57}$ Reused by permission of IEEE.

${ }^{58}$ Reused by permission of IEEE.
} 
In Appendix 19, descriptions of several commonly used FACTS devices are provided.

\subsubsection{Applications of specific FACTS devices}

The use of FACTS for various applications is summarized in Table 3 (International Council on Large Electric Systems undated).

Table 3. FACTS applications.

\begin{tabular}{|l|l|}
\hline \multicolumn{1}{|c|}{ Problem } & \multicolumn{1}{|c|}{ Device } \\
\hline Steady-state voltage control & SVC \\
\hline Dynamic voltage control & $\begin{array}{l}\text { SVC } \\
\text { STATCOM }\end{array}$ \\
\hline Transient stability & SVC \\
& TCSC \\
\hline Power oscillation damping & SVC \\
& TCSC \\
\hline Power quality & SVC \\
& STATCOM \\
\hline $\begin{array}{l}\text { Sub-synchronous } \\
\text { resonance }\end{array}$ & TCSC \\
\hline
\end{tabular}

The prices for different FACTS technologies are listed in Appendix 19.1.

\subsubsection{FACTS application for wind generation}

"The dominating kind of wind power generation is asynchronous, this since it is robust and cost effective. Induction generators, however, do not contribute to regulation of grid voltage, and they are substantial absorbers of reactive power. Ideally, they need to be connected to stiff grids in order not to influence power quality in a detrimental way. This is usually not the case, however. Quite on the contrary, wind power is usually connected far out in the grid, on sub-transmission or distribution levels, where the grid was not originally designed to transfer power from the system extremities back into the grid.

The reactive power balance of asynchronous generators can be improved to a certain extent by use of the doubly fed rotor concept. To keep this technology within reasonable cost margins, however, rotor converter ratings must be kept limited to steady-state requirements only. During transient occurrences in the grid, the performance of DFIGs may well prove inadequate to safeguard primarily voltage stability of the grid, possibly even with a necessity to crowbar the rotor converters to protect them from overload. In a situation like that, the DFIG will basically be reduced to a common induction generator. Here, dynamic, reactive power support from FACTS in the point of common coupling (PCC) can improve the situation greatly.

For off-shore wind generation, comprehensive AC sea cable networks add another dimension, calling for additional elaborate reactive power control. The overall scope of reactive power control should encompass the wind farm just as well as the sea cables, to bring about a wellregulated, reactive power balance of the whole system, answering to the same demands on reactive power regulation as any other medium to large generator serving the grid" (Grünbaum 2010). 
In many countries (such as Germany, United Kingdom, and Denmark), there is a set of special requirements (grid codes) applicable to wind generation. A review of grid code requirements in different countries is provided in Singh and Singh (2009). In general, these requirements include:

- Reactive power supply;

- Voltage control;

- Fault ride-through capability;

- Power quality control (flicker, harmonics); and

- Frequency control (Grünbaum 2010, Singh and Singh 2009, Adamczyk et al. 2010).

FACTS technology can help wind power plants meet these requirements.

\section{Reactive power supply and voltage control}

"Grid code requirement for steady-state and transient reactive power support originates in voltage stability problems. Reactive power consumption of the connecting lines and loads may lead to a voltage collapse in a weak, heavily loaded system. Such situations are quite typical for wind generation, which is often placed in remote areas and connected with long lines. If reactive power compensation provided by wind power plant is not sufficient, generated active power might need to be limited to avoid voltage instability. It is especially likely for a wind farm employing DFIGs, which not only does not provide compensation but also consumes reactive power" (Adamczyk et al. 2010). SVC and STATCOM can be used to continuously and smoothly control reactive power and voltage at the PCC.

"In cases of off-shore wind farms, where $A C$ is utilized to bring the power to shore, the sea cable(s) must be included in the overall picture, as well as the reactive power generated in the cable(s), which will influence the power factor at the PCC. With FACTS, this can be accommodated in a natural way, for all operating situations of the wind farm, including the case where the wind farm is idle or even disconnected (Figure 17). In fact, AC is the predominant way of landing power from off-shore wind farms. In Denmark alone, two off-shore wind farms, each rated at more than $150 \mathrm{MW}$, are connected to shore by means of AC. With modern cables, the break-even distance between AC and DC lies beyond 100 km" (Grünbaum 2010). 


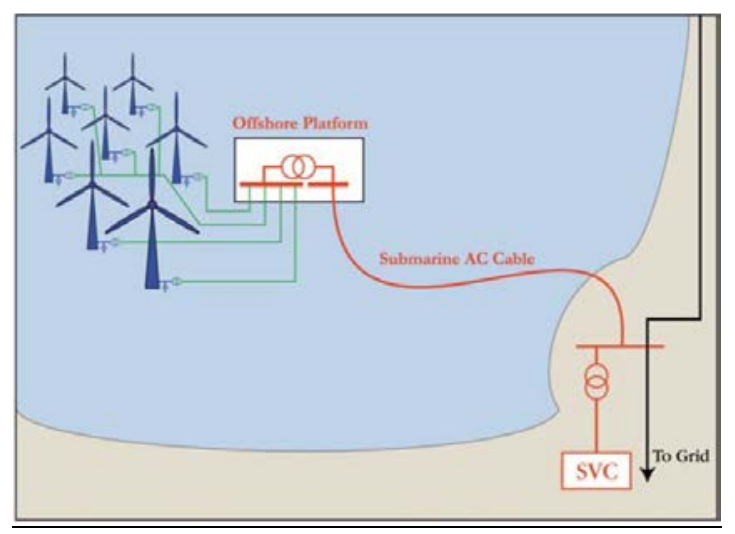

Figure 17. SVC and off-shore wind power plant (Grünbaum 2010) ${ }^{59}$.

\section{Fault ride-through capability}

"Regarding fault ride-through, the e.on (Germany) grid code stipulates that the wind turbine generator must stay connected for a close-up, three-phase fault in the transmission system that is cleared within normal protection operating times (150 ms). Mechanical power output during and after the fault has been cleared must not be significantly reduced (Figure 18).

With SVC, the need for fast injection of reactive power upon fault clearing is readily satisfied. With STATCOM, even faster response is attained" (Grünbaum 2010).

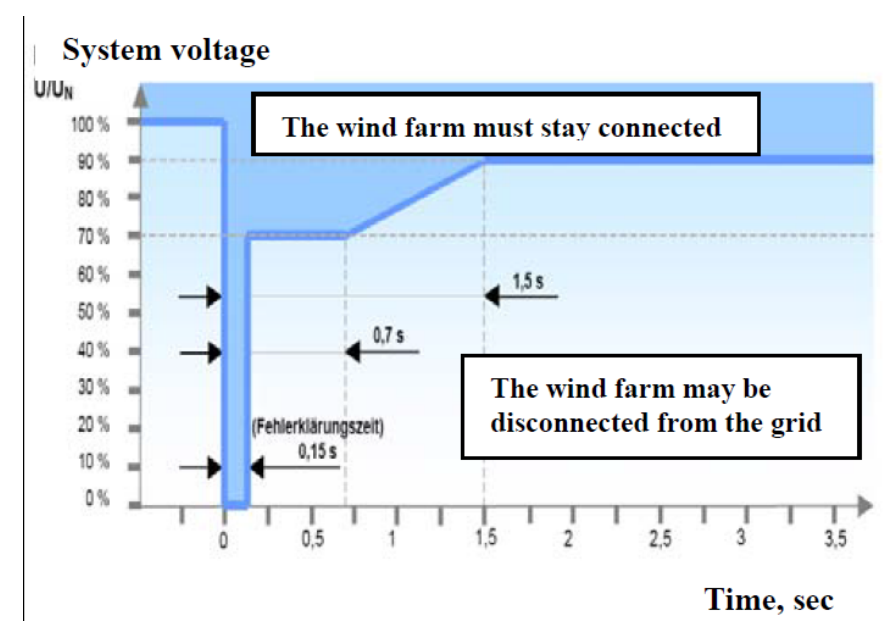

Figure 18. Example of Grid Code requirements: fault ride-through capability (Grùnbaum 2010). ${ }^{60}$

\section{Power quality}

"Another research area is wind power quality improvement with FACTS devices. It is especially attractive in case of DFIG units connected to a weak grid, where changing wind speed causes voltage fluctuations at a wind farm's point of common coupling (PCC) and flicker. In Han et al. (2008) it is shown that dynamic reactive power compensation device like STATCOM can solve this problem. Very interesting issues are studied in Bagnall et al. (2009). Capacitances of lowloss cables that are used in wind farms together with main transformers inductance form poorly

\footnotetext{
${ }^{59}$ Reused by permission of IEEE.

${ }^{60}$ Reused by permission of IEEE.
} 
damped resonant tank, with resonance frequency between $11^{\text {th }}$ and 35 th harmonic. By proper controller gain selection, it can be ensured that real part of STATCOM complex impedance is negative for all signals in desired frequency spectrum. That means that STATCOM would absorb active power carried by harmonics and re-inject active power at fundamental frequency" (Adamczyk et al. 2010).

\section{Frequency control}

FACTS devices with energy storage can assist wind power plant to provide frequency response and active power control. For example, coordination of wind farm and STATCOM with a battery energy storage system was used in Baran et al. (2008) for system balancing purpose.

\subsubsection{Phase-shifting transformers}

The location and variability of wind generation (along with the difficulty of financing and gaining permits for the construction of new overhead lines), are pressuring transmission system operators to increase the use of existing transmission capacity as flexibly as possible. Phase shifting transformers are devices that can effectively exercise control on the transmission lines in which they are installed, and can change the distribution of power between different branches of a network.

\subsection{Long-term transmission planning}

Industry transmission planning practices diverge when it comes to "long-term" planning (20+ years). Short-term studies may work well in some applications, but may not adequately identify the long-term needs of the transmission system, and may lead to sub-optimization of the bulk electric system. Many utilities consider "long-term" planning as looking out 10 years. These short study periods and their resulting sub-optimization, given the 75 to 100 year in-service life, may limit the possibility of constructing higher-efficiency, multiple-line systems, and identifying underlying AC system upgrades, to fully realize the reliability and economic benefits a robust transmission system provides (U.S. Department of Energy 2010).

Longer-term plans are developed in less detail than short-term plans. They are concerned with identifying the paths where more transmission capacity is and will be needed rather than how such capacity additions should be realized.

Short-term planning should identify ways of increasing the transmission capacity of the existing grid by modern devices, which are less costly and quicker to install than new lines, and less apt to encounter public resistance. It should evaluate which devices in which locations can best increase transmission capacity. Candidate devices include power system stabilizers, wide-area measurements with phasor measurement units, series compensation (for long lines), and a flexible alternating current transmission system (FACTS, including static VAr compensation, series capacitors, switchable compensation and phase shifting transformers) (EASAC 2009).

Long-term planning requires that transmission system operators (TSOs ${ }^{61}$ ) create a reasonable set of common scenarios for the future. These need to:

- cover demand, generation capacity, and the duration of power flows in lines;

- reflect national plans and incentives;

${ }^{61}$ NERC uses TOP instead of TSO, but not all TSOs would be TOPs. 
- use a common technical basis;

- extend sufficiently far into the future, and do so in such a way that there are significant differences between them; and

- take into account the effects of the characteristics and costs of different methods of generation.

The goal of long-term planning is to develop an optimal investment plan after identifying the remaining constraints for the electricity market. The plan presents a global optimum based on common scenarios and planning principles instead of local optima. The predicted balance between generation and demand in each of the scenarios is the key. Usually it is the high-load situation that is critical, and enough reserve generation and/or transmission capacity are needed for peak-load situations. If the peak load is not simultaneous in different countries, having enough transmission capacity helps stabilize prices. The occurrence and duration of peak-load hours should be estimated for each scenario. The long-term plan enables the identification of critical areas of congestion and, consequently, the best locations for new lines. 


\section{Generation Planning}

This section addresses tail events, variable generation capacity values and important issues concerning overgeneration.

\subsection{Tail events}

Extreme events in the electrical power system, caused by the load and wind forecasting errors, can impact the power system infrastructure via two main avenues. The first avenue is a sudden and significant power imbalance exceeding reasonable operating reserve capacity. The second is a sudden increase of power flows on the system critical paths causing transmission violations. The magnitude of power imbalances and incremental power flows can rise to the level of several thousand megawatts, in cases of extreme wind and load forecast errors occurring concomitantly (Lu et al. 2009). The challenge in managing these system imbalances is more significant for a stand-alone, balancing area operation. The actual or virtual consolidation of Balancing Authorities into a single balancing area can offset the operating reserve problem, but this strategy enhances incremental power flows on the transmission interfaces, potentially leading to more unpredictable transmission congestion (Heydt et al. 2011). Extreme events have been practically observed recently in the United States (Ela and Kirby 2008) and Germany (Ernst et al. 2010).

\subsection{VG capacity value}

Resource adequacy is an important issue with which power system planners contend (Keane et al. 2011). Although there are a variety of technologies to choose from, there is increasing interest in renewables. Some renewables pose capacity planning challenges, however, because of variable and uncertain real-time output (Keane et al. 2011, Milligan 1996, Milligan and Parsons 1999, Milligan and Factor 2000, Milligan and Porter 2006, Pudaruth and Li 2008). Thus, accurate capacity value (CV) estimates of these resources are vital for planning purposes.

\subsubsection{Capacity value analysis methodology}

A capacity value analysis was conducted recently as part of the Western Wind and Solar Integration Study. Using a variety of different metrics, it determined that the capacity value of wind ranges from 10 to 15 percent; from 25 to 30 percent for photovoltaic (PV); and from 90 to 95 percent for concentrated solar power CSP with storage.

\subsubsection{The use of capacity value in planning}

"The appropriate method for computing capacity value needs to be considered in light of the specific context in which the values are to be used. There are state or sub-regionally specific uses of capacity values or approaches to their calculation that may affect the choice of a method.

For instance, there is a specific, regulatory construct for resource adequacy in California. There, resource adequacy makes use of a capacity value known as net qualifying capacity. Each generator is rated according to a specific formula adopted by the decision of the California Public Utilities Commission (CPUC) and the FERC-approved tariff of the California ISO. A unique aspect of this resource adequacy construct is that generators are rated on a month-bymonth basis for each month of the year. Load-serving entities (LSEs) are allowed to satisfy their 
overall resource adequacy requirements (expected monthly peak load plus 15 percent planning margin) by mixing and matching resources separately for each of the 12 months. California to LSES are allowed to select a resource for as little as a month at a time. They can have a separate set of resources from one month to the next. Due to this regulatory construct, LSEs subject to CPUC/CAISO resource adequacy requirements need capacity values for each month for each resource." 62

"The current CPUC approach is based on a detailed set of requirements for calculating net qualifying capacity for variable generation resources. Although ELCC approaches have a theoretical endorsement, no entity has yet proposed how the ELCC method might be meshed into California's resource adequacy construct. An obvious approach - applying the ELCC method on a month-by-month basis - has not yet been evaluated quantitatively to see whether it raises any particular issues for the calculations in California.

The CPUC has a proceeding for review of the adequacy of the existing planning reserve requirement (15 - 17 percent). This proceeding was inactive for roughly 18 months prior to a February 2010 request for comments regarding reopening it. In May 2010, the CAISO submitted a study of the planning reserve margin in the proceeding." 72

\subsubsection{Wind generation capacity value (task force on the capacity value of wind power, 2011)}

"The increase in the penetration of wind generation in recent years has led to a number of challenges for the planning and operation of power systems. A key metric for generation system adequacy is the capacity value of generation" (Task Force on the Capacity Value of Wind Power 2011).

Capacity value is the contribution that a given generator makes to overall system adequacy. "It can be defined as the amount of additional load that can be served due to the addition of the generator, while maintaining the existing levels of reliability" (Task Force on the Capacity Value of Wind Power 2011).

"The metrics that are used for adequacy evaluation include the loss of load expectation (LOLE) and the loss of load probability (LOLP). LOLP is the probability that the load will exceed the available generation at a given time. This criterion only gives an indication of generation capacity shortfall, and lacks information on the importance and duration of the outage. LOLE is the expected number of hours or days, during which the load will not be met over a defined time period. The effective load carrying capability (ELCC) is the metric used in this report to denote the capacity value" (Garver 1966).

\section{Methodology}

"This method is based directly on the definition of capacity value given above. Conventional thermal generation is still the most common form of generation in power systems. They are modeled by their respective capacities and forced outage rates (FOR). Each generator capacity and FOR is convolved via an iterative method to produce the analytical reliability model (capacity outage probability table - COPT) of the power system. The COPT is a table of capacity levels and their associated probabilities (Billinton and Allan 1996). The cumulative probabilities give the LOLP for each possible available generation state. Wind power cannot be adequately modeled by its capacity and FOR, as wind availability is more a matter of resource

${ }^{62}$ WECC, 2011, Variable Generation Capacity Value Draft 
availability than mechanical availability. This leads to a different treatment of wind generation in the traditional ELCC calculation method, which is now summarized in the following three steps:

1. The COPT of the power system is used in conjunction with the hourly load-time series to compute the hourly LOLPs without the presence of the wind plant. The annual LOLE is then calculated. The LOLE should meet the predetermined reliability target for that period. If it does not match, the loads can be adjusted, if desired, so that the target reliability level is achieved.

2. The time series for the wind plant power output is treated as negative load and is combined with the load time series, resulting in a load time series net of wind power. In the same manner as Step 1, the LOLE is calculated. It will now be lower (and therefore better) than the target LOLE in the first step.

3. The load data is then increased by a constant $\Delta \mathrm{L}$ across all hours using an iterative process, and the LOLE recalculated at each step until the target LOLE is reached. The increase in peak load (sum of $\Delta \mathrm{Ls}$ ) that achieves the reliability target is the ELCC or capacity value of the wind plant."

\subsubsection{Solar Generation Capacity Value}

Because of excellent solar-resource availability, the southwestern U.S. has great potential for concentrating solar power (CSP) development, with a number of plants currently operational and others in development. Although the CV of CSP plants without thermal energy storage (TES) has been analyzed (Madaeni et al 2012), TES is a promising technology that can increase the value of CSP by providing a number of services, including increased CVs. Madaeni et al. (2012) proposed a model to optimize the operation of a CSP plant with TES, and applied approximation techniques to estimate the CV of CSP plants at different locations in the southwestern U.S. The study shows that TES can significantly increase the plant's CV — plants without TES have CVs between 61 and 87 percent of maximum capacity, whereas adding 1 hour of TES can increase the CV to between 79 and 90 percent. The study also examines the effect of capacity payments, demonstrating that they can increase the CV of CSP, because energy prices are not a perfect signal of capacity scarcity.

\subsection{Generation fleet flexibility ${ }^{63}$}

In April 2009, the NERC Integration of Variable Generation Task Force (IVGTF) released its report: "Accommodating High Levels of Variable Generation" (NERC 2009a). The IVGTF report states, "Resource adequacy and transmission planning approaches must consider needed system flexibility to accommodate the characteristics of variable resources, as part of bulk power system design."

The IVGTF has identified the following system flexibility/reliability functions and services (NERC 2010a):

- Inertial Response: Very fast response (cycles of 1 to 2 seconds), which supports system stability by constraining the initial rate of frequency change following a disturbance.

\footnotetext{
${ }^{63}$ Contributed by Yuri Makarov, PNNL.
} 
- Primary Frequency Response: Very fast response (cycles of 5 to 10 seconds), traditionally from a synchronous generator governor control that adjusts megawatt output as a function of frequency, to arrest frequency deviations following a disturbance.

- Regulation: Continuous response (10 seconds to several minutes) of reserves under automatic generation control (AGC), deployed to correct minute-to-minute deviations in frequency, or return frequency, to the desired range following a disturbance.

- Load Following/Ramping: Slower response (several minutes to few hours) whereby available resources are dispatched to follow system ramping requirements.

- Dispatchable Energy: Dispatchable energy is closely related to load following and ramping, but it focuses on the energy consumption at times of peak capacity requirements, and the minimum load rather than the rate of change in generation and consumption.

- Contingency Spinning Reserve: Generation (or load) that responds immediately to a generator or transmission line failure, and is fully deployed within 10 minutes.

- Contingency Non-spinning Reserve: Similar to spinning reserve except that the response does not need to be immediate, but still fully deployable within 10 minutes.

- Replacement or Supplemental Reserve: Additional reserve that begins responding in 30 to 60 minutes, which is required in some regions.

- Variable Generation Tail Event Reserve: Reserves very similar to conventional contingency reserves that are maintained to cover the large, infrequent ramps of variable generation, and which typically are slower than conventional contingencies.

- Voltage Support: Resources that can provide voltage control to maintain system voltage levels within specified criteria.

\subsection{Operating reserves ${ }^{64}$}

Although the operating reserves are primarily an operational issue, they also should be considered in the planning process. This is an important part of generation fleet optimization, generation adequacy analyses, production cost simulations, and generation fleet flexibility studies.

"The operating reserve is the generating capacity available to the system operator within a short interval of time, in order to meet demand in case a generator goes down or due to another disruption to the supply. Most power systems are designed so that, under normal conditions, the operating reserve is always at least the capacity of the largest generator, plus a fraction of the peak load" (Wang et al. 2005).

The operating reserve is made up of the spinning reserve as well as the non-spinning or supplemental reserve (CAISO 2006).

\footnotetext{
${ }^{64}$ Contributed by Pengwei Du and Yuri Makarov, PNNL.
} 
- The spinning reserve is the extra generating capacity that is available by increasing the power output of generators that are already connected to the power system. For most generators, this increase in power output is achieved by increasing the torque applied to the turbine's rotor.

- The non-spinning or supplemental reserve is the extra generating capacity that is not currently connected to the system but which can be brought online after a short delay.

In addition, there are two other kinds of reserve power that are often discussed in combination with the operating reserve: the frequency-response reserve and the replacement reserve.

- The frequency-response reserve is provided as an automatic reaction to a loss in supply. It occurs because, immediately following a loss of supply, the generators slow down because of the increased load. To combat this slowing, many generators have a governor. By helping the generators to speed up, these governors provide a small boost to both the output frequency and the power of each generator. However, because the frequency-response reserve is often small and not at the discretion of the system operator, it is not considered part of the operating reserve.

- The replacement reserve (also known as contingency reserve) is reserve power provided by generators that require a longer startup time. It is used to relieve the generators providing the spinning or non-spinning reserve and thus restore the operating reserve.

The time periods over which all four kinds of reserve power operate are illustrated in Figure 19.

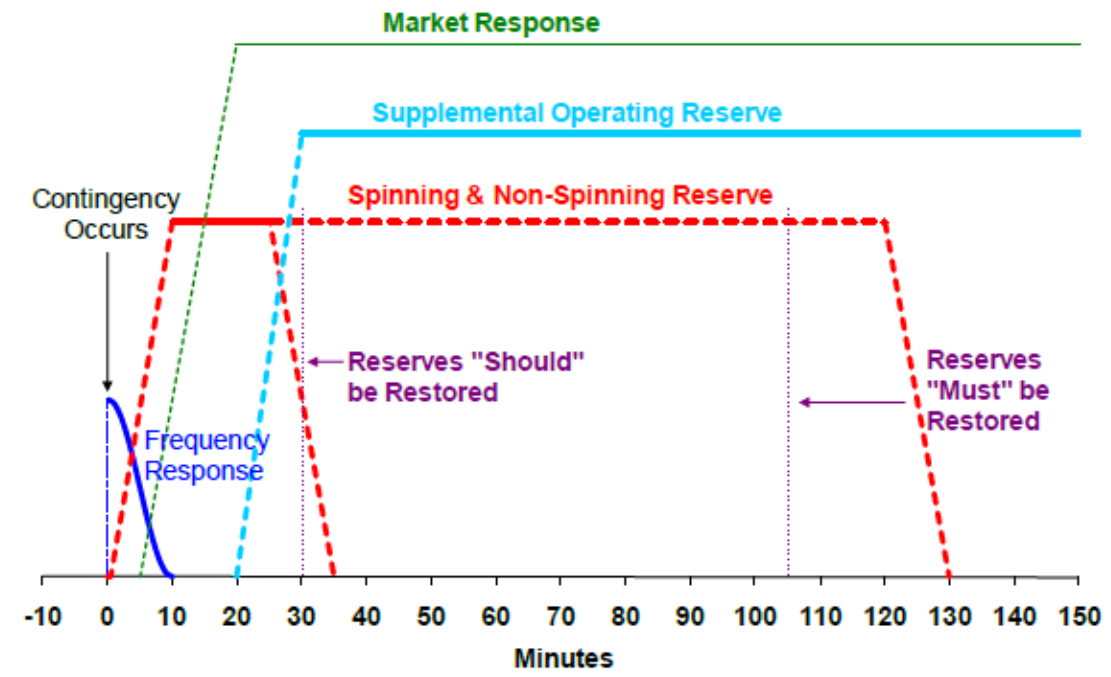

Figure 19. Reserve deployment as defined by NERC (Ela et al. 2010). ${ }^{65}$

${ }^{65}$ Reused by permission of IEEE. 


\subsubsection{WECC Standard BAL-STD-002-1 - Operating Reserves}

"The reliable operation of the interconnected power system requires that adequate generating capacity be available at all times to maintain scheduled frequency and avoid loss of firm load following transmission or generation contingencies. This generating capacity is necessary to:

- Supply requirements for load variations;

- Replace generating capacity and energy lost because of forced outages of generation or transmission equipment;

- Meet on-demand obligations; and

- Replace energy lost due to curtailment of interruptible imports" (WECC 2007).

\subsubsection{Minimum operating reserve requirements}

"Each Balancing Authority shall maintain minimum operating reserve, which is the sum of the following:

- Regulating reserve. Sufficient spinning reserve, immediately responsive to automatic generation control (AGC) to provide sufficient regulating margin to allow the Balancing Authority to meet NERC's control performance criteria.

- Contingency reserve. An amount of spinning reserve and non-spinning reserve (at least half of which must be spinning reserve), sufficient to meet the NERC disturbance control standard, equal to the greater of:

$0 \quad$ The loss of generating capacity due to forced outages of generation or transmission equipment that would result from the most severe single contingency; or

$0 \quad$ The sum of 5 percent of the load responsibility served by hydro generation and 7 percent of the load responsibility served by thermal generation.

The combined-unit ramp rate of each Balancing Authority's online, unloaded generating capacity must be capable of responding to the spinning reserve requirement of that Balancing Authority within 10 minutes.

- Additional reserve for interruptible imports. An amount of reserve, which can be made effective within 10 minutes, equal to interruptible imports.

- Additional reserve for on-demand obligations. An amount of reserve, which can be made effective within 10 minutes, equal to on-demand obligations to other entities or Balancing Authorities" (WECC 2007).

Acceptable types of non-spinning reserve:

- interruptible load;

- interruptible exports; 
- $\quad$ on-demand rights from other entities or Balancing Authorities;

- spinning reserve in excess of requirements; or

- offline generation, which qualifies as non-spinning reserve.

Knowledge of operating reserves shall be calculated such that the amount available (which can be fully activated in the next 10 minutes) will be known at all times (WECC 2007).

\subsubsection{Suggested changes to WECC operating reserves standard}

The Western Interconnection BAs discuss a "proposal by WECC to state that the minimum amount of contingency reserve should be the greater of the most severe single contingency or the sum of 3 percent of the balancing area load and 3 percent of the balancing area generation" (Ela et al. 2010).

\subsubsection{Impact of variable generation on reserves}

The increasing penetration of variable generation modifies several types of operating reserve requirements.

Power engineers in many countries are exploring the amount of additional reserve capacity and the corresponding costs when increasing the penetration of wind power. The investigations simulate system operation and analyze the effect of an increasing amount of wind power for different types of generation mix. The increase in short-term reserve requirement is estimated largely by statistical methods that combine the variability or forecast errors of wind power to that of load, and investigates the increase in the largest variations seen by the system. General conclusions on increasing the balancing requirement will depend on factors such as the region size, initial load variations and how concentrated/distributed wind power is sited (European Wind Energy Association 2010).

In 2006, an agreement on international cooperation was set up under the International Energy Agency (IEA) Task 25 to compare and analyze the outcome of different national power system studies. The 2009 report of this Task 25 (Holttinen et al. 2009) gives generalized conclusions based on studies from Denmark, Finland, Norway, Sweden, Germany, Ireland, Spain, Netherlands, Portugal, the United Kingdom and the U.S. The value of the combined assessment in the IEA Task 251 is that it allows the systematic relationship of the increased demand of system reserves to be shown as a function of wind energy penetration.

When considering the impacts of wind power on the different types of reserve requirements, it is of central importance to make a clear distinction between the need for flexibility in longer time scales of several hours to 1 day (power plants that can follow net load variation), and the need for reserves that can be activated in seconds or minutes (power plants that can follow unpredicted net load variations - demand minus wind).

Wind power development will have only a small influence on the amount of primary reserves needed. On time scales of seconds/minutes, rapid variations in total wind power capacity output occur randomly, as do existing load variations. When aggregated with load and generation variations, the increase in variability caused by wind is small. Furthermore, the amount of primary reserve allocated to the power systems is dominated by potential outages of large thermal generation plants, so it is more than large enough to cope with the rapid variations in wind. In practice, power plant generation is scheduled to match the anticipated trends in demand so that it can be balanced with the supply. For any deviations from the anticipated 
trends, primary and secondary reserves are operated continuously to keep system frequency close to its nominal value. In addition, wind power can provide its own primary reserve. On the time scale of 10 to 30 minutes, the impact of wind power on the need for secondary reserves will be significant and will increase due to wind energy penetration levels greater than 10 percent (IEA Wind 2011).

Wind power has a much more significant impact on the way conventional units are scheduled to follow the load (hour to day time-scales). In the absence of a perfect forecast, the unitcommitment decision will be surrounded by uncertainty, in addition to the normal uncertainty associated with load and conventional generation outage forecasting. The result is that sometimes a unit might be committed when it is not needed, and sometimes a unit might not be committed when it is needed. Here, the generation mix of the power system determines how the scheduling will change according to the expected wind power production - the more flexible power units there are, the later the unit commitment decisions need to be made (European Wind Energy Association 2010). Estimates for the increase in short-term reserve balancing capacities (Holttinen et al. 2009) show a wide range: 1 to 15 percent of installed wind power capacity at 10 percent penetration (of gross demand), and 4 to 18 percent of installed wind power capacity at 20 percent penetration.

Differences in the power system's operational routines explain how often the forecasts of load and wind are updated. If a redispatch based on a forecast update is done in 4 to 6 hours, this would lower the reserve requirements and costs of integrating wind, compared with scheduling based on only day-ahead forecasts. Emerging intra-day markets take this particularity into account by giving the opportunity for hourly updates. The way the power system is operated regarding the time lapse between forecast schedules and delivery has a decisive impact on the degree of uncertainty wind power will bring, and it indirectly will determine the amount of additional reserves required.

It is important to note that an increase in reserve requirements does not necessarily mean that new investments will have to be made; such as the construction of new thermal power plants. From an analysis of the system and from experience, it follows that the forecast uncertainty of incidental combinations of wind power generation and demand is critical for assessing the need for additional reserves, especially the "low demand, high wind" combination. Additional flexibility from conventional units is critical especially in situations of low load and high wind, because in such situations, the thermal plant may have to be ramped up fast because of sudden drops in wind power generation. More generally, increased wind power will mean conventional thermal units will have to be operated in a more flexible manner than if there were no wind energy (European Wind Energy Association 2010).

\subsubsection{Frequency response reserve ${ }^{66}$}

Frequency response provided by speed governors on conventional generators are required to provide a 5-percent speed droop characteristic. Additionally, at least some of these generators should have a sufficient capacity range for the actual deployment of their frequency response. This means they should be operated below their maximum and above their minimum capacity. In reality, even with the speed governor turned on and tuned for 5-percent speed droop, and with the sufficient upward and downward movement capacity, some generators do not provide the resulting 5-percent response. This depends on the technology, mode of operation, status of

\footnotetext{
${ }^{66}$ Contributed by Yuri Makarov, PNNL.
} 
the generator, set point control and other factors. The insufficient frequency response becomes a growing issue even without a significant presence of variable resources.

Variable renewable resources currently do not provide a frequency response from existing power plants. This means that with the increasing penetration of renewables, the actual frequency response will be decreasing even more. Insufficient frequency response can lead to balancing, control performance, and even reliability problems. This problem can be more significant during off-peak hours, when the system load reaches its minimum, while the variable generation is peaking (e.g., wind generation in California at night). In this situation, the relative displacement of conventional governor-equipped generation is increasing, and, consequently, the available frequency response in terms of the BA frequency bias.

The problem can be corrected by establishing a frequency response standard that requires each BA to provide a certain amount of frequency response in terms of capacity and deployment time.

\subsubsection{Regulation reserve ${ }^{67}$}

Multiple studies conducted on this topic in recent years suggest that the impact of variable generation on regulation reserves is very limited. This conclusion is confirmed by evidence from TSOs in Europe, coming from their practical experience with regulation requirements as they are exposed to large, absolute, or relative penetrations of renewable resources. These conclusions were made by German TSOs, Spain's Red Eléctrica de España (REE), Danish TSOs, and others.

On the other hand, there are studies that indicate a potential increase of regulation requirements with the increasing share of VG in the system. Such studies have been conducted by California ISO, KEMA Consulting, PNNL, and other organizations. Some grid operators have already found a correlation between the increasing VG capacity and regulation.

The reason for this difference in opinions needs to be further investigated. There are several factors that may contribute to this analysis:

1. The size of regulation reserve that has been historically procured. For instance, the regulation reserve in Spain is approximately $\pm 1,000 \mathrm{MW}$. In California, this reserve is historically somewhere in the range of $\pm 350-600 \mathrm{MW}$ (a system of approximately the same size).

2. Relaxed control performance standards in Europe compared to the U.S. control performance standard (CPS2).

3. Spain and Germany use fast-response hydro units for regulation, whereas California and electric reliability council of Texas (ERCOT) also employ slow, responsive, thermal resources. The use of slower resources increases the regulation capacity requirements.

4. VG resources in Germany, Spain and Denmark have a greater distributed nature compared with California, Pacific Northwest, and ERCOT. The distributed nature

\footnotetext{
${ }^{67}$ Contributed by Yuri Makarov, PNNL.
} 
decreases the relative impact of the variability and uncertainty of individual wind or solar resources.

5. Both Spanish and German TSOs employ accurate wind and solar forecast algorithms.

In the research area, the answer can be provided by a new generation of tools that mimic real scheduling, real-time dispatch processes, and regulation processes (including AGC models), and which reflect real forecast errors, impacts of variability, slow units' response, and other factors. These tools have been developed or are under development by General Electric Energy (GE), KEMA, National Renewable Energy Laboratory (NREL), PNNL, PLEXOS, and other research, consulting, and software manufacturing organizations.

\subsubsection{Load-following (tertiary) reserve}

There is a consensus in the research community and in the industry that VG impacts load following (real-time dispatch) capacity requirements. Those impacts can vary depending on the distribution of variable resources (e.g., resources concentrated in a small area have more impact), type of resources (e.g., solar resources may have less impact because of their ramps being opposite to the load ramps), wind and solar forecast errors, and other factors.

\subsubsection{Contingency reserve}

"Contingency reserve is required for the reliable operation of the interconnected power system. Adequate generating capacity must be available at all times to maintain scheduled frequency, and avoid loss of firm load following transmission or generation contingencies. This generating capacity is necessary to replace generating capacity and energy lost, due to forced outages of generation or transmission equipment" (NERC undated).

\subsubsection{VG tail events reserve}

"Variable generation tail event reserve: Reserves that are available to cover infrequent, but large ramps of variable generation. The requirements for such reserves are very similar to conventional contingency reserves in that response is only required infrequently. The difference is that large, variable generation ramping events are typically slower than conventional contingencies. While a conventional contingency happens instantly, a large, variable energy resource ramp will typically take 2 hours or longer for the full ramp. NERC reliability rules require contingency reserves to be restored within 90 minutes, making most variable generation tail events too slow to effectively use conventional contingency reserves. A reserve that is able to maintain response for 2 hours or longer may be required to respond to large, infrequent variable energy resource ramps" (NERC 2010a).

\subsubsection{Impact of new control performance standards on reserves}

The electric utility business faces a major transition to the new set of control performance standards (CPS). The most limiting CPS2 standard is now being replaced by more relaxed, new Balancing Authority's ACE limits (BAAL). This transition may have a profound impact on some types of operating reserves, and there are indications of this impact in some recent studies, as well as from the practical industry experience with their implementation.

Some recent studies indicate that the introduction of BAAL will result in reduced regulation requirements (Lu, N, Makarov, Y.V etc., 2010). Instead of being a continuous service, regulation may become an episodic service, needed only during certain hours of an operating day. The 
Bonneville Power Administration (BPA) already has observed this effect in its system, where the regulation was only applied during ramps.

The potential influence of the new CPS on other types of reserves needs further analyses.

European standards and practices are discussed in Appendix 20.

\subsection{Overgeneration ${ }^{68}$}

Overgeneration (aka, oversupply) occurs when the online generation exceeds demand, and there are difficulties in trying to reduce or uncommit the excess generation to a required level. In California and the Pacific Northwest, overgeneration happens during high water flows and high wind periods, when hydro and wind generation are peaking simultaneously, and system load is low. Overgeneration could cause multiple, negative impacts and consequences for the systems, including negative market prices, inadvertent energy accumulations, control performance standards compliance problems, frequency excursions, and ultimately could increase reliability threats. Many system operators are concerned about this problem, including BPA and California ISO. The range of solutions proposed so far is wide, but it still appears not to be adequate. The central questions that create a lot of discussion are whether the system operator has a right to curtail wind generation during overgeneration hours (hydro generation cannot be curtailed because of environmental restrictions); if other measures are not sufficient, whether wind farms are entitled to compensation; and which parties should pay the cost of overgeneration management. This section describes the current analyses on overgeneration conducted by California ISO and BPA, as well as some information detailing the European experience.

\subsubsection{CAISO analysis and practice}

"Overgeneration occurs when there is more generation and imports within a balancing area than load and exports can use. This situation develops after the system operator has exhausted all decremental energy bids available in the imbalance energy market, has pushed all regulating resources to the bottom of their operating range, and has exercised arrangements for out-ofmarket purchases for excess energy with neighboring Balancing Authorities" (CAISO 2011a).

"In California, overgeneration is most likely to occur under the confluence of some or all of the following conditions: light spring load conditions (historically with loads around 22,000 MW or less); all the nuclear plants online and at maximum production; hydro generation at high production levels due to snow melt in the mountains; long-start thermal units online, and operating at their minimum levels, because they are required for future operating hours; other generation in a regulatory "must take" status or required to be online for local reliability reasons; and wind generation at high production levels" (CAISO 2010a).

"The fundamental causes that precipitate an overgeneration condition are:

- A mismatch between scheduled generation and forecasted load;

- Managing must take generation during low load conditions;

- Load and resource forecast errors;

- More imports are scheduled than there is load on the system;

- Excess must take hydro generation and the need to avoid spilling water; and

${ }^{68}$ Provided by Yuri Makarov, PNNL

WESTERN ELECTRICITY COORDINATING COUNCIL - WW W. WECC.BIZ 155 NORTH 400 WEST • SUITE $200 \cdot S A L T$ LAKE CITY • UTAH • $84103-1114 \cdot$ PH 801.582 .0353 
- Excess unscheduled wind and solar generation" (CAISO 2011a).

In its 20 percent renewables penetration study, California ISO has made the following analysis: "The production simulations analyzed both a high hydro year (based on 2006 hydro production) and a low hydro year (based on 2007 hydro production), as well as sensitivities to assumptions about load growth and firm imports, to evaluate their effect on overgeneration. The maximum overgeneration occurred in a scenario that assumed no load growth between 2006 and 2012. The overgeneration in this case was approximately 0.3 percent (150 GWh) of annual renewable generation. Most of the overgeneration occurs in late spring (April-May), due to a combination of high generation from hydro and variable energy resources, and low loads. In general, overgeneration was found to be directly correlated to the amount of nondispatchable generation in the system. There appears to be sufficient dispatchable generation available to operate if the ISO is not prevented from doing so due to an excess of nondispatchable generation, including imports" (CAISO 2010a).

"Overgeneration was found to be directly correlated to the amount of nondispatchable generation in the system. Overgeneration, under the worst-case scenario, which assumes no load growth between 2006 and 2012, was 0.32 percent (150 GWh) of annual energy from renewable resources. There is potential to further relieve these instances of overgeneration by increasing the commitment of dispatchable resources in place of inflexible resources, such as firm imports" (CAISO 2010a).

"Figure 20 shows the simulated, five-minute, load-following, up-and-down capabilities from dispatchable generators for May 28, 2012. This is the capability of the dispatchable generators to move from one, 5-minute dispatch to the next. The figure shows adequate, 5-minute capability throughout the day... However, the figure shows low load-following down capability during the morning hours from 4 a.m. to 10 am.... The low load-following down capability is a direct consequence of the amount of dispatchable generation that is online. During the morning hours of May 28, 2012, ...very few dispatchable generators are online and most are already operating at or close to their minimum load point" (CAISO 2010a).

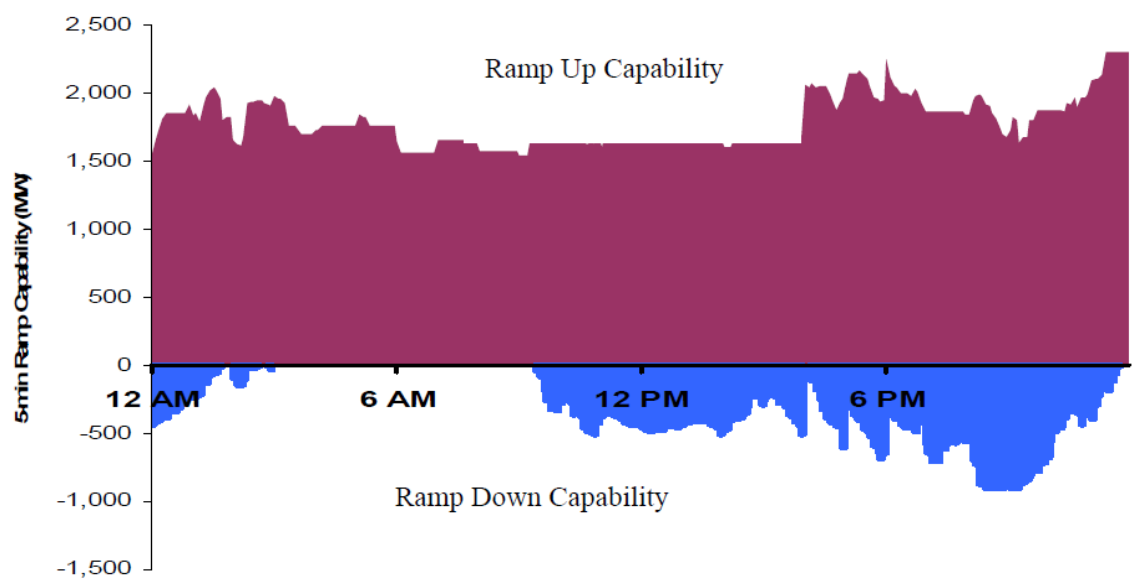

Figure 20. Simulated upward and downward 5-minute load following capability for May $28^{\text {th }}, 2012$ (CAISO 2010a). ${ }^{69}$

\footnotetext{
${ }^{69}$ Reused by permission of CAISO.
} 
"Figure 21 shows the overgeneration for May 28, 2012, obtained from the production simulation. This figure also shows the regulation down procurement (green line) and the CPS2 violation threshold (yellow line) for the same period. While there is significant, sustained overgeneration for a few hours from 5 a.m. to 8 a.m., the rest of the time, the overgeneration can be covered by the procured regulation or allowed to result in an Area Control Error (ACE) error if it is not sustained. Only large overgeneration sustained over 10 minutes may result in the curtailment of generation" (CAISO 2010a).

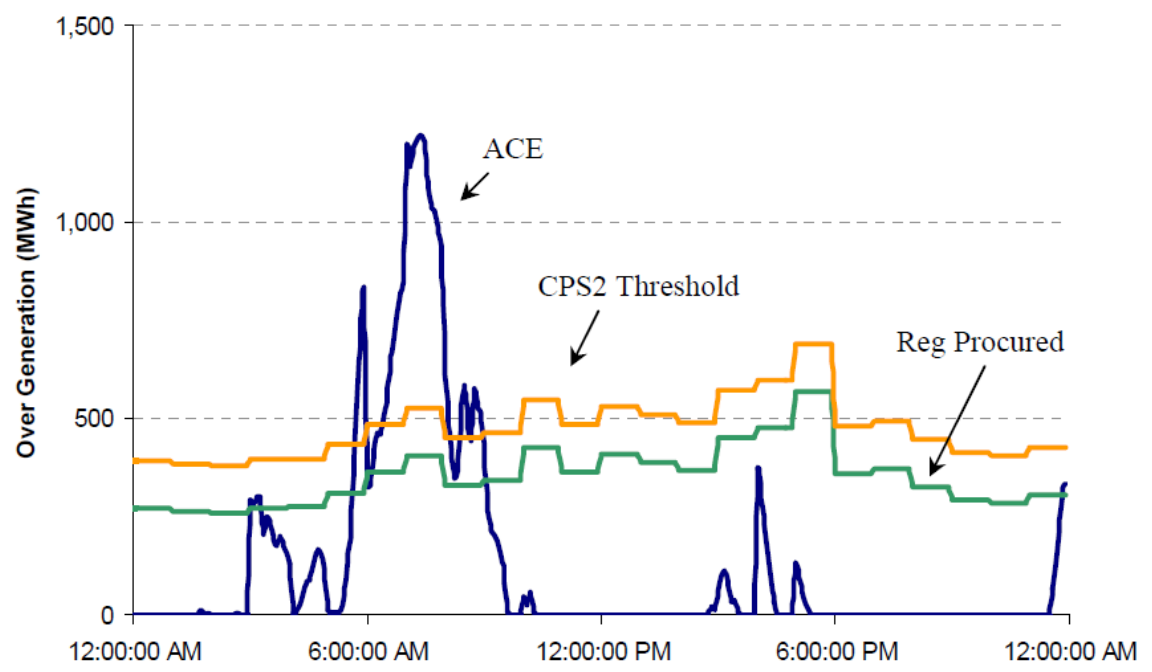

Figure 21. Detailed overgeneration analysis of May 28, 2012 (CAISO 2010a). ${ }^{70}$

"Figure 22 shows the relationship between overgeneration and the amount of dispatchable generation during the hours between 4 a.m. and 10 a.m. The traces show that there is a direct correlation between overgeneration and lack of dispatchable generation. When the dispatchable generation is approaching zero, overgeneration is high. Under these conditions, with very little dispatchable generation online, the fast ramp in solar generation results in an overgeneration condition. It should be noted that the solar generation ramp is not the cause of the overgeneration, rather it's the trigger. The cause for overgeneration is the lack of flexible or dispatchable resources during these hours" (CAISO 2010a).

\footnotetext{
${ }^{70}$ Reused by permission of CAISO.
} 


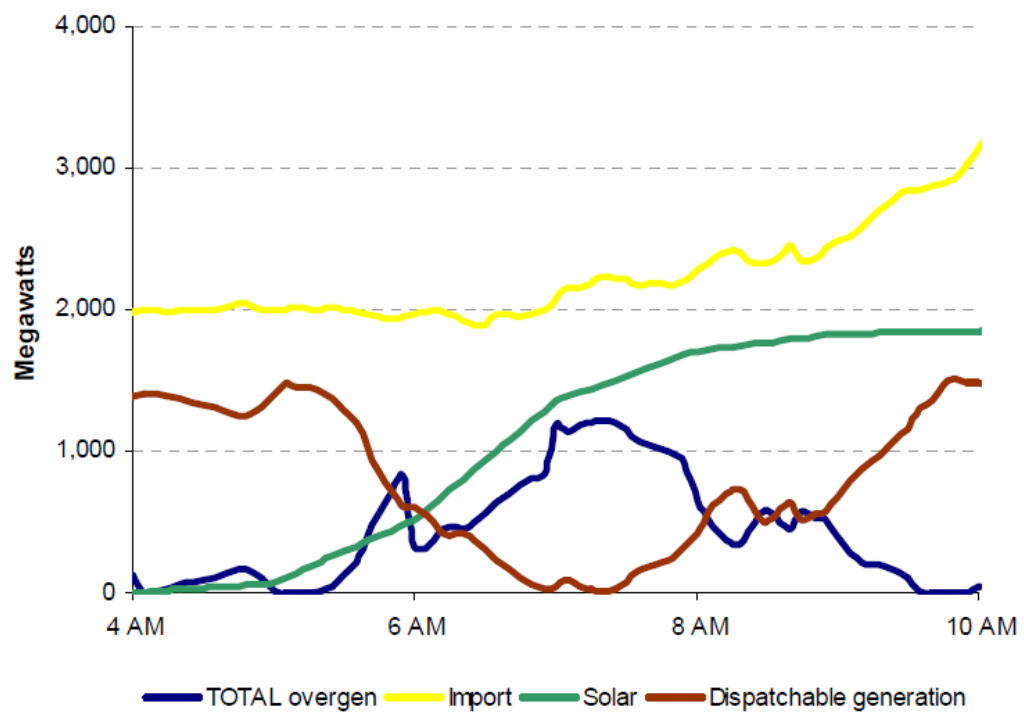

Figure 22. Dispatchable generation and overgeneration (CAISO 2010a). ${ }^{71}$

In the initial straw proposal, "Renewables Integration Vision and Roadmap," California ISO proposes the following course of actions for overloads: "When anticipating overgeneration conditions, ISO operators will send out a market notice to request additional, decremental bids. If insufficient decremental bids are received from scheduling coordinators, and the area control error can no longer be maintained within acceptable limits, ISO operators could declare a system emergency" (CAISO 2011a).

\subsubsection{Proposed oversupply management at BPA}

"High flows on the Columbia-Snake River system create the potential for increased total dissolved gas (TDG) levels. Spilling too much water over the dams creates excessive TDG, which can lead to gas bubble trauma in fish and other aquatic organisms. Putting water through turbines to generate electricity does not increase the TDG levels in the river. However, there must be load to absorb that power.

"In addition, if all hydro turbines are operating at maximum capacity, then BPA has no choice but to spill - this is called 'lack-of-turbine spill.' High, seasonal river flows in the Pacific Northwest occasionally generate more hydropower than needed to meet export sales and regional demand.

"The chance of high flows occurring in any one year, and lasting for a month or more, is one in three. In these conditions, the market is flooded with low cost hydroelectricity. This creates an economic incentive for regional utilities and independent power producers to displace thermal generation, replacing it with power from the market. That is not the case with variable energy resources (VERs) that receive production tax credits (PTCs) and renewable energy credits (RECs) for generation from their specific generators. Once all displaceable generation in the Balancing Authority is displaced, but there is still available turbine capacity, additional spill is considered 'lack-of-market spill.'

\footnotetext{
${ }^{71}$ Reused by permission of CAISO.
} 
"BPA's effort to control TDG by displacing generation within our Balancing Authority, in order to reduce lack-of-market spill, is the foundation of BPA's oversupply policy" (BPA 2012).

\subsubsection{BPA oversupply analysis modeling (BPA 2012)}

BPA refined its oversupply analysis modeling based on its Spring 2011 experience. The refined modeling, using forecasted load and resource information for 2012, were then combined with 70 water years (1929 -1998), 15 hydro shapes (1996-2010), weekly HLH/LLH shapes, and 30 synthetic wind generation patterns consistent with those used in BPA rate case. The above combinations resulted in 31,500 games or potential outcomes (BPA 2012)

"BPA's oversupply modeling estimated the amount of displacement we might expect in 2012 using our forecast of the size of the wind fleet and loads. Modeling of a large number of different water and wind conditions resulted in an average estimate of $414 \mathrm{MW}$-months (302,000 MWh), about three times what occurred in $2011 \ldots$... This amount is approximately 3 to 4 percent of total wind generation expected in 2012. Based upon preliminary displacement cost data, provided by a portion of the wind fleet, the expected value of lost contract revenue, PTCs (29 percent of wind fleet), and RECs (valued at $\$ 16 / \mathrm{MWh}$ ) of this amount of curtailment is estimated to be $\$ 12$ million" (BPA 2012).

Figure 23 shows BPA overgeneration forecast for 2012. There is a 35 percent probability that in 2012 , conditions will not result in oversupply conditions. There is a low probability that extreme conditions could lead to significantly greater amounts of oversupply than experienced in 2011 (BPA 2012).

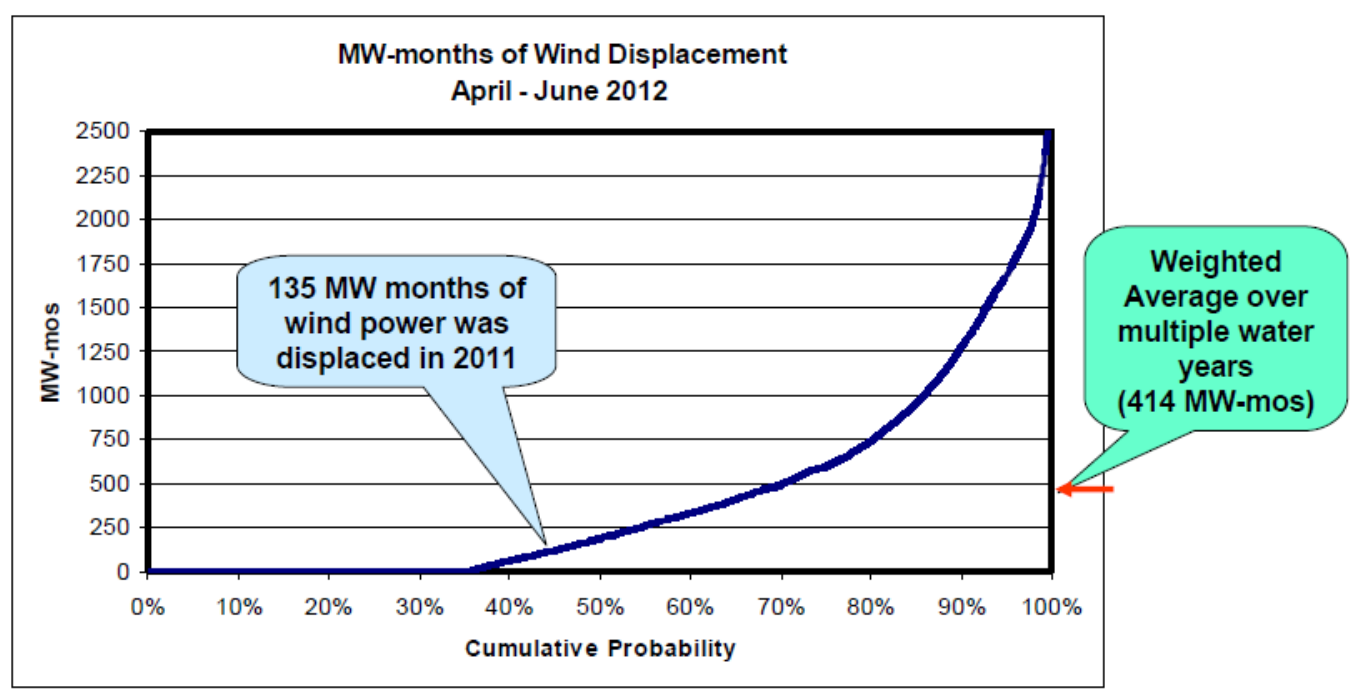

Figure 23. BPA overgeneration forecast for 2012 (BPA 2012). ${ }^{72}$

\subsubsection{Planning solutions considered by BPA (BPA 2011)}

BPA-proposed solutions aim to facilitate exports and access to loads.

\section{Transmission utilization (BPA 2011)}

\footnotetext{
${ }^{72}$ Reused by permission of BPA.
} 
- Improve use factor of transmission and interties. BPA analysis showed a high level of utilization. The AC intertie was scheduled up to the operating transfer capability (OTC) limit, in most cases, when the Northwest is experiencing high levels of hydro runoff (BPA 2011).

- $\quad$ Strive for more advance coordination on the release of transmission rights at CAISO (90 minutes before the delivery hour) and BPA (50 minutes before the delivery hour) (BPA 2011).

- Examine algorithms and protocols for assigning transmission to avoid inadvertent flow seizing intertie. The inadvertent flow on the California-Oregon Intertie (COI) is managed with the unscheduled loop flow mitigation procedure. During times of COI congestion, if BPA determines inadvertent flow is negatively impacting COl's system operating limit (SOL), BPA will ask CAISO to implement the procedure (BPA 2011).

\section{Outage coordination (BPA 2011)}

- Modify scheduled maintenance that would limit export capabilities. June 2010 events increased awareness of new concerns during spring runoff, which requires increased coordination and communication on both internal and external BPA outages.

- Further coordinate outages with other utilities to modify planned maintenance to improve intertie capacity. Outages by external entities that create reliability concerns can be modified by the WECC Reliability Coordinator.

- Issue a "Restricted Maintenance Operations" declaration for transmission maintenance during high runoff.

Evaluate ability to schedule to the east (BPA 2011)

This would require action on two elements:

1. Adding scheduling points at Summer Lake to move power east. The required facilities have not been constructed, and the operating studies and operating procedures must be completed. The loading of Summer Lake east bound would require a heavy flow on COI. The COI path loading is close to a 1 to 1 impact on flow east bound from Summer Lake to Idaho. Idaho Power and PacifiCorp are proposing system-to-system scheduling to mitigate any potential impact.

2. Adding infrastructure from John Day to Summer Lake to increase operating transfer capability (OTC). Transmission south is already fully utilized and scheduling east would impact the OTC south bound (BPA 2011).

\section{Seek more flexibility on use of Canadian storage (BPA 2011)}

BPA regularly coordinates storage agreements with Canada that are mutually beneficial. BPA will coordinate with B.C. Hydro on any possible reduction in flows at Arrow Dam during these high-runoff events (BPA 2011).

\section{Reduce reserves for wind (BPA 2011)}

These are standard actions taken by BPA and U.S. Army Corp of Engineers (COE) to minimize system total dissolved gas (TDG). BPA will reduce incremental capacities (INCs) as well as decremental capacities (DECs) if studies indicate that the reduction can effectively reduce TDG. 
However, there will always be sufficient INC and DEC capacity made available for load balancing (BPA 2011).

\section{Additional or time-shifted irrigation pump load (BPA 2011)}

There were too many uncertainties to work out. Customers believed that the benefits to themselves and to growers were insufficient to alter historical behavior. It was not clear that irrigation pump loads could be continuously available during the overgeneration period. In late June and July, many irrigators may be pumping around the clock. Earlier in the season, seedlings may require consistent irrigation during windy conditions. Many large irrigators may already have shifted irrigation times to light load hours (LLH) where it makes economic sense to do so (BPA 2011).

\section{Long-term actions (BPA 2011)}

These are long-term actions requiring two years or more to implement:

- $\quad$ Automating remedial action schemes (RAS). BPA currently is evaluating RAS automation for better operation of the system and not from the perspective of increasing capacity. Transmission planning will addresses this issue as part of RAS' asset management program.

- Add infrastructure or make other physical improvements (including COI). Facilities work is underway (shunt and series capacitors) to increase available COI capacity to its full 4,800-MW rating.

- Improve north-to-south intertie efficiency. BPA had suggested that installing 400- to 500Mvar SVC at Captain Jack most likely will eliminate the need for a Klamath Falls generation role in the COI rating (BPA 2011).

\subsubsection{Suggested operational practices (BPA 2012)}

Suggested operational practices are listed below.

\section{Environmental redispatch (BPA 2012)}

In 2011, "BPA temporarily limited the output of wind generation and replaced energy output with free Federal hydro generation, in order to maximize the amount of water put through turbines. Environmental redispatch (ER) was used to control total dissolved gas (TDG) levels May 18 July 10 , mostly during LLH. Almost all thermal generation was already shut down during LLHs, due to the availability of low- or zero-priced hydropower below their marginal production costs. Approximately 4,700 MWh of non-variable energy resource energy was redispatched. Approximately 97,500 MWh (135 MW months) of wind energy was redispatched. This was approximately 1.2 percent of total wind generation over the previous 12 months" (BPA 2012),

\section{Oversupply management protocol}

"Prior to implementing least-cost displacement, BPA will employ all reasonable strategies available for managing TDG levels. If reasonable operational actions are not sufficient to manage TDG, when the levels measured by the U.S. Army Corps of Engineers exceed Oregon and Washington water quality standards at projects that are spilling past unloaded turbines, then BPA will reduce any thermal generation in our Balancing Authority Area (BAA) to minimum output levels and replace wind schedules with free Federal Columbia River Power System (FCRPS) energy - as well as pay wind generators based on the least cost displacement curve 
to reduce generation in our BAA. In its initial proposal in the rate case, BPA will propose a roughly 50/50 cost allocation between users of the Federal Base System and wind generators" (BPA 2012).

\section{Least-cost displacement protocol (BPA 2012)}

"BPA will displace non-federal generation in BPA's BAA to mitigate TDG levels. There will two types of displacement - non-VER and VER.

Non-VER. Declare minimum generation levels consistent with: ramp capability, plant reliability and safety, and commitment to providing local VAr support or operating reserves. During TDG events, thermal plants will be displaced down to declared minimum levels and their hourly schedules replaced with free FCRPS generation. BPA is working with Energy Northwest and the broader nuclear industry to determine what is an appropriate, minimum generation level for the Columbia Generating Station.

VERs and Non-VERs that qualify for RECs and PTCS. BPA will displace non-federal VER generation in BPA's BAA to mitigate TDG levels. Displaced VER generation will receive compensation based on audited costs. VER generators will submit \$/MWh costs annually.... Eligible costs include unbundled RECs, PTCs, and the contract value of the bundled sale and purchase of RECs and metered generation. Compensation will be calculated by multiplying the \$/MWh by the difference in MWh between scheduled generation and the level that BPA dispatchers direct the generator to achieve" (BPA 2012).

\subsubsection{Future generation interconnections (BPA 2012)}

"Generation interconnections that have REC or PTCs that enter commercial operation after March 6, 2012, have two options:

1. Choose to be redispatched without compensation and therefore not share in the cost allocation; and.

2. Choose partial compensation, which will be set at a figure up to 50 percent, and share in the proposed cost allocation" (BPA 2012).

\subsubsection{BPA actions prior to displacement (BPA 2012)}

BPA will employ reasonable operational means available to avoid thermal and VER displacement, while still meeting all system non-power requirements such as flood control. These may include:

- Spill swaps;

- Implementing spill because of lack-of-demand:

- Position Banks Lake to have the maximum amount of pump load available;

- Delay generator and transmission maintenance outages and capital work in May, June, and July when feasible; and

- $\quad$ Reduce reserves and others (BPA 2012).

International experience in wind and solar energy sources are presented in Appendix 21. 


\section{Modeling Using VG Specific Models}

This section addresses the models developed by modeling and validation work group, and the Renewable Energy Modeling Task Force(REMTF).

\subsection{Models developed by Modeling and Validation Work Group and REMTF $^{73}$}

"Historically, most dynamic models for wind turbine generators (WTG) have been developed by manufacturers and software vendors as proprietary, user-written models. These models have generally been distributed as object files only readable by software, instead of source code that can be reviewed by users. Incorporating user-written models into the network model of large interconnected systems can present several challenges:

- Difficult to certify quality assurance: With models developed by individual users and companies, it is difficult to investigate whether the model development and resulting code meets a satisfactory level of quality assurance.

- Difficult to gain insight on model behavior: Beyond analyzing model behavior through simulation results, it is sometimes necessary to follow the source code. Object files are the outcome of the original source code being compiled, and are not viewable by users - becoming a "black box" with contents hidden from the user. Thus, it is a challenge for model users to better understand the model behavior.

- Difficult to maintain: To comply with multiple versions of different software, it is necessary to maintain numerous versions of the same model, and model maintenance procedures become demanding" (Lin 2012).

To resolve these issues, WECC, through its Wind Generator Modeling Group (WGMG), has led a comprehensive effort to develop generic WTG dynamic models suitable for system analysis (Lin 2012, Asmine et al. 2011, Ellis et al. 2011).

The generic models satisfy the following requirements:

- Generic models are tunable to parameters to represent different WTGs of the same "type" (not specific to a particular manufacturer or model of WTG).

- They are nonproprietary (model and data can be shared).

- They form a standard library (not user-written).

- They are cross-platform compatible.

- They are fully documented block diagrams and descriptions, and default parameter sets.

- They are validated against field tests, factory tests, or against electromagnetic transient models that have themselves been validated against test data (Ellis et al. 2011).

The IEEE Working Group on Dynamic Performance of Wind Power Generation has also been promoting adequate WTG models for bulk power system simulations. The generic dynamic

\footnotetext{
${ }^{73}$ Contributed by Pengwei Du and Yuri Makarov, PNNL.
} 
models for four major WTG topologies have been developed for the simulation of events in typical transient stability analysis, such as 20 to 30 seconds, as shown in Figure 24 (Lin 2012, Asmine et al. 2011).

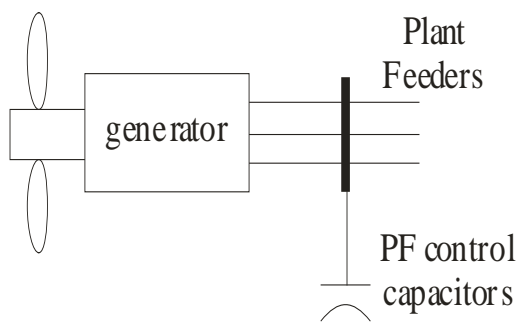

Type 1 - Conventional induction generator.

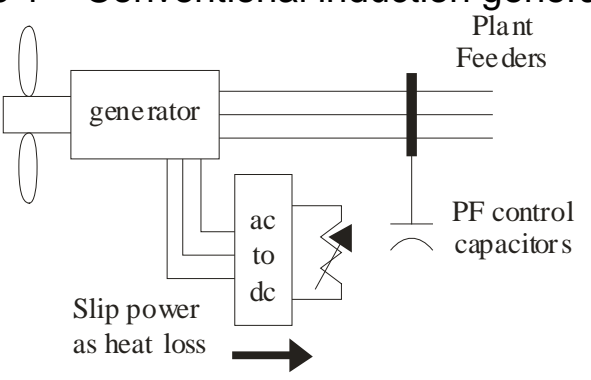

Type 2 - Wound rotor induction generator with variable rotor resistance.

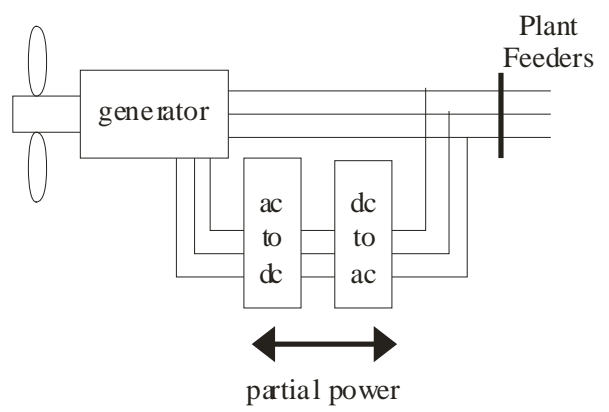

Type 3 - Doubly-fed induction generator.

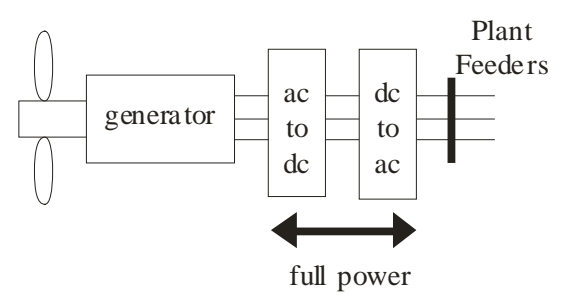

Type 4 - Full converter interface.

Figure 24. Four major WTG topologies and their generic models (Ellis et al. 2011). ${ }^{74}$

WECC has produced an initial set of generic models available in PSSE, ${ }^{75} \mathrm{PSLF}^{76}$ and other platforms as library models (Ellis et al. 2011).

\footnotetext{
${ }^{74}$ Reused by permission of IEEE.

${ }^{75} \mathrm{PSS} / \mathrm{E}$ is the premier software tool used by electrical transmission participants worldwide.

${ }^{76}$ PSLF is a software tool that enables power system planners to perform AC and DC steady-state power flow and
} 
In all models, voltage and frequency protection are modeled separately (Table 4) (Ellis et al. 2011).

Table 4. Generic WTG models available in PSSE and PSLF (Ellis et al. 2011) ${ }^{77}$

\begin{tabular}{|c|c|c|c|c|c|}
\hline \multirow[t]{5}{*}{ PSLF } & Model Type & Type 1 & Type 2 & Type 3 & Type 4 \\
\hline & Generator & wt1g & wt2g & wt $3 g$ & wt4g \\
\hline & Excitation / Controller & & wt2e & wt3e & wt4e \\
\hline & Turbine & wt1t & wt2t & wt3t & \\
\hline & Pitch Controller/Pseudo Gov. & wt1p & wt2p & wt3p & \\
\hline \multirow[t]{6}{*}{ PSSE } & Generic model & WT1 & WT2 & WT3 & WT4 \\
\hline & Generator & WT1G & WT2G & WT3G & WT4G \\
\hline & El. Controller & & WT2E & WT3E & WT4E \\
\hline & Turbine/shaft & WT12T & WT12T & WT3T & \\
\hline & Pitch control & & & WT3P & \\
\hline & Pseudo Gov/: aerodynamics & WT12A & WT12A & & \\
\hline
\end{tabular}

dynamics analysis.

${ }^{77}$ Reused by permission of IEEE. 


\section{VG Grid Interconnection Considerations}

This section addresses local VG interconnection issues and requirements.

\subsection{Wind generation interconnection studies ${ }^{78}$}

These studies address all major interconnection issues from wind generators to the point of interconnection. They can include:

- Load flow study

- Short-circuit study

- Harmonic study

- Insulation coordination study

- Dynamic and transient stability study

- Grounding and neutral grounding study

- Electromagnetic interference study

- Voltage fluctuation study (Martin 2010).

\subsection{FERC Order 661- $A^{79}$}

FERC Order 661-A (FERC 2005) establishes the requirements for large wind plants seeking to interconnect to the grid. Almost all regional coordinating councils are respecting this ruling and have adopted similar reliability guidelines.

FERC Order 661-A would require large wind plants seeking to interconnect to the grid to:

- Demonstrate low-voltage, ride-through capability; in other words, show that the plant can remain online during voltage disturbances, up to specified time periods and associated voltage levels;

- Have supervisory control and data acquisition (SCADA) capability to transmit data and receive instructions from the transmission provider; and

- Maintain a power factor within the range of 0.95 leading to 0.95 lagging, measured at the high-voltage side of the substation transformers.

The interconnection customer shall not disable power factor equipment while the wind plant is in operation. Wind plants also shall be able to provide sufficient dynamic voltage support in lieu of the power system stabilizer and automatic voltage regulation at the generator excitation system, if the system impact study shows that this is required for system safety or reliability.

The following sections outline similar guidelines as adopted by different organizations. These guidelines are relevant to planning, operation and control, and/or market operation.

\footnotetext{
${ }^{78}$ Contributed by Yuri Makarov, PNNL.

${ }^{79}$ Contributed by Hassan Ghoudjehbaklou, SDG\&E.
} 


\subsection{Ride-through capability ${ }^{80}$}

This section considers voltage and frequency ride-through issues and standards.

\subsubsection{Voltage ride-through capability}

"The ride-through capabilities required are (a) high- and low-voltage ride-through, and (b) highand low-frequency ride-through" (CAISO 2010b).

\subsubsection{Reliability need for voltage ride-through capability}

"The interconnection of new generators should not introduce adverse operating impacts to the existing transmission system. Sympathetic tripping of wind plants and solar facilities is a known issue for faults near generating stations. Immediately after a fault occurs, the voltage will typically collapse on the faulted phase or phases. Immediately after the fault is cleared, the system voltage may experience a transient high-voltage condition. This high-voltage condition near generating plants may be magnified due to the high level of shunt capacitors that are installed within the generating plant for power factor correction or voltage control.

Typically, most transmission system faults will be cleared within several cycles. However, if new generation facilities are not designed with ride-through capability to withstand the temporary low- and high-voltage conditions during the fault inception and clearing periods, then generation facilities will trip and stay offline even after the fault is cleared. The result is that generation may be lost for faults on the transmission system, even if the fault was temporary and successfully cleared.

High- and low-voltage ride-through capability will ensure that generators stay in service and connected to the grid during transient voltage conditions, and during system fault inception and clearing. WECC policy states that a control area operator should be able to withstand the loss of the largest generator by procuring sufficient spinning reserves. One consequence of regularly losing all or part of the generation, due to sympathetic tripping from the outage of transmission lines or other generators, is the adverse impact on control-area performance. A fault that trips a nearby generation unit, plus a significant amount of wind or solar generation (via sympathetic tripping), would result in a more-severe system imbalance on the control area. This could increase the magnitude of the largest single contingency, which has both reliability and financial implications."

“California ISO's discussions with several solar photovoltaic (PV) project developers indicate that, while not required, most utility-scale generation projects are utilizing inverters that have controls typically designed for small-scale distributed energy resources of 10 MVA or less. These inverters are designed to meet codes and standards per Underwriters Laboratories (UL) 1741 and IEEE 1547. The IEEE 1547 standard prescribes certain voltage and frequency trip settings, which are intended to trip the generation plant offline soon after the transient voltage and/or frequency condition is observed. Also, once the generation plant is tripped offline, it is required to stay offline for 5 minutes after the fault. This presents a fundamental conflict. While the voltage and frequency ride-through capabilities are needed from generation plants so the plant can stay connected to the grid, the default voltage and frequency settings for inverters are such that the plant trips offline shortly after the fault."

\footnotetext{
${ }^{80}$ Contributed by Yuri Makarov, PNNL.
} 
"The impact of using IEEE 1547 standard control settings generation projects was analyzed in a recent IEEE paper (Bebic et al. 2009), which concluded:

'With substantial PV generation that is compliant with IEEE 1547, system reliability is considerably reduced because of the extensive loss of PV generation during transmission faults. Adding low-voltage ride-through capability to PV systems improves the reliability of transmission systems with high-penetration PV'" (CAISO 2010b).

\subsubsection{Voltage ride-through capability standards}

"Presently, a few voltage ride-through standards exist (FERC 2005). FERC Order No. 661a describes the standard for wind generators. Within United States, a regional standard is under development - NERC PRC-024-1 standard" (NERC 2010c, CAISO 2010b).

“NERC-PRC-024-1 standard 'Generator Performance During Frequency and Voltage Excursion' is part of NERC's 'Project 2007-09: Generator Verification.' The intent of this standard is to establish technology-neutral requirements for all generators concerning voltage and frequency events. Requirements are currently being drafted for high/low-voltage and high/low-frequency ride-through capabilities."

"Table 5 lists high/low-voltage limit and time requirements, as currently prescribed by the draft 1 of NERC PRC-024-1 standard" (NERC 2009).

Table 5. NERC PRC-024-1 voltage ride-through performance requirement (CAISO 2010b) ${ }^{81}$

\begin{tabular}{|c|c|c|c|}
\hline \multicolumn{2}{|c|}{ HVRT DURATION } & \multicolumn{2}{|c|}{ LVRT DURATION } \\
\hline Time (Sec) & Voltage (p.u.) & Time (Sec) & Voltage (p.u.) \\
\hline 0.20 & 1.200 & 0.15 & 0.000 \\
\hline 0.50 & 1.175 & 0.30 & 0.450 \\
\hline 1.00 & 1.150 & 2.00 & 0.650 \\
\hline 4.00 & 1.100 & 3.00 & 0.750 \\
\hline & & 4.00 & 0.900 \\
\hline
\end{tabular}

\subsubsection{Frequency ride-through capability}

This section describes some details about the reliability need for frequency ride-through capability and its standards.

\subsubsection{Reliability need for frequency ride-through capability}

"The frequency on the power system is related to the amount of load and generation that is connected. When the load and generation are precisely balanced, the frequency will be $60 \mathrm{~Hz}$. In the event that generation is lost through an unplanned or forced outage (e.g., a generating unit trips offline), the frequency will deviate from the nominal of $60 \mathrm{~Hz}$. Immediately following the disturbance, the governors on the remaining generation units will adjust to attempt to arrest the frequency decline. It may be necessary for the AGC to make adjustments to bring the system

\footnotetext{
${ }^{81}$ Reused by permission of CAISO.
} 
frequency back to $60 \mathrm{~Hz}$. During this transition time, it is essential for the system generators to remain online. If additional generators trip during the transition, the system frequency will continue to deteriorate, and frequency restoration will be more difficult" (CAISO 2010b).

\subsubsection{Frequency ride-through capability standards}

"Article 9.7.3 of Large Generation Interconnection Agreement (LGIA) establishes the need for interconnection customers to design high- and low-frequency ride-through, as required by WECC. WECC's frequency ride-through requirements are set forth in the WECC Off-Nominal Frequency (ONF) Load Shedding and Restoration Plan (WECC 2010). The frequency ridethrough requirements contained in the latest draft of NERC PRC-024-1 conflict with frequency ride-through requirements contained in the WECC ONF Plan" (CAISO 2010b).

"Table 6 below lists under/over frequency limits and WECC minimum times as per the WECC ONF Plan."

Table 6. WECC generator ONF performance requirement (WECC 2010)

\begin{tabular}{|c|c|c|}
\hline $\begin{array}{c}\text { Under-frequency } \\
\text { Limit }\end{array}$ & $\begin{array}{c}\text { Over-frequency } \\
\text { Limit }\end{array}$ & $\begin{array}{c}\text { WECC Minimum } \\
\text { Time }\end{array}$ \\
\hline$>59.4 \mathrm{~Hz}$ & $60 \mathrm{~Hz}$ to $<60.6 \mathrm{~Hz}$ & $\begin{array}{l}\text { N/A (continuous } \\
\text { operation) }\end{array}$ \\
\hline$\leq 59.4 \mathrm{~Hz}$ & $\geq 60.6 \mathrm{~Hz}$ & 3 minutes \\
\hline$\leq 58.4 \mathrm{~Hz}$ & $\geq 61.6 \mathrm{~Hz}$ & 30 seconds \\
\hline$\leq 57.8 \mathrm{~Hz}$ & - & 7.5 seconds \\
\hline$\leq 57.3 \mathrm{~Hz}$ & - & 45 cycles \\
\hline$\leq 57 \mathrm{~Hz}$ & $>61.7 \mathrm{~Hz}$ & Instantaneous trip \\
\hline
\end{tabular}

\subsection{Voltage support and power factor ${ }^{82}$}

Transmission networks require reactive power support to transport real power over long distances, while maintaining system voltage within acceptable limits. Insufficient reactive power at critical buses of the network can result in poor voltage profile and even voltage collapse. Reactive power needs to be provided locally because reactive power losses over long distances are substantial.

"Why should we want to transmit reactive power anyway? Is it not just a troublesome concept, invented by the theoreticians, that is best disregarded? The answer is that reactive power is consumed not only by most of the network elements, but also by most of the consumer loads, so it must be supplied somewhere. If we can't transmit it very easily, then it ought to be generated where it is needed" (Kalsi et al. 2003).

Fixed capacitor banks are commonly used to provide voltage supports. However, because the reactive power generated by capacitors is proportional to $V^{2}$, they provide less reactive power when reactive power is needed the most, such as during a voltage collapse, limiting their applications.

\footnotetext{
${ }^{82}$ Contributed by Hassan Ghoudjehbaklou, SDG\&E, and David Tovar, EPE.
} 
The system increasingly will require fast-response, dynamic, voltage-control devices to allow voltage recovery after contingencies, while preventing excess voltages before the contingencies. These devices also provide continuous voltage support and improved voltage regulation. Some of the devices that provide dynamic and continuous voltage support are static compensators (STATCOMs) and static VAr compensators (SVCs). These devices use power electronics that can adjust power and voltage outputs almost instantaneously (ISO/RTO Council Planning Committee 2006).

The following lists some of the controls that usually are included in a voltage support scheme, for both pre-contingency and post-contingency cases:

- Shunt capacitors status (On/Off)

- Shunt reactors (On/Off)

- Regulated bus set points (volts)

- LTC (load tap changers)

- STATCOM, SVC, or other VAr supporting FACT

- Line or transformer status (Open/Close)

- Units (Mvar)

- Units (MW) to be able to get Mvar support.

The operational constraints include:

- Bus voltage limits (high and low)

- Area reactive reserve requirement

- Unit reactive limits

- Shunt reactive capacity limits

- FACT device limits

- Maximum and minimum tap positions.

Also, some reports mention other innovative voltage support devices, which might provide superior dynamic performances, if utilized effectively. A comprehensive list of these new devices is outside the scope of this guideline. (Interested readers are encouraged to research dynamic superconducting condensers or other new technologies, as examples.) In the following sections, available guidelines, requirements, and regulations that are concerned with voltage support and power factor requirements, mainly for renewable sources, will be investigated.

Appendix 22 presents the impacts of VG on inertia and frequency response.

\subsubsection{Discussion on the role of frequency response}

"The tightly regulated frequency of power system is critical to ensure reliability. Large-frequency excursions may lead to an overstress of power plants, or trigger the actions of under- or overfrequency relays. Under-frequency load shedding involves interrupting electric service to large, 
pre-set groups of customers; these customers will experience a blackout. Shedding large amounts of load in this manner is a drastic action (because customers' electric service is interrupted) that can have unintended consequences (because it may lead to an even wider spread blackout)" (Eto et al. 2010).

"The control of power system frequency is mainly through adjustments to the output of generators. The actions performed by generators for frequency control can be classified into three categories based on their response timeframe:

1. Primary frequency control involves the autonomous, automatic, and rapid action (i.e., within seconds) of a generator (and specific types of demand response) to change its output to oppose a change in frequency;

2. Secondary frequency control involves slower, centrally (i.e., externally) directed actions that affect frequency more slowly than primary control as part of automatic generation control (i.e., in tens of seconds to minutes); and

3. Tertiary frequency control refers to centrally coordinated actions (i.e., it is a form of what we have called secondary frequency control) that operate on an even longer time scale (i.e., minutes to tens of minutes) than primary frequency control and secondary frequency control provided through AGC" (Miller et al. 2010, Eto et al. 2010).

"Ensuring reliability requires the continuous availability of a critical component of operating reserves called primary frequency control reserves. These reserves are normally provided by generating units that are online and operating below their full generating capability. Primary frequency response is the capabilities (i.e., megawatt magnitude, speed of delivery and sustainability) that can be deployed from the unit to provide acceptable frequency responses formation of a frequency nadir and the settling frequency - to ensure reliability following a sudden loss of generation. Magnitude, speed of deployment, sustainability, and deliverability are critical aspects of primary frequency control" (Eto et al. 2010).

\subsubsection{Sources of primary frequency response}

“The responses from machines' governors are the key to maintaining frequency to within acceptable range by providing primary frequency control. In response to a frequency deviation, generators withdraw or inject power into the system to help maintain generation and load balance to maintain scheduled frequency within the desired range" (Eto et al. 2010).

"On the other hand, provision of primary frequency control can be supplemental by other resources as described below:

- Demand response (paying loads to turn off) was found to be effective and was less expensive. Expanded use of demand response (potentially including smart grid applications), starting with broader industry appreciation of the role of demand response in augmenting primary and secondary frequency control reserves;

- Expanded use of frequency control capabilities that could be provided by variable renewable generation technologies (primary frequency control, etc.); and

- Expanded use of advanced technologies, such as energy storage and electric vehicles" (Eto et al. 2010). 


\subsubsection{Calculation of frequency response from simulations or experimental data ${ }^{83}$}

In the study led by the Lawrence Berkeley National Laboratory (LBNL), frequency responses of the three U.S. interconnections were studied using dynamic simulation tools. The study demonstrated that dynamic simulation is effective in assessing the frequency-response-based reliability for utility-defined future scenarios. In the simulation, different what-if scenarios can be explored such as different penetration levels of renewable or dispatch strategies.

For example, Figure 25 presents simulation results for the three levels of wind generation output corresponding to $1 \mathrm{GW}, 4 \mathrm{GW}$, and $9 \mathrm{GW}$ for the high-reserves case. Each case shows the evolution of system frequency over the first 19 seconds following the sudden loss of 2,800 MW of generation from two large generating plants in the southwestern portion of the Western Interconnection (Eto et al. 2010).

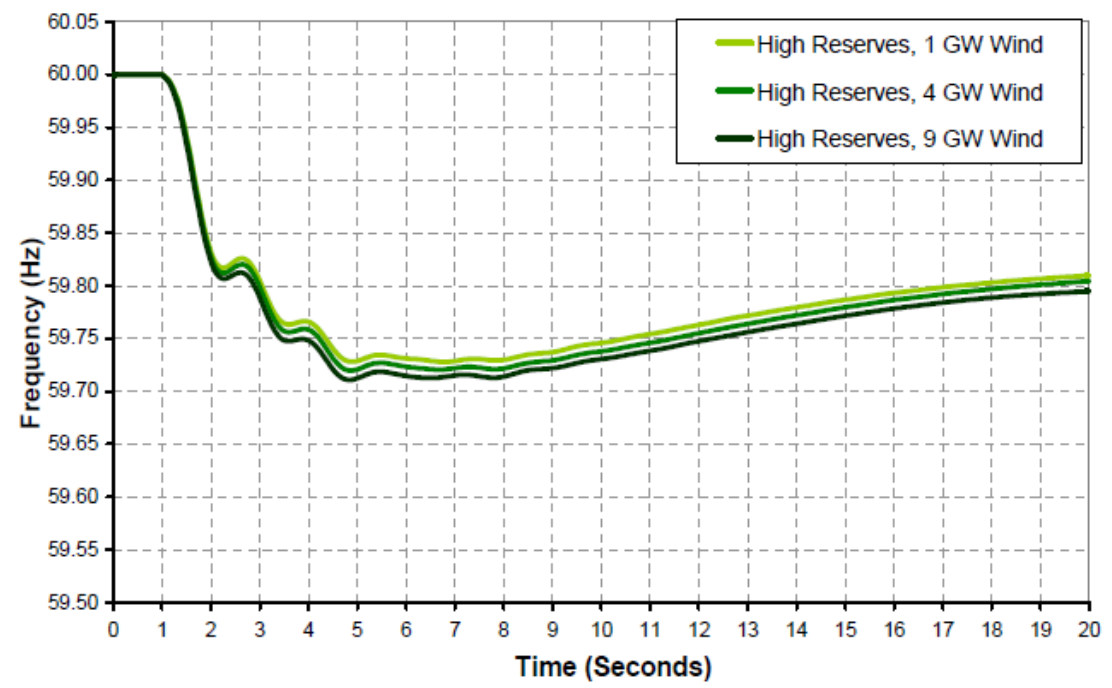

Figure 25. Simulated Western Interconnection system frequency over the first 19 seconds following the sudden loss of the $\mathbf{2 , 8 0 0 ~} \mathrm{mw}$ of generation for the high reserves case (Eto et al. 2010).

To ensure that the simulations are representative of current practices, four issues have been successfully addressed in the study (Eto et al. 2010):

"First, all simulations were conducted using the same commercially available, production-grade simulation tools that are currently used by industry: the General Electric (GE) Positive Sequence Load Flow (PSLF) for the Western Interconnection and the Siemens Power Technologies International Power System Simulator for Engineering (PSS/E) for the Texas and Eastern Interconnections... The tools contain detailed representations of the operation of generators and their automatic controls, the transmission system (including the operation of under-frequency and other load-shedding relays), and the behavior of loads. These tools are routinely used by

${ }^{83}$ Contributed by Pengwei Du, PNNL.

${ }^{84}$ Reused by permission of FERC. 
the industry to study the dynamic performance of the interconnections in response to system events that result in major perturbations of voltage, frequency, and flows of power.

"Second, planners within each interconnection helped to develop the system models to represent conditions expected in 2012.

"Third, the loss of generation event studied is based on an actual event involving the loss of a large amount of generation within each interconnection.

"Fourth, modeling practices had been followed in conducting the simulations. When the cases were prepared for simulation, generation redispatch and operating reserves levels were established separately for each Balancing Authority within the interconnection to maintain power flows among Balancing Authorities at levels established in the interconnections' system model. In conducting the simulations, minor adjustments had been made to ensure that voltage limits defined for the cases were observed" (Eto et al. 2010).

On the other hand, today's dynamic simulation tools and system modeling practices are intended primarily to study system performance over about 20 to 40 seconds of operation following a discrete event. The significance of this limitation for the simulation-based findings presented in this section is that all findings, with respect to the primary frequency control performance, are subject to the adequacy of secondary frequency control reserves (Eto et al. 2010).

For the simulations, a principal finding is that the rapid delivery of power, via primary frequency control actions, is more important than the amount of wind generation in determining the frequency nadir. The effect of increased wind generation in lowering system inertia is not significant compared to the effects of primary frequency control actions. The simulations also suggest that focused attention on the quality of primary frequency control actions, provided by generator governors and, in the Texas Interconnection, frequency-responsive demand response, can readily off-set the effects of increased wind generation on system inertia (Eto et al. 2010).

\subsubsection{Existing and proposed frequency response standards}

The WECC MORC (minimum operating reliability criteria) requires that each Balancing Authority carry contingency reserve sufficient to cover at least the greater of:

1. The most severe single contingency; or

2. Five percent of the load responsibility served by hydro generation, plus 7 percent of the load responsibilities served by thermal generation.

At least half of this reserve must be spinning reserve. Furthermore, spinning reserve must be synchronized and automatically responsive to reduction in frequency.

The total frequency reserve magnitude is based on the size of the disturbance that WECC intends to withstand without going into the first step of under-frequency load shedding $(59.5 \mathrm{~Hz})$. However, as demonstrated in Eto et al. (2010), the rapid delivery of power via primary frequency control actions is more important than the amount of wind generation in determining the frequency nadir. To ensure that adequate primary, frequency control reserves with the appropriate response characteristics are in place at all times (especially when the penetration of renewable is high), current practice and standards for frequency-response-based reliability should be revisited. Thus, recommendations for future development have been given as follows (Eto et al. 2010): 
- Comprehensive planning and enhanced operating procedures, including training, operating tools, and monitoring systems, should be developed that explicitly consider interactions between primary and secondary frequency control reserves, and address the new source of variability that is introduced by wind generation.

- Requirements for adequate frequency control should be evaluated in assessments of the operating requirements of the U.S. electric power system when considering new potential sources of generation, such as solar and additional nuclear generation and the retirement of existing generation.

\subsection{Western Renewable Energy Zones}

"The publication of "Western Renewable Energy Zones - Phase 1 Report" marks an historic turning point for the West and its energy future" (Western Governors Association and U.S. Department of Energy 2009). The important outcomes of this report are:

1. It takes important steps toward identifying the Western Renewable Energy Zones, those areas throughout the Western Interconnection that feature the potential for large scale development of renewable resources in areas with low environmental impacts, subject to resource-specific permitting processes.

2. This Report marks the completion of important work to assist evaluating various transmission strategies. The intention of the WREZ initiative is not simply to identify renewable energy zones in the Western Interconnection, but to facilitate the development of high-voltage transmission to those areas with the potential for abundant renewable resources, and low or easily mitigated environmental impacts.

3. This Report identifies the breadth of renewable energy potential across the Western Interconnection, beyond the potential that will be identified in the Western Renewable Energy Zones (WREZ)" (Western Governors Association and U.S. Department of Energy 2009).

\subsubsection{The Path Toward Western Renewable Energy Zones}

"In Phase 1, the Western Renewable Energy Zones (WREZ) stakeholders engaged in the fundamental challenge and opportunity of the initiative: identifying WREZ that satisfy a diverse range of criteria to support large-scale transmission investment. To develop those criteria, the working groups established a process that evaluated promising resource areas through several steps. Figure 26 shows the steps being taken by the initiative to move from identification of renewable resources to a WREZ. Presently, the initiative has identified Qualified Resource Areas, but not Western Renewable Energy Zones. The final identification will depend on the evaluation and public comment process relating to wildlife information and additional information from load-serving entities" (Western Governors Association and U.S. Department of Energy 2009). 


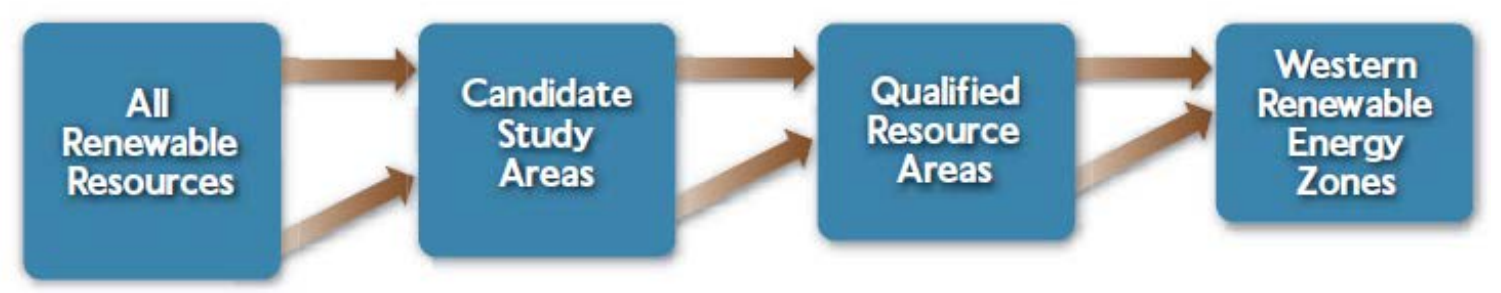

Figure 26. Steps being taken to move from identification of renewable resources to a WREZ (Western Governors Association and U.S. Department of Energy 2009). ${ }^{85}$

\subsubsection{Renewable Resources}

"The initial filter in evaluating the renewable energy resources was to identify those resources that met a threshold potential for commercial development. The resource review and application of the respective thresholds is outlined below. The Zone Identification and Technical Analysis (ZITA) work group analyzed wind, solar, geothermal, biomass and hydropower resource potential by examining raw data and maps from the U.S. Department of Energy's National Renewable Energy Lab and Idaho National Research Lab, the Western Governors' Association Western Bioenergy Assessment, as well as Canadian renewable resource data obtained from a variety of sources. ZITA divided the Western Interconnection's renewable resources into two categories:

- Primary - Large amounts of renewable energy potential significant enough to define a Western Renewable Energy Zone's boundaries. These are the resources with the greatest near-term generation potential across the Western Interconnection.

- Secondary - Amounts of renewable energy potential small enough that the resource in itself would not define a Western Renewable Energy Zone's boundaries, but could be included in a WREZ once quantified" (Western Governors Association and U.S. Department of Energy 2009).

\subsubsection{Forming the Candidate Study Areas}

"The original NREL resource maps identified vast amounts of commercially viable renewable energy potential in the Western Interconnection, including more than 2 million megawatts of potential wind power resources and several million megawatts of potential solar energy resources. As a frame of reference, the peak load for the entire WECC in 2007 was approximately 150,000 megawatts. In order to reduce the large potential to only the best resources, some additional filtering was applied" (Western Governors Association and U.S. Department of Energy 2009).

\subsubsection{Refining to Qualified Resource Areas}

"The ZITA working group determined that the minimum size of a Qualified Resource Area should be based on the electrical generating potential sufficient to justify at least a $500 \mathrm{kV}$ alternating current transmission line: $1,500 \mathrm{MW}$ for variable resources with moderate capacity factors, such as wind and solar. ${ }^{86}$ ZITA also established a maximum size of approximately 100

\footnotetext{
${ }^{85}$ Reused by permission of Western Governor's Association.

86 "Geothermal and biomass resources have, on average, two to three times the capacity factor of wind and solar. To account for this when identifying Qualified Resource Areas (QRAs), the capacity of these
} 
miles from the geographic center of a Qualified Resource Area. ZITA concluded that a distance ${ }_{87}$ reater than this would unreasonably increase the costs of connecting to the transmission grid.

The results of this analysis were geographic areas with at least 1,500 MW of high-quality renewable energy within a 100 mile radius" (Western Governors Association and U.S. Department of Energy 2009).

\subsubsection{WREZ Initiative Hub Map}

"Hubs shown on the map (Figure 27) are labeled by the abbreviation for each state and province, as well as by the geographical area, such as NE for northeast. 'Hubs' are graphical representations of regional renewable resource potential in the Western Interconnection, identified for purposes of evaluating interstate transmission lines in future phases of the initiative" (Western Governors Association and U.S. Department of Energy 2009).

resources in a QRA counts triple when considering whether a QRA meets the 1,500 MW threshold. The actual amount of geothermal and biomass resource is quantified in the energy and capacity calculations. Due to the greater relative certainty of the developability of resources identified in Alberta and British Columbia, this minimum threshold was relaxed for wind resources in these provinces” (Western Governors Association and U.S. Department of Energy 2009).

${ }^{87}$ Qualified Resource Areas, Selection Methodology, February 2009, ZITA, Western Governors’ Association (WGA). 


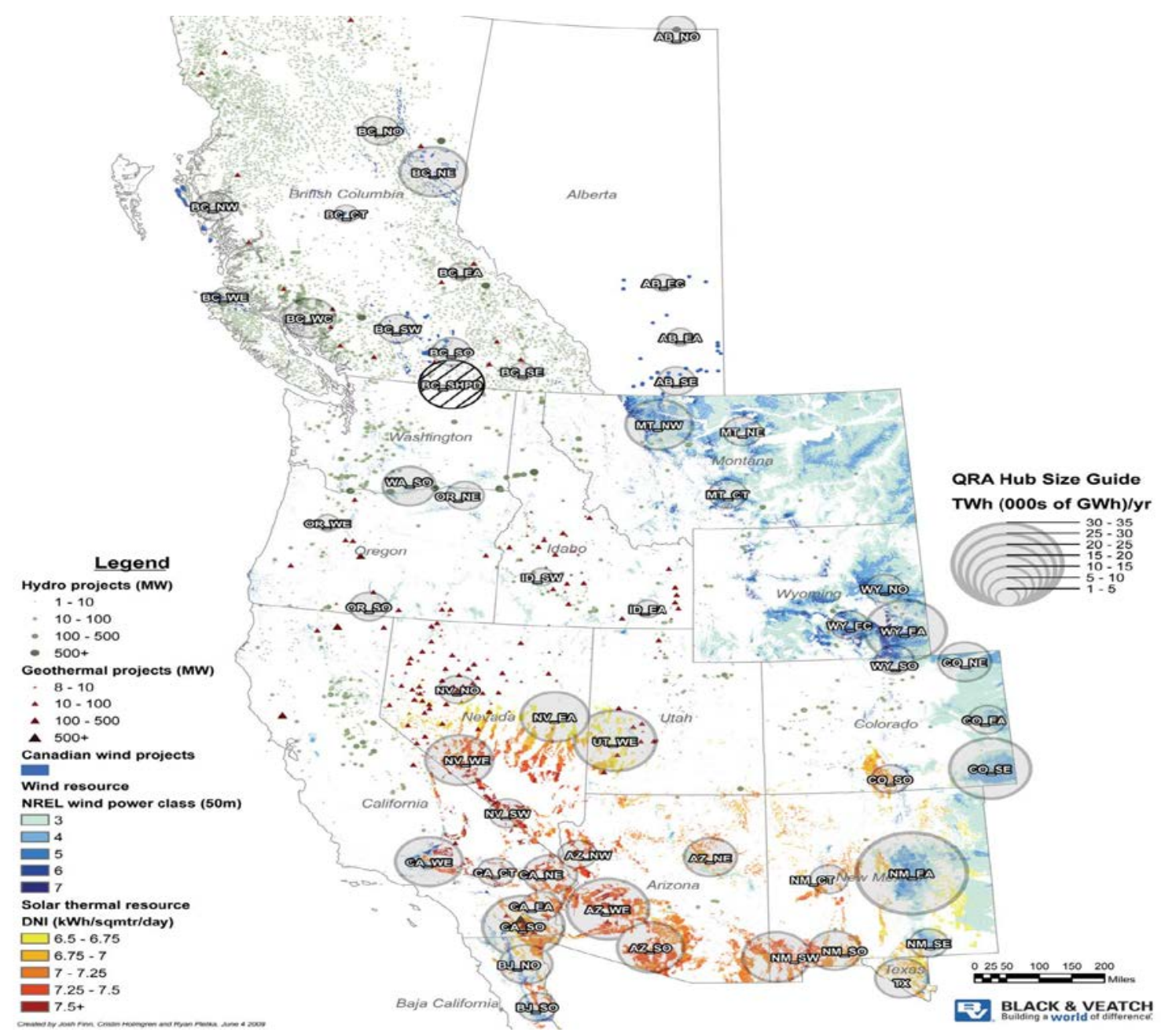

Figure 27. WREZ Initiative Hub Map. ${ }^{88}$

WREZ initiative renewable energy generating capacity tables are presented in Appendix 23.

${ }^{88}$ Reused by permission of Western Governor's Association. 


\section{Extreme Events, Cascading Failures, and Blackouts}

"Power system cascading failures may occur due to the loss of several important elements, such as multiple generating units within a power plant, parallel transmission lines or transformers and common right-of-way circuit outages. The failure of these elements may widely propagate through the interconnected power network and result in a local or wide-area blackout. These kinds of failures that cause severe consequences are initiating events to a cascading failure" (Morgan et al. 2011).

"Substantial cascading events are rare because often the initial system state is robust enough that it withstands the first few events and the cascade stops. But in an unfavorable initial system state, a trigger event can lead to many further events that become a substantial cascade and blackout. It is these large cascading events that cause the most damage and are classified as "extreme events" (Morgan et al. 2011).

"A blackout in an electric system means that the complete system collapses. Such a blackout affects all electricity consumers in the area. It can originate from several causes. One example is the loss of generation, e.g., the trip of a power plant that causes a mismatch between production and load. This puts a strain on other generators, resulting in under-frequency in the system while it 'catches up,' and may result in the further loss of other generators. Another example is an overload of the transmission system caused by congestion, forcing an overloaded power line to trip, causing increased loading of other lines those results in additional trips, and in the end - a voltage collapse due to the high impedance in the weakened grid. In general, one initial event. which might be considered to be minor, leads to a second event, a third and so forth, with increased stresses on the system which finally collapses. The course of events is an example of the well-known 'Domino Effect' (ABB 2003). Some of the blackout events are listed in Appendix 13. 


\section{Considerations for Energy Storage}

This section considers various energy storage technologies and their application.

\subsection{Energy storage technologies and applications ${ }^{89}$}

Energy storage design and sizing is a function of the application and the desired life. Energy storage for applications that are of short duration are tailored for high power to energy ration, while the reverse is true for applications of long duration. "Size and weight of storage devices are important factors for certain applications. Figure 28 shows output energy densities (product of input energy density and efficiency) of different energy storage technologies. The energy density ranges reflect the differences among manufacturers, product models and impact of packaging"( Electricity Storage Association).

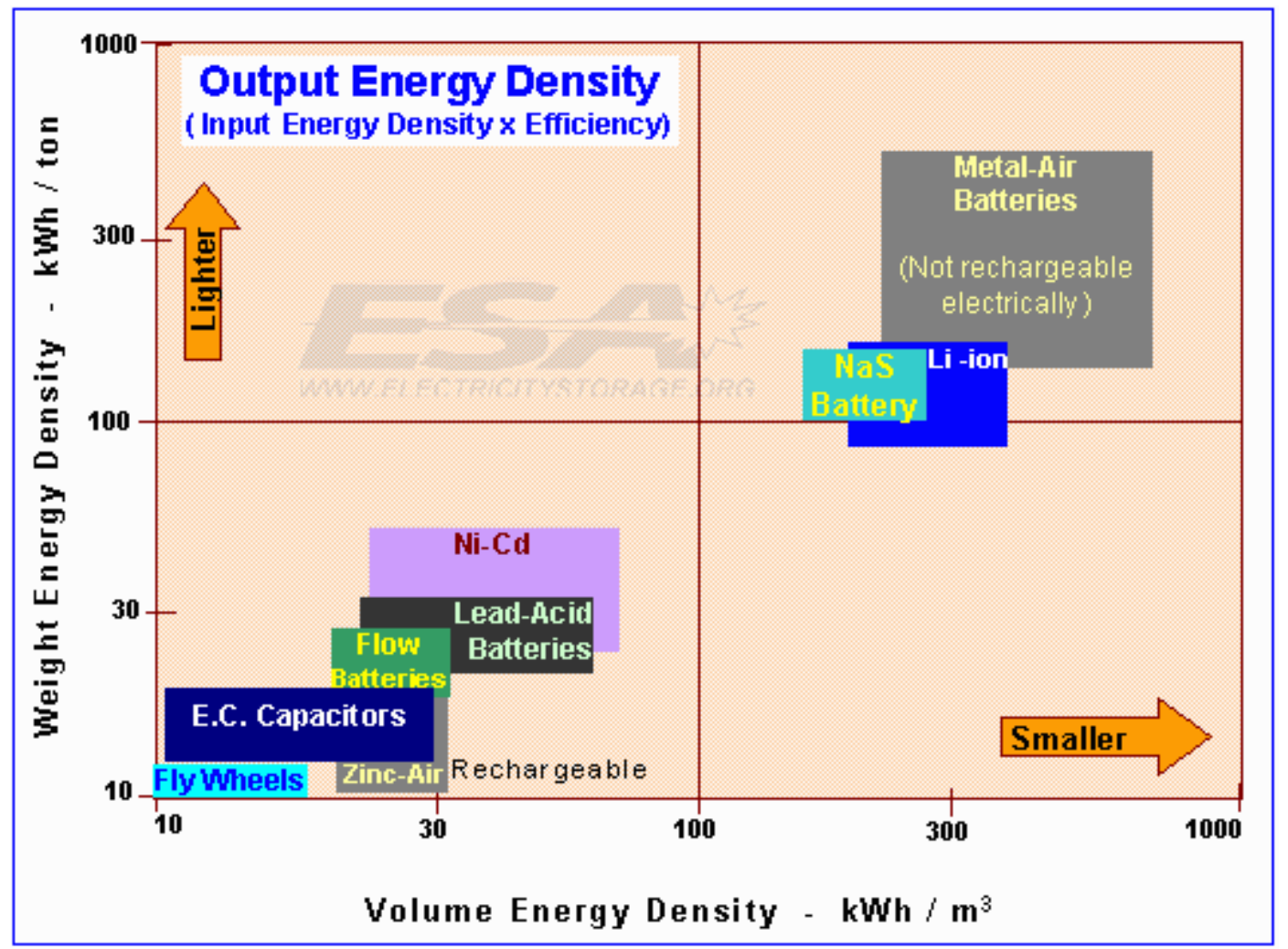

Figure 28. Output energy densities of different energy storage technologies. ${ }^{90} 91$

"Scale stationary applications of electric energy storage can be divided in three major functional categories:

1. Power Quality - Stored energy, in these applications, is only applied for seconds or less, as needed, to assure continuity of quality power.

\footnotetext{
${ }^{89}$ Contributed by Vilayanur Viswanathan, PNNL.

${ }^{90}$ http://www.electricitystorage.org/technology/storage_technologies/technology_comparison

${ }^{91}$ Reused by permission of the Electricity Storage Association.
} 
2. Bridging Power - Stored energy, in these applications, is used for seconds to minutes to assure continuity of service when switching from one source of energy generation to another.

3. Energy Management - Storage media, in these applications, is used to decouple the timing of generation and consumption of electric energy. A typical application is load leveling, which involves the charging of storage when energy cost is low and utilization as needed. This would also enable consumers to be grid-independent for many hours.

Although some storage technologies can function in all application ranges, most options would not be economical to be applied in all three functional categories. Figure 29 shows system ratings of different energy storage technologies" (Electricity Storage Association). ${ }^{92}$

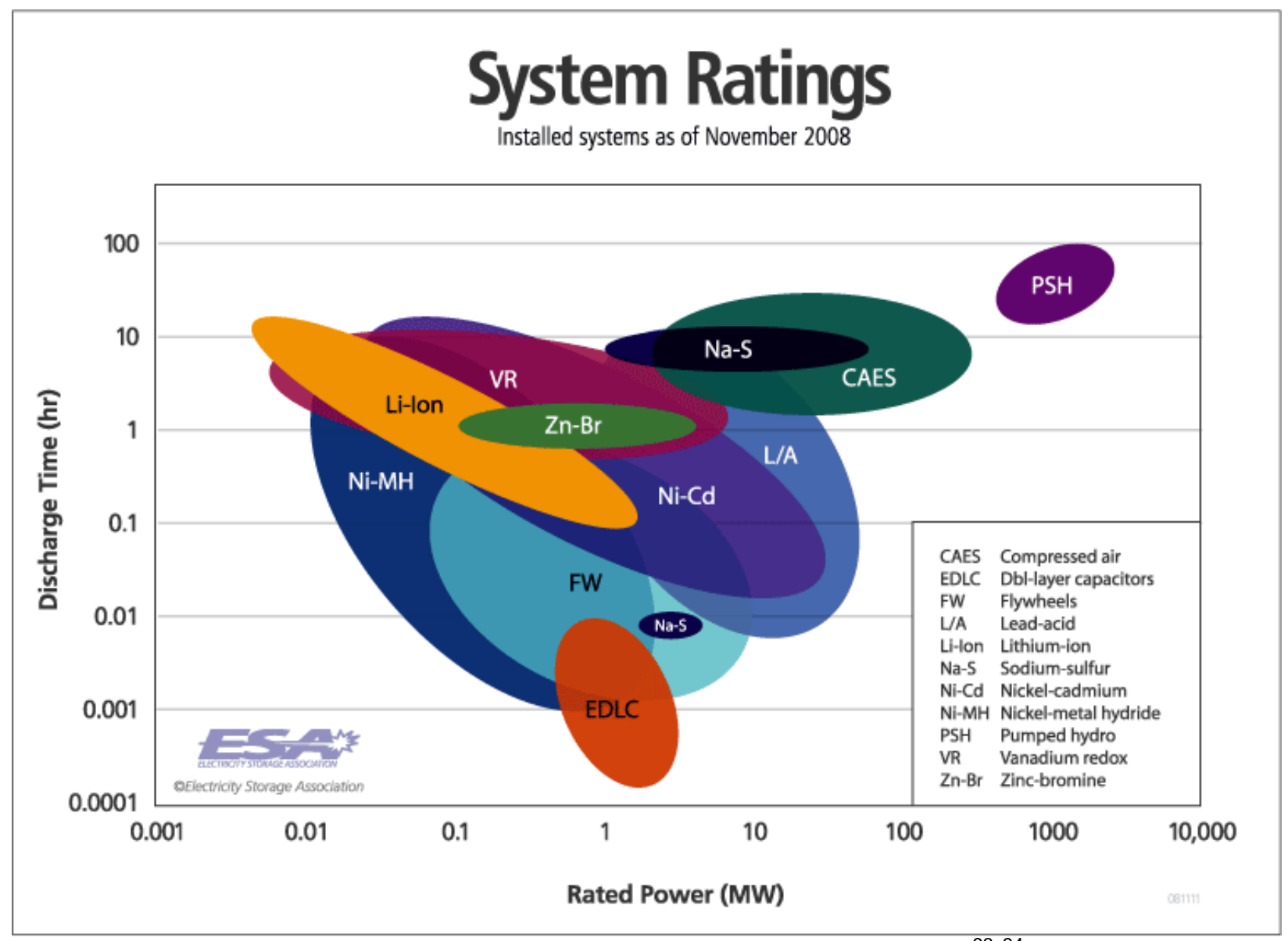

Figure 29. Ratings of various energy storage technologies. ${ }^{93} 94$

This section addresses applications for energy storage and associated benefits:

92 http://www.electricitystorage.org/technology/storage_technologies/technology_comparison

93 http://www.electricitystorage.org/technology/storage_technologies/technology_comparison

${ }^{94}$ Reused by permission of the Electricity Storage Association. 


\subsubsection{Energy storage applications and benefits}

As wind and solar generation gain market share, there is the need to firm up the wind both over the short and long term. Below are some of the anticipated applications for which energy storage can be used:

Energy storage can be used for a variety of applications, with several reports on its benefits (Eyer and Brown 2007). The 14 possible energy storage applications (grouped by category) are:

\section{Grid Capacity and Energy}

1. Electric energy arbitrage

2. Electric capacity (power)

3. Reduce needed transmission capacity

4. Reduce transmission congestion

5. Transmission and distribution upgrade deferral

\section{Ancillary Services}

6. Operating reserves

7. Regulation

8. Transmission support

\section{End-user/Third Party}

9. Electric service reliability

10. Electric service power quality

11. Electric service bill reduction: demand charges

12. Electric service bill reduction: time-of-use energy pricing

\section{Renewables}

13. Renewables energy production time shift

14. Renewables capacity (power) firming.

Table 7 provides more information on energy storage applications and benefits. The maximum market potential is the maximum amount of storage capacity that could be used for the application. The unit benefit is the net present value of estimated benefits of energy storage over a 10-year period using an appropriate inflation and discount rate, normally at 2.5 percent and 10 percent, respectively. 
Table 7. Storage applications and benefits (Eyer and Brown 2007) ${ }^{95}$

\begin{tabular}{|c|c|c|c|c|}
\hline \# & Application & Benefit & Description & $\begin{array}{l}\text { Cost Element(s) } \\
\text { or Price Signal(s) }\end{array}$ \\
\hline 1 & $\begin{array}{l}\text { Electric Energy } \\
\text { Buy Low - Sell High }\end{array}$ & $\begin{array}{l}\text { Revenue - VOC - } \\
\text { (Purchase - Efficiency) }\end{array}$ & $\begin{array}{l}\text { 1. Avoided market-based cost for } \\
\text { purchases or } 2 \text {. "Profit" from selling. }\end{array}$ & LBMP DAM \\
\hline 2 & Electric Supply Capacity & Installed Capacity (ICAP) & $\begin{array}{l}\text { Avoid charges/receive payment for } \\
\text { "supply" installed capacity (ICAP). }\end{array}$ & $\begin{array}{l}\text { NYISO ICAP } \\
\text { Strip Auction }\end{array}$ \\
\hline 3 & $\begin{array}{l}\text { Reduce Transmission Capacity } \\
\text { Requirements }\end{array}$ & $\begin{array}{l}\text { Reduced Transmission } \\
\text { Service Charges (TSCs) }\end{array}$ & $\begin{array}{l}\text { Avoid payment of charges incurred for } \\
\text { access to the transmission system. }\end{array}$ & $\begin{array}{l}\text { NYISO Transmission } \\
\text { Service Charge (TSCs) }\end{array}$ \\
\hline 4 & $\begin{array}{l}\text { Reduce Transmission } \\
\text { Congestion }\end{array}$ & $\begin{array}{l}\text { Reduced Transmission } \\
\text { Congestion Costs }\end{array}$ & $\begin{array}{l}\text { Reduce congestion on transmission } \\
\text { system(s) - to reduce congestion- } \\
\text { related cost - by serving peak load } \\
\text { with storage. }\end{array}$ & $\begin{array}{l}\text { LBMP DAM (Congestion } \\
\text { Component) }\end{array}$ \\
\hline 5 & $\begin{array}{l}\text { Transmission and Distribution } \\
\text { Upgrade Deferral }\end{array}$ & $\begin{array}{l}\text { Avoided Annual Revenue } \\
\text { Requirement for T\&D } \\
\text { Upgrade }\end{array}$ & $\begin{array}{l}\text { Defer need for relatively expensive } \\
\text { T\&D upgrades by serving peak load } \\
\text { downstream from hot spots. }\end{array}$ & $\begin{array}{l}\text { Annual revenue } \\
\text { requirement for upgrade. }\end{array}$ \\
\hline $\mid 6$ & Operating Reserve & Operating Reserve, Value & $\begin{array}{l}\text { "Back-up" for Emergencies (loss of one } \\
\text { or two large resources) }\end{array}$ & $\begin{array}{l}\text { DAM Prices (LBMP and } \\
\text { reserve capacity) }\end{array}$ \\
\hline 7 & $\begin{array}{l}\text { Regulation and Frequency } \\
\text { Response (Regulation) }\end{array}$ & Regulation Service, Value & $\begin{array}{l}\text { Maintain grid stability, frequency; } \\
\text { attenuate small, frequent load } \\
\text { fluctuations. }\end{array}$ & DAM Prices \\
\hline 8 & Transmission Support & $\begin{array}{l}\text { Enhanced Transmission } \\
\text { Performance }\end{array}$ & $\begin{array}{l}\text { Short duration support for transmission } \\
\text { stability and improved throughput. }\end{array}$ & n/a \\
\hline 9 & Electric Service Reliability & $\begin{array}{l}\text { Reduced Outage Related } \\
\text { Cost }\end{array}$ & $\begin{array}{l}\text { Financial losses avoided due to } \\
\text { improved } P Q \text {. }\end{array}$ & Value-of-Service as proxy \\
\hline 10 & Electric Service PQ & Reduced PQ-related Cost & $\begin{array}{l}\text { Financial losses avoided due to } \\
\text { improved PQ. }\end{array}$ & Value-of-Service as proxy \\
\hline 11 & $\begin{array}{l}\text { Electric Service Bill Reduction: } \\
\text { Demand Charges }\end{array}$ & $\begin{array}{l}\text { Reduced Electric Service } \\
\text { Bill }^{2}\end{array}$ & Reduced electricity bill. & $\begin{array}{l}\text { Tariff. PSC No. 9, Service } \\
\text { Class 9, Rate I }\end{array}$ \\
\hline 12 & $\begin{array}{l}\text { Electric Service Bill Reduction: } \\
\text { Time-of-use Energy Prices }\end{array}$ & $\begin{array}{l}\text { Reduced Electric Service } \\
\text { Bill }^{2}\end{array}$ & Reduced electricity bill. & $\begin{array}{l}\text { Tariff: PSC No. 9, Service } \\
\text { Class 9, Rates II \& III + } \\
\text { Market Supply Charges }\end{array}$ \\
\hline 13 & $\begin{array}{l}\text { Renewable Electricity } \\
\text { Production Time-shift }\end{array}$ & $\begin{array}{l}\text { Enhanced Wind Energy } \\
\text { Value }\end{array}$ & $\begin{array}{l}\text { Increased benefit from wind energy if } \\
\text { low value wind energy is sold when } \\
\text { value is high. }\end{array}$ & $\begin{array}{l}\text { DAM LBMP and "firmed } \\
\text { capacity" (ICAP) Credit. }\end{array}$ \\
\hline $\mid 14$ & Renewables Capacity Firming & $\begin{array}{l}\text { Enhanced Photovoltaics } \\
\text { Capacity Value }\end{array}$ & $\begin{array}{l}\text { Increase benefit from PV using low } \\
\text { value grid energy to firm-up PV } \\
\text { capacity on peak. Firming: from } .5 \\
\text { to. } 95 \text { effective capacity (Summer). }\end{array}$ & $\begin{array}{l}\text { DAM LBMP and "firmed } \\
\text { capacity" (ICAP) Credit. }\end{array}$ \\
\hline
\end{tabular}

Table 8 provides more information on energy storage applications and applicable benefits.

${ }^{95}$ Reused by permission of New York State Energy Research and Development Association (NYSERDA) 
Table 8. The Application matric- A Logical Analytical Framework (Eyer, J. 2012). . ${ }^{96}$

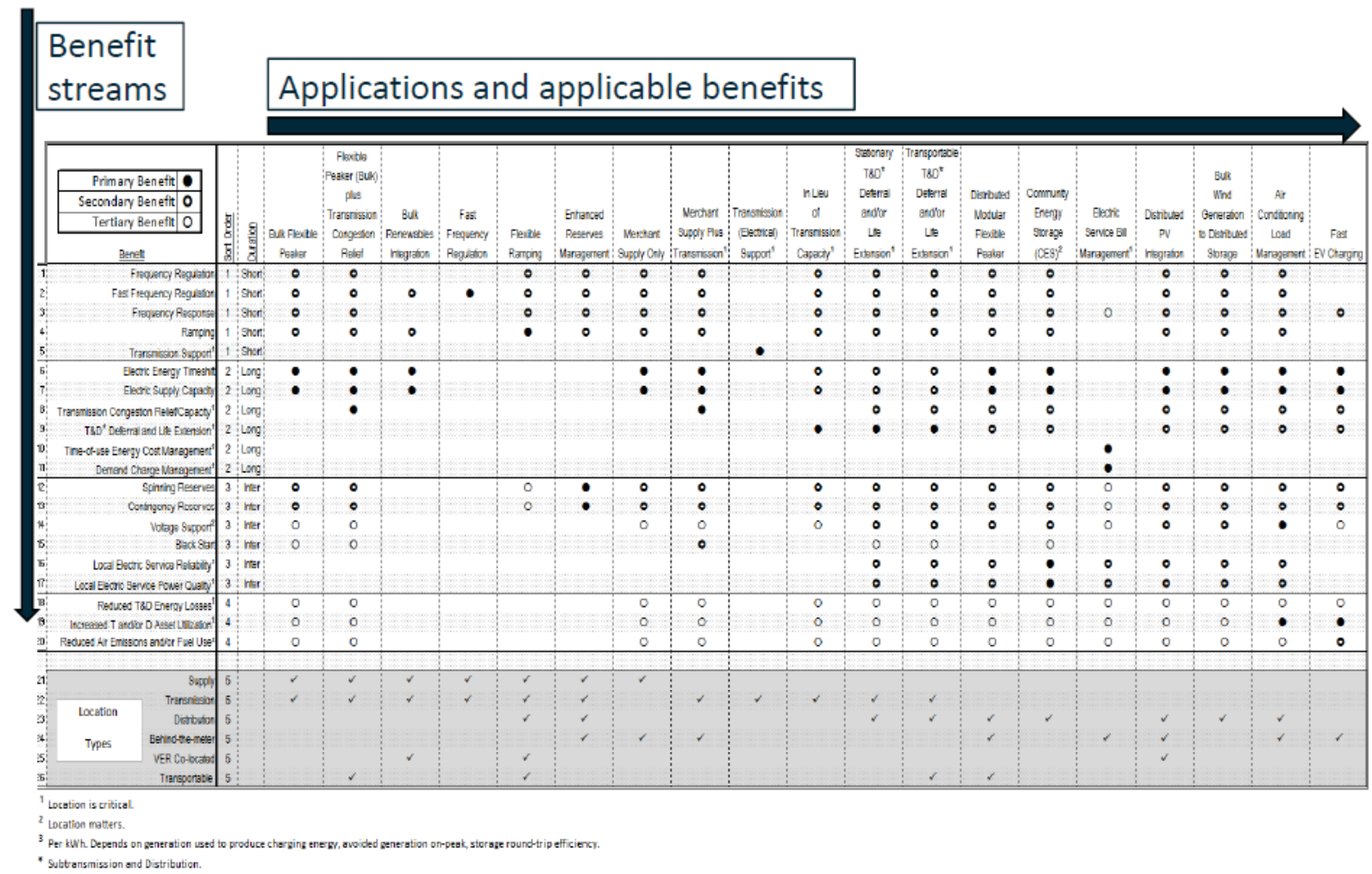

\subsubsection{Energy storage technologies}

Energy storage options come in various degrees of technological maturity. What follows are some of the energy storage technologies' features:

\subsubsection{Lead-acid batteries}

"As a well-developed. advanced battery technology, lead-acid battery systems are easily sited and have been employed in multi-megawatt level assemblies for load leveling and grid stabilization. Compared with other advanced battery technologies, they have shallow depth of discharge, lower energy density and higher capital cost, which limit their application on regulation services" (Makarov et al. 2008c). Lead-acid batteries have an expected cycle life of several thousand cycles at the 2 percent to 5 percent depth of discharge (DOD) corresponding to each charge.

"It is an electrochemical battery, based on lead electrodes submerged in sulfuric acid. When the battery is discharged, lead $(\mathrm{Pb})$ is converted to lead sulfate $\left(\mathrm{PbSO}_{4}\right)$ at the anode (releasing two electrons), and lead oxide $\left(\mathrm{PbO}_{2}\right)$ to lead sulfate $\left(\mathrm{PbSO}_{4}\right)$ at the cathode (absorbing two electrons). The process is theoretically reversible, but a layer of non-convertible lead sulfate tends to build up at the electrodes during cycling. This problem is particularly significant if the battery is left in a discharged state for long periods. Other problems with this technology include corrosion of the electrodes and the shedding of active material from the electrode as a result of volume change of the material during deep discharge. The technology is relatively inexpensive

\footnotetext{
${ }^{96}$ Reused by permission of Strategen
} 
and easily sited, and has been employed in multi-megawatt assembles for load leveling and grid stabilization."

While lead-acid batteries are universally used for starting, lighting and ignition, these batteries with thin electrodes have long life because of the shallow cycling they are subjected to, with an expected cycle life of several thousand cycles at the 2 percent to 5 percent DOD corresponding to each cycle. Electrodes modified with calcium are more resistant to corrosion, overcharging, water loss and self-discharge. They need little to no maintenance and provide higher cold cranking current. Deep cycle batteries consist of thick electrodes to increase the specific energy and energy density, and to maximize life. Electrodes modified with antimony improve electrode mechanical strength, reduce water loss due to gassing, have longer service life than calciumincorporated electrodes, are faster to recharge from a fully discharged state and cost less. These batteries are preferred for deep discharge application.

Lead-acid batteries are of flooded electrolyte or starved electrolyte design. In the former, excess electrolyte is used, and periodic topping off with water may be necessary. The starved electrolyte design uses just enough electrolyte to provide an ionic path between the electrodes. The electrolyte is trapped either in an absorbent glass separator or in a gel, with sufficient voids present in the separator for gas flow between electrodes. The amount of electrolyte added is controlled such that a very thin film is present at the electrodes for rapid gas recombination.

"The largest demonstration for electric utility applications in the United States is a 20-MW/18MWh plant in San Juan, Puerto Rico, providing spinning reserve, frequency control, and voltage control. (Makarov et al. 2008c). A successful example of a valve-regulated, lead-acid battery system installation was that of a 1-MW/1.4-MWh system installed by Exide Technologies at the island village of Metlakatla, Alaska, to stabilize the island's power grid. ${ }^{97}$ Post-mortem of the cells after 11.5 years of continuous operation showed that the electrodes were quite healthy, thus showing promise for this old technology to be a serious player in this field.

\subsubsection{Electrochemical capacitors}

"Superior to most conventional battery systems in speed of response and cycle life, super capacitors are a developing technology that is not yet applicable for the storage requirements of electric utility-scale operations. Like traditional dielectric capacitors, the super capacitor (or ultracapacitor) stores energy by physically separating negative and positive charges. The energy density of super capacitors is, however, much higher than that of traditional capacitors as a result of a modified internal capacitor structure. The short charge and discharge time (fractions of seconds) known from traditional capacitors is partially maintained (few seconds), as well as the very long cycle life (potentially 100,000s of cycles). Accordingly, the super capacitor is said to gap the bridge between batteries and capacitors in terms of energy density and response time. Super capacitors are a rapidly developing technology and a strong research effort in continuously improving the energy density.

Current applications typically take place in combination with batteries or other storage or power supplies, in situations where a low average, but high pulse, power is needed. An example is cars, where the use of super capacitors can decrease the needed battery size or in buses, where the capacitors store braking energy and release it during acceleration.

\footnotetext{
97 http://www.restoreenergysystems.com/images/ideas-in-action/MetlakatlaPaperJan292010.pdf accessed June 26,
} 2012. 
The technology is not yet considered applicable for the storage requirements of power system regulation or other large-scale electric storage operations" (Makarov et al 2008c).

A cost comparison of various types of capacitors is given below (Burke 2007). The cost for electrochemical double layer capacitors (EDLC) is highly sensitive to carbon price. At a unit price of $\$ 10 / \mathrm{kg}$, it is significantly more expensive than $\mathrm{PbO}_{2} / \mathrm{C}$, whose cost was estimated by using a higher price of $\$ 4.5 / \mathrm{kg}$ compared to the $\$ 2-4 / \mathrm{kg}$ for lead-acid batteries. For unit price of $\$ 10 / \mathrm{kg}$, the device price can be kept at 0.2 cents per Farad for $2.7 \mathrm{~V} /$ cell, which is lower than the 0.5 cents per Farad mentioned as reasonable for carbon/carbon devices using acetonitrile as electrolyte in this article. It should be noted that the loxus capacitors were quoted at 5 cents/Farad for high volume $1000^{\circ} \mathrm{F}$ capacitor, and 2.3 cents/Farad for high volume 3000 Farad capacitor $^{98}$ (Buckley 2010). The Maxwell $56 \mathrm{~V} / 130^{\circ} \mathrm{F}$ modules cost $\$ 1,000$ for large quantities, corresponding to 1.8 cents per Farad, and a cost of $\$ 100 / \mathrm{kW}$ and $\$ 23,500 / \mathrm{kWh}$. The costs for single-cell 3,000 $\mathrm{F}$ loxus capacitors are $\$ 90$ and $\$ 70$ for low and high volume, respectively, corresponding to $\$ 30,000 / \mathrm{kWh}$ and $\$ 23,000 / \mathrm{kWh}$, respectively.

\subsubsection{3 $\mathrm{C}-\mathrm{PbO}_{2}$ hybrid capacitors}

In the lead carbon ( $\mathrm{PbC}$ ) battery, the lead negative electrode is replaced by a super capacitor electrode made of activated carbon, with a surface area as high as $1,500 \mathrm{~m}^{2} / \mathrm{g}$. The positive electrode undergoes the same reaction, while the negative electrode stores $\mathrm{H}^{+}$ions at the electrode surface, and exchanges it with the electrolyte during charge and discharge. The $\mathrm{H}+$ ions are stored in the negative electrode during the charged state, and transported to the positive electrode during discharge for neutralization to water. This leads to lower acid concentration swings from the charge to discharge state, reducing grid corrosion on the positive electrode and extending its life.

\footnotetext{
${ }^{98}$ http://www.ioxus.com
} 


\section{Ultrabattery}

A new type of energy storage device is a battery-capacitor hybrid device developed by Commonwealth Scientific and Industrial Research Organization (CSIRO) and licensed to East Penn and Furukawa (Lam et al. 2007, Furukawa et al. 2009). In a conventional lead-acid battery, the failure mechanism was attributed to the build-up of $\mathrm{PbSO}_{4}$ crystals over several cycles, because of lower accessibility of $\mathrm{HSO}_{4}{ }^{-}$ions to the electrode interior. At high-charge rates, the portions of the electrodes that are fully charged can also evolve gas, with associated loss of water. The ultrabattery circumvents this issue by connecting a carbon super capacitor electrode in parallel with the negative spongy lead electrode as shown in Figure 30. The carbon electrode buffers the negative electrode, thus preventing local $\mathrm{PbSO}_{4}$ build-up at high discharge rates. For the hybrid electric vehicle (HEV) application, the ultrabatteries could be cycled from 21-70 percent depth of discharge (DOD) as opposed to 30-70 percent DOD for conventional lead-acid batteries, with a cycle life of 80,000 cycles, an order of magnitude higher than that for conventional lead-acid batteries. See Figure 31.

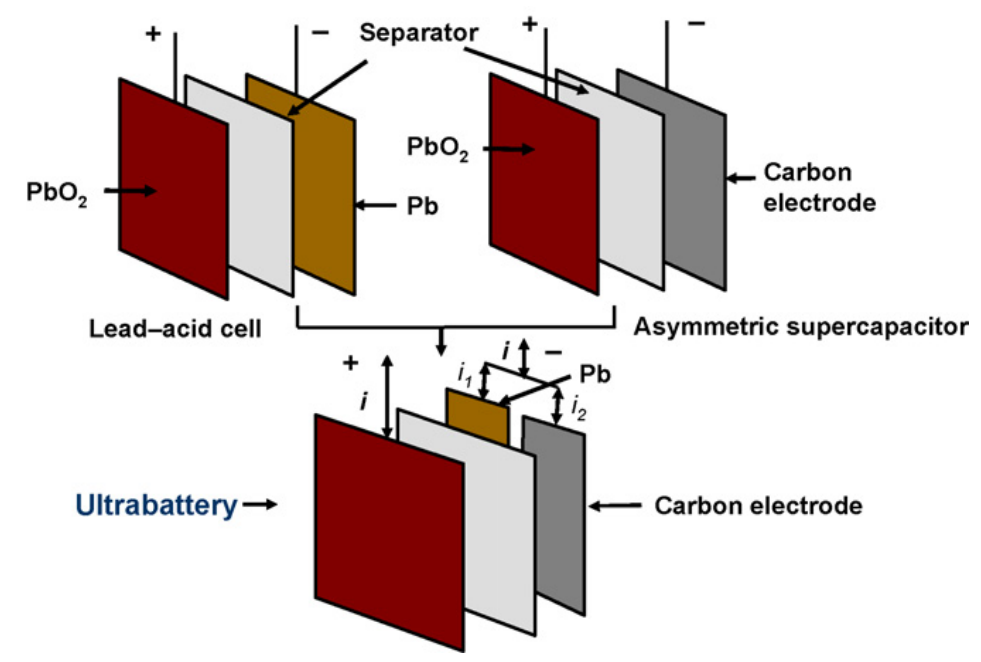

Figure 30. Schematic of the ultrabattery electrode configuration (Lam et al. 2007). ${ }^{99}$

\footnotetext{
${ }^{99}$ Reused with permission of the Electrochemical Society.
} 


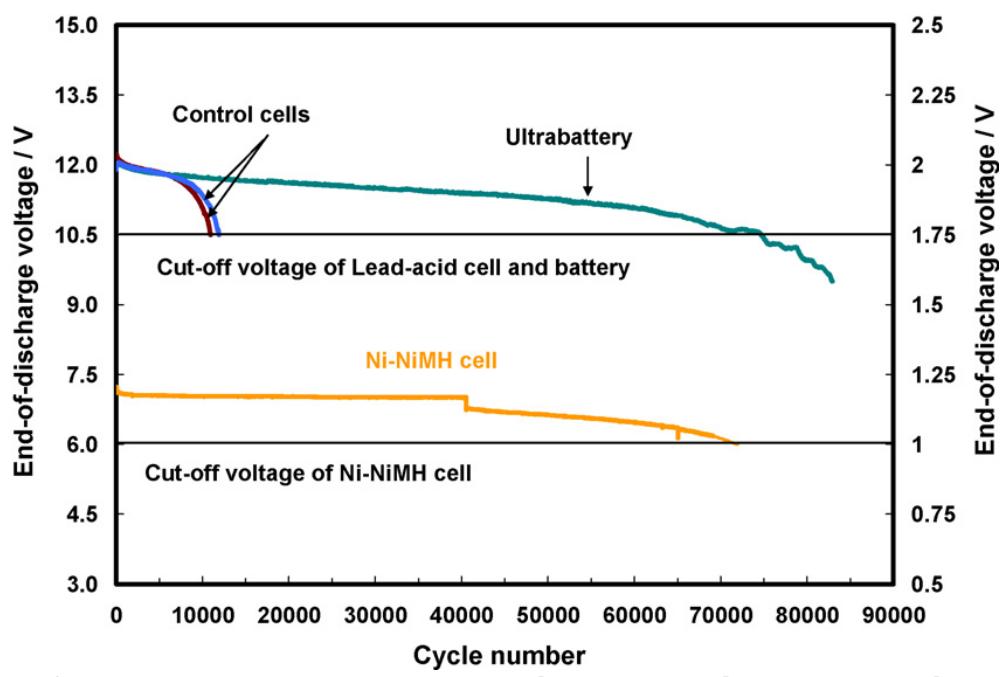

Figure 31. Cycle life of the ultrabattery compared with conventional lead-acid and Ni-MH HEV batteries charge (62s) and discharge $(60 \mathrm{~s})$ at $3^{\circ} \mathrm{C}$ rate, with $10 \mathrm{~s}$ rest after charge and discharge (Lam et al. 2007). ${ }^{100}$

These can be used for improving the reliability and stability of renewable energy, along with load leveling and remote area power supply. The charge and discharge power for renewable energy storage is estimated to be 50 percent higher than that of conventional lead-acid battery. It has been tested and shown to offer advantages over existing Ni-MH batteries used in HEVs in terms of faster-charge, discharge rates and lower cost by 70 percent. A field demonstration for the wind energy storage was conducted by Furukawa Battery Company. ${ }^{101}$

East Penn Manufacturing Co. was awarded a \$2.2M grant from the U.S. DOE (American recovery and reinvestment act (ARRA) for a grid-scale energy storage demonstration using the ultrabattery technology. The system was designed to sell up to $3 \mathrm{MW}$ of frequency regulation to Integrys, a load serving entity of Pennsylvania-New Jersey-Maryland interconnection (PJM) interconnection. It provides demand management service to Metropolitan Edison Electric Utility during specified, peak-power periods for one to four hours, up to $1 \mathrm{MWh}$ (East Penn Manufacturing Co., Inc., 2010).

\section{Axion Power PowerCube ${ }^{\mathrm{TM}} \mathrm{C}-\mathrm{PbO}_{2}$ hybrid capacitors}

Axion Power also has developed $\mathrm{PbC}$ hybrid systems PowerCube ${ }^{\mathrm{TM}}$ for utility-scale energy storage, targeting short-term storage for wind and solar power, with a carbon electrode replacing the negative electrode. These are mobile and can deliver up to $1 \mathrm{MW}$ for 30 minutes, or $100 \mathrm{~kW}$ for 10 hours. Over 2,000 charge-discharge cycles at 100 percent DOD have been demonstrated. ${ }^{102}$

The PowerCube battery, in partnership with Viridity Energy, is being integrated as a 100-kW power resource for the PJM regulation market (Axion Power International 2011). Their technology can be scaled in building blocks of $1 \mathrm{MW}$ of power for 30 minutes for PJM

\footnotetext{
${ }^{100}$ Reused with permission of the Electrochemical Society.

${ }^{101}$ http://www.furukawadenchi.co.jp/english/research/new/pdf/ultra_03.pdf accessed December 4, 2012.

${ }^{102} \mathrm{http}: / /$ www.axionpower.com/profiles/investor/fullpage.asp?f=1\&BzID=1933\&to=cp\&Nav=0\&LangID=1\&s=0\&I D=10298 Accessed January 11, 2012.
} 
applications. It can also provide power quality, back-up power, power smoothing and load leveling, as well as wind and solar energy storage.

For grid utility applications, their storage system can be used for frequency regulation/power smoothing, load shifting, load sharing, peak shaving and solar and wind energy storage. They can build battery packs from $10 \mathrm{kWh}$ to $20 \mathrm{MWh}$ in energy capacity, operating at power levels of $2 \mathrm{~kW}$ to $5 \mathrm{MW}$. They have in their plant a 250-kWh pack that is integrated into the PJM grid operating at power levels of up to $500 \mathrm{~kW} .{ }^{103,104}$.

Their storage systems also are used in automotive start-stop applications, with the batteries being tested in BMW automobiles. These also are used in standby power in commercial vehicles and industrial applications such as heavy trucks, fork lifts, oil rigs, residential power packs, and cranes. Auxiliary power requiring heavy-duty cycling also is provided for buses and trucks. The hybrid/electric vehicle locomotive market also is being targeted.

\subsubsection{Reversible and irreversible fuel cells}

Fuel cells are similar to non-rechargeable batteries in the sense that they can only provide power. To both absorb and provide power, regenerative fuel cells are used. This can be in the form of a combination of an electrolyzer and fuel cell, or as a unitized regenerative fuel cell that can be used to both electrolyze water and to generate electricity. Unitized regenerative fuel cells (URFCs) are a novel technology that is not yet near commercialization. The roadblock to commercial deployment is lack of reliable and high-performance, bi-functional catalysts that can be efficiently used both in the fuel cell and regenerative mode. The National Aeronautics and Space Administration (NASA) has developed a regenerative fuel cell system, which consist of two separate stacks for power generation and hydrogen regeneration (Bents et al. 2005). Graphical representation of 5-kWe fuel cell and electrolyzer stacks that comprise RFC can be found in (Bents et al. 2005).)

Current cost estimates for this system could end up costing at least $\$ 2,500 / \mathrm{kW}$, assuming $\$ 1,000 / \mathrm{kW}$ for each stack and $\$ 500 / \mathrm{kW}$ for the balance of plant. The efficiency for a polymer electrolyte membrane (PEM) fuel cell is 60 percent. Assuming the same efficiency for the regeneration mode, the round-trip efficiency is as low as 36 percent. Hence, this does not appear to be a very viable option both in terms of cost and in terms of efficiency. Alkaline fuel cells (AFC) also have a similar efficiency, with high sensitivity to $\mathrm{CO}_{2}$. The regenerative mode for AFC has not been developed yet. Phosphoric acid fuel cells (PAFC) systems have a low efficiency (40 percent) and operate at between 150 and $200^{\circ} \mathrm{C}$. The power plants developed by United Technologies have a rated power of $200 \mathrm{~kW}$, with over $75 \mathrm{MW}$ installed worldwide. ${ }^{105}$ Due to the higher operating temperature, they have higher tolerance to impurities. The fuel cell efficiency can be improved to nearly 75 percent by using it in the combined heat and power (CHP) mode. Molten carbonate fuel cells (MCFC) operate at between 600 and $700^{\circ} \mathrm{C}$.

Regenerative options for PAFC and MCFC technology have not been explored. Solid oxide fuel cells (SOFC) operate in the 700 to $1,000^{\circ} \mathrm{C}$ range, with a stack size in the $1 \mathrm{~kW}$ to $2 \mathrm{MW}$ range. The round-trip efficiency is expected to be 36 percent for regenerative SOFC systems. For the SOFC and MCFC systems, the fuel cell efficiency by recovering waste heat is expected to be as high as 90 percent.

\footnotetext{
103 Telephone conversation with Dr. Vani Dantam, senior vice president of Axion Power, February $15,2012$.

${ }^{104}$ Email received by Viswanathan from Vani Dantam on February 16, 2012.

${ }^{105}$ http://www.fossil.energy.gov/programs/powersystems/fuelcells/fuelscells_phosacid.html
} 
Based on the above analysis, it does not seem likely that regenerative fuel cell systems will be deployed any time soon in wind-related applications. While the round-trip efficiency is low, the specific energy is estimated to be between 400 to $1,000 \mathrm{Wh} / \mathrm{kg}$, which is significantly higher than other energy storage systems. ${ }^{106}$ Lynntech, Hydrogenics Corporation and Proton Energy Systems are developing Unitized Regenerative Fuel Cells (URFCs).

Graphical representation of the schematic for a unitized regenerative fuel cell can be found in ( Rabih et al. 2008), while Figure 32 shows the schematic for a regenerative fuel cell with a separate electrolyzer and fuel cell. ${ }^{107}$ Additional information on the progress in the development of regenerative fuel cells is provided in Appendix 24 (Section 2.1).

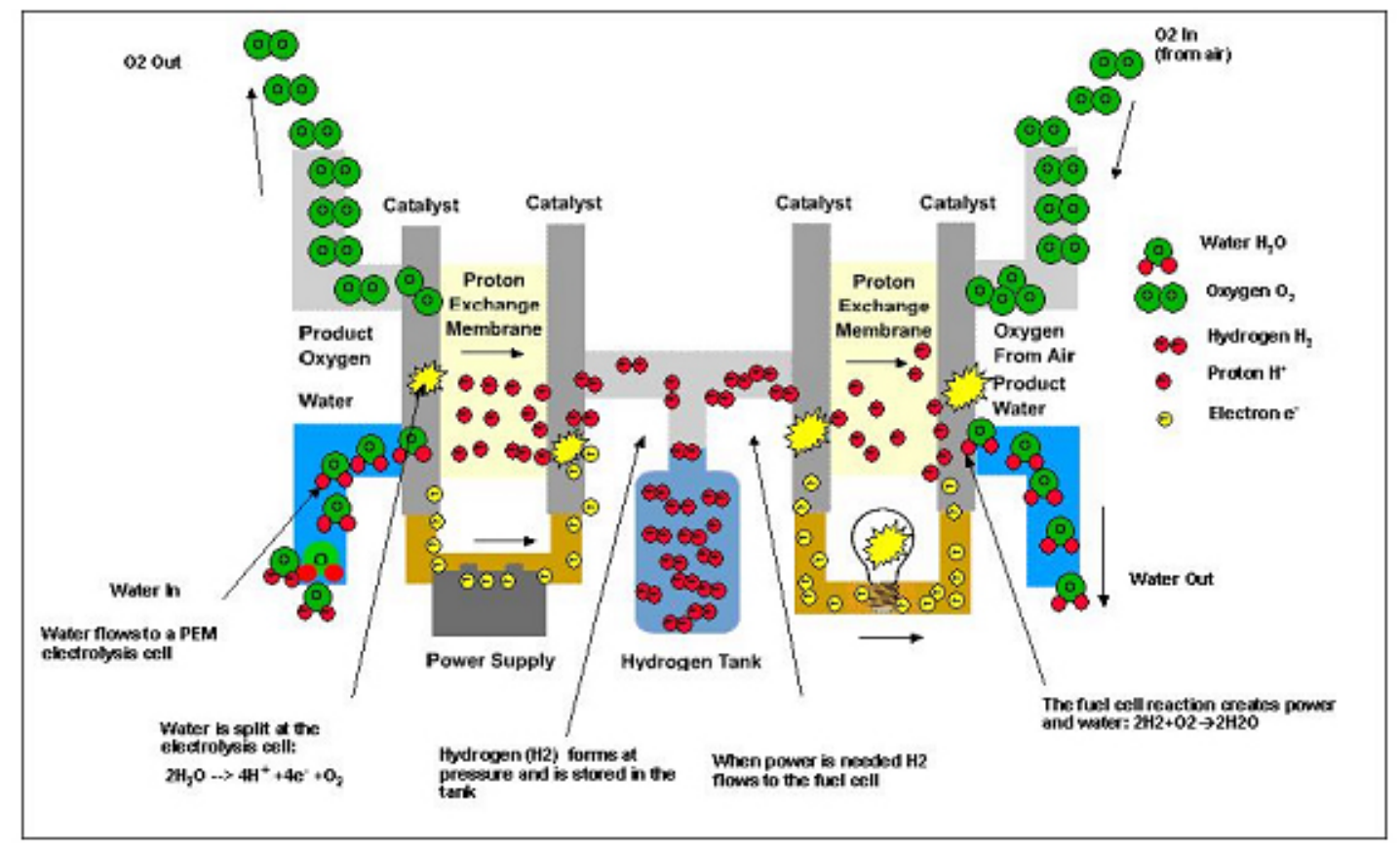

Figure 32. The Infinity XStorra regenerative fuel cell. ${ }^{108}$

\section{Baseload generation with fuel cells}

FuelCell Energy, Inc. is partnering with Abengoa S.A. to develop localized, stationary fuel cell power plants in Europe and Latin America. ${ }^{109}$ The technology consists of 300-kW molten carbonate direct fuel cell (DFC) modules provided by FuelCell Energy, while the balance of plant is developed by Abengoa. The initial pilot installation will be at the Abengoa headquarters in Palmas Campus Palmas Altas, Spain. The modules can provide a few megawatt of power, and will target municipalities, large industrial power users, and facilities that generate biogas to fuel the power plants. Ninety percent efficiency is expected in the CHP mode, with part of the

\footnotetext{
${ }^{106}$ http://www.machine-history.com/URFC\%20Unitized\%20Regenerative\%20Fuel\%20Cell

${ }^{107}$ http://infinityfuel.com/technology.htm

108 http://infinityfuel.com/technology.htm. Reprinted with permission from Infinity Fuel Cell and Hydrogen Inc.

109 http://www.fuelcelldispatch.com/StationaryPower/tabid/2703/articleType/ArticleView/articleId/4415/FuelCellEnergy-Surges-on-Plans-to-Supply-Biogas-Plants.aspx
} 
heat used to generate biogas from waste. High-temperature fuel cell systems, used in CHP mode, are a good candidate for URFC systems because of their high, overall efficiency.

SOFCs cells powered by landfill methane from Bloom Energy (San Jose, Calif.) have been installed by eBay, with five "refrigerator-sized" fuel cells generating as much power as 3,000 solar panels on the data center's roof (Johnson 2011). Federal Express, Google, Staples, and Walmart also have installed similar-sized Bloom Energy fuel cell systems.

InnovaTek is constructing a pilot SOFC system for CHP running on non-food bio oil with a steam reformer (Irving 2011). A field trial is planned for grid integration in the Richland, Wash., renewable energy park. Acumentrics Corp. has deployed a 3 to 10-kW SOFC system for integration to remote and micro-CHP platforms, with an electrical efficiency of 40 percent and an overall efficiency of 85 percent (Bessette 2011). They have modified their manufacturing process to decrease firing time by 50 percent, improve yield, and lower cost. The system has been in operation for 12,000 hours.

Intelligent Energy installed a proton-exchange, membrane fuel cell (PEMFC) system 40 miles southwest of London in a multiresidential housing development for CHP, operating at 50 percent efficiency with a six-month field trial scheduled to take place (Swamy 2011). PEMFC systems from United Technologies Corp (UTC) have been installed in fuel cell buses at three transit agencies, with a 50 to 130 percent improvement in efficiency over buses fueled by diesel and compressed natural gas (CNG) (Eudy 2011). These buses have logged 10,000 to 20,000 hours.

Additional examples of fuel cells used in various stationary applications are given in Appendix 24 (Section.2.2).

\subsubsection{High-temperature sodium sulfur batteries}

"NaS batteries have attractive energy density (over four times that of lead-acid battery) and low capital cost. They also have a long-cycle capability (2,500 plus cycles upon reasonable depth of discharge) and millisecond response with full charge and discharge, which have a good potential for regulation application" (Makarov et al. 2008c).

"The technology is highly efficient by having high-cell, DC efficiency (up to 89 percent), no self discharge, minimal maintenance, and long life (up to 15 years). NaS batteries are made of abundant, low-cost materials, which are suitable for high-volume mass production. Modular fabrication yields potentially high power and energy capability, which also reduces the construction intervals" (Makarov et al. 2008c).

"The NaS battery has a long cycle life, attractive energy density, and a good scaling potential to power system applications. However, there are some technical issues that still need to be properly addressed, particularly those related to the containment of the liquid electrodes (corrosion of the electrode containers and brittle glass seals)" (Makarov et al. 2008c).

The NaS battery, operated in the $250^{\circ} \mathrm{C}$ to $300^{\circ} \mathrm{C}$ range, has molten electrodes and a solid electrolyte. The anode is molten sodium ( $\mathrm{Na}$ ), the cathode molten sulfur (S) and the electrolyte a beta-alumina ( $\beta$ "-alumina) solid electrolyte (BASE). The beta-alumina in combination with a mobile sodium ion $\left(\mathrm{Na}^{+}\right)$transports sodium ions very well. During discharge, sodium ions at the anode migrate through the electrolyte to the cathode, while the electrons, travel externally to the cathode, where they combine with sodium ions and sulfur, and form sodium polysulfide $\left(\mathrm{Na}_{2} \mathrm{~S}_{\mathrm{x}}\right)$. The NaS battery is usually cylindrical with the anode contained in the center of a BASE cylinder, 
and the cathode contained between the BASE cylinder and another larger cylinder, that also forms the battery's outer casing (Makarov et al. 2008c). A schematic of a Na-S cell is given in Figure 33 below (Oshima and Kajita 2004).

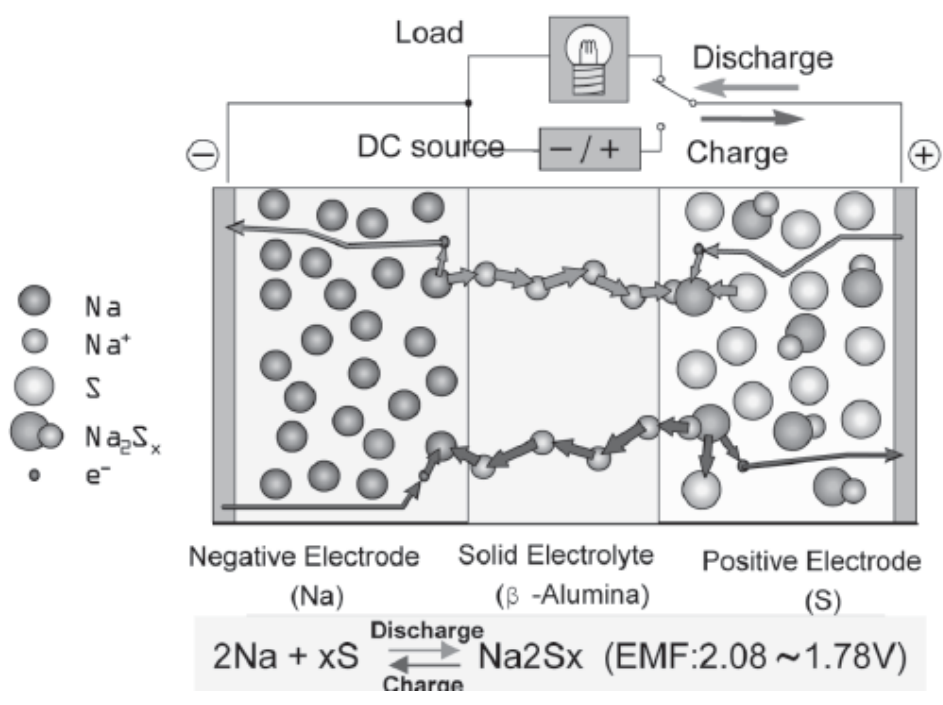

Figure 33. Schematic of the working principle of a NaS cell (Oshima and Kajita 2004). ${ }^{110}$

Sodium-sulfur ( $\mathrm{Na} \mathrm{S}$ ) batteries manufactured by NGK Insulators are in the commercialization stage, and are used in electric utility distribution grid support and wind power integration. As of 2010, 316 MW of Na S batteries were installed at 221 sites, representing 1,896 MWh, which included $50 \mathrm{MW}$ in Abu Dhabi (Rastler 2010). Na S batteries are only available in multiples of 1MW/6-MWh units, with installations typically in the range of 2 to $10 \mathrm{MW}$. The largest, single installation is the 34-MW Rokkasho wind-stabilization project in Northern Japan, which has been operational since August $1,2008 .{ }^{111}$ Agreements are in place to supply an additional $300 \mathrm{MW}$ to customers in Abu Dhabi and $150 \mathrm{MW}$ to Electricite de France over the next few years. Customers in the United States include American Electric Power (11 MW deployed), PG\&E (4 MW being deployed), New York Power Authority (1 MW, deployed) and Xcel Energy (1 MW, deployed). Installed capacity is anticipated to grow to $606 \mathrm{MW}$ (3636 MWh) by 2012 (Rastler 2010). Appendix 24 (Section 3.1) provides details on Na-S supply/demand as a result of a fire at one of the plants in Japan.

\subsubsection{Sodium metal halide battery}

The molten cathode in Na-S battery is very corrosive and tends to degrade the BASE electrolyte. Failure of the BASE electrolyte results in the liquid reactants penetrating the cracks and reacting violently upon contact. The semi-solid cathode of porous metal halide structure impregnated with $\mathrm{NaAlCl}_{4}$ in the Na-metal halide ZEBRA ${ }^{112}$ battery has a safer failure mode that results in a low-resistance cell to allow adjacent, series-connected cells to function. Use of alternative transition-metal chlorides is expected to lower operating temperatures, and to improve safety and reliability. The use of less-corrosive electrodes enables the use of thinner electrolytes, thus lowering internal resistance and component cost.

\footnotetext{
${ }^{110}$ Reused by permission of John Wiley \& Sons.

111 http://www.ngk.co.jp/english/products/power/nas/index.html

112 Zeolite Battery Research Africa Project.
} 
GE acquired Beta R\&D and in 2010, it marketed sodium-beta batteries based on Na-metal halide technology under the trade name Durathon. Eight kilowatt modules that were designed to discharge for two hours were built (Intrator et al. 2011). The markets being targeted are backup power supplies for hospitals, data centers, and wireless communication towers, where they compare with lead-acid battery technology. As grid-scale market grows, GE is expected to make a push into those markets. The competition between NGK Insulators Ltd. and GE technologies in terms of varying chemistry and module design is expected to improve performance and drive down costs in the near term. Additional information on the Zebra battery is provided in Appendix 24 (Section 3.2).

\section{Cost estimation for ZEBRA battery}

ZEBRA battery, produced by MES-DEA in Switzerland, consists of nickel, salt, and boehmite (Gallaway 2003). The worldwide nickel production is 1 million tons per year, with available resources of 200 million tones. INMETCO in Pennsylvania has recycled ZEBRA cells in 20-ton loads to produce nickel-containing alloy, which used in the stainless steel industry, and with the slag used in road construction. The material value covers the battery transportation and recycling process cost. For the battery system, the total cost was estimated to be $\$ 72.6 / \mathrm{kWh}$ for a 21-kWh system, assuming a fixed price of $\$ 250$ for the battery controller.

The high, specific energy makes it a good candidate for traction in electric buses, hybrid buses, and vans. The total material cost for a $38 \mathrm{Ah} 2.85-\mathrm{V}$ cell was estimated at $\$ 2.77$, corresponding to $\$ 28 / \mathrm{kWh}$. About 60 percent of the cost is for nickel, while iron (powder + sheet) comprises 17 percent and the beta-alumina ceramic electrolyte is 12.5 percent. Battery-grade nickel powder is used in the electrode and nickel sheet for the current collector, with the usage being 25 to 50 percent per kWh, compared to $\mathrm{Ni}-\mathrm{Cd}$ and $\mathrm{Ni}-\mathrm{MH}$ batteries. While the negative electrode is sodium, the cells are assembled in the discharged state using sodium chloride, which is very cheap at $\$ 0.3 / \mathrm{kg}$. The battery case is a double-walled, stainless-steel box. The cooling system and microporous foamed silica plates are located between the inner and outer walls. For a 21$\mathrm{kWh}$ battery, the estimated cost for the case is $\$ 9.4 / \mathrm{kWh}$, with close to 50 percent corresponding to thermal insulation and 28 percent corresponding to the stainless-steel case.

For the battery system, assuming energy costs for ceramic production is $\$ 1.7 / \mathrm{kWh}$ and cell material is 70 percent of total cell fabrication cost, and battery assembly cost equals casematerial cost, the total cost is $\$ 72.6 / \mathrm{kWh}$ for a $21-\mathrm{kWh}$ system, assuming a fixed price of $\$ 250$ for the battery controller. More details of the cost analysis are presented in Appendix 24

(Section 3.2.1).

\subsubsection{Nickel-cadmium (Ni-Cd) batteries}

"The nickel-cadmium battery is another electrochemical battery type, with a widespread use in portable consumer electronics. It is like the lead-acid battery in an electrochemical cell, based on the conversion of cadmium $(\mathrm{Cd})$ to cadmium hydroxide $(\mathrm{Cd}(\mathrm{OH}) 2)$ at the anode, and nickel oxyhydroxide $(\mathrm{NiOOH})$ to nickel hydroxide $(\mathrm{Ni}(\mathrm{OH}) 2)$ at the cathode. The electrolyte is not consumed by the process and is commonly an aqueous solution of potassium hydroxide (KOH). Compared with the lead-acid battery, the Ni-Cd battery has a higher energy density and is more temperature tolerant. For the purpose of regulation, it has the important feature of being tolerant to deep discharges, and storage during the discharged state. Its drawbacks are higher costs, and the need for advanced battery monitoring during charge and discharge" (Makarov et al. 2008c). 
An example for the stationary deployment of $\mathrm{Ni}-\mathrm{Cd}$ batteries is the Golden Valley Electric Association BESS (battery energy storage system) (Golden Valley Electric Association, undated) project in Alaska. The batteries were supplied by SAFT and the power electronics by ABB. This system, commissioned in December 2003, was designed to provide $26 \mathrm{MW}$ for 15 minutes, or a full $40 \mathrm{MW}$ for 7 minutes.

\subsubsection{Nickel metal hydride (Ni-MH) battery}

"The nickel metal hydride (Ni-MH) battery is a variant of the Ni-Cd battery, having an alloy of various rare metals as the anode instead of cadmium. It shares many advantages with the Ni$\mathrm{Cd}$ battery, including robustness, deep discharges and a long cycle life. In addition, it has a higher energy density than $\mathrm{Ni}-\mathrm{Cd}$ batteries, but also a slightly larger internal resistance.

The application of $\mathrm{Ni}-\mathrm{MH}$ batteries is widespread in consumer electronics, replacing the $\mathrm{Ni}-\mathrm{Cd}$ as the preferred substitute of alkaline cells in cameras, toys, and other batter-powered items. On the kW scale, it is found in most hybrid electric vehicles currently on the market. However, there is no operating experience on the megawatt scale for electric utility storage" (Makarov et al. 2008c).

\subsubsection{Nickel-zinc battery}

This battery has the potential to have high, specific energy and low cost compared to Ni-Cd. However, issues related to zinc dendrite growth and zinc electrode shape change need to be resolved. The cycle life is poor, and therefore it is not a viable candidate for stationary applications. Pavlov et al. (1996) got more than 500 cycles for a 44 Ah Ni-Zn battery by using a thin, high-porous nickel membrane (HPNM) between the electrodes. Essentially, nickel reacts with oxygen to form $\mathrm{Ni}(\mathrm{OH}) 2$, which dissolves the zinc dendrite by forming a Ni-Zn pair. Batteries without the HPNM gave only 100 cycles.

Energy Research Corporation (ERC) obtained over 600 cycles at 80 percent DOD at C/3 discharge rate. The negative has three layers of Celgard 2400 and two layers of Pellon (U.S. Department of Energy 2010). The positive is wrapped in three layers of Celgard, while the zinc electrode is wrapped in three layers of pellon. This can lead to a high internal resistance. ERC also developed a 2.2Ah Ni-Zn battery for a left ventricular assist system (U.S. Department of Energy 2010). The prismatic cell had a spring-loaded vent designed to operate at $270 \mathrm{kPa}$, with cell case burst pressure of $760 \mathrm{kPa}$. The main mode of cell failure was dry-out because of an unoptimized charge protocol. Cao et al. described the current status of their Ni-Zn batteries. Model 40 (30 Ah 7-cell 12-V battery) gave 600 cycles at 100 percent DOD (Cao et al. 2000). They had a high-rate, fast-charge system using taper charge with three steps. The first cell failure occurred after 525 cycles at 100 percent DOD. Cell disassembly showed no defects, zinc dendrites or shape change. The mid-point discharge voltage decreased linearly with cycles up to 550 cycles, indicating dry-out as the failure mode. Additional details of Ni-Zn battery management are given in Appendix 24 (Section 5).

\subsubsection{Silver-zinc batteries}

$\mathrm{Ag}$-Zn batteries have a high specific energy and energy density (Coates et al. 1997, Karpinski et al. 1999, Karpinski et al. 2000, Lewis and Salkind 1997, Himy 1986, Dalin et al. 1964) with silver typically the capacity-limiting electrode. Hence, on overcharge, oxygen is evolved at the positive. The oxygen diffuses to the negative, and recombines with metallic zinc to form zinc oxide, which is converted to zinc. The recombination rate for oxygen at the zinc electrode is 
about eight times higher than that for hydrogen at the silver electrode. Therefore, it is important that hydrogen evolution be avoided during overcharge.

On normal discharge, very little gas is generated. On cell reversal, hydrogen is evolved at the positive, followed by oxygen evolution when negative is depleted. Excessive overdischarge may result in venting or cell case rupture. If there is some electrode protrusion above the electrolyte, some of the oxygen and hydrogen evolved may recombine at the opposite electrode.

Additives are incorporated to minimize degradation. Zinc electrodes undergo a shape change caused by solution and reprecipitation of zinc oxide as the cell cycles between charge and discharge. Teflon additive was effective in reducing this shape change, but reduced the oxygen recombination rate. The zinc electrode self-discharge was suppressed by raising the hydrogen overvoltage by adding mercury. Mercury also was effective in controlling the growth of zinc dendrites.

Zn electrode degradation occurs as a result of gassing and shape change, resulting in higher polarization losses. There also is some evidence of silver oxalate formation at the positive electrode, which can grow in size and rupture the separator. Cellophane separator layers get oxidized by the silver compounds, reducing them to silver. Zinc dendrite growth results from deposition of zinc in areas of high current density, and growth of the zinc deposits away from the negative electrode. Dendritic growth through the separator does not occur by mechanical perforation, but by diffusion. Hence, a separator that resists zincate diffusion would limit dendritic growth. Until these issues are resolved, in spite of its high specific energy, Ag-Zn does not appear to be a viable option for stationary applications.

\subsubsection{Metal-air batteries}

"With high energy density as its principal asset, the metal-air battery technology does not have a manufacturing base that is well developed enough to be a candidate for utility-scale operations. The drawbacks for power system applications are severe, and include complicated recharging (many types are not electrically rechargeable, but must have active material replaced), low power output, and poor cycle life" (Makarov et al. 2008c).

Metal-air batteries couple a reactive anode to an air electrode. The anode candidates ate $\mathrm{Li}, \mathrm{C}$, $\mathrm{Mg}, \mathrm{Al}, \mathrm{Zn}$ and Fe. Most metal-air batteries use alkaline electrolyte. The metal-air batteries have a high energy density, flat discharge voltage and a long shelf life if stored dry. These batteries are environmentally friendly and low cost. Table 9 shows the specific energy for various metal anodes (Linden 1995).

Table 9. Properties of various metal-air chemistries ( ${ }^{*}$ actual Open Circuit Voltage (OCV). ${ }^{113}$

\begin{tabular}{|l|l|l|l|l|l|}
\hline Anode & $\begin{array}{l}\text { Theoretical } \\
\text { Ah/g }\end{array}$ & $\begin{array}{l}\text { Theoretical } \\
\text { cell voltage } \\
(\mathrm{V})\end{array}$ & $\begin{array}{l}\text { Valence } \\
\text { change }\end{array}$ & $\begin{array}{l}\text { Theoretical } \\
\text { specific } \\
\text { energy } \\
(\mathrm{Wh} / \mathrm{kg})\end{array}$ & $\begin{array}{l}\text { Operating } \\
\text { voltage } \\
(\mathrm{V})\end{array}$ \\
\hline $\mathrm{Li}$ & 3.86 & $3.4(2.85)^{\star}$ & 1 & 13.0 & 2.4 \\
\hline $\mathrm{Ca}$ & 1.34 & 3.4 & 2 & 4.6 & 2.0 \\
\hline $\mathrm{Mg}$ & 2.20 & $3.1(1.6)^{\star}$ & 2 & 6.8 & $1.2-1.4$ \\
\hline $\mathrm{Al}$ & 2.98 & 2.7 & 3 & 8.1 & $1.1-1.4$ \\
\hline $\mathrm{Zn}$ & 0.82 & 1.6 & 2 & 1.3 & $1.0-1.2$ \\
\hline $\mathrm{Fe}$ & 0.96 & 1.3 & 2 & 1.2 & 1.0 \\
\hline
\end{tabular}

${ }^{113}$ Adapted from Table 38.2, Handbook of Batteries $2^{\text {nd }}$ edition David Linden. 
Aluminum, iron, magnesium, and lithium suffer from severe corrosion problems. Al-air and Mgair cells operated either as reserve systems or as mechanically rechargeable batteries with anode replacement. Iron electrodes are pyrophoric and need to be protected from exposure to air. Safety is a big issue for aqueous Li-air batteries because the anode has to be protected from moisture.

Electric Fuel Corporation developed mechanically rechargeable batteries, where the zinc anode was replaced.

Cell sizes of 2.9 Ah and 4.5 Ah were developed with a pack voltage of 3.6, 4.8 and 6 Vfor cell phone.

Zn-air batteries (or fuel cells) with mechanical recharging have been used to power buses and for stationary power generation. Each cell comprises a thin-air cathode with a nonprecious metal catalyst deposited over a carbon current collector, a polymer membrane separator, an alkali electrolyte, and a powdered zinc anode. Currently, Fluidic Energy is developing zinc-air batteries with an ionic liquid electrolyte and a proprietary catalyst for utility-scale applications (Yang et al. 2010).

While using atmospheric oxygen improves specific energy, this also puts a limit on the discharge rate because of limitations in oxygen ingress to the cell. Also, catalysts with high activity for oxygen reduction and evolution need to be developed. Clogging of catalyst pores with the discharge product at the cathode needs to be mitigated. Uniform plating of zinc or lithium onto the anode is another challenge that needs to be addressed. For Zn-air cells, chelating ligands have been developed to facilitate uniform zinc plating.

Eos Energy Storage has developed a zinc-air battery technology that has provided $>2,000$ cycles with no significant deterioration. ${ }^{114}$ The estimated capital cost is $\$ 1,000 / \mathrm{kW}$ (or $\$ 160 / \mathrm{kWh}$ for 6-hour storage), with building blocks of $17 \mathrm{~kW} / 1,000 \mathrm{kWh}$. The use of non-aqueous electrolyte with near-neutral $\mathrm{pH}$ mitigates the clogging of the air electrode as a result of carbonate formation from $\mathrm{CO}_{2}$ absorption onto potassium hydroxide $(\mathrm{KOH})$ electrolyte. They claim that the electrolyte prevents zinc dendrite formation and allows operation without a membrane. This also helps in avoiding zinc electrode shape change during cycling. Incorporation of additives also avoids zinc plating, while an electrolyte management system prevents dry-out. No modules have been installed yet, but MW-scale systems are expected to be delivered in 2013. ${ }^{115} \mathrm{~A}$ round-trip efficiency of between 72 and 75 percent is anticipated for the system. A summary of Zn-air batteries developed in the past is provided in Appendix 27 (Section 4).

In the area of Li-air primary batteries, using optimized cathode architecture, electrolyte, and packaging membrane with oxygen permeability and moisture blocking property, researchers at PNNL have achieved a specific energy of $362 \mathrm{Wh} / \mathrm{kg}$ - based on the total battery weight, which is an improvement of almost double that of state-of-the-art lithium-ion batteries (Zhang et al. 2010). Using novel graphene-based air electrode, the discharge capacity can be as high as 15 Ah/g-carbon, which is the highest capacity reported for $\mathrm{Li}^{-\mathrm{O}_{2}}$ batteries (Xiao et al. 2011). Abraham built the first Li-air battery in 1996, and manufacturers built Li-air batteries with high rechargeability 10 years later (Abraham and Jiang 1996). To date, manufacturers have not

\footnotetext{
${ }^{114} \mathrm{http}: / /$ www.eosenergystorage.com/technology, accessed February 3, 2012.

${ }^{115}$ Email from Michael Binder of Eos, received February 6, 2012.
} 
developed Li-air batteries for the commercial market (Armand and Tarascon 2008). A schematic of a Li-air cell is shown in Figure 34.

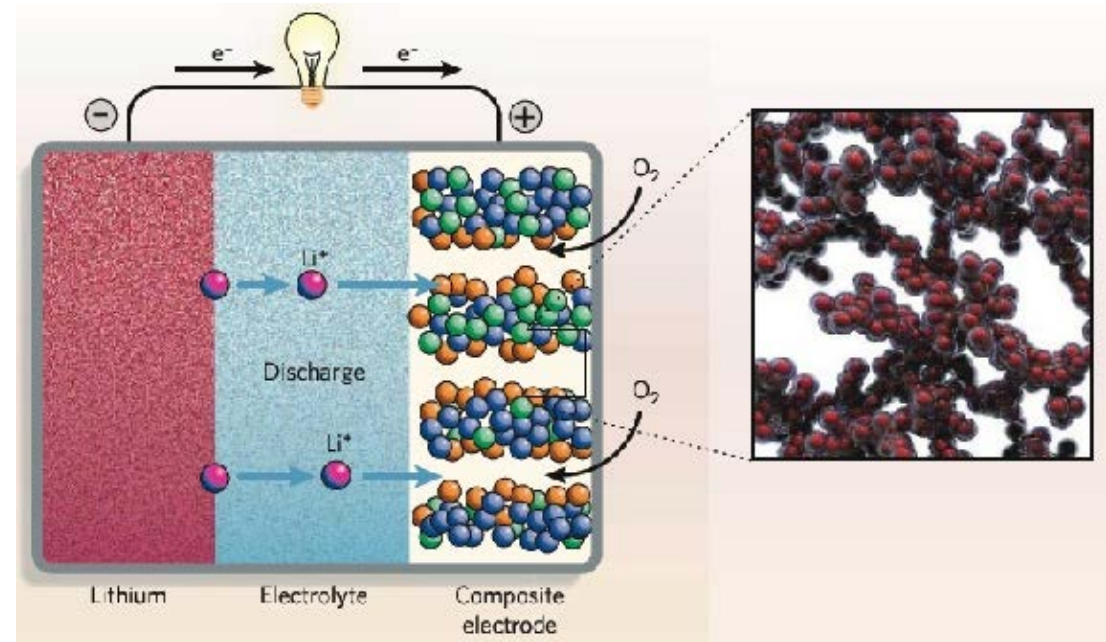

Figure 34. Schematic of a Li-air cell (U.S. Department of Energy 2010).

\subsubsection{Lithium-ion battery}

"With high-energy density and long-cycle life, lithium-ion batteries are increasingly becoming the workhorse battery in portable consumer electronics, but they are not yet suitable for bulk power storage at the utility scale" (Makarov et al. 2008c).

"The lithium-ion battery is like nickel and lead-acid batteries, based on an electrochemical process, but they are unlike these batteries in that the electrolyte is non-aqueous. The battery consists of two electrodes, both containing lithium, submerged in an electrolyte, which is a lithium salt in an organic solvent, e.g., ether. The anode is usually graphite $\left(\mathrm{LiC}_{6}\right)$ and the cathode lithium cobalt oxide $\left(\mathrm{LiCoO}_{2}\right)$. During discharge, lithium ions ( $\mathrm{Li}+$ ) migrate from the anode, through the electrolyte, to the cathode. An electron similarly moves from the anode to the cathode, outside the battery, creating an electric current" (Makarov et al. 2008c).

Some drawbacks, however, limit the application of lithium-ion batteries. In the charged state, the battery is extremely sensitive to over-temperature, over-charge, and internal pressure build-up, thus requiring advanced battery management systems. The Li-ion battery also has a limited calendar life (Makarov et al. 2008c). These limitations are being addressed with safer cathodes, such as lithium iron phosphate $\left(\mathrm{LiFePO}_{4}\right)$. Details on novel cathode and anode development are provided in Appendix 24 (Section 6.1).

Additional details on Li-ion batteries are given in Appendix 24, Section 6. Li-ion batteries are mainly used in the regulation market, where the discharge time is about 15 minutes. Shown below in Figure 35 are some battery systems from various manufacturers: 


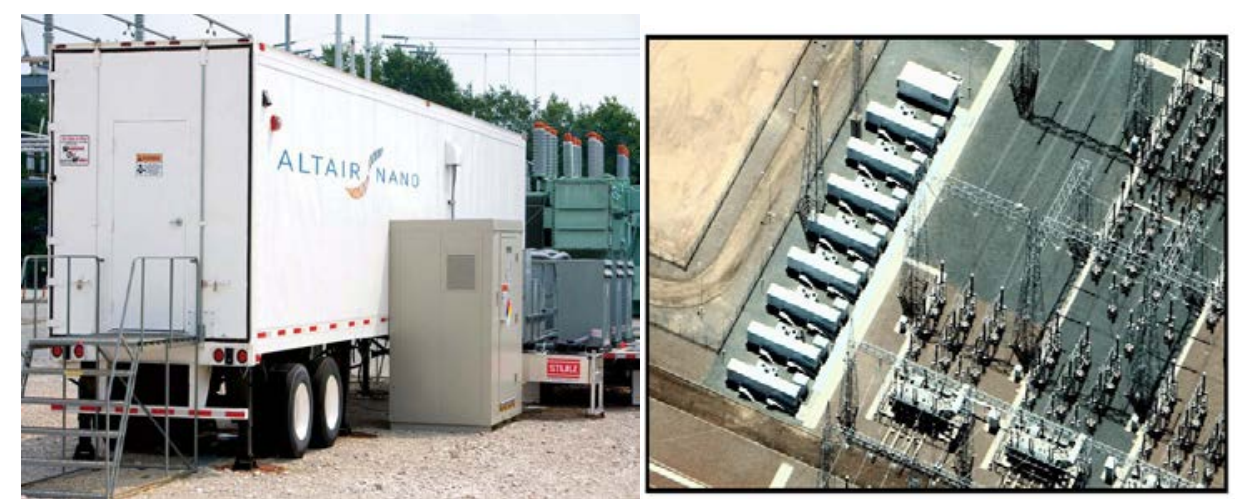

Figure 35. (left) 1-MW/250-kWh mobile Altairnano Li-ion energy storage system deployed at PJM; and (right) A123 Systems' 12-MW Li-ion Hybrid Ancillary Power Units (Hybrid-APUs ${ }^{\mathrm{TM}}$ ), installed by AES in Chile substation (Rastler 2010).

\subsubsection{Flow batteries}

"Flow batteries pump catholytes and anolytes through the cell, with additional electrolyte stored outside the battery in tanks (Makarov et al 2008c). The cells are connected in a series to form stacks, which in turn are connected in a series (or parallel configuration) to form modules (batteries). In redox (reduction-oxidation), flow batteries, such as the vanadium redox battery (VRB) and the polysulfide bromide battery (PSB or Regenesys), all chemicals that actively take part in the electrochemical energy conversion are dissolved in the electrolyte, with no deposit of material onto the electrodes during operation. Therefore, the energy capacity is determined only by the size of the electrolyte tanks, whereas the power capacity is determined by the stack size and number of stack, along with pumping capacity. The ability to vary power and energy capacity is one of the major advantages of the redox batteries.

In contrast, the hybrid flow batteries, such as the zinc bromine $(\mathrm{ZnBr})$ batteries, are based on processes, where material is deposited as solids onto the anode during charging or discharging, while the catholyte flow through the cathode compartment (Makarov et al 2008c).

Common advantages of flow batteries are long cycle life, short response time and a symmetrical charge/discharge rate, making them suitable for power system applications. ${ }^{117}$ Particularly, the vanadium redox battery technology seems to be promising due to its high safety characteristics. But, present installations are mostly test sites or demonstration projects" (Makarov et al 2008c).

\subsubsection{Li-ion flow batteries}

24M Technologies, an A123 spinoff, has been awarded $\$ 2.5 \mathrm{M}$ by advanced research projects agency - Energy (ARPA-E) to develop liquid reactants, Cambridge Crude, for use in high-range electric vehicles (Johnson 2011). The company also has received $\$ 10$ million in Series $A$ financing from Charles River Ventures and North Bridge Venture Partners, bringing its total startup funding to $\$ 16 \mathrm{M}$. The goal is to produce batteries with a specific energy of $500 \mathrm{Wh} / \mathrm{kg}$ at a cost of $\$ 250 / \mathrm{kWh}$ and $\$ 100 / \mathrm{kWh}$ for transportation and grid applications, respectively. The reduction in cost can be achieved through lower cost for electrode fabrication because electrode coating, calendaring, slitting, tabbing, and module assembly steps can be eliminated. The

\footnotetext{
${ }^{116}$ Reused by permission of EPRI.

117 This symmetry is related to current. The charge power will exceed discharge power at a fixed current; hence, the internal resistance needs to be minimized.
} 
researchers claim that compared to aqueous chemistries, the energy density of the liquid fuel is an order of magnitude higher. Carbon nanoparticle suspensions form an electronically conductive network for electron transport to and from the current collectors. This technology is also known as the semi-solid flow cell (SSFC). In tests performed to date, the energy density is much lower than Li-ion packs, while a two order of magnitude increase in the conductivity of the carbon nanoparticle network is needed for viability.

\subsubsection{Vanadium redox flow batteries}

Vanadium redox batteries are the most mature of all flow battery systems available. In flow batteries, energy is stored as charged ions in two separate tanks of electrolytes, one for each electrode. Changing the stack and tank size, respectively, can independently control the power and energy.

Redox flow batteries were developed in the 1970s and have gained recent prominence because of their flexibility (Herman 2003, Rastler 2010). Similar to a regenerative fuel cell, the power and energy components are separated, with the stack providing power and the electrolyte storage tanks providing energy. Vanadium redox flow batteries have been deployed with a wide range of power and energy capacity in the kW-MW and kWh-MWh models. The efficiency of these systems is in the 70 to 80 percent range, with response time in the order of milliseconds. The stacks last at least 10 years and can sustain $>200,000$ cycles. Active research is ongoing to reduce stack costs by addressing individual components and also by increasing the power density.

While most vanadium redox flow batteries operate in the 0 to $40^{\circ} \mathrm{C}$ temperature range, a wider $\left(10\right.$ to $50^{\circ} \mathrm{C}$ ) range has been demonstrated at PNNL (Li et al. 2011). Widening the temperature range would lower costs associated with heat exchangers, while also increasing the life of the electrolyte. Efforts are also ongoing to reduce stack costs by development of higher performance stacks, lower cost membranes, electrodes, and bipolar plates. On the energy side, higher concentration electrolytes are being developed to increase energy density. System design issues being addressed include minimization of losses associated with pumping and shunt current.

Vanadium redox systems can be designed to provide application varying energy/power ratio depending on the application.

\section{Performance and life}

The lifespan of flow-type batteries is not strongly affected by cycling. Suppliers of vanadium redox systems estimate lifespan of the cell stacks to be 15 or more years, while the balance of plant and electrolyte can have life times of over 25 years. Batteries have achieved cycling capability of 10,000 or more cycles at 100 percent depth of discharge.

The efficiency of the system is mainly a function of the power density, and can be controlled to be in the 75 to 85 percent range by varying the stack area for a desired power output. Hence, this provides an additional degree of freedom by designing the stacks appropriately for short duration applications, such as balancing and regulation, versus energy intensive applications, such as arbitrage and load leveling. The cycle life of redox flow batteries depends on its use profile. Tokuda et al. (2000) targeted at least 1,500 cycles over 10 years. In principle, the battery 
can be cycled an order of magnitude higher, as long as the charge voltage is maintained below the gas evolution range (Energizer Resources 2011, Energy Storage News 2010, V-Fuel Pty Ltd, 2008, Staudt undated). The Tomamae wind energy storage demonstration, using a 4MW/6-MWh Sumitomo Electric Industries system, has undergone $>200,000$ cycles after three years, thus indicating the life for VRB systems is limited mainly by calendar life rather than by cycle life (Skyllas-Kazacos undated). Additional details on vanadium redox battery capital and O\&M costs are given in Appendix 24 (Section 7.1).

\section{Existing projects}

Vanadium redox flow battery systems range from several small 5-kW units deployed in field trials (a 15-kW/120-kWh system deployed by Risø - DTU, Denmark, a 50-kW/200-kWh system installed by Kashima - Kita Electric Power in 1995, a 200-kW/800-kWh system installed by VRB at the King Island wind farm in Australia, a 250- kW/2-MWh system built by VRB Power Systems and installed by PacificCorp in Castle Valley, Utah), to the 4-MW/6-MWh system built by Sumitomo Industries at the 32-MW Tomamae wind farm in northern Japan (Rastler 2010, Eckroad 2007, Yang et al. 2011, Steeley 2005, Zhang 2009).

The physical scale of vanadium redox systems tends to be large because of the large volumes of electrolyte required when sized for utility-scale (megawatt-hour) projects. Shown below in Figure 36 is a 5-kW/30-kWh installation of a Prudent Energy redox flow battery system in Kenya (Rastler 2010).

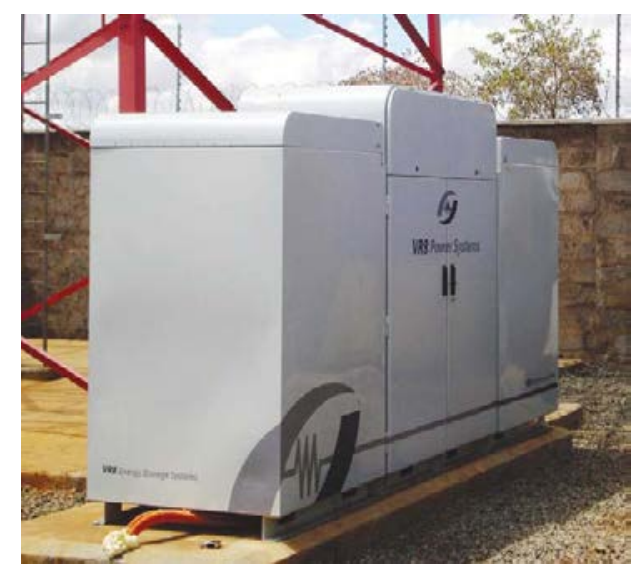

Figure 36. Prudent Energy 5-kW/30-kWh Vanadium Redox Flow Battery System installed at Kitangi, Kenya (Rastler 2010). ${ }^{118}$

\subsubsection{Zinc-bromine redox flow battery}

$\mathrm{Zn}-\mathrm{Br}$ batteries are less mature than vanadium redox flow batteries, and are in the early stages of field deployment and demonstration. In a zinc-bromine battery, anolytes and catholytes flow past carbon-plastic composite electrodes, with its compartments separated by a microporous polyolefin membrane. During a charge, metallic zinc is deposited on the carbon-plastic composite anode, while bromine evolves at the positive electrode, and reacts with organic amines to form a thick, bromine oil that sinks to the bottom of the electrolyte tank. During discharge, the bromine is mixed with the rest of the catholyte, which combines with zinc ions

\footnotetext{
${ }^{118}$ Reused by permission of EPRI.
} 
that cross over to the catholyte to form zinc bromine. The overall coulombic efficiency is 75 percent, which is low.

Module sizes range from $5 \mathrm{~kW}$ to $500 \mathrm{~kW}$, with energy storage ranging from two to six hours. In rural Australia, 5-kW/10-kWh systems have been deployed in lieu of new power lines. In the U.S., utilities have been planned to conduct trials of a Premium Power 0.5-MW/2.8-MWh transportable systems for grid support and reliability, with deployment by EPRI (2011) and a consortium of electric utilities. The vendors claim 20-year lifetime, long-cycle lives, and an ACAC efficiency of between 65 and 70 percent Rastler (2010). Some examples of installations are shown in Figure 37.

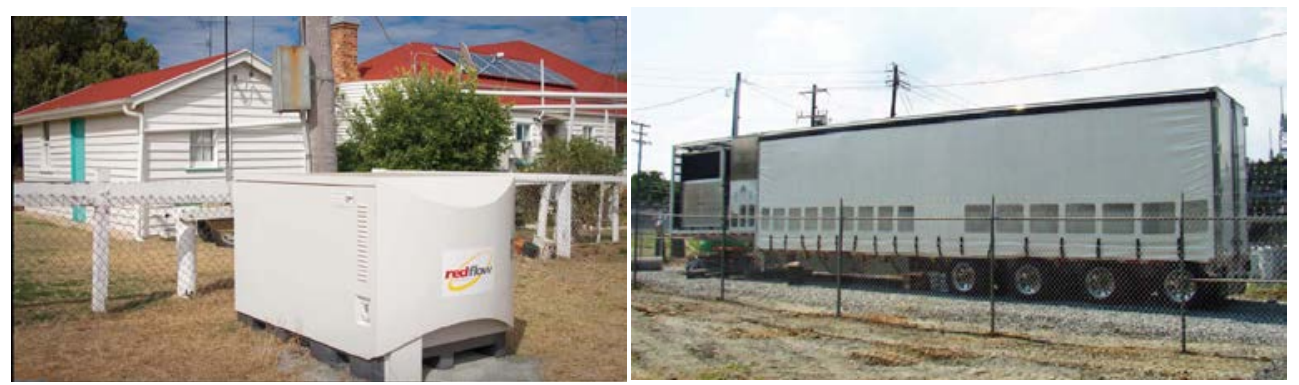

Figure 37. (left) 5-kW/20-kWh RedFlow Power community energy storage systems. (right) Premium Power's 0.5-MW/2.8-MWh TransFlow 2000 Transportable zinc-bromine energy storage system (Rastler 2010). ${ }^{119}$

An assessment of RedFlow Ltd. zinc-bromine battery, funded by RedFlow, was performed recently by Garth Corey (2010). The key points from this report were:

- Turnkey systems have been developed based on the RedFlow ZBM battery building block;

- The initial system was a 5-kW/10-kWh unit to verify operability and perform troubleshooting;

- The engineering challenges appear to have been addressed and solved;

- The ZBM building block is close to commercialization;

- Ongoing work is being done with utility customers to install a fully functional, gridconnected energy storage system;

- Future plans include development of a manufacturing capability;

- Shunt current losses have been minimized and battery management optimized to remove need for active cooling; and

- Use of a low-cost pump with high efficiency and reliability.

RedFlow provides a turnkey system with battery management system (BMS) in place to prevent battery abuse and premature failure. The RedFlow M120 120-kVA, 240-kWh, is designed to store off-peak electricity for time shifting. The system consists of 24 5-kVA modules, with 120 kVA continuous rating and $240 \mathrm{kWh}$ of energy storage, and weighs $16,000 \mathrm{~kg}$ with battery refurbishment needed after 1500 cycles. Smaller 10 to 20-kWh systems have been developed

\footnotetext{
${ }^{119}$ Reused by permission of EPRI.
} 
for residential use and for remote areas to manage peak demand and intermittent renewable generation. Appendix 24 (section7.2.1) provides additional information on RedFlow battery systems.

A ZBB Energy Corporation battery was tested for grid support at the Detroit Edison test sites using a 200- kW/250-kVA power conditioning systems (PCS) provided by SatCon Power Systems. The project was managed by Sandia National Laboratory and funded by U.S. DOE (Scaini et al. 2002). Graphical representation of ZBB 200 kW, 400 kWh zinc bromine energy storage system can be found in (Scaini et al. 2002). The building block is a 25-kW/50-kWh module, with the battery system energy being $400 \mathrm{kWh}$ rated at two hours (or $200 \mathrm{~kW}$ ). The battery/PCS system was successfully used for VAr support and for peak shaving.

ZBB is targeting utility load management (peak shaving and load leveling), solar and wind energy storage, emergency backup power, uninterrupted power supply (UPS), EV, golf cart, and wheelchairs (Scaini et al. 2002, Thomas 2003). They have a "repeatable manufacturing technique" that uses low-cost materials and "advanced vibration welding technology" at their Milwaukee pilot production line. Among its features are 2000 cycles at 100 percent DOD, high specific energy (65-84 Wh/kg), and wide operating temperature without degradation, modularity and the use of mostly plastic materials that simplifies production. Several demonstration projects with energy storage capacity up to $500 \mathrm{kWh}$ have been performed (Scaini et al. 2002, Thomas 2003, Norris et al. 2002). Additional details on demonstration projects for ZBB systems, along with capital and commissioning costs, are provided in Appendix 24 (Section 7.2.2).

Premium Power, based in North Andover, Mass., has three energy storage products: TransFlow 2000, Powerblock 150, and Zinc Flow 45. ${ }^{120}$ The TransFlow 2000, $500 \mathrm{~kW} / 2.8 \mathrm{MWh}$, is a fully integrated system that consists of energy storage, power conditioning, system control, and thermal management subsystems. The turnkey system is loaded onto a 53-foot trailer that can be dispatched for online storage as needed. The PowerBlock 150 provides $100 \mathrm{~kW}$ of continuous power and $150 \mathrm{kWh}$ of turnkey, transportable energy storage. The main application is power quality, where they deliver two to three times more energy than lead-acid battery UPS systems. The Zinc-Flow 45 provides instantaneous standby power for telecommunications, cable communication, data communications, and utility substation control sites. It is housed in a rain-tight enclosure that allows outdoor or indoor installation, with installation time less than four hours. Remote monitoring and control is enabled.

Table 10 compares these systems:

${ }^{120}$ www.premiumpower.com 
Table 10. Comparison of the various Premium Power modules. ${ }^{121}$

\begin{tabular}{|c|c|c|c|}
\hline & TF2000 & Powerblock 150 & Zinc-Flow 45 \\
\hline Energy (kWh) & 2800 & 150 & 45 \\
\hline Rated Power (kW) & 500 & 100 & 30 \\
\hline Voltage in/out & $480 \mathrm{VAC}, 60 \mathrm{~Hz}$ & $480 \mathrm{VAC}, 60 \mathrm{~Hz}$ & \\
\hline Weight & $\begin{array}{l}43,545 \mathrm{~kg} \text { (including } \\
\text { electrolyte and trailer) }\end{array}$ & $\begin{array}{l}4,000 \mathrm{~kg} \text { (includes } \\
\text { enclosure) }\end{array}$ & $\begin{array}{l}1622 \mathrm{~kg} \text { (includes rain- } \\
\text { tight enclosure) }\end{array}$ \\
\hline Safety & $\begin{array}{l}\text { UL } 1742, \text { FCC Part } 15, \\
\text { Class A, NFPA } 1 \& 70\end{array}$ & $\begin{array}{l}\text { UL } 1778, \text { FCC Part } 15 \\
\text { Class A, NFPA } 1 \& 70\end{array}$ & $\begin{array}{l}\text { UL 1778, FCC Part } 15 \\
\text { Class A, NFPA } 1 \& 70\end{array}$ \\
\hline Applications & $\begin{array}{l}\text { Substation T\&D asset } \\
\text { deferral, distribution } \\
\text { system load support, } \\
\text { peak shaving, demand } \\
\text { response management, } \\
\text { high quality power } \\
\text { output, energy arbitrage, } \\
\text { ancillary services, and } \\
\text { time shifting }\end{array}$ & $\begin{array}{l}\text { Power quality for utility, } \\
\text { renewable for } \\
\text { electronics, computer } \\
\text { network, } \\
\text { communications, and } \\
\text { manufacturing } \\
\text { environments }\end{array}$ & $\begin{array}{l}\text { Utility T\&D, remote } \\
\text { power applications, } \\
\text { instantaneous standby } \\
\text { power for } \\
\text { telecommunications, } \\
\text { cable, and data }\end{array}$ \\
\hline
\end{tabular}

\subsubsection{Flywheels}

This section addresses advantages and disadvantages of flywheels and their current status.

\subsubsection{Physics}

Flywheels consist of rotors made of steel or carbon composites, and store energy in the form of kinetic energy associated with the rotor speed. The motor/generator set is coupled to the same shaft to which the rotors are attached, with rotation occurring in a vacuum to minimize losses. The rotors can be supported by passive or electro-magnetic bearings, with high-temperature, superconducting, magnetic bearings requiring cryogenic cooling providing the best performance (Bolund 2007). While rotors made of graphite fiber composites currently are used, carbon nanotubes are expected to increase the energy density by an order of magnitude over carbon fiber winding (Sibley undated).

\subsubsection{Advantages and disadvantages}

"This is an established modular technology that has a proven growth potential to utility-scale with no significant "show-stopper" reservations. The flywheel energy storage is a "green" technology that has long cycle life and is insensitive to the depth of discharge" (Makarov et al 2008c).

"Besides, high-peak power capacity without overheating concerns, high, round-trip energy efficiency, rapid response, the ability to provide other ancillary services because of power

121 Telephone conversation between M. Lazarewicz of Beacon Power with Viswanathanon September 12, 2011. 
electronic interface with the grid, and the ability to site wherever needed, are strong positive values of the technology" (Makarov et al. 2008c). The efficiency of the flywheels systems ranges from 85 to 90 percent, while their ramp rate is as high as 100 percent-rated power per minute.

\subsubsection{Current status}

The major manufacturers of flywheels have been listed elsewhere (Gyuk and Eckroad 2003), with about half the manufacturers using steel rotors, which is appropriate for low-energy, powerquality applications. Among the current manufacturers, Beacon Power has developed flywheels that can provide $25 \mathrm{kWh}$ at a power of $100 \mathrm{~kW}$, and is currently developing 100-kW/100-kWh modules. ${ }^{122}$ Flywheel systems using such modular flywheels have been developed to provide 20-MW power and an energy of $5 \mathrm{MWh}$, with a goal of $100 \mathrm{MWh}$. For this module, the overall efficiency was 85 percent, with 13 percent losses attributed to the PCS 40-kWh systems running at 25-kW peak power; and 4-kW continuous power developed by Tribology Systems Inc. have ceramic bearings, with an estimated energy loss of $<0.03$ percent per hour, allowing the flywheels to operate unpowered for over four months (Sibley undated). ${ }^{123}$ Velkess has developed 10-kW/80-kWh systems targeting telecommunications (Gray 2009).

\subsubsection{Flywheels performance and life}

Flywheels are expected to last 25 years, with a cycle life of 125,000 at 100 percent DOD (Sibley undated). ${ }^{124}$ While DOD typically determined cycle life for batteries, for flywheels, the wear and tear mainly depends on the rotational speed of the rotors. Hence, the number of charges to 100 percent state of charge (SOC) is expected to be more degradative compared to number of charges to 50 percent SOC. For example, the cycle life at 25 percent DOD was estimated to be 450,000 by Beacon Power. The efficiency ranges from 85 to 95 percent. Additional details on system efficiency and demonstrations are provided in Appendix 24 (Section 9).

Flywheels capital and O\&M Costs

Table 11 provides capital, operation, and maintenance (O\&M) cost, and efficiency for various flywheel systems.

Additional details on capital and O\&M costs are provided in Appendix 24 (Section 9).

\subsubsection{Pumped hydro power plant}

"This technology is the most developed and practiced utility storage option. The major difficulties are siting and environmental impacts of the two requisite reservoirs. As a possible feasible alternative for the pump hydro storage, a conventional hydro power plant could provide regulation service addressed by this project. The practical use of hydro power resources for wide-area regulation most likely depends on the availability of one of the existing hydro power plants for this service" (Makarov et al. 2008c).

\footnotetext{
122 Telephone conversation between M. Lazarewicz of Beacon Power with Viswanathanon September 12, 2011.

123 Telephone conversation between Lew Sibley of TSI and Viswanathan on September 26, 2011.

124 Telephone conversation between Bill Gray of Velkess Inc. with Viswanathan on September 27, 2011.
} 
"Pumped hydro power plant can provide both power regulation and energy storage services. Examples can be found in California, where some pumped storage power plants provide both AGC regulation and intraday energy storage capabilities. In the regulation mode, pumped storage units are following an AGC signal by changing their megawatt output around the preferred operating points. In this mode, the plants may be capable of providing the maximum ramp rate almost equal to their full capacity in one minute. This would be a sufficiently fast response for the purposes of this project. In this respect, the pumped energy storage can be used similarly to the use of conventional hydro power plants for regulation. A transition from the pumping to generation mode takes minutes, and, of course, this would not be an acceptable response time for the regulation purposes. The energy storage mode could be used to provide intraday services for the wind generation projects and to help in following the schedules, optimizing the daily production schedules, and addressing the overgeneration problem" (Makarov et al. 2008c). 
Table 11 Summary of capital cost diversity for flywheel systems

\begin{tabular}{|c|c|c|c|c|c|}
\hline $\begin{array}{l}\text { Capital cost } \\
(\$ / \mathrm{kW})\end{array}$ & $\begin{array}{l}\text { Capital } \\
\text { cost } \\
(\$ / k W h)\end{array}$ & $\begin{array}{l}\text { O\&M fixed } \\
\text { (\$/kW- } \\
\text { year) }\end{array}$ & $\begin{array}{l}\text { O\&M } \\
\text { Variable } \\
\text { cents/kWh }\end{array}$ & $\begin{array}{l}\text { Efficiency } \\
(\%)\end{array}$ & References \\
\hline $200-500(5 s)$ & 7 & $\begin{array}{l}2 \% \text { of } \\
\text { capital } \\
\text { costs }\end{array}$ & - & - & Taylor et al. 1999 \\
\hline 800 (UPS) & - & - & - & - & Taylor et al. 1999, \\
\hline $\begin{array}{l}200-500 \text { (few } \\
\text { min) }\end{array}$ & - & - & - & - & $\begin{array}{l}\text { Prodromidis et al. } \\
2011 \text {, }\end{array}$ \\
\hline $1000-3000(1 \mathrm{~h})$ & - & - & - & - & $\begin{array}{l}\text { Prodromidis et al. } \\
2011 \text {, }\end{array}$ \\
\hline $\begin{array}{l}1000(\text { Gen } 4 \\
\text { Beacon)(a) }\end{array}$ & - & - & - & $85 \%$ & Footnote ${ }^{125}$ \\
\hline 1630 & - & - & - & - & Rounds et al. 2008 \\
\hline- & $22(b)$ & - & - & - & $\begin{array}{l}\text { Bolund et al. } 2007 \\
\text { Liu et al. } 2007\end{array}$ \\
\hline- & $38(c)$ & - & - & - & Liu et al.2007 \\
\hline- & 104-290(d) & - & - & - & Bolund et al.2007 \\
\hline- & $165-250(e)$ & - & - & $95-97 \%(f)$ & $\begin{array}{l}\text { Footnote }^{126} \\
\text { footnote }^{127}\end{array}$ \\
\hline $200(\mathrm{~g})$ & 100 & - & - & $90-95 \%(h)$ & Footnote ${ }^{128}$ \\
\hline $\begin{array}{l}650(1 \mathrm{MW} / 0.25 \\
\mathrm{MWh})\end{array}$ & - & $20-30$ & - & - & $\begin{array}{l}\text { Walawalkar et al. } \\
2006\end{array}$ \\
\hline
\end{tabular}

(a) Excludes installation cost of $\$ 5 \mathrm{M}$ for a $20-\mathrm{MW}$ plant. Cost for Gen 3 was $\$ 2,000 / \mathrm{kW}$.

(b) Steel rotor.

(c) Graphite rotor.

(d) $\$ 104 / \mathrm{kWh}$ for Toray Carbon Fiber CT1000 from Toray and $\$ 290 / \mathrm{kWh}$ for HexTow® AS4C carbon fiber from Hexcel Corporation.

(e) $\$ 250 / \mathrm{kWh}$ for a $250-\mathrm{kWh}$ system and $\$ 165 / \mathrm{kWh}$ for MWh-sized system. Operation time $>1 \mathrm{~h}$.

(f) May not include PCS losses.

(g) Unit power (\$/kW) and energy (\$/kWh) costs provided separately for Tribology Systems, Inc. (TSI) systems.

(h) For MW/MWh high-voltage system. For 48 V DC system, efficiency is $85 \%$. May not include PCS losses.

\subsubsection{Superconducting magnetic energy storage (SMES)}

"In a SMES, the energy is stored in the magnetic field of a superconducting coil. The coil must be kept at a very low temperature to maintain its superconducting capability. Advantages include an extremely short response time, as well as a high efficiency (the superconducting coil itself is theoretically lossless, but the conversion from AC to DC and back implies losses, as does the continuous cooling of the coil)" (Makarov et al. 2008c).

"Applications of SMES are able to provide high power, very fast, but usually for very short periods (seconds). It has scaling potential to about 1-MWh capacity without serious siting

\footnotetext{
125 Telephone conversation between M. Lazarewicz of Beacon Power with Viswanathanon September 12, 2011.

126 Telephone conversation between Lew Sibley of TSI and Viswanathan on September 26, 2011.

127 Email received from Lew Sibley of TSI on October 27, 2011, by Viswanathan.

128 Telephone conversation between Bill Gray of Velkess Inc. with Viswanathan on September 27, 2011.
} 
restrictions, but the exposure of the surroundings to the magnetic field must be considered. Besides, the immaturity of the large-scale SMES systems capable of bulk storage is a major disadvantage" (Makarov et al. 2008c).

\subsubsection{Compressed air energy storage (CAES)}

"This has been an established, energy storage technology in grid operation since the late 1970s. With this technology, energy is stored mechanically by compressing air" (Makarov et al. 2008c).

\subsubsection{Physics}

"CAES uses electricity to compress air using an electric motor and store it either in underground caverns or in above-ground vessels or pipes" (Rastler 2010, Cavallo 2007).

"Underground storage comprises various geologic formations, such as salt caverns, rock mines, depleted oil and gas reservoirs, and aquifers; and it is suitable for hundreds of megawatts of storage for several hours" (Ridge Energy Storage and Grid Services 2005).

"Generation of electricity is carried out by expansion of the compressed air through a series of turbine generators. Conventional or diabatic CAES burns natural gas in a combustion turbine, thus generating power. The exhaust from the combustion turbine is used to heat the compressed air, which is expanded through a high-pressure and low-pressure turbine" (Drury et al. 2011). "In a variation of this, the compressed air is heated with natural gas without generating electricity. There are variations on the CAES concept that include thermal energy storage, operation in the adiabatic mode or isothermal mode, with elimination of the combustion turbine; or direct coupling of the compressor with wind turbines to avoid conversion of mechanical energy from the turbines to electricity" (Grazzini and Millazzo 2008).

\subsubsection{Main advantages and disadvantages}

"If the heat that develops during compression is conserved, this mechanical process is theoretically 100-percent efficient. However, in large-scale systems, that is not likely to be the case. Furthermore, combined with the losses occurring during the conversion from electrical to mechanical energy and back, the round-trip efficiency is low" (Makarov et al. 2008c). "The overall efficiency for diabatic CAES is $\sim 0.5$, while it is anticipated that efficiency as high as 0.7 can be obtained for adiabatic/isothermal CAES" (Drury et al. 2011). One of its limitations is the need to wait for a few minutes prior to reversing direction from charge to discharge, or vice versa. Ramp rates in the range of 15 to 40 percent of rated power per minute have been obtained. "Other disadvantages include slow response and fewer environmentally acceptable siting opportunities" (Makarov et al. 2008c).

\subsubsection{Current Status}

There are two operating CAES plants in the world, a plant in Huntorf, Germany, commissioned in 1978, and a 110-MW/26 h plant in McIntosh, Alabama, commissioned in 1991. The Huntorf plant, designed by ABB, has a rated output of $290 \mathrm{MW}$ over three hours (Succar and Williams 2008), with an overall efficiency of 42 percent and a heat rate of 5,870 kJ/kWh lower heating value (LHV). The Mclntosh plant, designed by Dresser-Rand, recuperates the turbine exhaust heat, thus improving the overall efficiency to 53 percent, with a heat rate of $4,330 \mathrm{~kJ} / \mathrm{kWh} \mathrm{LHV}$ (BINE Informationsdienst 2007, Succar and Williams 2008). Dresser-Rand supplied all the 
rotating equipment for this plant, while Energy Storage and Power (ESPC) was responsible for engineering, design, manufacture, testing, and commissioning of the plant (Lucas and Miller 2010, Nakhamkin et al. 2010). Other proposed projects, such as the limestone mine in Norton, Ohio, (800 MW) and plans by Ridge Energy Storage \& Grid Services L.P. for several CAES installations in Texas, have been described in Grazzini and Millazzo (2008). These plants are termed first generation, with compressed air being heated by a high-pressure and low-pressure combustor, and the heated air passing through a high- and low-pressure expander. In the second generation CAES plant developed by ESPC, one third of the power is generated by a combustion turbine, which heats atmospheric pressure air. The exhaust from the CT is used to heat the stored compressed air, which is passed through an expander to generate power. Separate power trains are used for the compression and expansion sections to improve reliability at the expense of higher cost (Nakhamkin et al. 2010). Additional information on CAES performance and life are presented in Appendix 24 (Section 10), and information on capital and O\&M costs are given in Appendix 24 (Section 10.1).

\subsubsection{Plug-in hybrid electric vehicles (PHEV)}

Projections by Pike Research indicate that the U.S. and China may be the leaders by 2015 , with estimated HEV/PHEV/EV sales of 280,000 units. In order for this to come to fruition, Li-ion battery costs, including electronics, must come down to $\$ 500 / \mathrm{kWh}$ from the present $\$ 1000 / \mathrm{kWh}$.

"Plug-in hybrid electric vehicles are vehicles that are equipped with a significant-sized battery. Special electronic equipment could allow the grid to utilize this battery while the vehicle is parked. The advantage of this approach is that the battery resource otherwise left unused is used when needed by the electric utility. However, this is a strongly dispersed resource that requires the development of a complicated information infrastructure to control the battery charge/discharge process. Besides, the technology is still at its early stage, and it is not available for a commercial application" (Makarov et al. 2008c).

\subsection{Energy Storage Comparison}

As discussed earlier, applications and desired life guide the selection of energy storage technology and sizing. Some storage technologies are tailored for high-power applications, while others high energy. For a particular battery and capacitor technology, cell design is guided by specific application needs; with thinner electrodes and separators appropriate for high power and vice versa. Without getting to detailed, a high-level comparison of all battery technologies is given in Table 12, addressing energy density, efficiency, life, maturity, and cost. 
Table 12. Comparison of various energy storage technologies.

\begin{tabular}{|c|c|c|c|c|c|c|c|c|c|c|c|c|c|c|}
\hline Metric & $\begin{array}{l}\mathrm{Pb}- \\
\text { acid } \\
\text { high } \\
\text { energy }\end{array}$ & $\begin{array}{l}\mathrm{Ni-Cd} \\
\text { high } \\
\text { energy }\end{array}$ & $\mathrm{Ni}-\mathrm{MH}$ & $\begin{array}{l}\mathrm{Na}- \\
\mathrm{S}\end{array}$ & $\begin{array}{l}\text { Na- } \\
\text { metal } \\
\text { halid } \\
\text { e }\end{array}$ & Li-Ion & Li-air & $\begin{array}{l}Z \mathrm{Zn}- \\
\text { air }\end{array}$ & $\begin{array}{l}\mathrm{Zn-Br} \\
\text { flow } \\
\text { battery }\end{array}$ & $\begin{array}{l}\text { Vanadium } \\
\text { redox Flow } \\
\text { battery }\end{array}$ & $\begin{array}{l}\text { Regenerative } \\
\text { fuel cell }\end{array}$ & $\begin{array}{l}\text { Electro- } \\
\text { chemical } \\
\text { capacitors }\end{array}$ & $\begin{array}{l}\mathrm{C} / \mathrm{PbO} 2 \\
\text { hybrid } \\
\text { capacitor } \\
\mathrm{s}\end{array}$ & $\begin{array}{l}\text { Flywheel } \\
\mathrm{s}\end{array}$ \\
\hline Wh/kg & 35 & 40 & 60 & 90 & 90 & 100 & 500 & 300 & 200 & 30 & $200-500$ & 2.5 & 13 & 5 \\
\hline Wh/L & 50 & 60 & 90 & & & 170 & 500 & 300 & 200 & & & & 4000 & \\
\hline W/kg & 126 & 144 & 150 & 170 & 170 & 900 & 100 & 100 & 100 & & & 3000 & & \\
\hline W/L & 180 & 216 & 220 & & & 1530 & 100 & 100 & 100 & & & & & \\
\hline $\begin{array}{l}\text { Round-trip } \\
\text { eff 15-min }\end{array}$ & 60 & 65 & 75 & 75 & 75 & 80 & 40 & 60 & 70 & 80 & $25-40(1)$ & 90 & 80 & $90-95 \%$ \\
\hline $\begin{array}{l}\text { Round-trip } \\
\text { eff } 5 \mathrm{~h}\end{array}$ & 80 & 80 & 85 & 85 & 85 & 90 & 60 & 75 & 80 & 80 & $25-40$ & 95 & 85 & $\begin{array}{l}\text { Not } \\
\text { applicabl } \\
\mathrm{e}\end{array}$ \\
\hline $\begin{array}{l}\text { Calendar life } \\
25 \mathrm{C}\end{array}$ & 10 & 12 & 10 & 15 & 15 & 12 & & 10 & 10 & $\begin{array}{l}10 \text { year } \\
\text { (stack } \\
\text { replace) }\end{array}$ & $\begin{array}{l}10 \text { year (stack } \\
\text { replace) }\end{array}$ & 20 & 15 & 10 \\
\hline $\begin{array}{l}\text { Cycle life } \\
80 \% \text { DOD }\end{array}$ & 400 & 1350 & 3150 & $\begin{array}{l}315 \\
0\end{array}$ & 2000 & 2500 & $\begin{array}{l}\text { very } \\
\text { poor }\end{array}$ & $\begin{array}{l}\text { varie } \\
s \text { with } \\
\text { powe } \\
r\end{array}$ & 2750 & $>15000$ & $>10000$ & 100000 & 10000 & 125000 \\
\hline TRL & 7 & 7 & 7 & 7 & 6 & 6 & 2 & 3 & 4 & 6 & 3 & 5 & 5 & 7 \\
\hline MRL & 7 & 7 & 7 & 6 & 6 & 6 & 2 & 3 & 4 & 5 & 4 & 5 & 5 & 5 \\
\hline$\$ / \mathrm{kW}$ & 75 & 75 & 60 & $\begin{array}{l}150 \\
0\end{array}$ & 1500 & 110 & & & & 1100 & $7000-10000$ & 20 & 7 & 1280 \\
\hline$\$ / \mathrm{kWh}$ & 300 & 350 & 370 & 415 & 500 & 1000 & & & 325 & 215 & & 27000 & 2337 & 150 \\
\hline $\begin{array}{l}\text { High-energy } \\
\text { application }\end{array}$ & Yes & Yes & Yes & Yes & Yes & $\begin{array}{l}\text { Yes } \\
\text { (expe } \\
\text { nsive } \\
\text { ) }\end{array}$ & $\begin{array}{l}\text { Not } \\
\text { yet } \\
\text { ready }\end{array}$ & $\begin{array}{l}\text { Not } \\
\text { yet } \\
\text { ready }\end{array}$ & Yes & Yes & $\begin{array}{l}\text { Yes - } \\
\text { expensive }\end{array}$ & No & $\begin{array}{l}\text { Better } \\
\text { than } \\
\text { capaci- } \\
\text { tors, but } \\
\text { still poor } \\
\text { energy } \\
\text { density }\end{array}$ & No \\
\hline $\begin{array}{l}\text { High-power } \\
\text { application }\end{array}$ & Yes & Yes & Yes & $\begin{array}{l}\text { No } \\
\text { E/P } \\
\text { ratio } \\
=5- \\
7\end{array}$ & $\begin{array}{l}\text { Yes } \\
\text { (E/P } \\
\text { ratio } \\
\text { of } 1 \\
\text { avail } \\
\text { able) }\end{array}$ & Yes & $\begin{array}{l}\text { Not } \\
\text { yet } \\
\text { ready }\end{array}$ & $\begin{array}{l}\text { Not } \\
\text { yet } \\
\text { ready }\end{array}$ & Yes & Yes & $\begin{array}{l}\text { Yes - } \\
\text { expensive }\end{array}$ & Yes & Yes & Yes \\
\hline
\end{tabular}


Intentionally blank. 


\section{Considerations for Demand Response (NERC 2010a)}

"Advances in communications and controls technologies are expanding the ability of consumers to both respond to system operator directives and to respond to price signals. Demand response (DR) is not a single technology. Rather, DR is any technology that controls the rate of electricity consumption rather than the rate of generation. FERC defines the term DR to include "consumer actions that can change any part of the load profile of a utility or region, not just the period of peak usage" (FERC 2010a).

FERC goes on to recognize DR as including devices that can manage demand as needed to provide grid services such as regulation and reserves, and changing consumption for the "smart integration" of variable generation resources (FERC 2010a). There are numerous existing DR technologies. The report focuses on new DR technologies and on DR technologies that are particularly well suited to help integrate variable renewable generation (NERC 2010a).

"DR technologies that meet established performance criteria could provide power system balancing needs, including the integration of renewable generation. Different technologies will be successful for different applications in different locations, depending on the specific characteristics of the local loads, as indicated by the above example.

"NERC and the North American Energy Standards Board (NAESB) characterize the full range of DR options as shown in Figure 38. Demand response can be very fast, as with under frequency load shedding, very slow, as with efficiency improvements, or anywhere in between. Electric Reliability Council of Texas (ERCOT) currently obtains half of its responsive (spinning) reserves (1,150 MW of the 2,300 MW total) through a DR product called loads-acting-as-resources (LAARs). These customers have under-frequency relays set to $59.7 \mathrm{~Hz}$ so that they automatically trip offline during under-frequency events. During emergency conditions, these loads will also disconnect upon receiving instructions from ERCOT. ERCOT also has an emergency interruptible load service (EILS), a separate program of loads that will separate from the system during emergency conditions upon receiving instructions from ERCOT. NERC and NAESB characterize DR as either being "dispatchable resource" or "customer choice and control." Dispatchable resources give the power system operator either direct physical or administrative control of the load's power consumption. Customer choice and control response are based on the consumer's voluntary response to price signals. Both types of programs can be effective in obtaining reliable response from loads. 


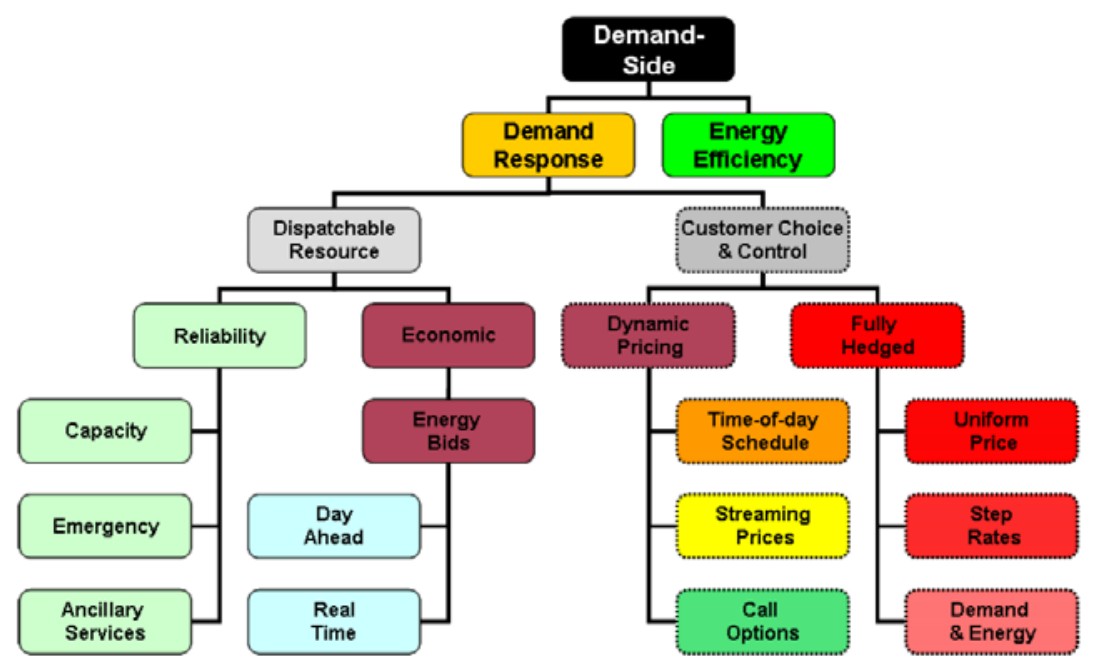

Figure 38. NERC and NAESB characterization of demand response (NERC 2010a). ${ }^{129}$

"Using DR as a capacity or energy resource in wholesale electricity markets is a relatively new concept, and grid operators are still working out how best to incorporate DR resources for ramping, balancing. and regulation. Organizations such as utilities, load-serving entities, grid operators, and independent, third-party, demand-response providers are developing ways to enable demand response to be used more broadly as a resource in energy, capacity, and ancillary services markets. New types and applications of DR are emerging due to technology innovations and policy directives. These advances have made it both technically feasible and economically reasonable for consumer response to signals from a utility system operator, loadserving entity, RTO/ISO, or other DR provider, to be deployed to provide reliability services to the bulk system.

"From the loads' perspective, in addition to the technical requirements of the reliability function to be provided (such as response speed, frequency, and duration), other important characteristics include sensitivity to electricity price and storage capability. Storage of intermediate product or energy at the load's premise is valuable in enabling the load to respond to power system needs, without hurting the loads' primary function.

"The majority of DR programs in use are designed to reduce peak demand. DR programs might also provide contingency reserves, as is the case in ERCOT. Most recently, a few loads have started to provide minute-to-minute regulation, providing an example of one extreme of DR capability. Air-conditioning loads (residential and commercial, central and distributed) can be ideal suppliers of spinning and non-spinning reserves. Many pumping loads are good candidates (water, natural gas, and other gasses). Any industrial process with some manufacturing flexibility is a good candidate (cement, paper, steel, aluminum, refining, air liquefaction, etc.).

"DR technologies not only respond to the signals of a load-serving entity or other curtailment service providers, but may also be designed to respond to conditions of the bulk power system, such as a change in system frequency. Competitive market forces enabled by the deployment of advanced metering infrastructure and dynamic pricing are expected to continue to support increased demand response and greater consumer control over energy use. Demand response-

\footnotetext{
${ }^{129}$ Reused by permission of NERC.
} 
based energy resources could support bulk system reliability as variable generation increases the need for certain reliability services. To fully realize the potential contributions from demand response, however, regulatory and institutional barriers need to be addressed" (NERC 2010a).

Additional information on the demand response is provided in Appendix 25.

\subsection{Capability/feasibility of providing system reliability functions (NERC 2010a)}

"DR encompasses such a broad range of technologies, select subsets of DR resources can be identified that are technically capable of providing each of the balancing functions required by the bulk power system. Some services are easier for loads to provide than others, and different loads are better suited to provide different responses. Characteristics of concern when evaluating DR technologies include:

- Response amount - MW

- Response speed - MW/minute

- Response duration - hours

- Response frequency - calls per day or week

- Recovery time - hours

- Response availability - based on time of day, season, ambient temperature, and other factors

- Required notification - immediate response versus day-ahead notification

"DR programs include dispatchable resource programs and customer-choice programs. Both types of demand response can provide a range of response capabilities with the dispatchable resources being required for the fastest regulation and contingency reserve response, while the customer-choice programs can provide hourly and sub-hourly response" (NERC 2010a).

\subsection{Inertial response (NERC 2010a)}

"Large motor loads provide natural inertial response, just like rotating generators. While individual loads tend to be smaller than individual generators, there are more loads. The natural inertial response of loads has been declining over the past several decades, for several reasons:

1. The percentage of large industrial motor loads has been declining relative to commercial and residential loads;

2. The proliferation of variable frequency drives on motor loads, which decouple the motor inertia from the power system frequency, removing the natural inertial response; and

3. The proliferation of higher-efficiency commercial and residential air conditioners that are both lighter (lower inertia) and increasingly controlled through variable-frequency drives.

While the natural inertial response of motor loads is declining, loads driven by solid-state power supplies could provide synthetic inertia by deliberately responding quickly to power system deviations. At this time, however, there is no requirement or incentive for loads to provide 
inertial response. As such, distributed resources contributions to system inertial response needs are not anticipated to be significant" (NERC 2010a).

\subsection{Primary frequency response}

"Similar to inertial response, loads driven by solid-state power supplies could provide primary frequency response if there was a requirement or an incentive. Responding to low frequency by reducing load typically is more useful for supporting power system reliability, and is inherently easier for most responsive loads than responding to high frequency by increasing load. Aggregations of individual loads also could provide smooth, primary frequency response through under-frequency load tripping of individual loads at different frequencies. The aggregation could provide the equivalent of a generator droop curve. Loads that sell spinning reserve or regulation might be required to also be frequency responsive. Generally, however, the potential contribution of DR to system primary frequency response needs is expected to be low" (NERC 2010a).

\subsection{Regulation}

"Regulation is typically the most difficult ancillary service for load to provide because of the communications and control requirements. System operators send AGC commands out every 2 to 8 seconds. The load must have the capability to move up and down rapidly and accurately. A few loads are beginning to provide regulation. To do so, the load must be able to respond to system operator AGC commands to increase or decrease consumption every few seconds. While regulation is a difficult service for loads to provide, advances in communications and control now make regulation response possible. The high price paid for regulation in hourly ancillary service markets provides a strong incentive for loads to develop regulation capability. While this economic incentive likely will result in more loads providing regulation services, a significant increase in penetration will be required for DR to contribute significantly to system regulation needs" (NERC 2010a).

\subsection{Load following/ramping}

"Some loads can adjust their energy consumption in response to system operator commands or to real-time energy prices providing sub-hourly to multi-hour energy balancing. Price-responsive load and peak shaving target specific hours when response is desired. Price-responsive loads facilitate a voluntary response to market price signals. Peak shaving uses direct control commands. Both price-responsive loads and peak shaving can be used to address capacity inadequacy caused by a lack of generation or a lack of transmission. Programs that have traditionally responded to periods of peak demand, summer hours for example, could be redesigned to respond to changes in variable generation. Simply allowing loads (residential, commercial, and/or industrial) to respond to real-time price signals will facilitate load following and ramping response. Based on the potential magnitude of load resource (which can participate in price responsive programs with relatively low economic barriers), it is anticipated that load may contribute moderately to future system load following/ramping needs. This will require, however, that the regulatory and/or institutional barriers discussed subsequently be adequately addressed" (NERC 2010a).

\subsection{Dispatchable energy (NERC 2010a)}

"Load can contribute to dispatchable energy needs through traditional peak reduction. Similar to load shifting, electricity consumers can adjust consumption to support the deliberate use of energy at times of high variable generation output, and low system-wide demand. When significant quantities of excess wind generation are forecast to be available, there may be an 
opportunity for certain loads to adjust their consumption to take advantage of the low-cost surplus. Space conditioning loads might heat or cool thermal storage at night and use the stored thermal energy the following day. Industrial loads may change their operations to use night-time energy. The ability to economically use the resource depends on a reliable, long-term forecast of the energy's availability. Loads that are designed to make use of the surplus could also be designed to be responsive to the variability of its supply. As with load following/ramping, the potential magnitude of load resource and relative low barriers to participation suggest that DR can have a moderate contribution to system dispatchable energy needs (minimum and peak load management)" (NERC 2010a).

\subsection{Contingency spinning reserve service (NERC 2010a)}

"Supplying spinning reserve is attractive for some loads since the response duration, and therefore the interruption in the load's normal course of business, is limited. Additionally, spinning reserve is called on relatively infrequently. Response speed is critical but not overly burdensome for some loads. Space conditioning from residential and commercial loads can be an attractive source for spinning reserve, as can numerous specific industrial processes. Based on the large, potential resource from space conditioning and industrial loads, and relatively low technical barriers to participation, it is anticipated that DR may make a significant contribution to system spinning reserve requirements" (NERC 2010a).

\subsection{Contingency non-spinning reserve (NERC 2010a)}

"Appropriately, responsive loads are typically allowed to supply non-spinning reserve. Supplying non-spinning reserve is attractive for the same reasons supplying spinning reserve is attractive: namely, that the required response is infrequent and limited in duration. Similarly, it is anticipated that DR may make a significant contribution to system non-spinning reserve requirements."

\subsection{Replacement or supplemental reserve (NERC 2010a)}

"Appropriately, responsive loads typically are allowed to supply replacement or supplemental reserve. With additional time allowed for response additional loads typically are able to provide supplemental reserves. As such, it is anticipated that DR may make a significant contribution to system non-spinning reserve requirements" (NERC 2010a).

\subsection{VG tail event reserve (NERC 2010a)}

"Loads that can supply replacement reserve or supplemental operating reserves typically will be able to supply variable generation tail event reserves. Variable generation tail event response is slow enough that market response might be effective. Sufficient time is available to allow an aggregator to obtain the required response from a fleet of individual loads with diverse characteristics. As such, it is anticipated that DR may make a significant contribution to system non-spinning reserve requirements" (NERC 2010a).

\subsection{Voltage support (NERC 2010a)}

"DR is not well suited for supporting system voltage or reactive needs. Reducing loads may have a small, localized impact on system voltage, but the ability to supply reactive power to support bulk system voltages is limited. It is possible that future scenarios, where residential and commercial loads include distributed solar photovoltaic generation, the inverters from the PV might be aggregately dispatched to support bulk system voltage, but this concept is only 
beginning to be considered. As such, it is anticipated that DR will have a low contribution to system voltage support needs" (NERC 2010a).

\subsection{Wide-scale deployment and associated risks (NERC 2010a)}

“FERC's 2009 National Assessment of DR Potential provides a national estimate of its technical potential in 5- and 10-year horizons. Under the full-participation scenario (the most aggressive scenario, which assumes national deployment of advanced metering infrastructure and dynamic pricing), FERC's assessment finds the DR potential to be $188 \mathrm{GW}$ by 2019 . Under the businessas-usual scenario, which represents a continuation of existing best practices, FERC estimates that 37 GW of DR would be achieved by 2019" (FERC 2009).

"Given this potential resource magnitude, and the technical capabilities summarized in the previous section, DR could provide a significant fraction of several of the reliability functions required by the power system. There are, however, potential risks to this level of DR deployment, and use for supplying reliability functions and services. Institutional, rather than technical barriers are the most significant obstacles to greater use of DR. Loads are prevented from providing response in many areas. While ERCOT currently obtains half of its contingency reserve from responsive load, ERCOT limits the portion of required contingency reserves that can be served by loads to half, as current market rules do not allow ERCOT to obtain response at staggered frequency levels. WECC does not allow loads to provide spinning reserve. Many state regulators do not allow retail loads to be exposed to real-time prices, effectively blocking balancing energy response. Even jurisdictions that do allow such exposure (e.g., New York and New Jersey) have seen relatively limited customer acceptance of real-time pricing (RTP). Some ISOs co-optimize energy and ancillary service procurement, an excellent practice that helps both generators and the power system maximize efficiency. Unfortunately, it can turn a regulation or contingency reserve offer into a requirement to supply energy for hours. This effectively blocks both storage and responsive loads from offering regulation or contingency reserves. This is beginning to be addressed for storage, but it is still an effective barrier to DR for reliability services. It is difficult to predict when or if the institutional barriers that currently limit DR will be addressed. Realization of DR is providing reliability. Potential reliability impacts of emerging flexible resources functions to the grid on a large scale will require state regulators, utility managers, and other stakeholders to collectively address non-technical, institutional, and/or regulatory barriers to take advantage of these resources" (NERC 2010a).

"In addition to the institutional and regulatory barriers, additional technical developments are also needed to facilitate large-scale deployment of DR for providing some of the specified reliability functions. Communications and control requirements are critical elements when determining whether load is capable of providing reliability reserves. Load must be controllable and dispatchable if it is to supply reliability services to the power system as would a generation resource. The controls must be fast and accurate, with capabilities to communicate and receive commands from the power system operator. Faster services (spinning reserve and regulation) require an automatic response to system operator commands and, in some instances, changes in power system frequency. The required response speed and duration depend on the reliability service being provided" (NERC 2010a).

"Aggregation of smaller loads and DR participants is an important component for the broad use of DR. It is the combined response of the collection of responding loads that is important for power system reliability, rather than the response of an individual load. An aggregator may be able to manage a fleet of loads to provide response that is more certain and predictable than can be provided by any of the individuals. Again, however, this will require the development of a 
suitable communication and control infrastructure to support an aggregator's ability to use the collective resources" (NERC 2010a).

\subsection{Distribution interconnection - technical concerns (Kroposki 2009)}

\subsubsection{Short-circuit current coordination (Kroposki 2009)}

"DR can provide a variety of short-circuit current levels. There has to be a sufficient level of short-circuit current available within the separated, local electric power system (EPS) and distributed energy resource (DER) system to clear short-circuit faults. The separated, local EPS and DR system has to provide enough fault current to operate the protective devices in the system - including circuit breakers, fuses, and fault protection relays. See Figure 39" (Kroposki 2009).

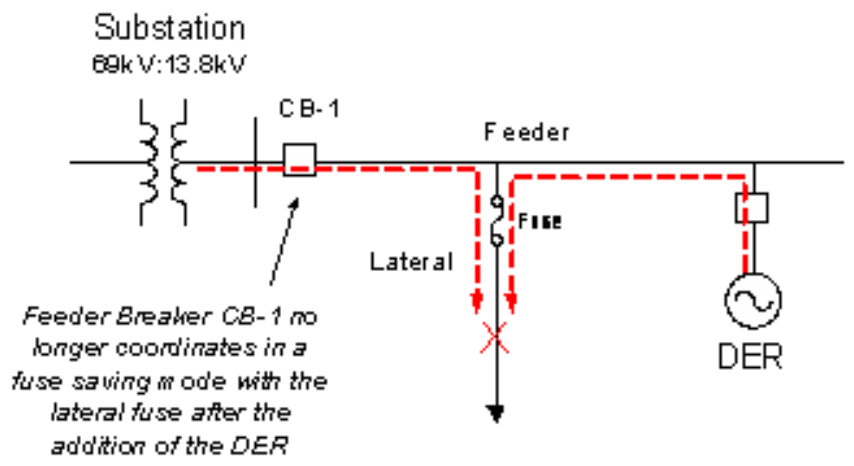

Figure 39. Short-circuit current coordination (Kroposki 2009). ${ }^{130}$

\subsubsection{Recloser coordination (Kroposki 2009)}

"Automatic circuit reclosers also may be deployed in the feeder circuit to clear faults and quickly restore service on the feeder. Reclosers reenergize the circuit automatically immediately after a trip resulting from a feeder fault. The response of the DR unit must be coordinated with the reclosing strategy of the isolations within the area EPS. Coordination is required to prevent possible damage to area EPS equipment and to equipment connected to the area EPS other than the DR. See Figure 40" (Kroposki 2009).

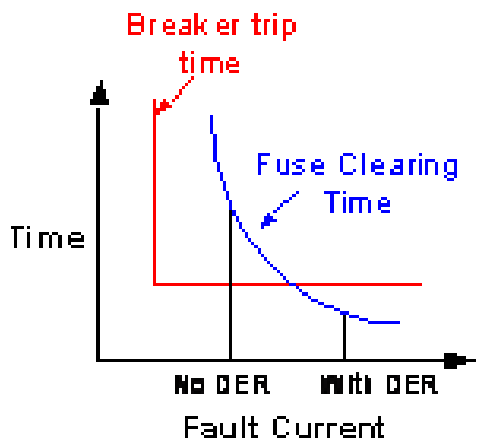

Figure 40. Recloser coordination (Kroposki 2009). ${ }^{131}$

\footnotetext{
${ }^{130}$ Reused by permission of NREL.

${ }^{131}$ Reused by permission of NREL.
} 


\subsubsection{Voltage regulation (Kroposki 2009)}

"The primary objective of voltage regulation is to provide each customer connected to the utility with voltage that conforms to the design limitations of that customer's utilization equipment.

Voltage supplied to each customer at the Point of Common Coupling (PCC) is an important measure of service quality. Satisfactory voltage level is required to operate lights, equipment, and appliances properly.

Injecting power from a DR device into the power system will offset load current, thus reducing the voltage drop on the utility. The DR device may inject leading reactive power (capacitive) into the power system, or draw lagging reactive power (inductive) from the power. See Figure 41" (Kroposki 2009).

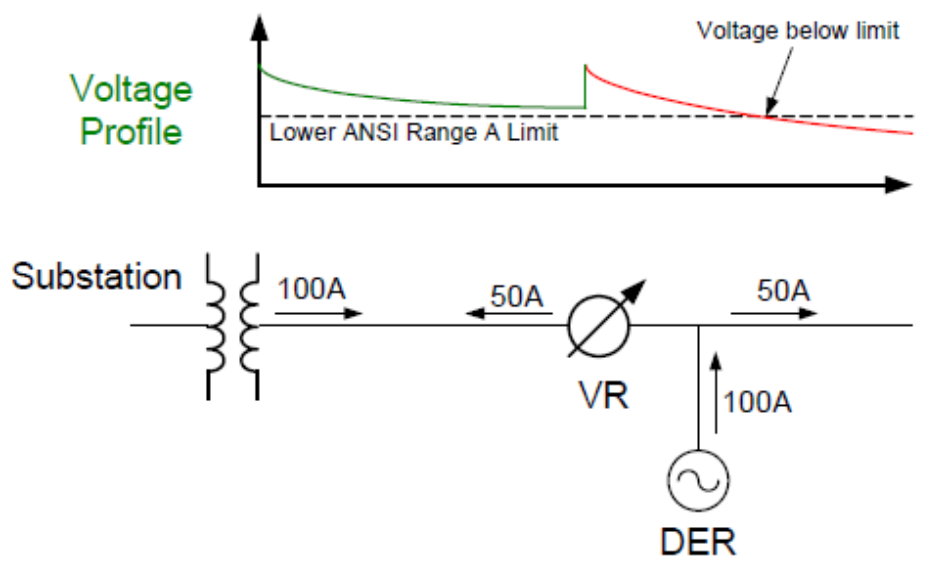

Figure 41. Voltage regulation (Kroposki 2009). ${ }^{132}$

\subsubsection{Grounding (Kroposki 2009)}

"Depending on how the DR is constructed within the local EPS, there could be potential grounding conflicts set up in the installation. If the point of interconnection for the DR occurs within a building inside a service entrance, then this issue is more likely to be a concern. Under normal conditions, the DR generator is a grounded source because it receives a ground reference from the grounding of the electrical service at the building's service entrance, as required by building codes. Those codes also require that the service is grounded only once at the building's service entrance, and thus, a local grounding of the DR generator elsewhere in the building premises would be prohibited. Then when the abnormal conditions do occur, and the DR separates from the grid and the grounded service, suddenly the DR generator loses its independent ground reference" (Kroposki 2009).

\subsubsection{Power quality issues (Kroposki 2009)}

This section addresses electrical power quality issues.

\footnotetext{
${ }^{132}$ Reused by permission of NREL.
} 


\subsubsection{Harmonics (Kroposki 2009)}

"Harmonic currents cause transformers to overheat, in turn, overheating neutral conductors. This overheating may cause erroneous circuit breaker tripping and other equipment malfunctions. The voltage distortion created by nonlinear loads may create voltage distortion beyond the premise's wiring system, through the utility, to another user. DR should be designed to inject a minimal amount of harmonic distortion onto the grid. See Figure 42" (Kroposki 2009).

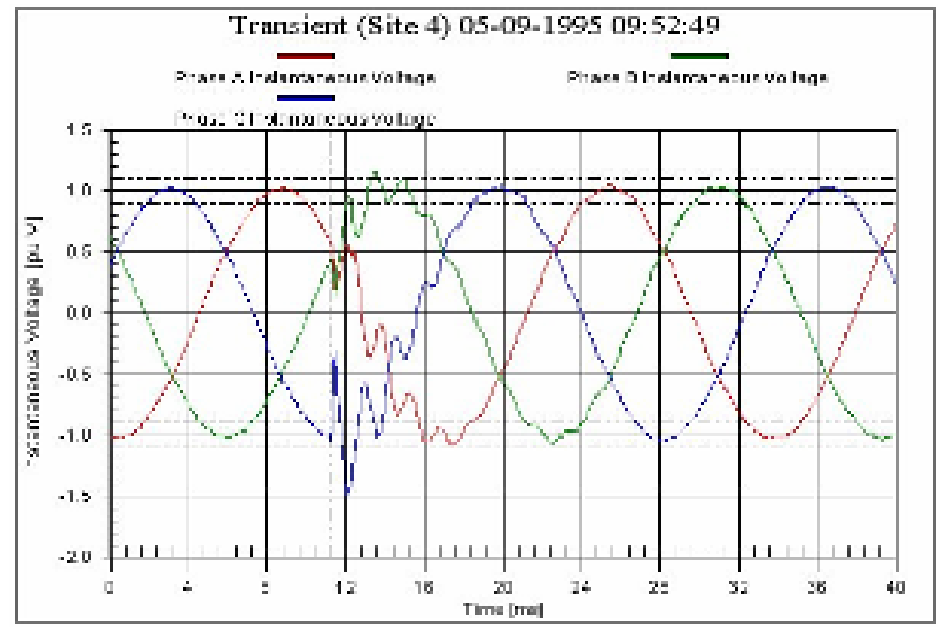

Figure 42. Harmonics (Kroposki 2009). ${ }^{133}$

\subsubsection{DC injection (Kroposki 2009)}

"DC injection produces a DC offset in the basic power system waveform. This offset increases the peak voltage of one-half of the power system waveform (and decreases the peak voltage in the other half of the waveform). The increased half-cycle voltage has the potential to increase saturation of magnetic components, such as cores of distribution transformers. DR should be designed to not inject DC current onto the grid" (Kroposki 2009).

\subsubsection{Flicker (Kroposki 2009, Wind 2006)}

"Flicker is a power-quality issue that is predominately associated with noticeable changes in incandescent light output, which is caused by minor changes in voltage levels. Flicker can exist in fluorescent lighting, but it requires somewhat larger voltage deviations. DR should not cause flicker" (Kroposki 2009).

"During startup and generator switching, there will be inrush currents, which will cause the voltage to dip or flicker. Voltage flickers may or may not be noticeable or objectionable. This depends upon magnitude and how often it occurs... The magnitude of flicker depends on the stiffness of the line, voltage level, distance from substation, size of substation transformer, and wind turbine electrical design" (Wind 2006).

\subsubsection{Intentional and unintentional islanding (Kroposki 2009)}

"Unintentional islanding occurs when the distributed generator (or group of distributed generators) continues to energize a portion of the power system that has been separated from

\footnotetext{
${ }^{133}$ Reused by permission of NREL.
} 
the rest of the utility. In most cases, it is not desirable for a DG to island with any part of the utility on an unplanned basis; this can lead to safety and power quality problems that will affect the area EPS and local loads. During utility repair operations, such as dealing with downed conductors, DG islanding can expose utility workers to circuits that otherwise would be deenergized (and the workers believe to be de-energized). IEEE 1547 requires that DR interconnection systems shall detect the island and cease to energize the area EPS within two seconds of the formation of an island.

Intentional islanding can be used to improve customer reliability, improve power quality, and can be used by utilities for maintenance" (Kroposki 2009). 


\section{Other Considerations Influencing Grid Planning}

This section addresses consolidation and cooperation among Balancing Authorities (BAs) and microgrids.

\subsection{Consolidation and cooperation among BAs (Makarov et al. 2010a)}

Because of the variability and uncertainty of wind and solar generation, the high penetration of renewable generation makes it more difficult for BAs to maintain balance between their load, interchange, and generation. Wide-area consolidation could significantly reduce production cost. Operating separately and locally, individual BAs would have to purchase more expensive balancing reserves to accommodate the variability and uncertainty from future high penetration of VG. Cooperation and consolidation between BAs has been identified as one of the most important strategies to facilitate high-level VG penetration while limiting requirements for generation reserves.

Consolidation of BA processes already takes place in the industry. For example, the consolidation of 26 BAs in the Midwest Independent System Operator (ISO) area into a single BA took place on January 6, 2009. In 2006-2007, four BAs ${ }^{134}$ formed a new cooperation by combining their Area Control Error (ACE) in the Pacific Northwest. Later, the ACE Diversity Initiative (ADI) was extended over a larger geographical region in the Western Interconnection, by including eight more BAs. ${ }^{135}$ Similar processes take place elsewhere in the world. In 2005, two Danish Transmission System Operators ${ }^{136}$ (TSOs) merged, forming a consolidated TSO called Energinet.dk. All wind power production and its deviations in Germany are combined virtually, and then are distributed to each of four transmission system operators. ${ }^{137}$

\subsubsection{Challenges facing individual BAs at high VG penetration levels}

In an electric power grid, generation and load must remain balanced all the time. Significant imbalances could result in significant interconnection frequency deviations, transmission system violations, stability problems, and so on. Ultimately, these problems could cause widespread system blackouts. The load varies with time and is (to a large extent) not controllable.

Generation must be dispatched to follow the load variation to ensure an adequate balance of supply and demand. To accommodate the variability and uncertainty of the load, some generation must be reserved for reliable grid operation.

High penetration of VG increases the challenges of maintaining power system balance. VGs are not yet dispatchable resources in the traditional manner. Wind power can only be produced when the wind blows. Likewise, solar power can only be produced when the sun shines. Wind and solar generators usually supply "must take" energy for BAs, which have little to no control over their power output. Often, the power generation from these resources is treated as negative load, so that the remaining generators are committed and dispatched to balance against so-called "net load," which is the difference between the BA's load, the interchange, and

\footnotetext{
${ }^{134}$ British Columbia Transmission Corporation, Idaho Power, Pacificorp East, Pacificorp West, and Northwest Energy.

135 Arizona Public Service, Nevada Power/Sierra Pacific Power, Public Service Company of New Mexico, Salt River Project, Seattle City Light, Bonneville Power Administration (BPA), Public Service Colorado/Xcel Energy, and Glacier Wind Farm (Naturener).

${ }^{136}$ Eltra and Elkraft.

${ }^{137}$ Amprion GmbH, 50Hetz Transmission Europe GmbH, Transpower Stromuebertragungs GmbH and EnBW Transportnetze AG.
} 
the VG. Renewable power resources increase the power variability and uncertainty, which has to be balanced. Specific problems with system reliability and control performance, which may occur because of the increasing VG, include:

- Increasing risk of system imbalances potentially harming BA control performance;

- Overgeneration;

- Fast unpredicted ramps;

- Unpredicted transmission overloads and loop flows;

- Voltage deviations;

- Decreasing system frequency response and inertia:

- Stability issues; and

- Adverse impact on conventional generators (including increasing cycling, wear-and-tear and emissions and decreasing efficiency).

The challenges of maintaining load-generation balance become more significant when each individual BA operates an interconnected power grid locally and separately. In North America, power grids are divided into many balancing areas to allow autonomy in operation. For example, the U.S. Western Interconnection is a large, integrated and interconnected power system. Organizationally, it is divided into 37 BAs. Within each BA, operators are responsible for maintaining the balance between load and generation within their territory, as well as for following interchange schedules between BAs. Metrics (e.g., area control errors and control performance standards) are used to evaluate the performance of each BA in maintaining the balance. BAs that do not meet the balancing requirements could jeopardize system reliability and are thus penalized financially. It has been proven that the impact of intermittency and uncertainty caused by VGs becomes relatively smaller if these resources are lumped together over a large geographical area, and if they are more dispersed over a territory. The existing BA structure is limiting the potential of wide-area integration of VGs because the BAs have to deal with their own local VG individually. In addition, each BA must maintain balance with its own, sometimes limited, resources. A BA with limited balancing reserves, and high wind and solar power penetration, will face significant problems. To maintain the balance, it frequently may have to resort to more-expensive resources. Sometimes, it may even run out of resources to maintain balance. Associated economic and reliability concerns may create hurdles to high-level penetration of VG under the existing BA structure and operational practices.

Some additional problems associated with the operation of individual BAs are:

- Control area operators do not have a broad view of the system.

- Individual BAs do not have the authority or mechanisms to achieve maximum efficiency on a system-wide basis.

- Independent decisions by individual BAs may generate negative impacts on other control areas.

- Transmission system flexibility becomes increasingly insufficient to accommodate all variability of power transfers.

- There is an increasing difficulty managing unscheduled flows on the transmission system, leading to reliability risks. 


\subsubsection{Motivations for BA cooperation}

More VG can be integrated into the current power grid through the cooperation of BAs. BA cooperation is, perhaps, the least-expensive option among the other possible solutions toward achieving RPS objectives. A recent General Electric Energy (GE) study, conducted for NREL, states explicitly that to integrate 35 percent of wind and solar resources in the area served by the WestConnect group of utilities, and 25 percent of these resources in the rest of the WECC system, BA cooperation and consolidation is required, among the other measures. When BAs cooperate with each other, the total variability will be less because of the geographical diversity. Wind and solar generation, as well as the associated forecasts errors, are generally not strongly correlated with each other over a wide geographic area. Thus, the combined, large-area power variation is smaller than the sum of variations for individual BAs. Such variability reduction can be shared among the participating BAs. Problems with balancing the system can be also mitigated through BA collaboration. The balancing generation resources in participating areas can be shared and more effectively used to manage power variations through coordination. Working together, BAs can balance reduced power variance with shared resources. This approach benefits power system operation from both economic and reliability perspectives. In addition, with the rapid development of information technology in the power industry, cooperation among individual BAs is becoming more viable.

\subsubsection{Benefits from BA cooperation}

Numerous benefits from the cooperation of individual BAs can be identified and evaluated:

- Reduced production cost - illustrates the expected reduction in production cost.

- Reducing regulation requirements in terms of its required upward and downward reserved capacity, actual use (energy), reduced impact on the regulation units (wear and tear, efficiency), and cost.

- Reducing load following requirements in terms of its required upward and downward capacity, actual use (energy).

- Reducing ramping requirements. Fast and continuous ramps, especially unexpected, create problems for grid operators. The ramping capability of most thermal generators is limited, so to follow fast ramps, more generators need to be used, which means more online capacity.

- Improving BA performance (control performance standard (CPS1/2) indices).

The benefits of BA cooperation and consolidation increase when renewable generation penetration increases.

\subsubsection{Major BA cooperation strategies}

BAs have been developing various measures to mitigate VG integration problems, including:

- Improved day-ahead, hour-ahead and real-time wind and solar forecasting systems (and wind ramp forecasting systems);

- Incorporating these forecasts into power markets, scheduling and real-time processes;

- Increasing operating reserve requirements;

- Grid codes requiring at least partial dispatchability from wind generators; 
- Additional energy storage;

- Demand response programs;

- New regional markets for the intra-hour balancing services;

- Coordinated, wide-area, real-time congestion management;

- Building special control centers for wind;

- Transmission system enhancements;

- Adding more flexible generation to the system;

- Increasing operating reserves (especially, intra-hour balancing reserves); and

- Dynamic scheduling and pseudo-tie options to deliver more ancillary services from the neighboring BAs.

Many of these methods are expensive options. BA cooperation and consolidation options are perhaps, among the least-expensive measures to mitigate the impacts of wind and solar power variability, poor predictability, and uncertainty.

BA cooperation can be implemented using different strategies. Each strategy has its own advantages and disadvantages. There is a minimum set of requirements that needs to be adopted by BAs to have successful cooperation. The objective of such strategies is to reduce the overall variability and uncertainty of the net load so that individual BAs within a large power grid can accommodate more VG without a significant increase of generation reserves. The major cooperation strategies are briefly described below.

\subsubsection{BA consolidation}

The consolidation of individual BAs is the integration of two or more BAs into a single Consolidated Balancing Authority (CBA). There are multiple ways and cooperation levels that can be used to consolidate BAs, e.g., full actual consolidation and virtual or partial consolidation.

The full, actual consolidation implies the actual merging of BAs into a single CBA. Under this option, the former BAs stop scheduling their individual interchanges except for the one between the common CBA and the rest of interconnection, they introduce common unit commitment and dispatch procedures, procure common operating reserves, use common automatic generation control (AGC), and so on. The full, actual consolidation helps to exploit the maximum benefits of consolidation.

The virtual or partial consolidation option means that the existing BAs and their certain functions are preserved in the CBA, and remain separated from the corresponding other BAs' functions. For instance, participating BAs may select to schedule and dispatch their own generators and provide intra-day and intra-hour balancing functions individually, based on sharing certain reduced, collective CBA requirements. For the partial consolidation option, it is important to underline which features of the consolidated BA are important to fully exploit potential benefits of consolidated operation.

\subsubsection{Area control error (ACE) diversity interchange (ADI)}

The laws of physics require that a balance between load and generation be achieved at the whole power grid level (unless it causes transmission system violations). But the reality is that, working separately, each BA needs to achieve local balance within its territory. Area control error is used to check how well a BA balances its generation against the native net load and interchange schedule. Automatic generation control systems are used to adjust special 
generation resources to achieve balance on a minute-to-minute basis. There are times when a BA instructs its AGC-connected generators to produce more power than the original schedule, while another BA in the same grid instructs its AGC-connected generators to reduce their output. When the excessive power generated in one BA is equal to the excessive power consumed in another BA, the whole power grid is well balanced. But, if there is no cooperation between BAs, each BA still has to adjust its regulation generation to achieve local balance.

Regulation reserve is an expensive resource. The main idea of ADI is to let all participating BAs pool excessive generation and consumption together to net out some collective adjustment requirement. The goal is to reduce the regulation required to balance the system. Relaxed control can be achieved because of the ACE diversity.

\subsubsection{Wind-only $B A$}

Unlike a traditional BA - which includes generating units and load - a wind-only BA includes only VG resources. Wind-only BAs do not have to stay in one concentrated location. VGs, which are distributed over a larger geographical region, can still be put together to form a wind-only BA to benefit from the geographical diversity factor. The wind-only BA is responsible for controlling the interchange of its control area with the other BAs following a predetermined interchange schedule. BAs' control performance is mandated by North American Electric Reliability Corporation and Western Electricity Coordinating Council Control Performance Standards. Generators participating in the wind-only BA will be required to provide their own balancing reserves or purchase those services from external resources. One of the operating wind-only BAs is Glacier wind energy (Mizumori, M. and B. Nickell. 2008).

\subsubsection{Dynamic scheduling}

Through telemetry, dynamic scheduling is a service that allows load or generation to be virtually transferred from one BA to another BA. Dynamic scheduling allows the receiving BA to control the generation or load in the sending $B A$, as if the generation or load was physically located in that BA. Dynamic scheduling could be used to bring more external renewable resources into a control area to meet the RPS requirements, while exploiting the geographic diversity factor. On the other hand, dynamic schedules can be used to incorporate external balancing resources helping to deal with VG variability. A summary of various BA consolidation and cooperation options are provided in Appendix 26.

\subsection{Microgrids ${ }^{138}$}

Microgrids are "coordinated energy and electrical distribution systems capable of independent operation that includes power sources for critical facilities during outages. Renewables can be incorporated to extend the fuel supply of conventional generators and provide a perpetual, reliable source of energy. They provide remote locations with a more robust, cost-effective power supply" (Butt 2011). Figure 43 shows a microgrid schematic taken from Kroposki et al. (2008). "Microgrids are intentional islands formed at a facility or in an electrical distribution system that contain at least one distributed energy resource and associated loads. Microgrids that operate both electrical generation and loads in a coordinated manner can offer benefits to the customer and the local utility. The loads and energy sources in a microgrid can be disconnected from and reconnected to the utility system with minimal disruption, thereby improving reliability. Any time a microgrid is implemented in an electrical distribution system, it must be well planned to avoid problems" (Kroposki et al. 2008).

\footnotetext{
${ }^{138}$ Contributed by Yuri Makarov, PNNL.
} 


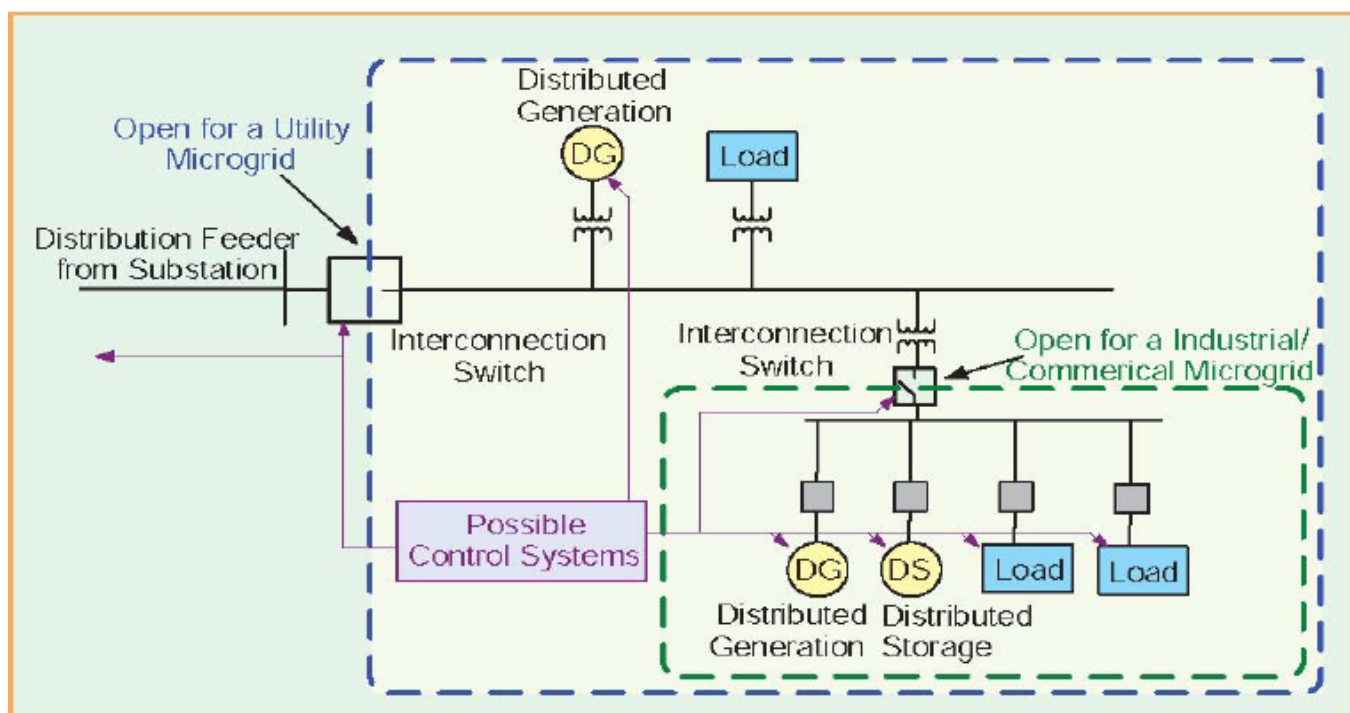

Figure 43. Microgrid structure (Kroposki et al. 2008). ${ }^{139}$

\subsubsection{Microgrid Standard IEEE 1547.4}

Microgrid standard EEE 1547.4 is a guide for design, operation, and integration of distributed resource island systems with electric power systems.

IEEE 1547.4 provides approaches and good practices for the design, operation, and integration of distributed energy resource (DER) island systems with electric power systems (EPS). This includes the ability to separate from and reconnect to part of the area EPS, while providing power to the islanded local EPSs. The IEEE standard includes the information on distributed resources, interconnection systems, and participating electric power systems. Because of some of the confusion surrounding the definition of microgrid in 2005 (and continues today), IEEE 1547.4 developed the term distributed resource island system, which is sometimes referred to as a microgrid. The term is used for electric power systems that:

1. Have DER and load;

2. Have the ability to disconnect from and parallel with the area EPS;

3. Include the local EPS and may include portions of the area EPS: and

4. Are intentionally planned.

DER island systems can be either local EPS islands or area EPS islands.

In IEEE 1574, an island is defined as a condition in which a portion of an area EPS is energized solely by one or more local EPSs through the associated PCCs while that portion of the area EPS is electrically separated from the rest of the area EPS. An intentional island is a planned island, and an unintentional island is an unplanned island. For an unintentional island in which the DER energizes a portion of the area EPS through the PCC, the DER interconnection system shall detect the island and cease to energize the area EPS within two seconds of the formation of an island. The intentional islanding is under consideration for future revisions of this standard. Figure 44 shows examples of distributed resources island systems (IEEE Standards Association. 2011).

\footnotetext{
${ }^{139}$ Reused by permission of IEEE.
} 


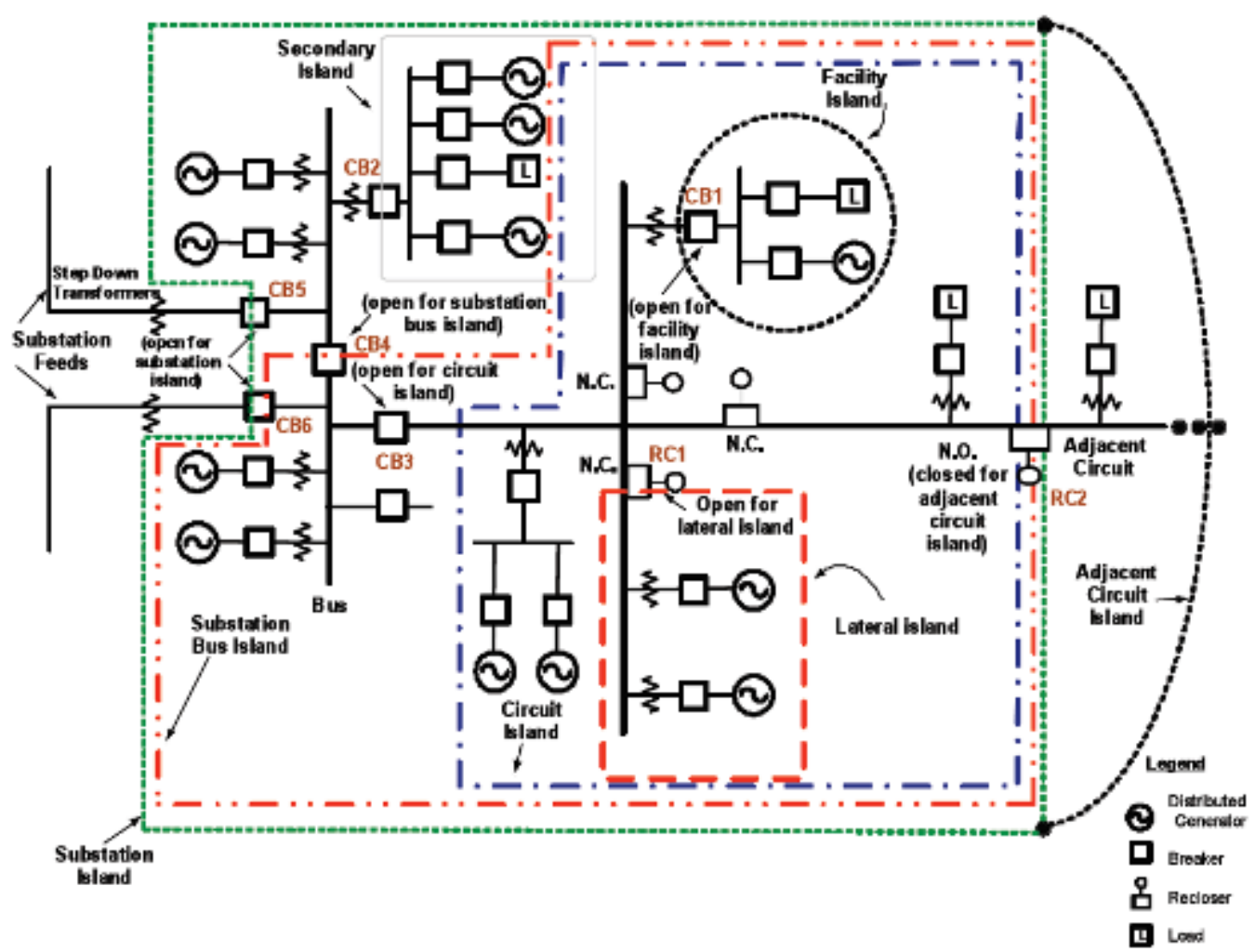

(1) IEEE 1547.4-2011

Figure 44. Examples of distributed resources island systems (IEEE Standards Association 2011). ${ }^{140}$

Some examples by which this requirement may be met are :

- The DER aggregate capacity is less than one-third of the minimum load of the local EPS.

- The DER is certified to pass an applicable, non-islanding test.

- The DER installation contains reverse or minimum power flow protection, sensed between the point of DER connection and the PCC, which will disconnect or isolate the DER, if power flow from the area EPS to the local EPS reverses or falls below a set threshold.

- The DR contains other non-islanding means such as:
a. forced frequency or voltage shifting;
b. transfer trip; or
c. governor and excitation controls that maintain constant power and constant power factor.

${ }^{140}$ Reused by permission of IEEE, 3 Park Avenue, New York, NY 10016-5997 USA, Copyright 2011, by IEEE. 


\subsubsection{Microgrid Planning and Design (Butt 2011)}

One of suggested sequences of microgrid design is shown in Figure 45.

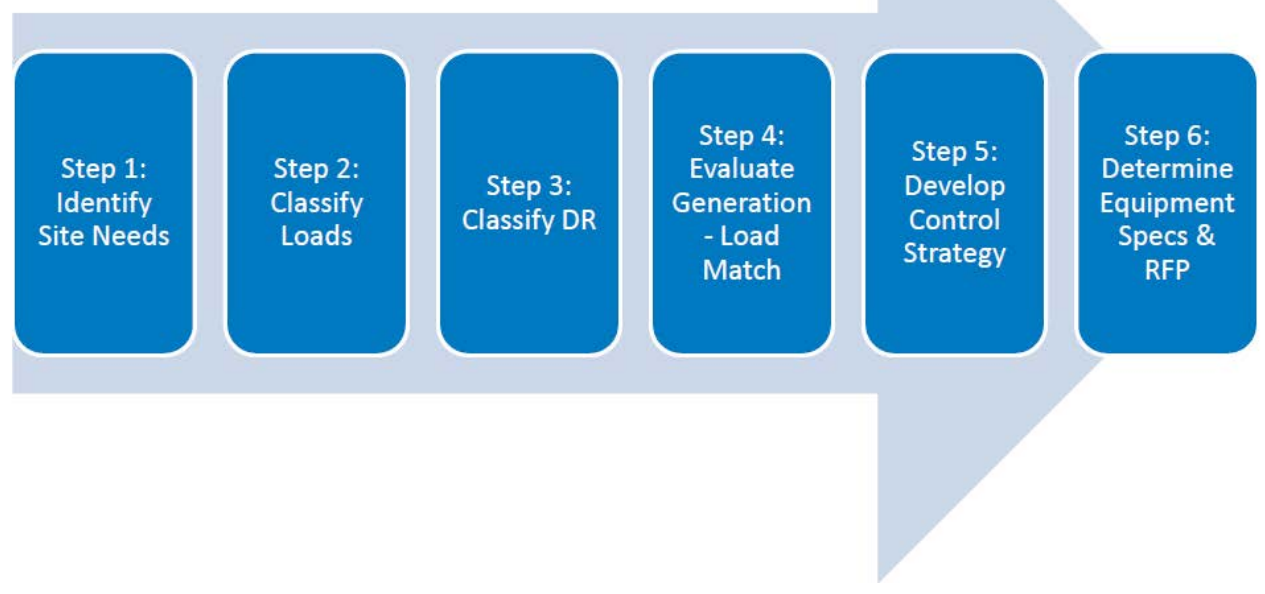

Figure 45. Microgrid planning and design (Butt 2011). ${ }^{141}$

10.2.2.1 Step 1: Identify site requirements (Butt 2011)

"Clarify the goals of the microgrid:

- What are the critical facilities that must be included in the microgrid?

- What are the thresholds and how long should a utility disturbance persist before transitioning to islanded mode?

- What is the maximum amount of time the microgrid must operate?

Factors impacting microgrid capabilities:

- Site mission;

- Geographic relationship of facilities and site electrical distribution system layout/characteristics;

- Existing standby generation capacity and controls;

- Availability and feasibility of renewable resources;

- Building management and control systems/load control schemes; and

- Utility standards and response to microgrid proposal" (Butt 2011).

${ }^{141}$ Reused by permission of NREL. 


\subsubsection{Step 2: Classify loads (Butt 2011)}

- “Load profiles - see Figure 46;

- Energy, annual and daily peaks, and seasonal behavior;

- Advanced Metering Infrastructure (AMI) data;

- Install temporary metering where necessary to record load characteristics;

- Operational and nameplate equipment data;

- Criticality of loads and sensitivity to power quality issues;

- Identify UPS (ride-through) locations; and

- Flexibility of load to adjust and match available generation" (Butt 2011).

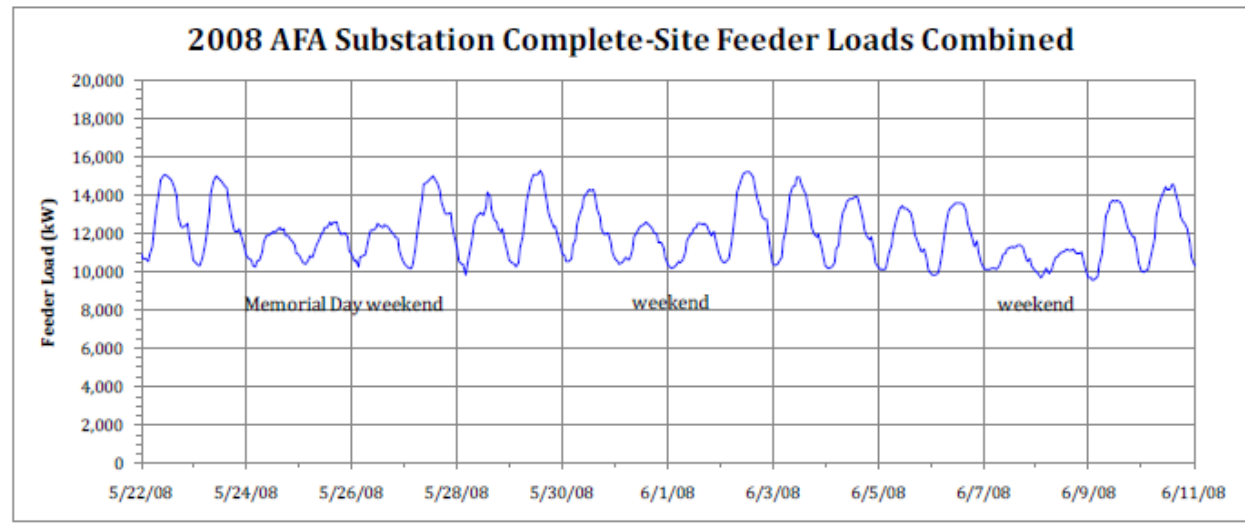

Figure 46. Microgrid load profile (Butt 2011). ${ }^{142}$

\subsubsection{Step 3: Classify DER (Butt 2011) -see Figure 47}

"Distributed generation and energy storage:

- Coverage required for microgrid footprint;

- Existing standby generation characteristics;

- Proposed/funded generation projects;

- Fuel inventory and duration requirements;

- Dispatchability to intermittency ratio; and

- Distributed storage considerations - location, technology, capacity, and duration" (Butt 2011).

\footnotetext{
${ }^{142}$ Reused by permission of NREL.
} 


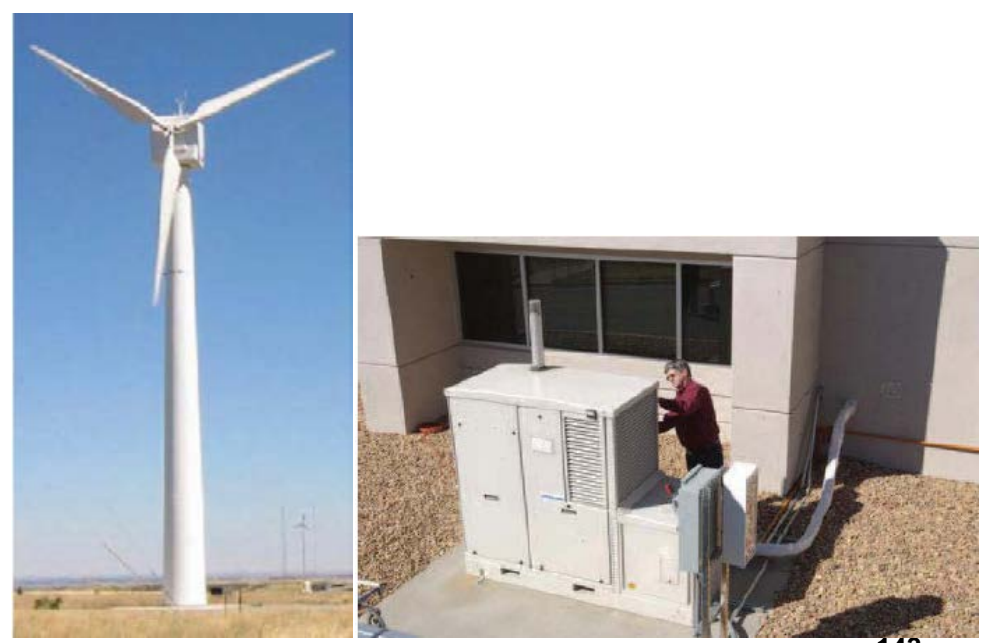

Figure 47. Wind turbine and fuel cell (Butt 2011). ${ }^{143}$

\subsubsection{Step 4: Evaluate generation/load match (Butt 2011)}

- "Can existing/projected generation capacity meet the proposed peak load and daily operating requirements?

- Can dispatchable resources handle transient disturbances on the system while maintaining satisfactory voltage and frequency?

- Can existing dispatchable generation compensate for the variability of renewable resources?" (Butt 2011)

\subsubsection{Step 5: Develop control strategy (Butt 2011)}

- "Appropriate control strategies for self regulation - load and generation dispatch;

- Include both grid-connected and islanded condition;

- Control of utility interface (i.e., static transfer switch) to handle seamless separation and reconnection to utility power;

- Utility requirements (monitoring and/or control of interface);

- Local /central control schemes to monitor and control distributed sources and loads;

- Interface with building energy management system(s);

- Integration with existing legacy communication systems/software;

- Address security concerns - cyber and physical; and

- Integration with existing protection schemes" (Butt 2011).

\footnotetext{
${ }^{143}$ Reused by permission of NREL.
} 
10.2.2.6 Step 6: Determine equipment requirements (Butt 2011)

"Engineering analysis includes modeling and simulation of the microgrid under various scenarios to establish necessary control parameters, devices, infrastructure modifications, and additional generation and storage necessary for microgrid implementation.

Assist with RFP development that specifies site microgrid requirements" (Butt 2011). 


\section{Existing Regional VG Planning Practices in the Industry ${ }^{144}$}

The following sections outline similar guidelines as adopted by different regulations organizations. These guidelines are relevant to planning, operation and control, and/or market operation.

\subsection{U.S. Planning Guidelines}

\subsubsection{California ISO Interconnection Standards Review Initiative}

"California independent system operator (CAISO) has commenced this initiative to promote the continued reliability of the ISO-controlled grid by refining a limited number of interconnection standards for new large generating facilities, i.e., those facilities that area equal to or greater than $20 \mathrm{MW}$. The ISO has over $21,000 \mathrm{MW}$ of variable generation capacity in the 'serial group' and 'transition cluster' portions of its interconnection queue"145 (CAISO 2010).

\subsubsection{Power factor requirements for asynchronous VER plants}

"The ISO proposes to require asynchronous VERs to have the capability of providing reactive power output within 0.95 lag to 0.95 lead as measured at the Point of Interconnection (POI). This requirement ensures that the VER provides sufficient reactive power to maintain a specified voltage schedule in accordance with the ISO Tariff.

"This 0.95 lag to 0.95 lead design recommendation is consistent with the capabilities already imposed on wind facilities under Appendix $\mathrm{H}$ of the LGIA pursuant to FERC Order No. 661-A. However, the ISO proposes the following key revisions related to this recommendation:

(a) The power factor requirements (0.95 lag to 0.95 lead) shall apply to all asynchronous VERS.

(b) No reactive support will be required if the VER is exporting less than 20 percent of the maximum rated power to the POI.

(c) If the VER is exporting 20 percent or more of the maximum rated power, it should be capable of providing reactive support. The maximum amount of reactive support will be determined by the amount of power exported to the POI. For example, a VER is exporting $10 \mathrm{MW}$ to the POI. The VER should be capable of injecting or absorbing up to 3.3 Mvar at the POI.

(d) The requirement will apply without the need to perform an interconnection study

"The ISO believes that extending the existing Order No. 661-A beyond wind facilities to solar photovoltaic generators, or other asynchronous technologies, is appropriate given the similarity in power converter systems used by such technologies. Asynchronous VERs, including wind, utilize inverter-based technology to deliver power to the ISO grid in a synchronous manner. Also, both wind and solar photovoltaic facilities consist of multiple generating units. The extension of Order No. 661-A from wind to all VERs is a logical progression.

\footnotetext{
${ }^{144}$ Contributed by Yuri Makarov, PNNL.

145 The serial group has over 12,000 MW of renewable resource capacity, while the transition cluster has over 9,000 MW of renewable resource capacity.
} 
"This requirement will not apply to any asynchronous generating facility that has an executed LGIA or has been tendered an LGIA as of the date this proposal is approved by the ISO Board of Governors. In addition, the requirement will not extend to any wind facility in the 'serial group,' without an LGIA or tendered LGIA, to the extent it was not required by a completed system impact study to provide 0.95 lead/lag power factor. Further, to the extent an asynchronous generating facility has executed an LGIA that provides for the existing 0.90 lag and 0.95 lead requirement that is applicable to synchronous machines, the interconnection customer may inform the ISO that it elects to comply with the revised requirement.

"The reactive power sources needed to comply with this design requirement can be provided by:

1. The inverters associated with the asynchronous generation;

2. Switched or fixed capacitors;

3. Static devices (such as a STATCOM); or

4. A combination of these sources.

Capacitors have been used extensively for many years to provide reactive power support to the power system. Capacitors are readily available and the application to the power grid is well understood by power system engineers. As such, applying the requirement is not expected to result in material engineering changes by the developer, delays in permitting, or the need to delay or redo any existing ISO interconnection study. Nor does the ISO believe that the presence of pre-existing power purchase agreement justifies an exemption. Notwithstanding the outcome of Nevada Power Company, FERC Docket No. ER10-508-000 (February 10, 2010), solar facilities that are required under the ISO Tariff to meet the 'existing power factor requirement and wind facilities without a completed system impact study,' or a Phase II cluster interconnection study remained potentially subject to a power factor requirement under Order No. 661-A. It follows that for projects in the foregoing situations, the power purchase agreement should have taken these potential costs into consideration.

"The ISO is aware that Order 661-A and Nevada Power Company place the burden on the transmission operator to establish the need for a power factor requirement. The ISO's 2007 Integration of Renewable Resources study, based on transient and post-transient analyses, concluded that "all new wind generation units must have the capability to meet the WECC requirements of \pm 0.95 power factor. This reactive capability is essential for adequate voltage control." This conclusion as well as other subsequent analyses forms the basis of the ISO's recommendation to make the power factor requirement mandatory" (CAISO 2010b).

\subsubsection{Voltage regulation requirements}

"Article 9.6.2 of LGIA establishes the requirement for an Interconnection Customer to maintain Voltage Schedules. This is applicable to "all" generators, conventional and VERs. In order to reliably operate the transmission system within an acceptable voltage range at the POI, the ISO proposes that all new generators connecting to the ISO grid adhere to these requirements. The requirements below outline some specifics related to designing the voltage regulation mechanism:

- Install an Automatic Voltage Control system so that the generating facility can help regulate the transmission voltage at the $\mathrm{POI}$ both under steady state and disturbance conditions, as per the voltage schedule provided. 
- All reactive power devices used to vary the generating facility's reactive power output should be under the control of the Automatic Voltage Control system.

- Coordinate with the Participating Transmission Owner (PTO) and the ISO for voltage schedule requirements at the POI.

- The Automatic Voltage Control system will normally be required to regulate the voltage at the POI. However, in some circumstances, it may be more efficient or necessary, as determined by the CAISO in coordination with the PTO, to regulate the voltage at a point on the generator's side of the POI. Note: regulating voltage to a point other than the POI will not change the power factor requirements, i.e., the VER is still required to have sufficient reactive capability to maintain a $0.95 \mathrm{lag} /$ lead power factor at the POI.

- $\quad$ The VER will not be required to incrementally produce or absorb Vars (reactive power) if such operation requires the VER to operate in a voltage range outside of the continuous operating limits (generally accepted to be 0.9 PU $<=\mathrm{V}<=1.10 \mathrm{PU}$ ) at the generator terminals. The plant shall be designed so that the VER can provide reactive support when the voltage at the POI is 0.95 PU $<=V<=1.05 \mathrm{PU}$. Note: PU is defined as Per Unit voltage.

Each generation facility should design its systems so that it can be operated in either a voltage control mode or a power factor control mode. Based on the voltage schedule or power factor set point provided, the generation facility should be able to produce / absorb VARs in such a fashion as to meet the desired voltage / power factor setting at the POI. The normal mode of operation for generators is voltage control mode" (CAISO 2010).

\subsubsection{Ride-through capability requirements}

"ISO recommends designing all new generation facilities so that it can comply with the substantive voltage ride-through criteria as specified in draft 2 of NERC Standard PRC-024-1" (CAISO 2010).

\subsubsection{Frequency ride-through capability}

"The ISO recommends new projects to be designed to be compliant with meeting WECC offnormal frequency (ONF) plan requirements, rather than requirements in NERC PRC-024-1... WECC is aware of this potential conflict, and intends to seek a regional variance from NERC PRC-024-1 standard for frequency ride-through requirements" (CAISO 2010).

\subsubsection{Generator power management requirements}

"The ISO tariff requires any market participant, including a participating generator, to comply fully and promptly with ISO dispatching instruction or operating orders. These requirements exist to ensure that the ISO has sufficient control over equipment interconnected to its transmission system to maintain reliability under normal and emergency operating conditions. Innovation in pitch control of wind turbines, as well as the advanced power control systems of many original equipment manufacturers (OEMs), now permits VG to control power output and contribute to system reliability when necessary. The ISO recognizes, however, that actively controlling VG output involves the spilling of wind or sun resource and therefore must be utilized in a very judicious manner consistent with market efficiency and environmental objectives. This 
initiative is limited to providing basic capability requirements for power management that promote grid reliability and do not foreclose future market design options.

"All are required to provide the following power management features:

- Active power management;

- Ramp rate limits and control; and

- Over-frequency response" (CAISO 2010b).

Additional information on generator power management requirements is provided in Appendix 27.

\subsubsection{Bonneville Power Administration (BPA) ${ }^{146}$}

"BPA's balancing responsibilities have become more challenging in light of the rapidly growing number of wind turbines that have been installed in the Pacific Northwest during the past decade. Installed wind capacity in the Pacific Northwest has grown from nearly no wind power in 2000 , to nearly $6,000 \mathrm{MW}$ in 2010 (IEA Wind 2011). As of 2010, BPA had approximately 2,800 MW of wind facilities on its system (BPA 2010c). BPA has offered about 9,300 MW of transmission to existing or planned wind projects (IEA Wind 2011), and BPA forecasts that by 2020, the Northwest will have over 14,000 MW of wind power (IEA Wind 2011).

"At the same time the installed wind power capacity on BPA's system is growing, the capacity factor of the wind projects on BPA's system is declining from an average of 33.1 percent in 2008 to 27.1 percent in 2010. This is not surprising since the best wind project sites, where wind is most constant, were presumably developed first.

BPA's peak load is roughly 10,500 MW. Measured by the percentage of installed wind capacity on various BA systems, BPA's wind penetration by 2010 was roughly 31 percent of its peak loads (as compared to 9 percent for Electric Reliability Council of Texas (ERCOT) and 6 percent for the California ISO (CAISO) (BPA 2009).

Recognizing that a great deal of wind power was under development in the Pacific Northwest, the Northwest Power Planning and Conservation Council (the Council) released its Fifth Power Plan in 2005, which called for up to $6,000 \mathrm{MW}$ of regional wind development over the next 20 years. In response, BPA and the Council co-sponsored the Northwest Wind Integration Action Plan (Action Plan). ${ }^{147}$ This collaborative effort brought together stakeholders to determine the technical feasibility of integrating $6,000 \mathrm{MW}$ of wind energy into the Northwest grid. The Action Plan also identified what it would take, in infrastructure investment, operational changes, costrecovery, and technology innovation to integrate the Northwest's rapidly growing wind resources in a reliable and cost-effective fashion (BPA 2010a).

The Action Plan also identified the importance of mechanisms to recover the costs of wind integration as a threshold issue. In 2007, BPA proposed a new rate to recover the costs of carrying capacity reserves to balance the within-hour variability of wind. BPA has historically relied on the Federal Columbia River Power System (FCRPS) to provide the capacity needed to

\footnotetext{
${ }^{146}$ Contributed by Jian Ma, PNNL.

147 The Northwest Wind Integrstion Action Plan, March 2007. Available http://www.nwcouncil.org/energy/wind/library/2007-1.pdf Accessed 12/6/2012.
} 
balance variation in wind generation within the hour. BPA generally refers to the capacity reserve needed to account for within-hour variability as "balancing reserve capacity." The largescale export of wind generation out of the BPA BA has placed enormous pressure on the balancing capability of the FCRPS. That's because BPA must stand ready to provide balancing reserve capacity and energy for large quantities of wind exported to meet demand in other BAs.

When the large, geographically concentrated wind fleet in BPA's BA experiences a significant ramp event (up or down), BPA sees substantial variations between the wind hourly schedules and the actual performance of the generators, which contributes substantially to the need for balancing reserve capacity. This balancing reserve capacity includes three components:

1. Regulation reserve (moment to moment balancing);

2. Following reserve (moment to 10-minute variation balancing), and

3. Imbalance reserve (balancing the difference between schedule and actual for the hour).

In 2009, BPA needed $203 \mathrm{MW}$ of reserve generation to integrate wind resources (BPA 2008a).

BPA's resulting wind integration - within-hour balancing service rate was designed to recover the costs BPA incurs as a result of reserves required to address within-hour variability of wind generation. Traditionally, within-hour variability has been the result of changes in loads (i.e., demand), and BPA has accordingly allocated the costs of its following reserves (moment to 10minute variation balancing) to the rate charged to customer-owned utilities. Because the majority of wind generated in the Northwest is exported to consumers outside of BPA's BA area, BPA declined to allocate its wind integration charge to rates. Instead, the wind integrationwithin-hour balancing service rate was designed as a per-kW charge on wind generators based on the amount of its installed capacity.

\subsubsection{Midwest ISO Multi-Value-Based Planning Process}

Midwest ISO (MISO) has transformed its transmission expansion planning model. The previous reliability-based model had the following main characteristics:

- Focused primarily on grid reliability;

- Typically considered a short-time horizon; and

- Sought to minimize transmission build (Bear 2011).

\subsubsection{Value based planning methodology and multi-value projects}

"Now the Midwest ISO follows a value-based planning methodology. This methodology indicates a transition from reliability-based planning model to the multi-value-based model.

Multi-value projects (MVP) create regional network that, when combined with the existing system, provides value in excess of its costs under a variety of future policy and economic conditions (Midwest ISO 2011). The new multi-value-based model:

- Focuses on value while maintaining reliability;

- Reflects appropriate project time scales;

- Seeks to identify transmission infrastructure that maximizes value, and

- Identifies the comprehensive value of projects (Bear 2011). 
"Multi-value projects must meet one of the three, tariff-defined criteria:

- Criterion 1: A MVP must be developed through the transmission expansion planning process to enable the transmission system to deliver energy reliably and economically in support of documented energy policy mandates or laws enacted or adopted through state or federal legislation or regulatory requirement. These laws must directly or indirectly govern the minimum or maximum amount of energy that can be generated. The MVP must be shown to enable the transmission system to deliver such energy in a manner that is more reliable and/or more economic than it otherwise would be without the transmission upgrade.

- Criterion 2: A MVP must provide multiple types of economic value across multiple pricing zones with a total MVP benefit to cost ratio of 1.0 or higher. The reduction of production costs and the associated reduction of LMPs from a transmission congestion relief project are not additive, and are considered a single type of economic value.

- Criterion 3: A MVP must address at least one transmission issue associated with a projected violation of a NERC or regional standard and at least one, economic-based transmission issue that provides economic value across multiple pricing zones. The project must generate total, financially quantifiable benefits, including quantifiable reliability benefits, in excess of the total project" (Midwest ISO 2011).

"The MISO tariff also identifies specific types of economic value, which MVP may provide, including:

- Production cost savings, where production costs include generator startup, hourly generator no-load, generator energy, and generator operating reserve costs. Production cost savings can be realized through reductions in both transmission congestion and transmission energy losses. Production cost savings also can be realized through reductions in operating reserve requirements within reserve zones and, in some cases, reductions in overall operating reserve requirements for the transmission provider.

- Capacity losses savings, where capacity losses represent the amount of capacity required to serve transmission losses during the system peak hour, including associated planning reserve.

- Capacity savings due to reductions in the overall planning reserve margins, resulting from transmission expansion.

- Long-term cost savings realized by transmission customers, by accelerating a long-term project start date in lieu of implementing a short-term project in the interim, and/or longterm cost savings realized by transmission customers by deferring or eliminating the need to perform one or more projects in the future.

- Any other financially quantifiable benefit to transmission customers, resulting from an enhancement to the transmission system and related to the provisions of transmission service" (Midwest ISO 2011).

Table 13 contains the scope of the candidate MVP portfolio analysis (Midwest ISO 2011). 
Table 13. The scope of the MVP portfolio analysis (Midwest ISO 2011). ${ }^{148}$

\begin{tabular}{|c|c|}
\hline Analysis Type & Analysis Output \\
\hline Steady state & List of thermal overloads mitigated by the proposed MVP portfolio transmission projects \\
\hline Alternatives & $\begin{array}{l}\text { Relative value of the candidate MVP projects against a stakeholder or MISO identified altemative } \\
\text { Can include steady state and production cost analyses }\end{array}$ \\
\hline $\begin{array}{l}\text { Underbuild } \\
\text { requirements }\end{array}$ & $\begin{array}{l}\text { Document any incremental transmission required to mitigate constraints created by the addition of } \\
\text { the proposed MVP portfolio }\end{array}$ \\
\hline Short circuit & Document any incremental upgrades required to mitigate any short circuit / breaker duty violations \\
\hline Stability & $\begin{array}{l}\text { List of violations mitigated by the proposed MVP portfolio transmission projects } \\
\text { Includes both transient and voltage stability analysis }\end{array}$ \\
\hline Generation enabled & Document wind curtailed, and additional wind that is enabled by the proposed MVP portfolio \\
\hline Production cost & Adjusted Production Cost (APC) benefits of the entire proposed MVP portfolio \\
\hline Robustness testing & Quantification of portfolio benefits under various policy futures or transmission conditions \\
\hline $\begin{array}{l}\text { Operating reserves } \\
\text { Impact }\end{array}$ & $\begin{array}{l}\text { Impact of the proposed MVP portfolio on existing operating reserve zones and quantification of } \\
\text { this benefit }\end{array}$ \\
\hline $\begin{array}{l}\text { Planning Reserve } \\
\text { Margin (PRM) benefits }\end{array}$ & $\begin{array}{l}\text { Capacity savings due to reductions in the system wide Planning Reserve Margin caused by the } \\
\text { addition of the proposed MVP portfolio to the transmission system }\end{array}$ \\
\hline $\begin{array}{l}\text { Transmission loss } \\
\text { reductions }\end{array}$ & $\begin{array}{l}\text { Capacity losses savings, where capacity losses represent the amount of capacity required to } \\
\text { serve transmission losses during the system peak hour }\end{array}$ \\
\hline $\begin{array}{l}\text { Wind generation } \\
\text { capital investment }\end{array}$ & $\begin{array}{l}\text { Quantification of the incremental wind generator capital cost savings enabled by the wind siting } \\
\text { methodology supported by the proposed MVP portfolio }\end{array}$ \\
\hline $\begin{array}{l}\text { Transmission capital } \\
\text { investment }\end{array}$ & $\begin{array}{l}\text { Document the future baseline transmission investment that may be avoided due to the installation } \\
\text { of the proposed MVP portfolio }\end{array}$ \\
\hline
\end{tabular}

\subsubsection{MVP portfolio reliability and public policy benefits}

\section{Steady-state analyses}

"A series of steady-state analyses are conducted to determine the transmission line overloads and system voltage constraints mitigated by the proposed MVP portfolio.

- This analysis also ensures that the system reliability is maintained with the inclusion of the MVP portfolio into the transmission system.

- Any new violations with the MVP portfolio are identified, and transmission upgrades are included in the MVP portfolio to resolve these issues.

- $\quad$ The proposed MVP portfolio maintains system reliability by resolving:

o Thermal overloads for approximately 6,400 system conditions; and

o Voltage violations for approximately 300 system conditions" (Midwest ISO 2011).

\section{Transient stability}

"Additional analyses are performed to analyze the benefits of the MVP portfolio on transient stability" (Midwest ISO 2011). "The proposed MVP portfolio mitigates 31 fault conditions that could cause system instability" (Midwest ISO 2011).

\section{Voltage stability}

\footnotetext{
${ }^{148}$ Reused by permission of MISO.
} 
"Voltage stability analyses were performed to voltage collapse conditions under high-energy transfer conditions from major generation resources to major load sinks.

- Such transfers may occur during critical dispatch scenarios, such as when local area generation near large load centers are offline, and remote generation resources are supplying energy to the load centers.

- Additional transfer capacity will increase system reliability and robustness, allowing additional energy sources to be dispatched to serve load centers as needed.

- The proposed MVP portfolio causes an increase in transfer capability from wind rich regions by 5,341 MW" (Midwest ISO 2011).

\section{Renewable energy}

"The proposed MVP portfolio reliably enables the delivery of energy in support of policy mandates:

- Through resolving reliability constraints that would otherwise result in the curtailment of wind generation, the proposed MVP portfolio will reliably enable the states in the MISO footprint to meet their renewable energy mandates.

- The proposed MVP portfolio enables the delivery of 41 million MWh of renewable energy annually.

- This energy will support the renewable energy mandates of the MISO states through at least 2026" (Midwest ISO 2011).

\subsubsection{Economic variables and assumptions}

\section{Future policy scenarios}

"Scenario-based planning is also a key element, allowing identification of transmission that is needed to meet a variety of potential generation outcomes. This identifies the "no regrets" transmission that can be built while the energy policy is still evolving" (Bear 2011).

"To ensure that the proposed MVP portfolio delivers benefits under a variety of future assumptions, several different future scenarios were analyzed:

- Business as usual with continued low demand and energy growth assumes that current energy policies will be continued, with continuing, recession-level low demand and energy growth projections.

- Business as usual with historic demand and energy growth assumes that current energy policies will be continued, with demand and energy returning to pre-recession growth rates.

- Carbon constrained assumes that current energy policies will be continued, with the addition of a carbon cap modeled on the Waxman-Markey bill. 
- Combined energy policy assumes a myriad of energy policies are enacted, including a 20 percent federal renewables portfolio standard (RPS), a carbon cap modeled on the Waxman-Markey bill, the implementation of a smart grid, and the widespread adoption of electric vehicles.

The majority of the analysis focused on the 'Business as usual' scenarios, although sensitivities were completed to determine the benefits of the proposed MVP portfolio under scenarios with new energy policies" (Midwest ISO 2011).

\section{Discount rate and timeframe}

"Time horizon is 20 to 40 years from portfolio in-service date. Overhead transmission lines are projected to have a total lifespan of 70 to 80 years. The tariff typically refers to a 20 -year time horizon for Benefit/Cost (B/C) calculations. A 40-year time span allows additional benefits to be captured for each transmission facility, without requiring extremely long range projections. It also matches the assumed book life of the facilities. Discount rate is assumed to be between 3.00 and 8.2 percent. The social discount rate of 3.00 percent is roughly equal to the value a typical rate payer would be able to receive on their risk-adjusted investment. The weighted average cost of capital (WACC) of 8.2 percent was calculated by a gross transmission plantweighted transmission owner cost of capital. This reflects the minimum return the transmission owners must receive to satisfy their creditors, owners, and other providers of capital. These variables were adjusted in the base business case calculations" (Midwest ISO 2011).

\section{Additional business case variables}

Wind turbine capital cost: $\$ 2.0$ to $\$ 2.9$ million/MW.

- $\quad$ Based upon the U.S. Energy Information Administration's capital cost estimates for onshore wind (updated November 2010).

- This variable is adjusted in the base business case analysis.

Operating reserve optimization benefit: $\$ 5$ to $\$ 7 / \mathrm{MWh}$.

- Based upon changes in day-ahead market clearing prices for June 1, 2010 through May 31, 2011.

- This variable is adjusted in the base business case analysis.

Natural gas prices:

- Business as usual scenarios: $\$ 5$ to $\$ 8 / M M B t u$.

- Carbon and combined policy scenarios: $\$ 8$ to $\$ 10 / M M B t u$.

- A natural gas price of $\$ 5$ was used for the base business case analysis. Higher natural gas prices were used as sensitivities" (Midwest ISO 2011).

European planning guidelines are provided in Appendix 28. 


\section{Bibliography}

ABB. 2012. "HVDC and HVDC Light." Montreal, Canada. Available http://www.abb.com/hvdc. Accessed November 26, 2012.

ABB. 2003. "Blackout and Electricity Stability." ABB, Montreal, Canada. Available http://www.google.com/url?sa=t\&rct=j\&q=\&esrc=s\&frm=1\&source=web\&cd=1\&cad=rja\&ved=0C DAQFjAA\&url=http\%3A\%2F\%2Fwww02.abb.com\%2Fdb\%2Fdb0003\%2Fdb002698.nsf\%2Fca7 e93ab03030d22c12571380039e8fc\%2F1c749593e024afc8c12571da0032d456\%2F\%24FILE\% 2FFacts\%2Bwhite\%2Bpaper\%2BFinal.doc\&ei=rJedUKvtB-

SKjAKD0oCYCw\&usg=AFQjCNHe5dAcHi9MZypRiLt0xiaAUJhLdA\&sig2=i3QHHK19720zLMief a wRQ. Accessed November 28, 2012.

Abdelaziz, A.R. 1999. "A Fuzzy-Based Power System Reliability Evaluation." Electric Power Systems Research, vol. 50, pp. 1-5.

Abraham, K.M., and Z. Jiang. 1996. "A Polymer Electrolyte Based Rechargeable Lithium/Oxygen Battery." J. Electrochem. Soc., vol. 143, no. 1, pp. 1-5.

Abreu, L.V.L., and M. Shahidehpour. 2006. "Wind Energy and Power System Inertia." IEEE Power Engineering Society General Meeting, Montreal, Quebec, 2006. Institute of Electrical and Electronics Engineers, Piscataway, NJ.

Adamczyk, A., R. Teodorescu, R. N. Mukerjee, and P. Rodriguez. 2010. "Overview of FACTS Devices for Wind Power Plants Directly Connected to the Transmission Network." 2010 IEEE International Symposium on Industrial Electronics (ISIE), pp. 3742-3748, July 4-7, 2010. Institute of Electrical and Electronics Engineers, Piscataway, NJ.

Adler, T., F. McLarnon et al., undated. "Low Zinc Solubility Electrolytes for Use in Zinc/Nickel Oxide Cells." J. Electrochem. Soc., vol. 140, no. 2, pp. 289-294.

Agreira, C.I.F., S. M. F. de Jesus, S. L. de Figueiredo, C. M. Ferreira, J. A. D. Pinto, and F. P. M. Barbosa. 2006. "Probabilistic Steady-State Security Assessment of an Electric Power System Using a Monte Carlo Approach." In Proceedings of the 41st International Universities Power Engineering Conference, pp. 408-411, 2006. Institute of Electrical and Electronics Engineers, Piscataway, NJ.

Ajjarapu, V. and B. Lee. 1998. "Bibliography on Voltage Stability." IEEE Transactions on Power Systems, vol. 13, pp. 115-125, February 1998.

Alberta Energy. 2009. "Assessment and Analysis of the State-Of-the-Art Electric Transmission Systems with Specific Focus on High-Voltage Direct Current (HVDC), Underground or Other New or Developing Technologies." Alberta Energy, Edmonton, Alberta, Canada. December 23, 2009. Available http://www.energy.alberta.ca/Electricity/pdfs/TransmissionSystemsStudy.pdf.

Alberto, L.D.C., F.H.J.R. Silva, and N.G. Bretas. 2001. "Direct Methods for Transient Stability Analysis in Power Systems: State of Art and Future Perspectives." In Proceedings of IEEE Porto Power Tech Proceedings, vol. 2, September 10-13, 2001. Institute of Electrical and Electronics Engineers, Piscataway, NJ. 
Aldea, C., and S. C. Savulescu. 2004. "Evaluation of the Stability Reserve of Transelectrica's Transmission System by Using Quickstab Professional." In Proceedings of National Conference of Energy CNE, Neptun, Romania, June 2004, pp. 1-6.

Allan, R.N., R. Billinton, A. M. Briepohl, and C. H. Grigg. 1994. "Bibliography on the Application of Probability Methods in Power System Reliability Evaluation, 1987-1991." IEEE Transactions on Power Systems, vol. 9, pp. 41-49, 1994.

Allan, R.N., R. Billinton, A. M. Breipohl, and C. Griss. 1999. "Bibliography on the Application of Probability Methods in Power System Reliability Evaluation, 1992-1996." IEEE Transactions on Power Systems, vol. 14, pp. 51-57, 1999.

Allan, R.N., R. Billinton, and S. H. Lee. 1984. "Bibliography on the Application of Probability Methods in Power System Reliability Evaluation, 1977-1982." IEEE Transactions on Power Apparatus and Systems, vol. PAS-103, pp. 275-282, 1984.

Allan, R.N., R. Billinton, S. M. Shahidehpour, and C. Singh. 1988. "Bibliography on the Application of Probability Methods in Power System Reliability Evaluation, 1982-1987." IEEE Transactions on Power Systems, vol. 3, pp. 1555-1564, 1988.

Allan, R.N., A. M. L. Da Silva, and R. C. Burchett. 1981. "Evaluation Methods and Accuracy in Probabilistic Load Flow Solutions." IEEE Transactions Power App. Syst., vol. PAS-100, pp. 2539-2546, May.

Alstom Grid. 2010. Multi-terminal HVDC System for Large Offshore Wind Park Grid Integration, Alstom Grid, Summer 2010, pp. 24-26. Available http://www.alstom.com/Global/Grid/Resources/Documents/Smart\%20Grid/Think\%207\%20\%2009\%20chapter\%202\%20\%20Art\%201\%20Multi\%20terminal\%20HVDC\%20system\%20.pdf.

Anaya-Lara, O., F.M. Hughes, N. Jenkins, et al. 2006. "Influence of Wind Farms on Power System Dynamic and Transient Stability." Wind Engineering, vol. 30, no. 2, pp. 107-27.

Anderson, P.M., and B.K. LeReverend. 1996. "Industry Experience with Special Protection Schemes." IEEE Transactions on Power Systems, vol. 11, no. 3, August.

Angeles-Camacho, C., O.L. Tortelli, E. Acha, and C.R. Fuerte-Esquivel. 2003. "Inclusion of a High Voltage DC Voltage Source Converter Model in a Newton-Raphson Power Flow Algorithm." IEE Proceedings of Generation, Transmission, and Distribution, vol. 150, no. 6, pp. 691-696.

Ankan, K. Deb. 2000. Powerline Ampacity System: Theory, Modeling, and Applications, CRC Press, Boca Raton, FL.

Arges, C.G., V. Ramani, and P.N. Pintauro. 2010. "Anion Exchange Membrane Fuel Cells." The Electrochemical Society Interface, pp. 31-35.

Armand, M., and J.M. Tarascon. 2008. "Building Better Batteries." Nature Materials, vol. 451, pp. 652-657. 
Arrillaga, J., Y.H. Liu, and N.R. Watson. 2007. Flexible Power Transmission. The HVDC Options, J. Wiley and Sons Ltd, Hoboken, NJ. .

Asare, P.O., A.W. Galli, J.S. Lee, C.A. Lozano, and E.O'Neil-Carillo. 1995. Real-Time Pricing of Electric Power.ECE Technical Report, Paper 120, Purdue University, West Lafayette, NJ. Available http://docs.lib.purdue.edu/cgi/viewcontent.cgi?article=1121\&context=ecetr. Accessed November 28, 2012.

Asmine, M., J. Brochu, J. Fortmann, R. Gagnon, Y. Kazachkov, C. E. Langlois, C. Larose, E. Muljadi, J. MacDowell, P. Pourbeik, S. A. Seman, and K. Wiens. 2011. "Model Validation for Wind Turbine Generator Models." IEEE Transactions on Power Systems, pp. 1769-1782, August.

Asplund, G. 2007. "Ultra-High Voltage Transmission." ABB Review February 2007, pp. 22-27. Available http://www05.abb.com/global/scot/scot271.nsf/veritydisplay/9e16e26d65ab7339c12572fe004de b21/\$file/22-27\%202m733 eng72dpi.pdf. Accessed November 20, 2012.

Asplund, G. 2000. "Application of HVDC Light to Power System Enhancement." In Proceedings of IEEE PES Winter Meeting 2000, Singapore, January 23-27, 2000. Institute of Electrical and Electronics Engineers, Piscataway, NJ.

Asplund, G., and A. Williamson. 2007. "A Novel Approach to Providing on Route Power Supplies to Rural and Urban Communities in Close Proximity to the Extra High-Voltage DC Transmission Line." IEEE PES Power Africa 2007 Conference and Exposition, South Africa, July 2007. Institute of Electrical and Electronics Engineers, Piscataway, NJ.

Athay, T., R. Podmore, and S. Virmani. 1979. "A Practical Method for the Direct Analysis of Transient Stability." IEEE Transactions on Power Apparatus and Systems, vol. PAS-98, no. 2, pp. 573-584, March.

Atlantic Wind Connection. 2012. Atlantic Wind Connection website. Atlantic Grid Development LLC, Chevy Chase, MD. Available http://atlanticwindconnection.com/awc-intro/. Accessed November 15, 2012.

Austria, R.R., X. Y. Chao, N. P. Reppen, and D. E. Welsh. 1995. "Integrated Approach to Transfer Limit Calculations." Proceedings of IEEE Computer Applications in Power, January 1995, pp. 48-52. Institute of Electrical and Electronics Engineers, Piscataway, NJ.

Aven, T. 2008. Risk Analysis Assessing Uncertainties beyond Expected Values and Probabilities: John Wiley \& Sons Ltd., Hoboken, NJ, 2008.

Axion Power International Inc. 2011. "Axion Power's PowerCube Battery Energy Storage System Integrated into PJM Utility Grid." November. Available http://www.eetimes.com/electronics-products/electronic-product-releases/smart-energydesign/4231963/Axion-Power-s-\%20PowerCube-Battery-Energy-Storage-System-IntegratedInto-PJM-Utility-Grid. Accessed November 12, 2012.

Bagnall, T., C. Ritter, B. Ronner, P. Maibach, N. Butcher, and T. Thurnherr. 2009. "PCS6000 STATCOM Ancillary Functions: Wind Park Resonance Damping." EWEC, Maeseille. 
Bahrman, M.P., J. G. Johansson, and B. A. Nilsson. 2003. "Voltage Source Converter Transmission Technologies: The Right Fit for the Application." IEEE Power Engineering Society General Meeting, 13-17, July 2003. Institute of Electrical and Electronics Engineers, Piscataway, NJ. Available http://ieeexplore.ieee.org/stamp/stamp.jsp?tp=\&arnumber=1267441\&isnumber=28353. Accessed November 15, 2012.

Balducci, A. et al. "Cycling Stability of a Hybrid-Activated Carbon//poly(3-methylthiophene) Supercapacitor with N-butyl-N-methylpyrrolidinium Bis(trifluoromethanesulfonyl)imide Ionic Liquids as electrolyte." Electrochim Acta 50 (2005) 2233-2237.

Baran, M.E., S. Teleke, L. Anderson, S. Bhattacharya, A. Huang, and S. Atcitty. 2008. "STATCOM with Energy Storage for Smoothing Intermittent Wind Farm Power." IEEE/PES, Institute of Electrical and Electronics Engineers, Piscataway, NJ.

Bauer, P. 1968. "Batteries for Space Power Systems." ed. NASA, Washington, D.C. Vol. NASA SP-172.

BC Hydro. 2006. Voltage Stability Operating Limits and Procedures for Using the RT-VSA and VSA Applications, System Operating Order 7T - 50, Real Time Operations, British Columbia Transmission Corporation, Vancouver, BC, Canada. May 2, 2006. Available http://www.bctc.com/NR/rdonlyres/BD53F0FD-A80A-4D0A-B4B9-11D13EFB9566/0/7T50.pdf.

BCP Switzerland. undated. NEPLAN Electricity - Voltage Stability. Available http://www.neplan.ch/html/e/pdf e/neplan t03 voltagestability engl.pdf. Accessed November $27,2012$.

Beacon Power, "Frequency Regulation and the Smart Energy Matrix." Beacon Power, Tyngsboro, MA. Available http://www.beaconpower.com/products/flywheel-energy-storage.asp.

Bear, J. 2011. "Renewable Integration: The Story So Far and What Lies Ahead." MISO Presentation, Utility Scale Flexible Power Summit, Denver, CO, September 27, 2011. Midwest ISO, Carmel, IN. Available http://www.greenpowerconferences.com/EF/Events/RE1109US/docs/1/John Bear.pdf. Accessed November 5, 2012.

Bebic, J. 2008. Power System Planning: Emerging Practices Suitable for Evaluating the Impact of High-Penetration Photovoltaics, NREL, Golden, CO. February. Available http://www1.eere.energy.gov/solar/pdfs/42297.pdf. Accessed November 27, 2012.

Bebic, J., R. Walling, K. O'Brien, and B. Kroposki. 2009. "The Sun Also Rises." IEEE Power and Energy Magazine, vol. 7, no. 3, May 2, 2009, pp. 45-54.

Begovic, M.M. and A.G. Phadke. 1992. "Control of Voltage Stability Using Sensitivity Analysis." IEEE Trans. on Power Syst., vol. 7, no. 1, February 1992, pp. 114-123.

Behr, P. 2011. "Germany Sees Thousands of Miles of New Power Lines on Its Road to Renewable Energy." New York Times, August 1, 2011. Available http://www.nytimes.com/cwire/2011/08/01/01climatewire-germany-sees-thousands-of-miles-ofnew-power-43095.html?pagewanted=all. Accessed November 20, 2012. 
Belanger, D., T. Brousse, and J.W. Long. 2008. "Manganese Oxides: Battery Materials Make the Leap to Electrochemical Capacitors." The Electrochemical Society Interface, Spring: pp. 4952.

Bents, D.J. et. al. 2005. Hydrogen-Oxygen PEM Regenerative Fuel Cell Energy Storage System. NASA, Washington, D.C.

Bessette, N. 2011. "Development of a Low-Cost 3-10 kW Tubular SOFC Power System." DOE Hydrogen and Fuel Cells Program Annual Progress Report, DOE, Washington, D.C. Editor. Acumentrics Corporation. Westwood, MA. pp. 890-893.

Bettiol, A.L., Y. Zhang, L. Wehenkel, and M. Pavella, 1997. "Transient Stability Investigations on a Brazilian Network by SIME." In Proceedings of Fourth International Conference on Advances in Power System Control, Operation and Management APSCOM-97, paper 450, vol. 1, pp. L1L6, November 11-14, 1997, Institute of Electrical and Electronics Engineers, Piscataway, NJ.

Bevrani, H., and Takashi Hiyama. 2011. Intelligent Automatic Generation Control, CRC Press, Boca Raton, FL.

Billinton, R. 1972. "Bibliography on the Application of Probability Methods in Power System Reliability Evaluation." IEEE Transactions on Power Apparatus and Systems, vol. PAS-91, pp. 649-660, 1972.

Billinton, R. and R.N. Allan. 1996. Reliability Evaluation of Power Systems, Plenum Press, NY.

Billinton, R., H. Chen and R. Ghajar. 1996. "A Sequential Simulation Technique for Adequacy Evaluation of Generating Systems Including Wind Energy." IEEE Transactions on Energy Conversion, vol. 11, no. 4, pp. 728-734, 1996.

Billinton, R. and A.A. Chowdhury. 1992. "Incorporating of Wind Energy Conversion Systems in Conventional Generating Capacity Adequacy Assessment." IEEE Proceedings C: Generation, Transmission and Distribution, vol. 139, no. 1, pp. 45-56, January 1992. Institute of Electrical and Electronics Engineers, Piscataway, NJ.

Billinton, R. and M. Fotuhi-Firuzabad. 1994. "A Basic Framework for Generating System Operating Health Analysis." IEEE Transactions on Power Systems, vol. 9, no. 3, pp. 1610-1617, August 1994.

Billinton, R. and B. Karki. 2011. "Well-Being Analysis of Wind Integrated Power Systems." IEEE Transactions on Power Systems, vol. 26, no. 4, November.

Billinton, R. and R. Karki. 1999. "Application of Monte Carlo Simulation to Generating System Well-Being Analysis." IEEE Transactions on Power Systems, vol. 14, no. 3, pp. 1172-1177, August 1999.

Billinton, R. and Khan. E. 1992. "A Security-Based Approach to Composite Power System Reliability Evaluation." IEEE Transactions on Power Systems, vol. 7, no. 1, pp. 936-941, February 1992.

Billinton, R., and W. Li. 1994. Reliability Assessment of Electric Power Systems Using Monte Carlo Methods. Plenum Press, New York 1994. 
Billinton, R. and Lian. G 1994. "Composite Power System Health Analysis Using a Security Constrained Adequacy Evaluation Procedure." IEEE Transactions on Power Systems, vol. 9, no. 2, pp. 936-941, May 1994.

BINE Informationsdienst, "Compressed Air Energy Storage Power Plants." BINE Informationsdienst projektinfo 05/07. Available http://www.bine.info/fileadmin/content/Publikationen/Englische Infos/projekt 0507 engl interne

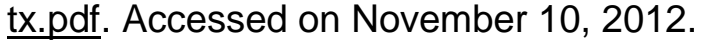

Bockenfeld, N. et al. 2011. "Composite LiFePO4/AC High-Rate Performance Electrodes for Liion Capacitors." J. Power Sources, 196: pp. 4136-4142.

Bolund, B. 2007. "Flywheel Energy and Power Storage Systems." Renewable and Sustainable Energy Reviews, vol. 11, pp. 235-259.

BPA. 2012. "BPA's Proposed Oversupply Management Protocol." BPA Workshop presentation, February 14, 2012. Available http://www.bpa.gov/Projects/Initiatives/Oversupply/OversupplyDocuments/20120207-proposedprotocol/Feb14WorkshopPresentation.pdf. Accessed November 27, 2012.

BPA. 2011. "Planning For and Responding to Overgeneration Events." BPA Public Workshop Presentation, February 25, 2011. [Online.] Available http://www.bpa.gov/Projects/Initiatives/Oversupply/OversupplyDocuments/Feb\%20Workshop\%2 OPresentations.pdf. Accessed November 27, 2012.

BPA. 2010a. "BPA Comments on RM10-11-000." Available http://www.bpa.gov/corporate/windpower/docs/RM10-11 BPA Comments.pdf. Accessed November 26, 2012.

BPA. 2010b. Forecast of Renewable Projects Connected to BPA Grid Based on Existing Queue and Recent Trends. Portland, OR. Available http://www.transmission.bpa.gov/PlanProj/Wind/documents/Wind Forecast Graph 2015September2010.pdf. Accessed October 30, 2012.

BPA. 2010c. "Comments of Bonneville Power Administration in FERC Docket No. RM10-11000." April 12, 2010. Available http://www.efsec.wa.gov/Whistling\%20Ridge/Adjudication/Intervenor's\%20prefiled\%20testimony/Ex\%2030.10\%20.pdf. Accessed November 12, 2012.

BPA. 2009. Integrating Wind Power and Other Renewable Resources into the Electric Grid, September 2009. Available http://bpa.gov/corporate/windpower/docs/WindWIT generic slide set Sep 2009 customer.pdf. Accessed November 26, 2012.

BPA. 2008a. "2009 Wind Integration Rate Case Revised Proposal." March 2008. Available http://www.nippc.org/upload/BPA\%20Wind\%20Integration\%20WI09_Revised_Proposal.pdf. Accessed November 26, 2012.

BPA. 2008b. "Proposed Wind Integration-Within-Hour Balancing Service Rate for Public Hearing, and Opportunity for Public Review and Comment." A Notice by the Bonneville Power Administration on February 7, 2008, Available https://www.federalregister.gov/articles/2008/02/07/E8-2253/proposed-wind-integration-within- 
hour-balancing-service-rate-for-public-hearing-and-opportunity-for. Accessed November 26, 2012.

BPA. Undated. "Bonneville Power Administration." Portland, OR. Available http://www.bpa.gov Accessed November 26, 2012.

Borges, C.L.T., and D. M. Falcao. 2000. "Power System Reliability by Sequential Monte Carlo Simulation on Multicomputer Platforms." In Proceedings of the Processing of the 4th International Conference Vector and Parallel (VECPAR 2000), Porto, Portugal, pp. 242-253, June 21-23, 2000.

Borkowska, B. 1974. "Probabilistic Load Flow." IEEE Transactions Power App. Systems, vol. PAS-93, pp. 752-759, May.

Bragason, R.F. 2005. Damping in the Icelandic Power System: Small Signal Stability Analysis and Solutions. MS Thesis, Dept. of Industrial Electrical Engineering and Automation, Lund University, Lund, Sweden, 2005. Available http://www.iea.Ith.se/publications/MS-

Theses/Full\%20document/5201 full document.pdf. Accessed November 27, 2012.

Brennan, B., M. Diedesch, J. Johnson, and W. Zhang. 2008. "Dynamic Line Ratings for the Cowlitz-LaGrande Transmission Lines." Final Report, Department of Electrical Engineering, Washington State University, Pullman, WA, 2008.

British Columbia Transmission Corporation. 2006. Voltage Stability Operating Limits And Procedures For Using The RT-VSA And VSA Applications, System Operating Order 7T - 50, Real Time Operations, British Columbia Transmission Corporation, Effective Date: May 2, 2006. Available http://www.bctc.com/NR/rdonlyres/BD53F0FD-A80A-4D0A-B4B911D13EFB9566/0/7T50.pdf. Accessed November 28, 2012.

Bucher,B. 2009. "King County Fuel Cell Demonstration Project." King County Department of Natural Resources and Park, Seattle, WA.

Buckley, P. 2010. "Ultra Capacitors with Increased Energy and Power Density Seek to Drive High-Power Applications." January. Available http://www.eetimes.com/electronicsnews/4198483/Ultracapacitors-with-increased-energy-and-power-density-seek-to-drive-highpower-applications?pageNumber=3. Accessed November 12, 2012.

Bullock, G.C. 1990. "Cascading Voltage Collapse in West Tennessee, August 22, 1987." Georgia Institute of Technology 44th Annual Protective Relaying Conference, May 2-4, 1990. Georgia Institute of Technology, Atlanta, GA.

Burke, A. 2007. "R\&D Considerations for the Performance and Application of Electrochemical Capacitors." Electrochem. Acta, 53:pp. 1083-1091.

Burke, K.A. 2003. Unitized Regenerative Fuel Cell System Development, N.G.R. Center, NASA/TM-2003-212739.

Butt, R. 2011. "Microgrid Planning and Design - MCAS Miramar, U.S. Air Force Academy, and Fort Carson." NREL, Golden, CO, Presentation May 13, 2011. Available http://e2s2.ndia.org/schedule/Documents/Abstracts/Butt.pdf. Accessed November 5, 2012. 
CAISO. 2011a. "Renewables Integration Market Vision and Roadmap, Initial Straw Proposal" California ISO, Folsom, CA. July 6, 2011. Available http://www.caiso.com/Documents/StrawProposalRenewablesIntegrationMarketVision RoadmapDay-ofMarket.pdf. Accessed November 27, 2012.

CAISO. 2011b. "Supplement to August 2010 Report on the Integration of Renewable Resources Operational Requirements and Generation Fleet Capability at 20\% RPS." Folsom, CA.

CAISO. 2010a. Integration of Renewable Resources: Operational Requirements and Generation Fleet Capability at 20\% RPS. California ISO Report, August 31, 2010. Available http://www.uwig.org/CAISO-20PercentRPS.pdf. Accessed November 27, 2012.

CAISO. 2010b. Interconnection Standards Review Initiative, Draft Straw Proposal, California ISO, Folsom, CA, March 25, 2010. Available http://www.nerc.com/docs/pc/ivgtf/CAISO_Draft_Straw_Proposal.pdf. Accessed November 15, 2012.

CAISO. 2010c. Interconnection Standards Review Initiative, Draft Straw Proposal, California ISO, Folsom, CA, May 7, 2010. Available http://www.caiso.com/Documents/SecondRevisedDraftFinalStrawProposalInterimInterconnectionRequirements_LargeGeneratorFacilitiesReview5_07_10.doc. Accessed November 15, 2012.

CAISO, 2009. "Participation of Limited Energy Storage Resources in CAISO Electricity Markets." Folsom, CA.

CAISO. 2006. "Spinning Reserve and Non-Spinning Reserve." California ISO, Folsom, CA, January.

CAISO 1998. California ISO System Disturbance Report: December 8, 1998 - San Francisco Area. CAISO, Folsom, CA. Available http://www.caiso.com/docs/1999/03/31/1999033116341717931.pdf.

Cañizares, C.A., and F.L. Alvarado. 1991. "Computational Experience with the Point of Collapse Method on Very Large AC/DC Systems." In Proceedings Bulk Power System Voltage Phenomena - Voltage Stability And Security, ECC/NSF Workshop, Deep Creek Lake, MD, August 1991; published by ECC Inc., Fairfax, VA.

Cañizares, C.A., F. L. Alvarado, C. L. DeMarco, I. Dobson, and W. F. Long, 1992. "Point of Collapse Methods Applied to AC/DC Power Systems." IEEE Transactions on Power Systems, vol. 7, no. 2, pp. 673-683, May 1992.

Cao, F., A. Charkey, and K. Williams. 2000. "Thermal Behavior and End-Of-Life Characteristics of the Nickel-Zinc Battery." In Proceedings of the 35th Intersociety Energy Conversion Engineering Conference, vol. 2, pp. 985-994.

Castellanos, R. and A.R. Messina. 2008. Robust Stability and Performance Analysis of Large Scale Power Systems with Parametric Uncertainty: A Structured Singular Value Approach, Nova Science Publishers, NY. 
Cavallo A. 2007. "Controllable and Affordable Utility-Scale Electricity from Intermittent Wind Resources and Compressed Air Energy Storage (CAES)." Energy 32:120-127.

Cavallo, A. 1995. "High-capacity Factor Wind Energy Systems." Journal of Solar Energy Engineering, vol. 117, no. 2, pp. 137-143.

"Central Allocation Office GmbH." Germany. Available http://www.central-ao.com. Accessed November 26, 2012.

Charkey, A, E. E. Ferreira and A. Charkey. 1997. "An Improved Nickel/Zinc Battery for Ventricular Assist Systems.” Journal of Power Sources, vol. 65, pp. 109-I 15.

Chauhan, S., and M.P. Dava. 1997. "Kohonen Neural Network Classifier for Voltage Collapse Margin Estimation." Electric Machines and Power Systems, vol. 25, no. 6, pp. 607-619, July.

Chen, G., S.R. Bare, and T.E. Mallouk. 2002. "Development of Supported Bifunctional Electrocatalysts for Unitized Regenerative Fuel Cells." Journal of The Electrochemical Society, 149(8):pp. A1092-A1099.

Chen, R.H., O. P. Malik, and G. S. Hope. 1995. "On-Line Multi-Contingency Preprocessing of Security Assessment for Severe Weather Based on Qualitative Reasoning with Probability Statistics Classification." Electrical Power and Energy Systems, vol. 17, pp. 3-10, 1995.

Chiang, H. D., F.F. Wu, and P.P. Varaiya, 1994. "A BCU Method for Direct Analysis of Power System Transient Stability." IEEE Transactions on Power Systems, vol. 9, no. 3, pp. 1194-1208.

China Daily. 2010. "State Grid Unveils Ultra-High-Voltage Power Line Plans." China Daily, August 13, 2010. Available http://www.chinadaily.com.cn/business/2010-08/13/content_11148692.htm. Accessed November 6, 2012.

Chmiola, J. et al. 2011. "Anomalous Increase in Carbon Capacitance at Pore Sizes Less Than 1 Nanometer." Science, 313:pp. 1760-1763.

CIGRE TB 299. 2006, Guide For the Selection of Weather Parameters for Bare Overhead Conductor Ratings. International Council on Large Electric Systems, Paris, France.

CIGRE Task Force 38. 1993. Modeling of Voltage Collapse Including Dynamic Phenomena. CIGRE Task Force 38-02-10. International Council on Large Electric Systems, Paris, France.

Clarke, S. et al. 2009. U.S. Offshore Wind Energy: A Path Forward. U.S. Offshore Wind Collaborative, Cambridge, MA. Available http://www.usowc.org/pdfs/PathForwardfinal.pdf. Accessed November 27, 2012.

Coates, D, E. Ferreira, and A. Charkey. 1997. "Development of a Long Cycle Life Sealed Nickel-Zinc Battery for High Energy-Density Applications." IEEE AES Systems Magazine, June.

ColumbiaGrid. 2008. Report on ACE Diversity Interchange. January 1, 2008. Available http://www.columbiagrid.org/download.cfm?DVID=436. Accessed February 2010. 
Conway, B.E., ed. 1999. Electrochemical Supercapacitors: Scientific Fundamentals and Technological Applications. Kluwer Academic/Plenum Publishers, Denmark.

Corey, G.P. 2010. An Assessment of the State of the Zinc-Bromine Battery Development Effort. RedFlow Limited, Chicago, IL.

Corey, G.P., L.E. Stoddard, and R.M. Kerschen. 2002. Boulder City Battery Energy Storage Feasibility Study. Sandia Report SAND2002-0751. Sandia National Laboratories, Albuquerque, NM.

Dalin, G.A., et al. 1964. "Sealed Silver-Zinc Batteries." In Proceedings of the 18th Annual Power Sources Conference. Fort Monmouth, NJ. p. 54. PSC Publications Committee, Red Bank, NJ.

Daniel, R, 2010. "Integrating Wind-Solar-CAES," Compressed Air Energy Storage (CAES) Conference \& Workshop, October 21, 2010. Available http://www.clca.columbia.edu/CAES2workshop_proceedings.pdf.

Desrochers, G., and M. Blanchard. 1986. "A Monte Carlo Simulation for the Economical Assessment of the Contribution of Wind Energy to Power Systems," IEEE Transactions on Energy Conversion, vol. 1, no.4, pp. 50-56, 1986.

Dimo, P. 1975. Nodal Analysis of Power Systems. Abacus Press, England.

Dimo, P. 1971. L’Analyse Nodale des Réseaux D'Énergie, Eyrolles.

Dinavahi, V.R., and S.C. Srivastava. 2001. "ANN-Based Voltage Stability Margin Prediction." IEEE Power Engineering Society Summer Meeting, vol. 2, pp. 1275-1280, July 15-19, 2001. Institute of Electrical and Electronics Engineers, Piscataway, NJ.

Dizdarevic, N., M. Majstrovic, S. Cudjic Coko, N. Mandic, and J. Bonovic. 2003. "Causes, Analyses and Countermeasures with Respect to Blackout in Croatia on January 12, 2003." CRIS International Workshop on Power System Blackouts Causes, Analyses, and Countermeasures, Lund, Sweden, May 3, 2004.

Djukanovic, M., D.J. Sobajic and Y.H. Pao. 1993. "Learning Tangent Hypersurfaces for Fast Assessment of Transient Stability" In Proceedings of 2nd International Forum on Applications of Neural Networks to Power Syst. ANNPS '93, April 19-22, 1993, pp. 124-129. Institute of Electrical and Electronics Engineers, Piscataway, NJ.

Dobson, I. 1992. "Observations on the Geometry of Saddle Node Bifurcation and Voltage Collapse in Electrical Power Systems." IEEE Transactions on Circuits and Systems I: Fundamental Theory and Applications, vol. 39, no. 3, pp. 240-243, March 1992.

Dobson, I. and L. Lu. 1993. "New Methods for Computing a Closest Saddle Node Bifurcations and Worst Case Load Power Margin for Voltage Collapse." IEEE Transactions on Power Systems, vol. 8, no. 3, pp. 905-913, August.

Dobson, I., and L. Lu. 1992. "Computing an Optimal Direction in Control Space to Avoid Saddle Node Bifurcation and Voltage Collapse in Electric Power Systems." IEEE Transactions on Automatic Control, vol. 37, no. 10, pp. 1616-1620, October. 
Dobson, I., L. Lu and Y. Hu. 1991. "A Direct Method for Computing a Closest Saddle Node Bifurcation in the Load Power Parameter Space of an Electric Power System." IEEE International Symposium on Circuits and Systems, Singapore, pp. 3019-3022, June. Institute of Electrical and Electronics Engineers, Piscataway, NJ.

DOE. 2011. "One Million Electric Vehicles By 2015.” DOE, Washington, D.C. editor.

DOE. 2003. Operations Review of June 14, 2000 PG\&E Bay Area System Events Using AEMPFAST ${ }^{\circledR}$ Software. Consultant Report P500-03-085F, Prepared by CERTS for the California Energy Commission, Folsom, CA, October.

Dolloff, P.A., D. Adams, S. Anderson, M.Y. Vaiman, M.M. Vaiman, M. Povolotskiy, and D.T. Bradshaw. 2008. "Evaluate Real-time System Reliability Using Boundary-based Concept for EKPC System." In Proceedings of 2008 PES General Meeting, July 20-24, 2008. Institute of Electrical and Electronics Engineers, Piscataway, NJ.

Dong, Z.Y., Y.V. Makarov, and D. J. Hill. 1998. "Power System Small Signal Stability Analysis Using Genetic Optimization Techniques." Electric Power Systems Research, vol. 46, 1998, pp. 195-204.

Dong, C. and Y, Yu. 2005. "PDSR in Phase Angle Space and SPM Based Security Monitoring of Power Systems." IEEE Region 10 TEHCON 2005, pp. 1-5, November 2005. Institute of Electrical and Electronics Engineers, Piscataway, NJ.

Doorman, G., G. Kjǿlle, K. Uhlen, E. S. Huse, and N. Flatabǿ. 2004.Vulnerability of the Nordic Power System. Report to the Nordic Council of Ministers, SINTEF Energy Research, May 2004. Available http://sparky.harvard.edu/hepg/Papers/Doorman.vul.nordic. system.0504.pdf Accessed November 12, 2012.

Dopazo, J.F. 1975. "Stochastic Load Flow." IEEE Transactions Power Apparatus and Systems, vol. PAS-94, pp. 299-309, March.

Douglas, D. 2002. High-Temperature, Low-Sag Transmission Conductors. Electric Power Research Institute, Palo, Alto, CA. Available http://mydocs.epri.com/docs/public/000000000001001811.pdf. Accessed November 7, 2012.

Drury, E. P. Denholm, and R. Sioshans. 2011. "The Value of Compressed Air Energy Storage in Energy and Reserve Markets." Energy 36:4959-4973.

Dvorak, P. 2011. "High Voltage DC Line Planned to Cross lowa." Windpower Engineering and Development, June 13, 2011. Available http://www.windpowerengineering.com/featured/business-news-projects/high-voltage-dc-lineplanned-to-cross-iowal. Accessed November 15, 2012.

EASAC, Transforming Europe's Electricity Supply - An Infrastructure Strategy for a Reliable, Renewable and Secure Power System. EASAC Policy Report 11, The Royal Society, London. Available http://easac.eu/fileadmin/PDF_s/reports_statements/Transforming.pdf.

East Penn Manufacturing Co. Inc. 2010. "East Penn Awarded Grant from the DOE for UltraBattery Smart Grid Project." February. Available http://www.dekabatteries.com/default.aspx?pageid=1813. Accessed November 12, 2012. 
Eckroad, S. 2007. "Vanadium Redox Flow Batteries: An In-Depth Analysis." Rept \# 1014836. Electric Power Research Institute, Palo Alto, CA.

Eckroad, S. 2004. "EPRI-DOE Handbook Supplement of Energy Storage for Grid Connected Wind Generation Applications." Rept \# 1008703. Electric Power Research Institute. Palo Alto, CA, and the U.S. Department of Energy, Washington, D.C.

Edris, A-A., R. Adapa, M. H. Baker, L. Bohmann, K. Clark, K. Habshi, L. Gyugyi, J. Lemay, A.S. Mehraban, A. K. Myers, J. Reeve, F. Sener, D. R. Torgerson, and R. R. Wood. 1997. "Proposed Terms and Definitions for Flexible AC Transmission System (FACTS)." IEEE Transactions on Power Delivery, vol. 12, pp. 1848-1853, October. Institute of Electrical and Electrics Engineers, Piscataway, NJ.

El-Hawary, M.E. 2007. Power System Stability and Control. CRC Press, Baco Raton, FL.

El-Hawary, M.E., and G.A.N. Mbamalu. 1991. "A Comparison of Probabilistic Perturbation and Deterministic Based Optimal Power Flow Solutions." IEEE Transactions in Power Systems, vol. 6, no. 3, pp. 1099-1105, August.

El-Kady, M.A., C. K. Tang, V. F. Carvalho, A. A. Fouad, and V. Vittal. 1986. "Dynamic Security Assessment Utilizing the Transient Energy Function Method." IEEE Trans. on Power Syst., 1(3), pp. 284-291, 1986.

El-Keib A.A., and X. Ma. 1995. "Application of Artificial Neural Networks in Voltage Stability Assessment." IEEE Transactions on Power Systems, vol. 10, no. 4, pp. 1890-1896, November.

El-Sharkawi, M.A., R.J. Marks, M.E. Aggoune, D.C. Park, M.J. Damborg, and L.E. Atlas. 1989. "Dynamic Security Assessment of Power Systems Using Back Error Propagation Artificial Neuron Networks." In Proceedings of the 2nd Symposium on Expert System Applications to Power Systems, Seattle, WA, July 1989.

Ela, E. and B. Kirby. 2008. ERCOT Event on February 26, 2008: Lessons Learned. Report, NREL/TP-500-43373, National Renewable Energy Laboratory, Golden, CO, July 2008. Available www.nrel.gov/docs/fy08osti/43373.pdf. Accessed November 15, 2012.

Ela, E., B. Kirby, E. Lannoye, M. Milligan, D. Flynn, B. Zavadil, and Mark O'Malley. 2010. "Evolution of Operating Reserve Determination in Wind Power Integration Studies." IEEE Power and Energy Society General Meeting, July 25-29, 2010, Minneapolis, MN, pp. 1-8. Institute of Electrical and Electronics Engineers, Piscataway, NJ. Available http://www.wecc.biz/committees/StandingCommittees/JGC/VGS/OWG/ActivityO1/Operating\%2 OReserve\%20Determination\%20in\%20Wind\%20Integration\%20Studies.pdf. Accessed November 20, 2012.

Elkraft-System. 2003. Power Failure in Eastern Denmark and Southern Sweden on 23 September 2003. Final report on the course of events, Elkraft System, DENMARK. November 4, 2003. Available www.geocities.jp/ps dictionary/blackout/Final report uk-web.pdf.

Ellis, A., E. Muljadi, J.J. Sanchez-Gasca, and Y. Kazachkov. 2011. "Generic Models for Simulation of Wind Power Plants in Bulk System Planning Studies." In Proceedings of 2011 
IEEE Power and Energy Society General Meeting, July 24-29, 2011, San Diego, CA, pp. 1-8. Institute of Electrical and Electronics Engineers, Piscataway, NJ.

Energizer Resources. 2011. Vanadiumsite, Vancouver, B.C. Available http://www.vanadiumsite.com/vanadium-redox-l-cell-iron-chromium-flow-batteryl. Accessed October 3, 2011.

Energy Storage News, 2010. Available http://www.energystoragenews.com/Vanadium\%20Redox\%20Flow\%20Batteries.htm. Accessed October 3, 2011.

EPRI. 1993. Voltage Stability/Security Assessment and On-Line Control. EPRI Technical Report TR-101931-V1-4, vol. 1-4,. Electric Power Research Institute, Palo Alto, CA..

EPRI. 1992. Voltage Stability Analysis Program (VSTAB), Version 2.1, User's Manual. EPRI Research Project RP30400-01, August 1992. Electric Power Research Institute, Palo Alto, CA.

ERCOT, "Glossary." ELECTRIC Reliability Council of Texas (ERCOT), Austin, TX. Available http://www.ercot.com/glossary. Accessed November 28, 2012.

ERCOT. 2010. "The Tres Amigas Project." ERCOT Presentation, Tres Amigas L.L.C., Santa Fe, NM . Available

http://www.google.com/url?sa=t\&rct=i\&q=the\%20tres\%20amigas\%20project $\% 2$ C $\% 20$ ercot $\% 20$ presentation\&source=web\&cd=1\&cad=rja\&ved=0CDAQFjAA\&url=http\%3A\%2F\%2Fwww.ercot. com\%2Fcontent\%2Fmeetings\%2Frpg\%2Fkeydocs\%2F2010\%2F0122\%2FTA ERCOT FINAL 012110.ppt\&ei=EgrAUIn7NcbL0AGj04BY\&usg=AFQjCNH7Okcs42JiJlhz1BQwLCAZZFKOjQ

Accessed December 5, 2012.

Ernst, B., U. Schreirer, F. Berster, C. Scholz, H.-P. Erbring, S. Schlunke, J. Pease, and Y. V. Makarov. 2010. Large Scale Wind and Solar Integration in Germany. PNNL-19225, Pacific Northwest National Laboratory, Richland, WA. Available:

http://www.pnl.gov/main/publications/external/technical reports/PNNL-19225.pdf. Accessed November 15, 2012.

Eto, J.H., J. Undrill, P. Mackin, R. Daschmans, B. Williams, B. Haney, R. Hunt, J. Ellis, H. Illian, C. Martinez, M.O'Malley, K. Coughlin, and K. Hamachi LaCommare. 2010. Use of Frequency Response Metrics to Assess the Planning and Operating Requirements for Reliable Integration of Variable Renewable Generation. Lawrence Berkeley National Laboratory, Berkeley, CA. December 2010.

Eudy, L. 2011. "Technology Validation: Fuel Cell Bus Evaluations," in DOE Hydrogen and Fuel Cells Program Annual Progress Report. DOE, Washington, D.C. Editor. Intelligent Energy, Long Beach, CA. pp. 1036-1039.

European Commission. 2003, "Report on the Application of the Regulation (EC) No 1228/2003 of the European Parliament and of the Council," June 2003. Available http://www.energycommunity.org/pls/portal/docs/36276.PDF.

"European Network of Transmission System Operators for Electricity." ENTSO-E Website: Available http://www.entsoe.eu/. Accessed November 26, 2012. 
European Wind Energy Association. 2010. Powering Europe: wind energy and the electricity grid. November 2010. Available

http://www.ewea.org/fileadmin/ewea documents/documents/publications/reports/Grids Report 2010.pdf. Accessed November 10, 2012.

Evdokunin, G.A. 2004. "Efficiency of the Use of Power Transmission with Increased SurgeImpedance Loading (ISIL)." Expanding Edge Seminar, Midwest ISO, St. Paul, MN, September 16, 2004. Available http://www.cerc-

reactors.com/articles/miso16sep2004seminar/3 Evdokunin/Evdokunin-Presentation.pdf.

Accessed October 31, 2012.

Eyer, J., and G. Corey. 2010. Energy Storage for the Electricity Grid: Benefits and Market Potential Assessment Guide. Sandia National Laboratory, Albuquerque, NM.

Eyer, J. and R.S. Brown. 2007. Guide To Estimating Benefits and Market Potential for Electricity Storage in New York (With Emphasis on New York City). Final Report 07-06, New York State Energy Research and Development Authority (NYSERDA), Albany, NY. March 2007. Available http://www.energycollection.us/Energy-Storage/NYSERDA-Storage-Benefits.pdf. Accessed November 27, 2012.

Eyer, J., and G. Corey. 2010. Energy Storage for the Electricity Grid: Benefits and Market Potential Assessment Guide. Sandia National Laboratory, Albuquerque, NM.

FACTS Terms \& Definitions Task Force. 1997. "Proposed Terms and Definitions for Flexible AC Transmission System (FACTS)." IEEE Transactions on Power Delivery, vol.12, no.4, pp.18481853, October 1997. Institute of Electrical and Electronics Engineers, Piscataway, NJ.

FERC. 2012a. "Order No. 1000 - Transmission Planning and Cost Allocation." FERC, Washington, D.C. Jan 2012 Available http://www.ferc.gov/industries/electric/indus-act/transplan.asp. Accessed November 28, 2012.

FERC. 2012b. "Federal Energy Regulatory Authority (FERC) Order 1000." May 17, 2012. Transmission Planning and Cost Allocation by Transmission Owning and Operating Public Utilities. FERC, Washington, D.C. Available http://www.ferc.gov/whats-new/commmeet/2012/051712/E-1.pdf. Accessed November 6, 2012.

FERC 2010a. National Action Plan on Demand Response. Federal Energy Regulatory Commission, Washington, D.C. June 2010. Available http://www.ferc.gov/legal/staff-reports/0617-10-demand-response.pdf. Accessed November 20, 2012.

FERC 2010b. Demand Response Compensation in Organized Wholesale Energy Markets. FERC Notice of Proposed Rulemaking, issued March 18, 2010. FERC, Washington, D.C.

FERC 2009. A National Assessment of Demand Response Potential. Federal Energy Regulatory Commission, Staff Report, Washington, D.C. June 2009, p. 27. Available http://www.ferc.gov/legal/staff-reports/06-09-demand-response.pdf. Accessed November 20, 2012 .

FERC. 2005. FERC Order No. 661-A. December 2005. Available http://www.ferc.gov/EventCalendar/Files/20051212171744-RM05-4-001.pdf. Accessed 11/8/12. 
FERC 2008 Wholesale Competition in Regions with Organized Electric Markets. FERC Order No. 719. FERC, Washington, D.C. Available http://www.ferc.gov/whats-new/commmeet/2008/101608/E-1.pdf.

Forbes, B., D. Bradshaw, and F. Campbell. 2002. "Finding Hidden Capacity In Transmission Lines." Transmission and Distribution World, September 1, 2002.

Fouad, A.A. and V. Vittal. 1992. Power System Transient Stability Analysis Using the Transient Energy Function Method. Prentice Hall, NJ, 1992.

Freeman, Sullivan \& Co, Energy and Environmental Economics, Inc, 2009, California Independent System Operator Demand Response Barriers Study. April 2009. Available http://www.caiso.com/2410/2410ca792b070.pdf.

Furukawa, J. et al. 2009. "Development of the Flooded-type UltraBattery for Micro-HEV Applications." ECS Transactions, 16(34):pp. 27-34.

Galloway, R,C. 2003. "ZEBRA Battery - Material Cost Availability and Recycling." Published EVS-20. Long Beach, CA.

Gao, B., G.K. Morison, and P. Kundur. 1996. "Towards the Development of a Systematic Approach for Voltage Stability Assessment of Large-Scale Power Systems." IEEE Transactions on Power Systems, vol. PWRS-11, no. 3, pp. 1314-1324, August 1996. Institute of Electrical and Electronics Engineers, Piscataway, NJ.

Garver, L.L. 1966. "Effective Load Carrying Capability of Generating Units." IEEE Transactions on Power Appuratus and Systems, vol. 85, no. 8, pp. 910-919, August.

Gautam, D., V. Vittal, and T. Harbour. 2009. "Impact of Increased Penetration of DFIG-Based Wind Turbine Generators on Transient and Small Signal Stability of Power Systems." IEEE Transactions on Power Systems, vol. 24, no. 3, pp. 1426-1434, August.

Gestore Rete Transmissione 2003. Black-Out: The Events of 28 September 2003. Press Release, Gestore Rete Transmissione Nazionale, Italy, October 1, 2003. Available http://www.grtn.it/eng/documentinewsstatiche/blackout28set03.pdf.

Giberson, M. 2009. "Consequences of Dynamic Transmission Line Rating for Renewable Power." Blog, May 20, 2009, Available http://knowledgeproblem.com/2009/05/20/dynamictransmission-line-rating-and-renewable-powerl. Accessed October 31, 2012.

Global Transmission 2009. "Focus on UHV AC: China Shows the Way by Energizing 1,000 kV Line." Global Transmission Report, New Delhi, India. Available http://www.globaltransmission.info/archive.php?id=1434. Accessed November 20, 2012.

Golden Valley Electric Association. "Battery Energy Storage System (BESS)." Golden Valley Electric Association, Fairbanks, AK. Available http://www.gvea.com/inside/about. Accessed November 20, 2012.

Gray, B. 2009. "Grid Scale Energy Storage: Linchpin Technology for Our Clean and Secure Energy Future." Environmental Energy Technologies Division Seminars, E.O. Lawrence 
Berkeley National Laboratory, Berkeley, CA. March 17, 2009. Available http://eetdseminars.lbl.gov/seminar/grid-scale-energy-storage-linchpin-technology-our-clean-and-secureenergy-future. Accessed November 30, 2011.

Grazzini, G. and A. Millazzo. 2008. "Thermodynamic Analysis of CAES/TES Systems for Renewable Energy Plants." Renewable Energy 44:1998-2006.

Green Economy. 2011. "First HVDC Transmission Projects Beginning Globally." Green Economy, May 2, 2011. Available http://uk.ibtimes.com/articles/20110502/first-hvdctransmission-projects-beginning-globally.htm. Accessed November 15, 2012.

Greenblatt, J.B. 2005. "Using Gas Turbines to Enhance the Value of Wind Power." Combined Cycle J 1Q:34-8, 2005.

Greene, S., I. Dobson, and F.L. Alvarado. 2002. "Sensitivity of Transfer Capability Margins with a Fast Formula." IEEE Transactions on Power Systems, vol. 17, issue 1, February 2002, pp. 3440.

Greene, S., I. Dobson, and F.L. Alvarado. 1997. "Sensitivity of the Loading Margin to Voltage Collapse with Respect to Arbitrary Parameters." IEEE Transactions on Power Systems, vol. 12, issue 1, February 1997, pp. 262-272.

Grigoriev, S.A. et al. 2010. "Design and Characterization of Bi-Functional Electrocatalytic Layers for Application in Pem Unitized Regenerative Fuel Cells." International Journal Hydrogen Energy, 35:p. 5070-5076.

Group, N. 2010. "Advanced Materials and Devices for Stationary Electrical Energy Storage Applications." DOE, Washington, D.C., editor. p. 25.

Grünbaum, R. 2010. "FACTS for Grid Integration of Wind Power." ABB, Montreal, Canada. Available http://www05.abb.com/global/scot/scot221.nsf/veritydisplay/0bb9837cbc3c970e832577bb003e4 464/\$file/IEEE\%20PES\%20ISGT\%202010.pdf. Accessed November 20, 2012.

Gurevich, Y. E., L.E. Libova, and A.A. Okin. 1990. Design of Stability and Emergency Control Automatic Devices in Power Systems. Energoatomizdat, Moscow, 1990 - in Russian.

Gyuk, I.P. and S. Eckroad. 2003. "EPRI-DOE Handbook of Energy Storage for Transmission and Distribution Applications." Rept \# 1001834 Final Report, December. Prepared by Technology Insights and EPRI PEAC Corporation for the U.S. Department of Energy, Washington D.C., and Electric Power Research Institute, Inc., Palo Alto, CA.

Hagstrom, E., I. Norheim, and K. Uhlen. 2005. "Large-Scale Wind Power Integration in Norway and Impact on Damping in the Nordic Grid." Wind Energy, vol. 8, no. 3, pp. 375-384.

Hailei, H., G. Jianbo, S. Yunting, and Z. Dongxia, "Risk-Based Probabilistic Security Evaluation Algorithm of Bulk Power System." In Proceedings of the 3rd International Conference on Electric Utility Deregulation and Restructuring and Power Technologies, pp. 1231-1236. Institute of Electrical and Electronics Engineers, Piscataway, NJ. 
Hakim, H. 2002. "Application of Pattern Recognition in Transient Security Assessment." Journal of Electrical Machines and Power Systems, vol. 20, no. 1-15.

Han, C., A.Q. Huang, M.E. Baran, S. Bhattacharya, W. Litzenberger, L. Anderson, A.L. Johnson, and A.-A. Edris. 2008. "STATCOM Impact Study on the Integration of a Large Wind Farm into a Weak Loop Power System." IEEE Transaction on Energy Conversion, March 2008. Institute of Electrical and Electronics Engineers, Piscataway, NJ.

Hansen, A.D., F. Iov, P. Sorensen, N. Cutuluis et al. 2007. "Dynamic Wind Turbine Models in Power System Simulation Tool DIgSILENT." Ris0-R-1400(EN).

Hardiman, R.C., M. Kumbale, and Y. V. Makarov. 2004. "An Advanced Tool for Analyzing Multiple Cascading Failures." In Proceedings of the 8th International Conference on Probabilistic Methods Applied to Power Systems, Iowa State University, Ames, Iowa, September 12-16, 2004.

Harris, C. and J.P. Meyers. 2010. "Working Smarter, Not Harder: An Introduction to the Smart Grid." The Electrochemical Society Interface, 19(3).

Harris, P.G. 2010. "Technology Attributes.” Fortnightly's Spark, pp. 2-10.

Hassenzahl, W.V. Eckroad, S. E. C. Grant. P. M. Gregory, B. and Nilsson, S.2009. "A HighPower Superconducting DC Cable," IEEE Transactions on Applied Superconductivity, vol. 19, no. 3, June 2009, pp. 1756-1761.

Hatziargyriou, N.D., T. S. Karakatsanis, and M. Papadopoulos. 1993. "Probabilistic Load Flow in Distribution Systems Containing Dispersed Wind Power Generation." IEEE Transactions on Power Systems, vol. PWRS-8, pp. 159-165, February.

Herman, D. 2003. "Comparison of Storage Technologies for Distributed Resource Applications." Rept \#1007301, Electric Power Research Institute, Palo Alto, CA.

Heydt, G.T., V. Vittal, S. Malhara, Y. V. Makarov, N. Zhou, and P. V. Etingov. 2011. "The Characterization and Impact of Extreme Forecast Errors on Power Systems." Journal of Electric Power Components and Systems, vol. 39, no. 15, pp. 1685-1700, October 2011.

Himy, A. 1986. Silver-Zinc Battery, Phenomena and Design Principles. Vantage Press, Inc., Burlington, VT.

Hingorani, N.G. 1988. "Power Electronics in Electric Utilities: Role of Power Electronics in Future Power Systems." Invited Paper, Proceedings of the IEEE Special Issue, vol. 76, no. 4, April 1988. Institute of Electrical and Electronics Engineers, Piscataway, NJ.

Hingorani, N.G., and L. Gyugyi. 2000. "'Understanding FACTS: Concepts and Technology of Flexible AC Transmission Systems." IEEE Press, NY.

Hiskens, I.A. and R. J. Davy. 2001. "Exploring the Power Flow Solution Space Boundary." IEEE Power Engineering Review, vol. 21, no. 8, August 2001, pp. 57-57. 
Hoffman P., P, H. Miller, and J. Kerth, 2008. "McIntosh CAES Experience NYSERDA-Columbia University Compressed Air Energy Workshop," Compressed Air Energy Storage (CAES)

Conference \& Workshop, Columbia University, October 21-22, 2008. Columbia University, NY.

Holbert, K. E. and G. T. Heydt. 2001. "Prospects For Dynamic Transmission Circuit Rating." Proceedings of IEEE International Symposium on Circuits and Systems (ISCAS), Sydney, Australia, vol. 3, pp. 205-208, May 2001. Institute of Electrical and Electronics Engineers, Piscataway, NJ. Available http://www.pserc.wisc.edu/documents/publications/papers/2001 general publications/heydtsydney01-4.pdf. Accessed November 7, 2012.

Holman, J. 2011. "Increasing transmission capacity," Wind Systems. Available windsystemsmag.com, pp. 50-53, January.

Holttinen, H., P. Meibom, A. Orths, F. Van Hulle, B. Lange, M. O'Malley, J. Pierik, B. Ummels, J.O. Tande, A. Estanqueiro, M. Matos, E. Gomez, L. Söder, G. Strbac, A. Shakoor, J. Ricardo, J.C. Smith, M. Milligan, and E. Ela. 2009, Design and Operation of Power Systems with Large Amounts of Wind Power. Final Report IEA Wind Task 25 (2006-2008) ISBN 978-951-38-7308-0 VTT, Vuorimiehentie. Available http://www.vtt.fi/inf/pdf/tiedotteet/2009/T2493.pdf.

Hou, J. et al. 2011. "Graphene-based Electrochemical Energy Conversion and Storage: Fuel Cells, Supercapacitors and Lithium Ion Batteries." Phys. Chem. Chem. Phys., vol. 13, pp. 15384-15402.

Huang, D. Yinbiao Shu, Jiangjun Ruan, and Yi Hu. 2009. "Ultra-High Voltage Transmission in China: Developments, Current Status and Future Prospects." In Proceedings of the IEEE, vol.97, no.3, pp.555-583, March 2009. Institute of Electrical and Electronics Engineers, Piscataway, NJ.

Huang, H., V. Ramaswami, and D. Kumar. 2002. "Design Considerations of Ultra High Voltage DC System." December 2002. Available http://www.ptd.siemens.de/050714 Design Considerations UHVDC.pdf. Accessed October 31, 2012.

Huggins, R.A. ed. 2010. Energy Storage. Springer, U.S., 2010

Hughes, F.M., O. Anaya-Lara, N. Jenkins, et al. 2006. "A Power System Stabilizer for DFIGBased Wind Generation." IEEE Transactions on Power Systems, vol. 21, no. 2, pp. 763-772, May.

Hyungchul, K., and C. Singh. 2002. "Probabilistic Security Analysis Using SOM and Monte Carlo Simulation." In Proceedings of IEEE Power Engineering Society Winter Meeting, vol.2, pp. 755-760, 2002. Institute of Electrical and Electronics Engineers, Piscataway, NJ.

Hydrogen Analysis Resource Center Website,_Available http://hydrogen.pnl.gov/cocoon/morf/hydrogen/site specific/fuel heating calculator?canprint=fal se. Accessed November 28, 2012.

IEA Wind. 2011. "IEA Task 25, Power Systems with Large Amounts of Wind Power." Available http://www.ieawind.org/summary page 25.html. Accessed November 26, 2012. 
IEEE. 2006. "IEEE Standard 738-2006, IEEE Standard for Calculating the Current-Temperature of Bare Overhead Conductors," January 30, 2006. Institute of Electrical and Electronics Engineers, Piscataway, NJ.

IEEE. 1990. Voltage Stability of Power Systems: Concepts, Analytical Tools, and Industry Experience. IEEE Special Publication 90TH0358-2-PWR, Institute of Electrical and Electronics Engineers, Piscataway, NJ.

IEEE Excitation Limiters Task Force. 1995. "Recommended Models for Overexcitation Limiting Devices." IEEE Transactions on Energy Conversion, vol. 10. no. 4, pp. 706-712, December.

IEEE Standards Association. 2011. "IEEE Guide for Design, Operation, and Integration of Distributed Resource Island Systems with Electric Power Systems." Institute of Electrical and Electronics Engineers, Piscataway, NJ. July, 2011. Available http://ieeexplore.ieee.org/stamp/stamp.jsp?tp=\&arnumber=5960751. Accessed December 2, 2012.

IEEE Standard Dictionary of Electrical \& Electronics Terms (ANS/IEEE Std 100-1977). 1997. IEEE Press, New York. Available http://ieeexplore.ieee.org/xpl/mostRecentlssue.jsp?punumber=4116785. Accessed November 28, 2012.

International Council on Large Electric Systems - CIGRE (HVDC and Power Electronics) Committee. Undated. "Overview of Flexible AC Transmission Systems, Facts."Available http://www.google.com/url?sa=t\&rct=i\&q=\&esrc=s\&frm=1\&source=web\&cd=1\&ved=0CDAQFjA A\&url=http\%3A\%2F\%2Fb4.cigre.org\%2Fcontent\%2Fdownload\%2F1973\%2F25265\%2Fversion \%2F2\%2Ffile\%2FFACTS\%2Boverview Cigr\%25C3\%25A9\%2BB4 What\%2Bis\%2BFACTSID1 OVER39.pdf\&ei=cDGcUJaHDMvqiwKLgoGYAQ\&usg=AFQjCNHv8TfBNxEet81m4Q8nF5oJh69 43A\&sig2=T4SP jhylZNW2GUCunx-1w. Accessed November 20, 2012.

Intrator, J. et al. 2011. 2020 Strategic Analysis of Energy Storage in California. Public Interest Energy Research (PIER) Program, California Energy Commission, University of California, Berkeley, CA.

Irisarri, G.D., X. Wang, J. Tong, and S. Mokhtari. 1997. "Maximum Loadability of Power Systems Using Interior Point Non-linear Optimization Method." IEEE Transactions on Power Systems, vol. 12, no. 1, pp. 162-172, February 1997.

Irving, P. 2011. "Power Generation from an Integrated Biomass Reformer and Solid Oxide Fuel Cell." In DOE Hydrogen and Fuel Cells Program Annual Progress Report, DOE, editor, Washington, D.C. InnovaTek Inc., Richland, WA, pp. 901-904.

ISO/RTO Council Planning Committee. 2006. Phase I Transmission Planning Report. March 20, 2006. Available http://www.iso-ne.com/pubs/spcl rpts/2006/irc pc planning report.pdf. Accessed November 5, 2012.

Itron. 2010. Impacts of Distributed Generation. Prepared for California Public Utilities Commission by Itron Inc., Davis, CA, January 2010, pp. 3-7. Available www.cpuc.ca.gov/NR/rdonlyres/750FD78D-9E2B-4837-A81A6146A994CD62/0/ImpactsofDistributedGenerationReport 2010.pdf. Accessed November 27, 2012. 
Jarjis, J. and F.D. Galiana. 1981. "Quantitative Analysis of Steady State Stability in Power Networks." IEEE Transactions on Power Apparatus and Systems, vol. PAS-100, no. 1, pp. 318326, January 1981.

Joerissen, L., J. Garche, Ch Fabjan, and G. Tomazic. 2004. "Possible Use of Vanadium RedoxFlow Batteries for Energy Storage in Small Grids and Stand-Alone Photovoltaic Systems." J. Power Sources, vol. 127, pp. 98-104.

Johnson, R.C. 2011. "Gasoline, Diesel or Pumpable Electrons." Electronic Engineering Times. 2011, pp. 22-28.

Jossen, A., and D.U. Sauer. 2006. "Advances in Redox-Flow Batteries." First International Renewable Energy Storage Conference, October 30-31, 2006, Gelsenkirchen, Germany.

Kaizuka, T. and T. Sasaki. 2001. "Evaluation of Control Maintaining Electric Power Quality By Use of Rechargeable Battery System." Power Engineering Society Winter Meeting, pp. 88-93 vol.1. Institute of Electrical and Electronics Engineers, Piscataway, NJ.

Kalsi, S., D. Madura, R. Howard, G. Snitcbler, T. MacDonald, D. Bradshaw. I. Grant, and M. Ingram. 2003. "Superconducting Dynamic Synchronous Condenser for Improved Grid Voltage Support." 2003 IEEE T\&D Conference, Dallas, Texas. Institute of Electrical and Electronics Engineers, Piscataway, NJ. September 2003.

Karpinski, A,,et al. 1999. "Silver-Zinc: Status of Technology and Applications," Journal of Power Sources 80, pp. 53-60.

Karystianos, M.E., N. G. Maratos, and C. D. Vournas. 2007. "Maximizing Power-System Loadability in the Presence of Multiple Binding Complementarity Constraints." IEEE Transactions on Circuits and Systems I: Regular Papers, vol. 54, no. 8, pp: 1775-1787.

Kataoka, Y. and Y. Shinoda. 2005. "Voltage Stability Limit of Electric Power Systems with Generator Reactive Power Constraints Considered." IEEE Transactions on Power Systems, vol. 20, no. 2, pp. 951-962, May 2005.

Kaye, R. and F. Wu. 1982. "Dynamic Security Regions of Power Systems." IEEE Transactions on Circuits and Systems, vol. 29, no. 9, September 1982, pp. 612-623.

Kazaryan, S.A., S.N. Razumov, and S.V. Litvinenko. 2006. "Mathematical Model of Heterogeneous Electrochemical Capacitors and Calculation of Their Parameters," Journal of The Electrochemical Society, vol. 153, p. A1655.

Keane, A., M. R. Milligan, C. J. Dent, B. Hasche, C. D'Annunzio, K. Dragoon, H. Holttinen, N. Samaan, L. S“oder, and M. O'Malley. 2011. "Capacity Value of Wind Power." IEEE Transactions on Power Systems, vol. 26, pp. 564-572, May.

Kear, G., A.A. Shah, and F.C .Walsh. 2011. "Development of the All- Vanadium Redox Flow Battery for Energy Storage: A Review of Technological, Financial and Policy Aspects." International Journal of Energy Research 2011, DOI: 10.1002/er.1863. 
Kim, J.H. et al. 2011. "Effect of Carbon Types on the Electrochemical Properties of Negative Electrodes for Li-Ion Capacitors." J. Power Sources, 196:pp. 10490-10495.

Kirby, B.J. 2003. "Spinning Reserve From Responsive Loads." Oak Ridge National Laboratory, Oak Ridge, TN. March 2003.

Kontorovich, A.M., A.V. Kryukov, Y.V. Makarov, et al. 1988. Methods of Stability Indices Computations for Complicated Power Systems. Publishing House of the Irkutsk University, Irkutsk, 1988 - in Russian.

Kontorovich, A.M. and Y. V. Makarov. 1989. "A Permanent Loading Technique for Fast Analysis of Power System Stability Limit Load Flows." Proceedings of USSR National Scientific and Technical Conference "Problems of Stability and Reliability of the USSR Power System", Dushanbe, 1989 - in Russian.

Kontorovich, A.M., Y.V. Makarov and A. A. Tarakanov. 1982. "Improvements of a Permanent Loading Technique to Compute Load Flows on Stability Margin." Trudy LPI, no. 380, pp. 37-41, 1982 - in Russian.

Kothari, D.P., and I.J. Nagarath. 2011. Modern Power System Analysis (4th Edition). McGraw Hill Press, Columbus, $\mathrm{OH}$.

Kravchuk, L., and J. Pierce. 2009. Transient Stability Analysis of a System with Wind Generation. MS Project, Dept. of Electrical and Electronic Engineering, University of California, Sacramento, CA.

Kroposki, B. 2009. "Renewable Energy Interconnection and Storage - Technical Aspects." NREL, Golden, CO. Presentation, Available http://www.sandia.gov/regis/presentations/B Kroposki-NREL.pdf. Accessed November 10, 2012.

Kroposki, B., T. Basso, and R. DeBlasio. 2008. "Microgrid Standards and Technologies." IEEE Power and Energy Society General Meeting - Conversion and Delivery of Electrical Energy in the 21st Century, Pittsburgh, PA, 20-24 July 2008, pp. 1-4. Institute of Electrical and Electronics Engineers, Piscataway, NJ.

Kumar, R., A. Ipahchi, V. Brandwajan, M. A. El-Sharkawi, and G. Cauley. 1991. "Neuron Networks for Dynamic Security Assessment of Large Scale Power Systems: Requirements Overview." In Proceedings of 1st International Forum on Applications of Neuron Networks to Power Systems, Seattle, WA, pp. 65-71, July 1991. Institute of Electrical and Electronics Engineers, Piscataway, NJ.

Kundur, P. 1994. Power System Stability and Control. McGraw-Hill, NY.

Kurita, A., and T. Sakurai. 1988. "The Power System Failure on July 23, 1987, in Tokyo." In Proceedings of the 27th Conference on Decision and Control, December 1988. Institute of Electrical and Electronics Engineers, Piscataway, NJ.

Kurth, R.D., Robert B. Henderson, R.S. Burton, Brent S. Hancock, and James N. Roik. 2005. Design, Implementation and Commissioning of a Remedial Action Scheme. DistribuTECH Conference and Exhibition, January 25-27, 2005. Available 
http://www.teshmont.com/pdf/papers/Distributech\%202005\%20Paper.pdf. Accessed November 28, 2012.

Labbate, A., and G. Fulli. 2010. "Modeling and application of VSC-HVDC in the European Transmission System." International Journal of Innovations in Energy Systems and Power, vol. 5, no. 1, pp. 8-16, April.

Labeau, P.E., C. Smidts, and S. Swaminathan. 2000. "Dynamic Reliability: Towards an Integrated Platform for Probabilistic Risk Assessment." Reliability Engineering \& System Safety, vol. 68, pp. 219-254, 2000.

Lalor, G., A. Mullane, and M. O'Maley. 2005. "Frequency Control and Wind Turbine Technology." IEEE Transactions on Power Systems, vol. 20, no. 4.

Lam, L.T., et al. 2007. "VRLA Ultrabattery for High-Rate Partial-State-Of-Charge Operation." Journal of Power Sources, 174: pp. 16-29.

Larruskain, D.M. et al., 2005. "Transmission and Distribution Networks: AC versus DC." In Proceedings 9th Spanish-Portuguese Congress on Electrical Engineering, Power Engineering Society, Marbella, Spain.

Larruskain, D.M., I. Zamora, O. Abarrategui, A. Iraolagoitia, M. D. Gutiérrez, E. Loroño, and F. de la Bodega. 2007. "Power Transmission Capacity Upgrade of Overhead Lines." Department of Electrical Engineering, E.U.I.T.I., University of the Basque Country, Available http://www.icrepq.com/icrepq06/296 Larruskain.pdf. Accessed November 20, 2012.

Larsson, S. and Danell, A. 2006. The Black-Out in Southern Sweden and Eastern Denmark. IEEE Power Systems Conference and Exposition," pp. 309-313.

Le, X., P.M.S. Carvalho, L.A.F.M. Ferreira, J. Liu, B.H. Krogh, N. Popli, and M.D. Ilić. 2010. "Wind Integration in Power Systems: Operational Challenges and Possible Solutions." Proceedings of the IEEE, vol. 99, no. 1, October 21. Institute of Electrical and Electronics Engineers, Piscataway, NJ.

Leclercq, L., C. Sudemont, B. Robyns, G. Cimuca, and M.M. Rabulescu. 2003. "Flywheel Energy Storage System to Improve the Integration of Wind Generators into a Network." In Proceedings of 5th International Symposium on Advanced Electromechanical Electromotion Systems ELECTROMOTION 2003, Marrakesh, Marocco, November 26-28, 2003.

Lee, S.T. 2003. "Community Activity Room as a New Tool for Transmission Operation and Planning Under a Competitive Power Market." In Proceedings of 2003 IEEE Bologna Power Tech Conf., vol. 4, June 23-26, 2003. Institute of Electrical and Electronics Engineers, Piscataway. NJ.

Leite da Silva, A.M., V.L. Arienti, and R.N. Allan. 1990. "Probabilistic Load Flow Techniques Applied to Power System Expansion Planning." IEEE Transactions on Power Systems, vol. 5, no. 4, pp. 1047-1053, November 1990.

Leite da Silva, A.M., V.L. Arienti, and R.N. Allan 1984. "Probabilistic Load Flow Considering Dependence Between Input Nodal Powers." IEEE Transactions on Apparatus and Systems, PAS-103, pp. 1524-1530, June 1984. 
Leite da Silva, A.M., L.A.F. Manso, Sales, W.S. Resende, L.C. Aguiar, M.J.Q Matos, M.A. Lopes, J.A. Peças and Miranda, V. 2004. "Application of Monte Carlo Simulation to Generating System Well-Being Analysis Considering Renewable Sources." International conference on probabilistic methods applied to power systems, pp. 439-444, September 2004.

Leite da Silva, A.M., A.C.G. Melo and S.H.F. Cunha. 1991. "Frequency and Duration Method for Reliability Evaluation of Large-Scale Hydrothermal Generating Systems." IEEE Proceedings C: Generation, Transmission and Distribution, vol.138, no.1, pp.94-102, January 1991. Institute of Electrical and Electronic Engineers, Piscataway, NJ.

Leite da Silva, A.M., L.C. Resende, L.A.F. Manso and R. Billinton. 2004. "Well-Being Analysis for Composite Generation and Transmission Systems." IEEE Transactions on Power Systems, vol. 19, no. 4, pp. 1763-1770, November 2004.

Lescale, V.F., A. Kumar, L.-E. Juhlin, H. Björklund, and K. Nyberg. 2008. "Challenges with MultiTerminal UHVDC Transmissions." POWERCON 2008 \& 2008 IEEE Power India Conference, New Delhi, India, October 12-15, 2008. Institute of Electrical and Electronics Engineers, Piscataway, NJ.

Lewis, H. and A.J. Salkind. 1997. "Cellulosic Separator Applications: New and Improved Separators for Alkaline Rechargeable Cells," J. Power Sources, 65, pp. 29-38.

Lex, P. 2005, Demonstration of a 2-MWh Peak Shaving Z-BESS.

Li, H., and Z. Chen. 2008. "Overview of Different Wind Generator Systems and Their Comparisons." IET Renewable Power Generation, vol. 2, no. 2, pp. 123-138.

Li, L. et al. 2011. "A Stable Vanadium Redox-Flow Battery with High Energy Density for LargeScale Energy Storage." Adv. Energy Mater., vol. 1, pp. 394-400.

Li, P., Y. Yu, H. Jia, J. Su, and J. Zhao. 2002. "A Study on Boundary of Small Disturbance Stability Region." In Proceedings International Conf. on Power Syst. Technology PowerCon 2002, vol. 2, October 13-17, 2002, pp. 1228 -1232. Institute of Electrical and Electronics Engineers, Piscataway, NJ.

Li, W. 2005. Risk Assessments of Power Systems - Models, Methods, and Applications. Ch 6, pp. 150, John Wiley \& Sons, Hoboken, New Jersey, 2005.

Li, W. and P. Choudhury. 2007. "Probabilistic Transmission Planning." IEEE Power and Energy Magazine, vol. 5, no. 5, pp. 46-53, September-October 2007.

Li, Y. and V. Venkatasubramanian. 2004. "Coordination of Transmission Path Transfers." IEEE Transactions on Power Systems, vol. 19, issue. 3, August 2004.

Lin, J., G. Damato, and P. Hand. 2011. "Energy Storage - a Cheaper, Faster, \& Cleaner Alternative to Conventional Frequency Regulation." CESA, editor. Strategen Consulting, Berkeley, CA.

Lin, Y. 2012. "Introduction to Generic Wind Turbine Generator Models." May, 2011, Siemens Energy, Inc. Available https://www.pti- 
us.com/pti/company/enewsletter/2011may/pdfs/Introduction\%20to\%20Generic\%20Wind\%20Mo dels.pdf. Accessed November 8, 2012

Lindahl, S. 2004. "Case studies of recent blackouts." CRIS International Workshop on Power System Blackouts - Causes, Analyses, and Countermeasures, Lund, Sweden, May 3, 2004.

Linden, D. 1995. Handbook of Batteries. ed. D. Linden. McGraw-Hill, New York.

Lindgren, G. and H. Rootzen. 1987. "Extreme values: Theory and technical applications." Scandinavian Journal of Statistics, Theory and Applications 14:241-279

Lings, R. 2005. "Overview of Transmission Lines Above 700 kV." Power Engineering Society Inaugural Conference and Exposition in Africa, vol., no., pp. 33-43, 11-15 July 2005. Institute of Electrical and Electronics Engineers, Piscataway, NJ.

Liu, H. and J. Jiang. 2007. "Flywheel Energy Storage - An Upswing Technology for Energy Sustainability," Energy and Buildings, pp. 599-601.

Löf, P.A., T. Smed, G. Andersson, and D.J. Hill. 1992. "Fast Calculation of a Voltage Stability Index." IEEE Trans. on Power Syst., vol. 7, no. 1, pp. 54-64, February 1992.

Long, J.W. 2008. "Electrochemical Capacitors Empowering the 21st Century." The Electrochemical Society Interface, Spring, pp. 33.

Lu, N, Makarov, Y.V, Weimar, M. R, Rudolph, F., Murthy, S, Arseneaux, J, Loutan, C, and Chowdhury, S. 2010, The Wide-Area Energy Storage and Management System Phase II, Final Report-Flywheel Field Tests. PNNL-19669, Pacific Northwest National Laboratory, Richland, WA. Available http://www.pnl.gov/main/publications/external/technical reports/PNNL-19669.pdf. Accessed December 2, 2012.

Lu, S., Y. V. Makarov, C. A. McKinstry, A. J. Brothers, and S. Jin. 2009. Low Probability Tail Event Analysis and Mitigation in BPA Control Area. PNNL-18769, Pacific Northwest National Laboratory, Richland, WA. Available http://www.pnl.gov/main/publications/external/technical reports/PNNL-18769.pdf. Accessed November 15, 2012.

Lu, W. and B. T. Ooi. 2001, "Multi-Terminal DC Transmission System For Wind-Farms." IEEE Power Engineering Society Winter Meeting, vol.3, Columbus, OH , January 28 - February 1, vol.1, pp.1091-1096.

Lucas, G. and H. Miller. 2010. "Dresser-Rand's SMARTCAES Compressed Air Energy Storage Solution." 2nd Compressed Air Energy Storage Conference \& Workshop, Columbia University, NY, October 20 and 21, 2010.

Macchi, E. and G. Lozza. 1987. "A Study of Thermodynamic Performance of CAES Plants, Including Unsteady Effects." Presented at the Gas Turbine Conference and Exhibition, Anaheim, California, May 31-June 4, 1987. American Society of Mechanical Engineers, NY.

Madaeni, S.H., R. Sioshansi, and P. Denholm. 2012. "Estimating the Capacity Value of Concentrating Solar Power Plants: A Case Study of the Southwestern United States." IEEE Transactions on Power Systems, vol. 27, no. 2, pp. 1116-1124. 
Madaeni, S.H., R. Sioshansi, and P. Denholm. 2012. "Estimating the Capacity Value of Concentrating Solar Power Plants With Thermal Energy Storage: A Case Study of the Southwestern United States." IEEE Transactions on Power Systems, vol. PP, no. 99, 2012.

Madrigal, M., K. Ponnambalam, and V.H. Quintana. 1998. "Probabilistic optimal Power Flow." In Proceedings of IEEE Canadian Conference on Electrical and Computer Engineering, vol. 1, pp. 385-388, May. Institute of Electrical and Electronics Engineers, Piscataway, NJ.

Maher, M. and V. VanZandt. 2009. "Opportunity - Smart Grid Investment Grant. The Western Interconnection Synchrophasor Program (WISP): Western Interconnect Synchrophasor Program NASPI Roadmap." Status Briefing for the WECC Board of Directors, October 22, 2009. Available http://www.wecc.biz/committees/BOD/102109/Lists/Presentations/1/09\%20SynchroPhasor\%20 Presentation.pdf. Accessed November 15, 2012.

Makarov, Y.V., P.V. Etingov, N. Zhou, J. Ma, N.A. Samaan, R. Diao, S. Malhara, R.T. Guttromson, P. Du, and C. Sastry. 2010a. Analysis Methodology for Balancing Authority Cooperation in High Penetration of Variable Generation. PNNL-19229, Pacific Northwest National Laboratory, Richland, WA. Available http://www.pnl.gov/main/publications/external/technical reports/PNNL-19229.pdf.

Makarov, Y..V., S. Lu, X. Guo, J.F. Gronquist, P. Du, T.B. Nguyen, and J.W. Burns. 2010 b. Wide Area Security Region Final Report. PNNL-19331, Pacific Northwest National Laboratory, Richland, WA. March. Available http://www.pnl.gov/main/publications/external/technical reports/PNNL-19331.pdf.

Makarov, Y.V., J. Ma, S. Lu, and T. B. Nguyen, 2008a. Assessing the Value of Regulation Resources Based on Their Time Response Characteristics. PNNL-17632, Pacific Northwest National Laboratory, Richland, WA.

Makarov, Y.V., J.H. Pease, C. Loutan, T. VanBlaricom, and C. Potter. 2008b. Notes on Visit to Red Eléctrica de España (REE). PNNL-17680, Pacific Northwest National Laboratory, Richland, WA, July 2008.

Makarov, Y.V., B. Yang, J. DeSteese, S. Lu, C. Miller, P. Nyeng, J. Ma, D. Hammerstrom, V. Viswanathan. 2008c. Wide-Area Energy Storage and Management System to Balance Intermittent Resources in the Bonneville Power Administration and California ISO Control Areas. PNNL-17574, Pacific Northwest National Laboratory, Richland, WA. June 2008. Available http://www.pnl.gov/main/publications/external/technical reports/PNNL-17574.pdf. Accessed December 3, 2012.

Makarov, Y.V., D.J. Hill, and Z.Y. Dong. 2000a. "Computation of Bifurcation Boundaries for Power Systems: A New $\Delta$-Plane Method." IEEE Trans. on Circ. and Syst. I: Fundamental Theory and Applications, vol. 47, issue 4, April 2000, pp. 536-544.

Makarov, Y.V., D.J. Hill, and I.A. Hiskens. 2000b. "Properties of Quadratic Equations and Their Application to Power System Analysis." Electrical Power and Energy Syst., 22 (2000), pp. 313323. 
Makarov, Y.V., Q. Wu, D.J. Hill, D.H. Popovic and Z.Y. Dong. 1997. "Coordinated Steady-State Voltage Stability Assessment and Control." In Proceedings of International Conference on Advances in Power System Control, Operation and Management APSCOM'97, Wanchai, Hong Kong, November 11-14, 1997. Institute of Electrical and Electronics Engineers, Piscataway, NJ.

Makarov, Y.V., V.A. Maslennikov, and D.J. Hill. 1995. "Calculation of Oscillatory Stability Margins in the Space of Power System Controlled Parameters." In Proceedings of the International Symposium on Electric Power Engineering Stockholm Power Tech, vol. Power Systems, Stockholm, Sweden, pp. 416-422, June 1995. Institute of Electrical and Electronics Engineers, Piscataway, NJ.

Makarov, Y.V., and I. A. Hiskens. 1994. "A Continuation Method Approach to Finding the Closest Saddle Node Bifurcation Point." In Proceedings of NSF/ECC Workshop on Bulk Power System Voltage Phenomena III, Davos, Switzerland, August 1994. ECC Inc., Fairfax, Virginia.

Mansour, Y., A.Y. Chang, J. Tamby, E. Vaahedi, B.R. Corns, and M.A. El-Sharkawi. 1997. "Large Scale Dynamic Security Screening and Ranking Using Neuron Networks." IEEE Transactions on Power Systems, vol. 12, no. 2, pp. 954-960, May.

Maria, G.A., C. Tang, and J. Kim. 1990. "Hybrid Transient Stability Analysis [Power Systems]." IEEE Transactions on Power Systems, vol. 5, no. 2, pp. 384-393.

Martin, L. 2010. "Grid Integration of Large Scale Wind and Solar." Siemens presentation, Asia Pacific Clean Energy Summit and Expo, Honolulu, Hawaii, August 30-September 2, 2010. Available http://www.ct-si.org/events/APCE/ State of Hawaii, Honolulu, Hawaii.

Masiello, R. 2004. "Utilities Must Leverage Existing Resources and Upgrade Technology to Avoid Future Blackouts." Electric Energy T\&D Magazine, pp. 44-47. May/June. Matveev, V.A. 1964. "A Method of Numeric Solution of Sets of Nonlinear Equations." Zhurnal Vychislitelnoi Matematiki I Matematicheskoi Fiziki, vol. 4, no. 6, pp. 983-994, 1964 - in Russian.Van Cutsem, T. and C. Vournas. 1998. "Voltage Stability of Electric Power Systems." Kluwer Academic Publishers (Power Electronics and Power Systems Series), Boston, Mass.

Maxwell, M., and M. Cohen. 2011. "PEM Fuel Cell Systems Providing Backup Power to Commercial Cellular Towers and an Electric Utility Communications Network." In DOE Hydrogen and Fuel Cells Program Annual Progress Report, DOE, Washington, D.C. Editor. Intelligent Energy, Long Beach CA. pp. 1304-1307.

Mayadas-Dering, J. 2009. "NY Utility Examines Integrating Dynamic Line Ratings." Utility Automation \& Engineering T\&D Magazine, July Vol 14, issue 7.

McCall, J. 2009. "DC Superconductor Cables for Long Distance Transmission." American Superconductor presentation, Fall EEI Transmission, Distribution, \& Meeting Conference, Kansas City, KS. http://www.eei.org/meetings/Meeting\%20Documents/2009FallTDM-3McCall,\%20Jack.pdf. Accessed November 20, 2012.

McCall, J. Gamble, B. and Eckroad, S. 2010 "Combining Superconductor Cables and VSC HVDC Terminals for Long-Distance Transmission," IEEE Conference on Innovative Technologies for an Efficient and Reliable Electricity Supply (CITRES), September 2010, pp. 47-54. 
McCalley, J.D., S. Wang, Q. L. Zhao, G. Z. Zhou, R.T. Treinen, and A. D. Papalexopoulos. 1997. "Security Boundary Visualization for Systems Operation." IEEE Transactions on Power Systems, vol. 12, no. 2, May 1997. Institute of Electrical and Electronics Engineers, Piscataway, NJ, pp. 940-947.

McCalley, D., and V. Vittal. 1998. Risk-based Security Assessment. EPRI Final Report W0860401, Electric Power Research Institute, Palo Alto, CA, December 1998.

McDonough, R.N., and Anthony D. Whalen.1995. "Detection of Signals in Noise." Academic Press, San Diego, California.

Measure, B.A., R. Whiting, W. B. Booth, James A. Yost, T. Karier, P. Rockefeller, B. Bradbury, and J. M. Dukes. 2011. "Wind Integration Forum Steering Committee Meeting Notes, June 6, 2011." Available http://www.nwcouncil.org/energy/wind/meetings/2011/06/WIF\%20SC\%20611\%20Notes\%20Final.pdf. Accessed November 8, 2012.

Meegahapola, L, T. Littler, B. Fox, J. Kennedy, and D. Flynn. 2010. "Voltage and Power Quality Improvement Strategy for a DFIG Wind Farm during Variable Wind Conditions." In Proceedings of the International Symposium Modern Electric Power Systems (MEPS), Wroclaw, Poland, September 20-22, 2010. Institute of Electrical and Electronics Engineers, Piscataway, NJ.

Meliopoulos, A.P. Sakis, G. J. Cokkinides, and X.Y. Chao. 1990. "A New Probabilistic Power Flow Analysis Method." IEEE Transactions on Power Systems, vol. 5, no. 1, pp. 182-190, February.

Mensah-Bonsu, C. 2000. "Instrumentation and Measurement of Overhead Conductor Sag using the Differential Global Positioning Satellite System." Doctoral Dissertation, Dept. Philosophy, Arizona State University, Tempe, AZ.

Midwest ISO. 2011. "Proposed Multi Value Project Portfolio." Midwest ISO Presentation, Business Case Workshop, September 29, 2011. MISO, Carmel, IN. Available https://www.midwestiso.org/Library/Repository/Meeting\%20Material/Stakeholder/MVP/2011\%20 MVP/20110919/20110919\%20MVP\%20Proposed\%20Portfolio\%20Business\%20Case.pdf.

Accessed November 20, 2012.

Miller, N., K. Clark, and M. Shao. 2010. "Impact of Frequency Responsive Wind Plant Controls on Grid Performance. Transmission Planning for Wind Energy: Status and Prospects." Proceedings of the $9^{\text {th }}$ International Workshop on Large Scale Integration of Wind Power and Transmission Networks for Offshore Wind Farms, pp. 348-355, October 2010. Hydro Quebec, Quebec, Canada.

Milligan, M.R. 1996. Measuring Wind Plant Capacity Value. Tech. Rep. NREL/TP-441-20493, National Renewable Energy Laboratory, Golden, CO.

Milligan, M.R., and T. Factor. 2000. "Optimizing the Geographic Distribution of Wind Plants in Iowa for Maximum Economic Benefit and Reliability." Wind Engineering, vol. 24, pp. 271-290, July.

Milligan, M.R., and B. Parsons. 1999. "Comparison and Case Study of Capacity Credit Algorithms for Wind Power Plants." Wind Engineering, vol. 23, pp. 159-166, May. 
Milligan, M.R. and K. Porter. 2006. "The Capacity Value of Wind in the United States: Methods and Implementation." The Electricity Journal, vol. 19, pp. 91-99, March.

Mitlitsky, F. et al. 1998. "Unitized Regenerative Fuel Cell Systems." In 1998 Fuel Cell Seminar. 1998. Palm Springs, CA.

Mizumori, M. and B. Nickell. 2008. "Balancing Authority Proliferation." RPIC Meeting, WECC, November 13, 2008. Available http://www.wecc.biz/committees/BOD/RPIC/111308/Lists/Agendas/1/7 BA\%20Proliferation.pdf. Accessed November 15, 2012.

Mokhoff, N. 2011. "Smart Grid: Slow Buildout Begins." Electronic Engineering Times, pp. 22-28.

Molinski, T. 1996. "Geomagnetically Induced Currents: Cause, Effect, and Mitigation." IEEE Canadian Review, no. 25, pp. 11-14, Fall.

Morales, J.M., and J. P'erez-Ruiz. 2005. "Point Estimate Schemes to Solve the Probabilistic Power Flow." IEEE Transactions on Power Systems, vol. 22, no. 4, November.

Morgan, M., I. Dobson, R. Adapa, B. Carreras, V. Dawar, M. Kumbale, R. Hardiman, L. Hwang, B. Lesieutre, J. Kim, Y. Makarov, N. Samaan, D. Newman, and S.Varadan. 2011. Extreme Events Phase 2. Prepared for the California Energy Commission by Pacific Northwest National Laboratory, University of Wisconsin-Madison, Electric Power Research Institute, BACV Solutions, Southern Company, CIEE/PIER, University of Alaska - Fairbanks, and KEMA. CECMR-08-03. Available http://uc-ciee.org/downloads/Extreme Events Phase 21.pdf Accessed November 26, 2012.

Morton, A.B. 2009. "Wind Turbines and Oscillatory Stability in Power Systems: Towards a General Approach." In Proceedings of the 2009 Australasian Universities Power Engineering Conference (AUPEC 2009), pp. 6, 2009. Institute of Electrical and Electronics Engineers, Adelaide, SA.

Muljadi, E., C.P. Butterfield, B. Parsons, et al. 2007. "Effect of Variable Speed Wind Turbine Generator on Stability of a Weak Grid." IEEE Transactions on Energy Conversion, vol. 22, no. 1, pp. 29-36.

Murty, M.S.R. undated. "Wind Turbine Generator Model." Available http://www.sarienergy.org/PageFiles/What We Do/activities/CEB Power Systems Simulation Training, Colo mbo, Sri Lanka/Course ppts/Wind TG Model.pdf. Accessed November 27, 2012.

Myska, D. 2009. "Allocation Algorithm Review." In Proceedings of 2nd Workshop for Market Participants, Central Allocation Office GmbH, Munich, Germany, August 12, 2009. Available http://centralao.com/index.php?option=com content \&view=category\&layout=blog\&id=51\&ltemid=114. Accessed November 15, 2012.

Nakhamkin, M. "Second Generation of the CAES Technology." Compressed Air Energy Storage (CAES) Conference \& Workshop, Columbia University, October 21-22, 2008. Columbia University, NY. 
Nakhamkin, M., M. Chiruvolu, and C. Daniel. 2007. "Available Compressed Air Energy Storage (CAES) Plant Concepts." Available www.espcinc.com/library/PowerGen 2007 paper.pdf. Accessed November 20, 2012.

Nakhamkin, M., M. Chiruvolu, M. Patel, S. Byrd, and R. Schainker. 2009. "Second Generation of CAES Technology: Performance, Operations, Economics, Renewable Load Management, Green Energy." POWER-GEN International, December 8-10, 2009. Energy Storage Power Corporation, Las Vegas Convention Center, Las Vegas, NV.

Nakhamkin, M., B. Kraft, and C. Moran. 2010. "Advanced Second Generation of CAES Technology 180 MW, 310 MW and 450 MW CAES Plants." 2nd Compressed Air Energy Storage (CAES) Conference \& Workshop, Columbia University, October 20-21, 2010, New York State Energy Research and Development Authority, New York City, NY.

Naoi, K. 2010. "NAnohybri Capacitor: The Next Generation Electrochemical Capacitors." Fuel Cells, 10(5): pp. 825-833.

Naoi, K. 2007. "Available Compressed Air Energy Storage (CAES) Plant Concepts." Presented at PowerGen 2007. Available www.espcinc.com/library/PowerGen 2007 paper.pdf.

Naoi, K., and N. Anohybri. 2010. "Capacitor: The Next Generation Electrochemical Capacitors." Fuel Cells, 10(5):p. 825-833.

Naoi, K. and M. Morita. 2008. "Advanced Polymers as Active Materials and Electrolytes for Electrochemical Capacitors and Hybrid Capacitor Systems." The Electrochemical Society Interface, Spring: pp. 44-48.

Naoi, K., and P. Simon. 2008. "New Materials and New Configurations for Advanced Electrochemical Capacitors." The Electrochemical Society Interface, Spring: pp. 34-37.

NERC. 2012. "Reliability Indicators: Planning Reserve Margin." NERC, Princeton, NJ. Available http://www.nerc.com/page.php?cid=4\%7C331\%7C373. Accessed November 20, 2012.

NERC. 2011. Demand Response Availability Data System (DADS): Phase I \& II Final Report. January. Available. Accessed November 28, 2012.

NERC. 2010a. Potential Reliability Impacts of Emerging Flexibility Resources. Special Report, NERC, Princeton, NJ, November 2010. Available http://www.nerc.com/docs/pc/ivgtf/IVGTF Task 15 Final.pdf. Accessed November 20, 2012.

NERC. 2010b. Flexibility Requirements and Metrics for Variable Generation: Implications for System Planning Studies. Draft Report, Task Force 1-4, NERC Integration of Variable Generation Task Force (IVGTF), NERC, Princeton, NJ. June 2010.

NERC. 2010c. Generator Verification, Available http://www.nerc.com/filez/standards/GeneratorVerification-Project-2007-09.html. Accessed November 15, 2012.

NERC. 2010d. "Integrated Bulk Power System Risk Assessment Concepts." White Paper. NERC, Princeton, NJ. Available http://www.nerc.com/docs/pc/rmwg/lntegrated Bulk Power System Risk Assessment Conce pts Final.pdf. Accessed November 26, 2012. 
NERC. 2009a. Accommodating High Levels of Variable Generation. NERC Integration of Variable Generation Task Force (IVGTF), NERC, Princeton, NJ. April 2009. Available http://www.nerc.com/files/IVGTF Report 041609.pdf. Accessed November 1, 2012.

NERC. 2009b. A Technical Reference Paper Fault- Induced Delayed Voltage Recovery. Version 1.2, Transmission Issues Subcommittee and System Protection and Control Subcommittee, NERC, Princeton, NJ June 2009. Available http://www.nerc.com/docs/pc/tis/FIDVR Tech Ref\%20V1-2 PC Approved.pdf. Accessed November 1, 2012.

NERC. 2009c. "Standard PRC-024-1 - Generator Frequency and Voltage Protective Relay Settings." NERC, Princeton, NJ. Available http://www.nerc.com/docs/standards/sar/PRC-0241 Draft1 2009Feb17.pdf. Accessed November 12, 2012.

NERC. 2009d. Demand Response Availability Data System (DADS) Phase I \& II. North American Electric Reliability Corporation, September 1, 2009.

NERC. 2007. "Definition of Adequate Level of Reliability." NERC, Princeton, NJ, December 2007. Available http://www.nerc.com/docs/pc/Definition-of-ALR-approved-at-Dec-07-OC-PCmtgs.pdf. Accessed November 26, 2012.

NERC. 2004a. Technical Analysis of the August 14, 2003, Blackout: What Happened, Why, and What Did We Learn?, Report to the NERC Board of Trustees by the NERC Steering Group, July 13, 2004.

NERC. 2004b. Final Report on the August 14, 2003 Blackout in the United States and Canada: Causes and Recommendations. U.S. - Canada Power System Outage Task Force, April 5, 2004. Available http://www.nerc.com/docs/docs/blackout/Blackout-OneYearLater.pdf. Accessed December 6, 2012.

NERC. 2004c. Resource and Transmission Adequacy Recommendations. Prepared by the Resource and Transmission Adequacy Task Force, NERC Planning Committee, Village Boulevard, NJ, NERC, June 15, 2004. Available

http://www.nerc.com/docs/docs/pubs/Resource and Transmission Adequacy Recommendatio ns.pdf Accessed November 27, 2012.

NERC. 2004d. Frequency Response Standard Whitepaper. North American Electric Reliability Council, Princeton, NJ. April 2004. Available www.nerc.com/docs/oc/rs/Frequency Response White Paper.pdf. Accessed November 20, 2012.

NERC 1996a. Western Systems Coordinating Council Disturbance Report for the Power System Outage that Occurred on the Western Interconnection on August 10, 1996. October 18, 1996. NERC, Washington, D.C. Available http://www.nerc.com/docs/docs/pubs/AUG10FIN.pdf

NERC. 1996b. Glossary of Terms. Glossary of Terms Task Force, North American Electric Reliability Council, Princeton, NJ, August 1996. 
NERC. 2008. Reliability Standards, "WECC Standard BAL-002-WECC-1 - Contingency Reserves." Available http://www.nerc.com/files/BAL-002-WECC-1.pdf. Accessed November 28, 2012.

Neptune Regional Transmission System. Undated. Neptune Regional Transmission System. Available http://neptunerts.com/. Accessed November 15, 2012.

Ning, C.N., C. A. Hsieh, T. Y. Hsiao, and C. N. Lu. 2006. "Two Application Examples of Probabilistic Risk Assessment in Power System Operations." In Proceedings of International Conference on Probabilistic Methods Applied to Power Systems, Stockholm, Sweden, pp. 1-5.

Norris, B.J., R.J. Perry, and R.M. Hudson. 2004. "An Evaluation of Wind Farm Stabilization and Load Shifting Using the Zinc-Bromine Battery (ZBB)." Gridwise Engineering Company, Danville, CA.

Norris, B.L., et al. 2002. Grid-Connected Solar Energy Storage Using the Zinc-Bromine Flow Battery. Gridwise Engineering Company, Danville, CA.

Northwest Wind Integration Forum. 2007. Available http://www.nwcouncil.org/energy/Wind/Default.asp. Accessed October 30, 2012.

Oshima, T., and M. Kajita. 2004. "Development of Sodium-Sulfur Batteries." International Journal of Applied Ceramic Technology, vol. 1, no. 3, pp. 269-276.

"Pacific Northwest National Laboratory." PNNL Website. Available http://www.pnnl.gov Accessed November 26, 2012.

PacifiCorp Website, http://www.pacificorp.com/index.html. Accessed November 28, 2012.

Parashar, M. A. Agarwal, A. Makarov and, Y. V. Dobson, I. 2007. Real-Time Voltage Security Assessment (RTVSA): Summary Report. Prepared for California Independent System Operator Corporation, Folsom, CA, by Consortium for Electric Reliability Technology Solutions, January 21, 2008. Available http://certs.lbl.gov/pdf/task3-rtvsa-summary-rpt.pdf. Accessed November 15, 2012.

Parashar, M., A. Agarwal, Y. Makarov, and I. Dobson, Madison,. 2007a. Real-Time Voltage Security Assessment (RTVSA) Algorithms \& Framework. Prepared for California Independent System Operator, Folsom, CA, by Consortium for Electric Reliability Technology Solutions, December 2007. Available http://certs.lbl.gov/certs-rt-pubs.html. Accessed November 1, 2012.

Parashar, M., A. Agarwal, Y. Makarov, and I. Dobson. 2007b. Real-Time Voltage Security Assessment Tool (RTVSA) Functional Specifications For Commercial Grade Application. Prepared for California Independent System Operator, Folsom, CA ,by Consortium for Electric Reliability Technology Solutions, April 9, 2007. Available http://certs.lbl.gov/certs-rt-pubs.html Accessed 11/27/2012.

Parker, C.J., I.F. Morrison, and D. Sutanto. 1996. "Application of an Optimization Method for Determining the Reactive Margin from Voltage Collapse in Reactive Power Planning." IEEE Transactions on Power Systems, vol. 11, no. 3, pp. 1473-1481, August 1996. 
Pavlov, A., L. Grigorieva et al., 1996. "Ni-Zn Batteries with Long Cycle Life." Journal of Power Sources, 62:pp. 113-116.

POWERnews. 2009. "China to Triple Ultra-High-Voltage Transmission Lines by 2012." Power News, March 4, 2009. Available http://www.powermag.com/POWERnews/China-to-Triple-UltraHigh-Voltage-Transmission-Lines-by-2012 1773.html. Accessed November 6, 2012.

Powertech Labs. 2008. VSAT - Voltage Security Assessment Tool. 2008 Available http://www.dsapowertools.com/downloads/VSAT Brochure.pdf. Accessed November 27, 2012.

PowerWorld Corporation. Undated. PowerWorld Simulator - PVQV. Available http://www.powerworld.com/products/simulator/add-ons-2/simulator-pvqv. Accessed November 27, 2012.

Prodromidis, A. and F.A. Coutelieris. 2011. "Simulations of Economical and Technical Feasibility of Battery and Flywheel Hybrid Energy Storage Systems in Autonomous Projects." Renewable Energy, pp. 149-153, 2011.

Propst, J.E. 1995. "Calculating Electrical Risk And Reliability." IEEE Transactions on Industry Applications, vol. 31, pp. 1197-1205, 1995.

Pudaruth, G.R., and F. Li. 2008. "Capacity Credit Evaluation: A Literature Review" In Third International Conference on Electric Utility Deregulation and Restructuring and Power Technologies. Nanjing, China, pp. 2719-2724, April 6-9, 2008. Institute of Electrical and Electronics Engineers, Piscataway, NJ.

Rabih, S., O. Ralliers, C. Turpin, S. Astier. 2008. "Experimental Study of a PEM Reversible Fuel Cell." In Proceedings of the International Conference on Renewable Energy and Power Quality (ICREPQ'08, Valencia; March 11-12, 2008, pp. 268.

Rahmatullah, N. 2006. Grid Reinforcing Wind Generation. Thesis for the Degree of Licentiate of Engineering, Department of Energy and Environment, Chalmers University of Technology, Göteborg, Sweden.

Ramtharan, G., N. Jenkins, and O. Anaya-Lara et al. 2007. "Influence of Rotor Structural Dynamics Representations on the Electrical Transient Performance of FSIG and DFIG Wind Turbines." Wind Energy, vol. 10, no. 4, pp. 293-301, July-August.

Ranjbari, A. et al. 2010. "Recent Advances in the Development of Unitized Regenerative Fuel cells based on PEM technology." In 18th World Hydrogen Energy Conference. Essen, Germany. May 16-21, 2010.

Rastler, D. 2010. Electricity Energy Storage Technology Options: A White Paper Primer on Applications, Costs and Benefits. Rept \#1020676. Electric Power Research Institute, Palo Alto, CA.

Reactive Reserve Working Group. 2006. Guide to WECC/NERC Planning Standard I.D: Voltage Support and Reactive Power. Prepared by Reactive Reserve Working Group, WECC,

Washington, D.C. March 30, 2006. 
Rei, A.M., M. T. Schilling, and A. C. G. Melo,. 2006. "Monte Carlo Simulation and Contingency Enumeration in Bulk Power Systems Reliability Assessment." In Proceedings of the 9th International Conference on Probabilistic Methods Applied to Power Systems, Stockholm, Sweden, June 11-15, 2006.

Ridge Energy Storage \& Grid Services L.P. 2005. "The Economic Impact of CAES on Wind in TX, OK and NM." Final Report June 27, 2005. Report for Texas State Energy Conservation Office, Austin, TX.

Rohr, D. 2011a. "Accelerating Acceptance of Fuel Cell Backup Power Systems." In DOE Hydrogen and Fuel Cells Program Annual Progress Report, DOE, Washington, D.C. Editor. Plug Power, Inc., Latham, NY, pp. 1328-1329.

Rohr, D. 2011b. "Highly Efficient, 5 kW CHP Fuel Cells Demonstrating Durability and Economic Value in Residential and Light Commercial Applications." in DOE Hydrogen and Fuel Cells Program Annual Progress Report, DOE, Washington, D.C. Editor. Plug Power, Inc. Latham, NY, pp. 1325-1327.

Rosehart, W., C. Roman, and A. Schellenberg. 2005. "Optimal Power Flow with Complementarity Constraints." IEEE Trans. on Power Syst., vol. 20, no. 2 , pp. 813-822.

Rounds, R. and G.H. Peek. 2008. "Design \& Development of a 20-MW Flywheel-based Frequency Regulation Power Plant." Sandia Report SAND 2008-8229, Sandia National Laboratory, Albuquerque, NM.

Rudervall, R., J.P. Charpentier, and R. Sharma. 2012. "High-Voltage Direct Current (HVDC) Transmission Systems Technology Review Paper." Presented at Energy Week 2000, Washington, DC, 7-8 March 2000. Available http://www2.internetcad.com/pub/energy/technology abb.pdf. Accessed November 10, 2012.

Rueda, J.L., and F. Shewarega. 2009. "Small Signal Stability of Power Systems with Large Wind Power Integration." CIGRE, .Paris, France.

Ruiz-Vega, R., A.R. Messina, and M. Pavella. 2004. "On-Line Assessment and Control of Transient Oscillations Damping." IEEE Transactions on Power Systems, vol. 19, issue. 2, May 2004, pp. 1038-1047.

Rydh, C.J. 1999. "Environmental Assessment of Vanadium Redox and Lead-Acid Batteries for Stationary Energy Storage." Journal of Power Sources, vol. 80, pp. 21-29.

Salamaa, M.M., E.M. Saieda, M.M. Abou-Elsaada, and E.F. Gharianyb. 2001. "Estimating the Voltage Collapse Proximity Indicator Using Artificial Neural Network." Energy Conversion and Management, vol. 42, no. 1, pp. 69-79, January.

Salasovich, J., and Mosey. G., 2011. "Feasibility Study of Solar Photovoltaics on Landfills in Puerto Rico." NREL, August 2011. Available http://www.nrel.gov/docs/fy11osti/52181.pdf. Accessed December 5, 2012.

Sanabria, L.A., and T.S. Dillon. 1993. "Power System Reliability Assessment Suitable for a Deregulated System Via the Method Of Cumulants." Electric Power and Energy Systems, vol. 20, pp. 203-211, February. 
Sayas, F.C., and R.N. Allan. 1996. "Generation Availability Assessment of Wind Farms." IEEE Proceedings C: Generation, Transmission and Distribution, vol. 143, no. 5, pp. 507-518, September 1996. Institute of Electrical and Electronics Engineers, Piscataway, NJ.

Sauer, P,.W. and B. C. Lesieutre. 1995. "Power System Load Modeling." in J. H. Chow, P. V. Kokotovic and R. J. Thomas, (Editors), Systems and Control Theory for Power Systems, Springer-Verlag, pp. 283-313.

Sauer, P.W., K. Tomsovic, J. Dagle, S. Widergren, T. Nguyen, and L. Schienbein, 2004. Integrated Security Analysis. Final Report, CERTS, July 2004. Available http://certs.lbl.gov/CERTS P RealTime.html. Accessed November 27, 2012.

Schainker, R.B. W.J. Steeley, and V, M-Wray, 2010. "Insights from EPRI's CAES Economic Benefit-Cost Analyses." 2nd Compressed Air Energy Storage (CAES) Conference \& Workshop, Columbia University, NY. October 20-21, 2010.

Schellenberg, A., W. Rosehart, and J. Aguado. 2004. "Cumulant Based Probabilistic Optimal Power Flow (P-OPF)." In Proceedings of $8^{\text {th }}$ International Conference on Probabilistic Methods Applied to Power Systems, September 2004. Iowa State University, Ames, Iowa.

Scaini, V, P. Lex, T. Rhea and N. Clark. 2002. "Battery Energy Storage for Grid Support Applications." In Proceedings of the Electrical Energy Storage Applications and Technologies (EESAT) 2002, San Francisco, CA, April 15- 17, 2002. Sandia National Laboratories, Albuquerque, NM.

Schilling, M.Th., A.M. Leite da Silva, R. Billinton, and M.A. El-Kafy. 1990. "Bibliography on power system probabilistic analysis (1962-1988)." IEEE Transactions on Power Systems, 5(1):1-11, February.

Schoenung, S.M. and W.V. Hassenzahl. 2003. "Long vs. Short-Term Energy Storage Technology Analysis." SAND2003-2783. Sandia National Laboratories, Albuquerque, NM.

Seale, R.V. 2007. "An Investigation into the Technical Design, Transient Stability Studies and Modeling Issues for Land Based WF Small Island Grid." The Barbados Light \& Power Company, Available http://www.google.com/url?sa=t\&rct=i\&q=r.v.\%20seale $\% 2 C \% 20 \% E 2 \% 80 \% 9$ Can $\% 20$ investigati on\%20into\%20the\%20technical\%20design\&source=web\&cd=1\&ved=0CC0QFiAA\&url=http\%3A \%2F\%2Fwww.caricom.org\%2Fisp\%2Fprojects\%2Fcredp\%2FRohan\%2520Seale\%2520Transie nt\%2520Stability\%2520Studies\%2520presentation.ppt\&ei=UhubULf8F-

S6iwK07oHQAw\&usg=AFQjCNEtT q4Qq f-CzqD1zoEpE x-ILhg. Accessed November 27, 2012.

Seppa, O. 2007. "Reliability and Real-Time Transmission Line Ratings." The Valley Group, NY. June 18, 2007.

Series Capacitor Working Group of the Transmission and Distribution Committee. 2010. Draft Guide for the Specification of Fixed Series Capacitor Banks for Transmission System Applications, IEEE P1726, January 2010. Available http://grouper.ieee.org/groups/td/cap/seriescap/IEEEP1726D10_20100129_FSCSpecGuide.do Accessed November 27, 2012. 
Shirmohamadi, M. 2002. "Sagging Line Mitigator Final Report." Consultant Report, California Energy Commission, Folsom CA, December 2002.

Sibley. L. undated. Patented Flywheel Energy Storage System - FESS. Tribology Systems Incorporated, Warminster, PA. Available http://www.tribologysystems.com/index files/TSI FESS Overview.pdf Accessed on 11/2712.

Simon, P. and A. Burke 2008. "Nanostructured Carbons: Double-Layer Capacitance and More." The Electrochemical Society Interface, Spring: pp. 38-42.

Simon, P. and Y. Gogotsi. 2008. "Materials for Electrochemical Capacitors." Nature Materials, 7:p. 845-854.

Singh, C., and A.L. Gonzalez. 1985. "Reliability Modeling of Generation Systems Including Unconventional Energy Sources." IEEE Transactions on Power Apparatus and Systems, vol. PAS-104, no. 5, pp. 1049-1056, May 1985.

Singh, B. and S.N. Singh. 2009. "Wind Power Interconnection into the Power System: A Review of Grid Code Requirements." The Electricity Journal, vol.22, no. 5, pp. 54-63, June.

Sittithumwat, A. and K. Tomsovic. 2002. "Dynamic Security Margin Estimation Using Artificial Neural Networks." IEEE Power Engineering Society Summer Meeting, vol. 3, pp. 1322-1327, 2002. Institute of Electrical and Electronics Engineers, Piscataway, NJ.

Skilling, J. 1998. Probabilistic Data Analysis: An Introductory Guide. Unpublished manuscript, Department of Applied Mathematics and Theoretical Physics, University of Cambridge , Cambridge, England. Available http://www.maxent.co.uk/documents/pda.pdf. Accessed October 30,2012

Skyllas-Kazacos, undated. The Vanadium Redox Flow Battery: Science and Materials. Available http://www.ccrhq.org/userfiles/file/Niche/Skyllas-Kazacosweb.ppt.pdf. Accessed October 31, 2001.

Slootweg, J.G. 2003. Wind Power, Modeling and Impact on Power System Dynamics. PhD Thesis, Power Systems Laboratory, Delft University of Technology, Ridderkerk, Netherlands.

Slootweg, J.G., and W. L. Kling. 2003. "The Impact of Large Scale Wind Power Generation on Power System Oscillations." Electric Power Systems Research, vol. 67, no. 1, pp. 9-20, October.

Smith, J.C. 2004. "Utilities and Wind Power Integration." Utility Wind Interest Group presentation at Stanford University, April 26, 2004. Available

http://gcep.stanford.edu/pdfs/energy workshops 04 04/wind c smith.pdf Accessed November 27, 2012.

Song, Y., H. He, D. Zhang, and J. Guo. 2006. "Probabilistic Security Evaluation of Bulk Power System Considering Cascading Outages." In Proceedings of International Conference on Power System Technology, pp. 1-6, 2006. Institute of Electrical and Electronics Engineers, Piscataway, NJ. 
Southwest Transmission Cooperative, Inc, 2005. "Standard Large Generator Interconnection Agreement (LGIA)." Benson, AZ. Available http://www.oatioasis.com/SWTC/SWTCdocs/LGIA.pdf. Accessed November 28, 2012.

Sovalov, S.A. and V. A. Semenov. 1988. Emergency Control in Power Systems. Energoatomizdat, Moscow, 1988 - in Russian.

Staudt, L. undated."Electricity Storage: Windfarm and Industrial Applications." Centre for Renewable Energy, Dundalk Institute of Technology. Co. Louth, Ireland. Available http://docs.wind-watch.org/El_Storage_Presentation.pdf. Accessed October 26, 2011.

Steeley, W. 2005. "VRB Energy Storage for Voltage Stabilization." Report 1008434, Electric Power Research Institute, Palo Alto, CA. March.

Stelmak, R. 2009. "Dynamic Line Ratings A Complementary Technology for SynchroPhasors." The Valley Group, NASPI Meeting Chattanooga, TN, pp. 3, October 7-8 2009.

Su, C-L. 2005. "Probabilistic Load-Flow Computation Using Point Estimate Method." IEEE Transactions on Power Systems, vol. 20, no. 4, November.

Su, J., Y. Yu, H. Jia, P. Li, N. He, Z. Tang, and H. Fu. 2002. "Visualization of Voltage Stability Region of Bulk Power System." In Proceedings of International Conference on Power System Technology PowerCon 2002, vol. 3, pp. 1665-1668. Institute of Electrical and Electronics Engineers, Piscataway, NJ.

Succar, S. and R. H. Williams. 2008."Compressed Air Energy Storage: Theory, Resources and Applications For Wind Power." Princeton Environmental Institute, Princeton University, April 8, 2008.

Sun, Y., L. Wang, G. Li, and J. Lin. 2010. "A Review of Analysis and Control of Small Signal Stability of Power Systems with Large Scale Integration of Wind Power." International Conference on Power System Technology (POWERCON), Hangzhou, China, October 24-28, 2010. Institute of Electrical and Electronics Engineers, Piscataway, NJ.

Swamy, D. 2011. "Development and Demonstration of a New-Generation, High-Efficiency, 10 kW Stationary Fuel Cell System." In DOE Hydrogen and Fuel Cells Program Annual Progress Report," DOE, Editor, Intelligent Energy, Long Beach, CA. pp. 894-898.

System Dynamic Performance Subcommittee, and Power System Engineering Committee. 1982. "Proposed Terms \& Definitions for Power Systems Stability." IEEE Transactions on Power Apparatus and Systems, vol. PAS-101, no. 7, July.

Tamura, Y., H. Mori, and S. Iwamoto. 1983. "Relationship between Voltage Instability and Multiple Load Flow Solutions in Electric Power Systems." IEEE Transactions on Power Apparatus and Systems, vol. PAS-102, pp.1115-1123, May 1983.

Tarasov, V.I. 1975. "Implementation of a Permanent Loading Procedure to Definition of Load Flows on a Limit of Aperiodic Steady-State Stability." Voprosy primeneniya matematicheskih metodov pri upravlenii regimami i razvitiem elektricheskih sistem, Irkutsk, pp. 50-56, 1975 - in Russian. 
Task Force on the Capacity Value of Wind Power, IEEE Power and Energy Society. 2011. "Capacity Value of Wind Power." IEEE Transactions on Power Systems, vol. 26, no. 2, May.

Taylor, C.W. 2008. "Voltage Stability for Undergraduates." University of Minnesota Power Group, Available. Accessed December 2, 2012.

Taylor P., et al. 1999. "A Summary of the State-Of-The-Art Of SMES, Flywheel, and CAES." Sandia Report SAND991854, July 1999. Sandia National Laboratories, Albuquerque, NM.

Thomas, M.H. 2003. "Persistence and Progress - The Zinc Bromine Battery in Renewable Energy Systems." In REGA FORUM 2003. Yarra Valley, Victoria, BC, Canada.

Tokuda, N. et al. 2000. "Development of a Redox Flow Battery System." SEI Technical Review, no. 50: 88-93.

Tong J, H.-D. Chiang, and T. P Conneen. 1993. "A Sensitivity-Based BCU Method for Fast Derivation of Stability Limits in Electric Power Systems." IEEE Transactions on Power Systems, vol. 8, no. 4, pp. 1418-1428, 1993.

Tsourakis, G., B. M. Nomikos, and C. D. Voumas. 2009A. "Effect of Wind Parks with Doubly Fed Asynchronous Generators on Small-Signal Stability." Electric Power Systems Research, vol. 79, no. 1, pp. 190-200, January.

Tsourakis, G., B. M. Nomikos, and C. D. Voumas. 2009B. "Contribution of Doubly Fed Wind Generators to Oscillation Damping." IEEE Transactions on Energy Conversion, vol. 24, no. 3, pp. 783-791, September.

Tsourakis, G., E. Potamianakis, and C. Vournas. 2004. "Eliminating Voltage Instability Problems in Wind Parks by Using Doubly Fed Induction Generators." In Proceedings of European Wind Energy Conference and Excibition, London, UK, 2004.

Tytgat, J. et al. 2011 "Umicore's Excellence in Battery Materials and Battery Recycling." Brussels, Available http://www.green-cars-initiative.eu/workshops/joint-ec-eposs-ertrac-expertworkshop-2011-on-battery-manufacturing/presentations/2 4\%20Jan\%20Tytgat Umicore.pdf. Accessed December 5, 2012.

Ullah, N.R., and T. Thiringer. 2008."Effect of Operational Modes of a Wind Farm on the Transient Stability of Nearby Generators and on Power Oscillations: A Nordic Grid Study." Wind Energy, vol. 11, no. 1, pp. 63-73.

Undervoltage Laod Shedding Task Force (UVLSTF), Technical Studies Subcommittee, Western Systems Coordinating Council. 1999. "Undervoltage Load Shedding Guidelines." July 1999. Available http://www.wecc.biz/committees/StandingCommittees/PCC/TSS/Shared\%20Documents/Underv oltage\%20Load\%20Shedding\%20Guidelines.pdf. Accessed November 28, 2012.

Union for the Coordination of Transmission of Electricity UCTE. 2003. Interim Report of the Investigation Committee on the 28 September 2003 Blackout in Italy. Press Release, Union for the Coordination of Transmission of Electricity UCTE, Brussels, Belgium, October 3, 2003. Available http://www.energie-schweiz.ch/imperia/md/content/medienmitteilungen $/ \mathrm{mm06}$ 122003/82.pdf. 
U.S. DOE. 2010. Solar Energy Technologies Program. Systems Integration Subprogram. Transmission Activity Draft, Multi-Year Plan (MYP) FY2010-FY2016, Energy Efficiency and Renewable Energy, U.S. Department of Energy, Washington, D.C. August 10, 2010

U.S. DOE. 1996. The Electric Power Outages in the Western United States July 2-3, 1996. DOE Report to the President, August 2, 1996. Available ftp://www.nerc.com/pub/sys/all updl/docs/pubs/doerept.pdf.

U.S. DOE. Undated. "U.S. Department of Energy." Washington, D.C. Available http://www.doe.gov. Accessed November 26, 2012.

Usaola, J. "Probabilistic Load Flow with Wind Production Uncertainty Using Cumulants and Cornish-Fisher Expansion." In Proceedings of the 2008 Power Systems Computation Conference (PSCC), Glasgow, UK, July 2008. Curran Associates, Red Hook, NY.

USE Supply, 2008, "20\% Wind Energy by 2030." December 2008. Available http://www.nrel.gov/docs/fy09osti/42864.pdf. Accessed November 29, 2012.

V-Fuel Pty Ltd, 2008 http://www.vfuel.com.au/infosheet.pdf. Accessed October 3, 2011.

Van Cutsem, T., I. Dobson (Editor), C. DeMarco, D. Hill, I. Hiskens, T. Overbye, M. Venkatasubramanian, and C. Vournas. 1998. Basic Theoretical Concepts, Chapter 2. IEEE Voltage Stability Work Group Report, April 1998. Institute of Electrical and Electronics Engineers, Piscataway, NJ. Available http://www.ece.wisc.edu/ dobson/WG/chapter.pdf. Accessed November 15, 2012.

Van Custem, T. 1991. "A Method to Compute Reactive Power Margins with Respect to Voltage Collapse." IEEE Transactions on Power Systems, vol. 6, no. 1, pp. 145-156, February 1991.

Van der Linden, S. 2006. "Bulk Energy Storage Potential in the USA, Current Developments and Future Prospects." Energy, vol. 31, no. 15, pp. 3446-3457.

Venikov, V.A., V. A. Stroev, V. I. Idelchick, and V. I. Tarasov. 1975. "Estimation of Electric Power System Steady-State Stability in Load Flow Calculation." IEEE Transactions on Power Apparatus and Systems, vol. PAS-94, pp.1034 - 1041, May/June, 1975.

Verhaeghe, F., F. Groubin, B. Yazicioglu, M. Schurmans, B. Thiis, G. Haesebroek, J. Tytgat, and M. Van Camp. 2011. "Valorisation of Battery Recycling Slags." Second International Slag Valorisation Symposium, Leuven, Belgium, April 4, 2011. Available http://www.slag-valorisationsymposium.eu/2011/images/papers/s4_12_verhaeghe.pdf. Accessed December 5, 2012.

Vittal, V., J. McCalley, V. Ajjarapu, and U. V. Shanbhag. 2009. Impact of Increased DFIG Wind Penetration on Power Systems and Markets. PSERC Publication 09-10, Power Systems Engineering Research Center, Tempe, AZ, October 2009. Available http://www.pserc.wisc.edu/documents/publications/reports/2009 reports/vittal dfig pserc final report s-34 2009.pdf. Accessed November 27, 2012.

Vivian, G.L., and G.T. Heydt. 1981. "Stochastic Energy Dispatch." IEEE Transactions on Power Apparatus and Systems, vol. PAS-100, no. 7, pp. 3221-3227, July. 
Vournas, C. 2004. Technical Summary on the Athens and Southern Greece Blackout of July 12, 2004. Available http://www.google.com/\#hl=en\&tbo=d\&output=search\&sclient=psy$a b \& q=T e c h n i c a l+S u m m a r y+o n+t h e+A t h e n s+a n d+S o u t h e r n+G r e e c e+B l a c k o u t+o f+J u l y+12 \% 2 C$ $+2004 \& o q=$ Technical+Summary+on+the+Athens+and+Southern+Greece+Blackout+of+July+12 \%2C+2004\&gs_l=hp.12...1108.1108.1.2142.1.1.0.0.0.0.122.122.0j1.1.0.les\%3B..0.0...1c.1.h3p2 3PeP2Fs\&psj=1\&bav=on.2,or.r_gc.r_pw.r_qf.\&bvm=bv.1355534169,d.dmQ\&fp=c341db92e3d4 $\mathrm{d} 65 \mathrm{~d} \& \mathrm{bpcl}=40096503 \& \mathrm{biw}=1600 \& \mathrm{bih}=78 \overline{5}$.

Vowles, D.J., C. Samarasinghe, M. J. Gibbard et al. 2008. "Effect of Wind Generation on SmallSignal Stability - A New Zealand Example." 2008 IEEE Power \& Energy Society General Meeting, pp. 5217-5224, 2008. Institute of Electrical and Electronics Engineers, Piscataway, NJ.

Walawalkar, R., J. Apt, and R. Mancini. 2006. "Economics of electric energy storage for energy arbitration and regulation in New York." Carnegie Mellon Electricity Industry Center Working Paper CEIC-06-04, Available https://wpweb2.tepper.cmu.edu/ceic/pdfs/CEIC 06 04.pdf. Accessed October 26, 2011.

Wan, H.B., and Y. H. Song. 1998. "Hybrid Supervised and Unsupervised Neural Network Approach to Voltage Stability Analysis." Electric Power System Research, vol. 47, no. 2, pp.115122.

Wang, X., H. Dai and R.J. Thomas. 1984. "Reliability Modeling of Large Wind Farms and Electric Utility Interface Systems." IEEE Transactions on Power Apparatus and Systems, vol. PAS-103, no. 3, pp. 569-575, March 1984.

Wang, X. L., et al. 2011 "Amorphous Hierarchical Porous GeOx as High-Capacity Anodes for Li Ion Batteries with Very Long Cycling Life." Journal of the American. Chemical Society, vol. 133, no. 51, pp. 20692-20695

Wang, J., X. Wang and Y. Wu. 2005. "Operating Reserve Model in the Power Market." IEEE Transactions on Power Systems, vol. 20, no. 1, February.

Weber, J. 2007. "Efficient Available Transfer Capability Analysis Using Linear Methods." PSERC Internet Seminar, November. Arizona State University, Tempe, AZ. Available www.powerworld.com/files/Efficient-ATC-Analysis.ppt

Wehenkel, L. 1998. Automatic Learning Techniques in Power Systems. Kluwer Academic Publishers, Boston, MA.

WECC. 2012. WECC Website. Available http://www.wecc.biz/About/Pages/default.aspx Accessed November 27, 2012.

WECC. 2010. Western Electricity Coordinating Council Off-Nominal Frequency Load Shedding Plan. December 2010. Available http://www.wecc.biz/library/Documentation\%20Categorization\%20Files/Policies/OffNominal\%20Frequency\%20Load\%20Shedding\%20Plan.pdf. Accessed November 9, 2012.

WECC. 2008. WECC System Performance Criteria. April 18, 2008, Western Electricity Coordinating Council, Washington, D.C. Available http://wecc.biz/standards/wecc\%20criteria/forms/allltems.aspx. Accessed November 15, 2012. 
WECC. 2007. WECC Standard BAL-STD-002-1 - Operating Reserves. March 12, 2007. WECC, Washington, D.C. Available http://www.nerc.com/files/BAL-STD-002-0.pdf. Accessed November 20, 2012.

WECC. 2006. Guide to WECC/NERC Planning Standards I.D: Voltage Support and Reactive Power. Reactive Reserve Working Group (RRWG), Western Electricity Coordinating Council, Washington, D.C. Available http://www.wecc.biz/committees/StandingCommittees/PCC/TSS/Shared\%20Documents/Voltag e\%20Stability\%20Guide.pdf. Accessed November 15, 2012.

WECC. 2005. Minimum Operating Reliability Criteria. Western Electricity Coordinating Council, Washington, D.C. April 2005. Available http://www.wecc.biz/library/WECC\%20Documents/Miscellaneous\%200perating\%20and\%20Pla nning\%20Policies\%20and\%20Procedures/WECC\%20Reliability\%20Criteria\%20MORC.pdf. Accessed November 15, 2012.

WECC. 1998. Voltage Stability Criteria, Undervoltage Load Shedding Strategy, and Reactive Power Reserve Monitoring Methodology. Final Report, Reactive Power Reserve Work Group, Western Electricity Coordinating Council, Washington, D.C. May 1998. Available http://www.wecc.biz/library/Documentation\%20Categorization\%20Files/Guidelines/Voltage\%20 Stability\%20Criteria\%20-\%20Guideline.pdf.

WECC, 2007, WECC Standard TOP-STD-007-0 - Operating Transfer Capability. Adopted by Board of Trustees, March 12, 2007. Available http://www.nerc.com/files/TOP-STD-007-0.pdf. Accessed December 5, 2012.

Western Governor's Association and U.S. Department of Energy. 2009. Western Renewable Energy Zones, Phase 1 Report. June. Available http://www.westgov.org/wga/publicat/WREZ09.pdf. November 9, 2012.

Western Systems Coordinating Council, Minimum Operating Reliability Criteria. Part III, WECC, Washington, D.C. Available http://www.westgov.org/wieb/meetings/crepc1000/briefing/morc.pdf. Accessed November 28, 2012.

Wets, R. J-B. 1996. "Challenges in Stochastic Programming." Mathematical Programming, vol, 75, pp. 115-135, 1996. Department of Mathematics, University of California, Davis. Available http://www.math.ucdavis.edu/ ribw/mypage/Stochastic Optimization files/Wets96 chall.pdf. Accessed December 5, 2012.

Whitney, J. 2011. "Ultra-High Voltage (UHV) Transmission is the Renewable Energy Interstate." The Green Economy Post, April 25, 2011. Available http://greeneconomypost.com/ultra-highvoltage-uhv-transmission-renewable-energy-interstate-14309.htm. Accessed November 20, 2012.

Wind. T.A. 2006. "Interconnection and Transmission for Wind Generation." Wind Utility Consulting, Jefferson, IA. Presented at WEATS 2006, Boulder, CO. Available http://apps1.eere.energy.gov/tribalenergy/pdfs/course wind wind3.pdf Accessed November 30, 2012. 
Winters, I.J. 2006. "Wind Out of their Sails." Mechanical Engineering, Available http://www.memagazine.org/contents/current/features/windout/windout.html Accessed November 27, 2012.

Wood, A. J, Wollenberg, B. L., "Power Generation Operation \& Control," 1984, John Wiley \& Sons, NY.

Wu, F. and S. Kumagai 1982. "Steady-State Security Regions of Power Systems." IEEE Transactions on Circuits and Systems, vol. 29, no. 11, November 1982.

Xiao, F., J. D. McCalley, Y. Ou, J. Adams, and S. Myers. 2006. "Contingency Probability Estimation Using Weather and Geographical Data for On-Line Security Assessment." In Proceedings of the 9th International Conference on Probabilistic Methods Applied to Power Systems, Institute of Electrical and Electronics Engineers,Stockholm, Sweden, June 11-15, 2006.

Xiao, J., Donghai Mei, Xiaolin Li, Wu Xu, Deyu Wang, Gordon L. Graff, Wendy D. Bennett, Zimin Nie, Laxmikant V. Saraf, Ilhan A. Aksay, Jun Liu, and Ji-Guang Zhang. 2011.

"Hierarchically Porous Graphene as a Lithium-Air Battery Electrode." Nano Letters (11):50715078.

Xu, X., M.W. Gustafson, B.P Lam, J.D. Mountford, and S.L. Johnson. 2002. "Assessment of Voltage Stability and Real and Reactive Margins Using Advanced Analytical Tools." In Proceedings of International Conference on Power System Technology PowerCon 2002, vol. 4, pp. 2047-2051. Institute of Electrical and Electronics Engineers, Piscataway, NJ.

Yang, Z. et. al. 2010. "Electrochemical Energy Storage for Green Grid." Chemical Reviews. vol. 111, no. 5, pp. 3577-3613.

Yang, Z., J. Zhang, M.C. Kintner-Meyer, X. Lu, D. Choi, J.P. Lemmon, and J. Liu. 2011.

"Electrochemical Energy Storage for Green Grid." Chem. Rev., vol. 111, no. 5, pp. 3577-3613

Yazicioglu, B., and J. Tytgat, 2011. "Life Cycle Assessments Involving Umicore's Battery Recycling Process," DG Environment Stakeholder Meeting, July 18, 2011.

Yee, H. and B. D. Spalding. 1977. "Transient Stability Analysis of Multimachine Power Systems by the Method of Hyperplanes." IEEE Transactions on Power Apparatus and Systems, vol. 96, no. 1 (Part 1), pp. 276-284, 1977.

Yip, T., C. An, G. Lloyd, M. Aten, and B.Ferris. 2009. "Field Experience of Dynamic Line Rating Protection for Wind Farm Connections." The International Conference on Electrical Engineering pp.2, Hong Kong, March 18-20, 2009.

Yu, H., C. Y. Chung, K. P. Wong, and J. H. Zhang 2009. "A Chance Constrained Transmission Network Expansion Planning Method With Consideration of Load and Wind Farm Uncertainties." IEEE Transactions on Power Systems, vol. 24, no. 3, pp. 1568-1576, August 2009

Yu, Y., Y. Zeng, C. Huang, S. T. Lee, and P. Zhang. 2004. "A Practical, Direct Method for Determining Dynamic Security Regions of Electrical Power Systems by Power Perturbation 
Analysis," International Conference on Electrical Engineering ICEE2004, July 4-8, 2004, Sapporo, Japan.

Zavadil, R. et. al. 2007, "Queuing up: Interconnecting Wind Generation into the Power System," IEEE Power \& Energy magazine, pp. 47-58.

Zeng, Y. and Y. Yu. 2002. "A Practical Direct Method for Determining Dynamic Security Regions of Electrical Power Systems." In Proceedings of International Conference on Power System Technology - PowerCon 2002, vol. 2, pp. 1270-1274, October 13-17, 2002.

Zeng, Y, Y. Yu, and H. Jia. 2005. "Theoretical Explanation of Hyper-Plane Boundary of Dynamic Security Region." In Proceedings of Canadian Conf. on Electrical and Computer Engineering, pp. 1946-1949, May 1-4, 2005.

Zhang, H. 2009. "R\&D Progress of Redox Flow Battery (RFB) for Energy Storage." MS\&T 2009 Conference \& Exhibition,

Zhang, J-G., Deyu Wang,Wu Xu, Jie Xiao, and R.E. Williford. 2010. "Ambient operation of Li/Air batteries." Journal of Power Sources, (195):4332-4337.

Zhang, P. and S. T. Lee. 2004. "Probabilistic Load Flow Computation Using the Method off Combined Cumulants and Gram-Charlier Expansion." IEEE Transactions on Power Systems, vol. 19, pp. 676-682, February.

Zhang, Y. et. al. 2009. "Progress of Electrochemical Capacitor Electrode Materials: A Review." International Journal of Hydrogen Energy, 34:p. 4889-4899.

Zhang, X.-P. 2004. "Multiterminal Voltage-Sourced Converter-Based HVDC Models for Power Flow Analysis." IEEE Transactions on Power Systems, vol. 19, no. 4, November, pp. 18771884.

Zhang, Y. et al 2010. "A Novel Bifunctional Electrocatalyst for Unitized Regenerative Fuel Cell." Journal of Power Sources, 195:p. 142-145

Zhang, Y., et al. 2009. "Progress of Electrochemical Capacitor Electrode Materials: A Review." International Journal on Hydrogen Energy, vol. 34, pp. 4889-4899.

Zhang, Y., et al. 2009. "Impacts of Solar PV Generators on Power System Stability and Voltage Performance." IEEE Power and Energy Society GM, San Diego, CA, pp. 1-7.

Zhao, P., H. Zhang, H. Zhou, J. Chen, S. Gao, and B. Yi. 2006. "Characteristics and Performance of 10kw Class All-Vanadium Redox-Flow Battery Stack." J. Power Sources, vol. 162, pp. 1416-1420. 